\title{
Development of a precision system for image-guided needle placement
}

- from scratch to clinic -

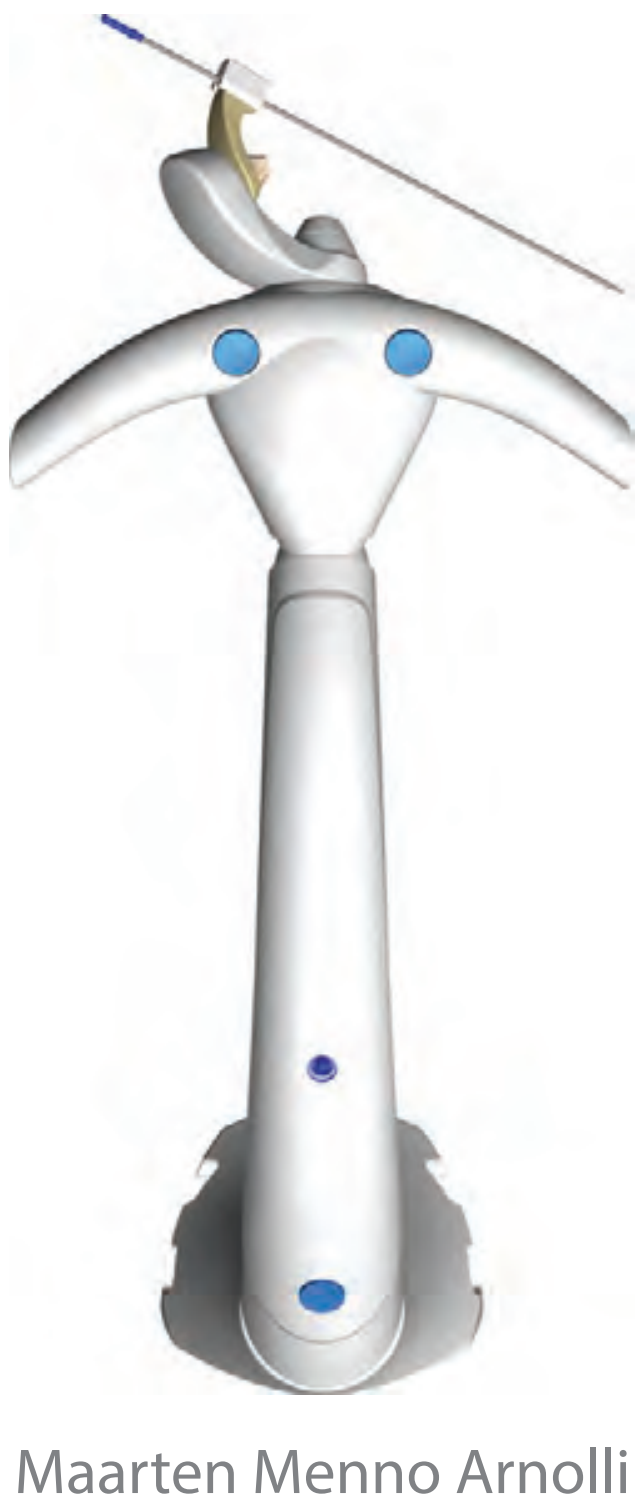




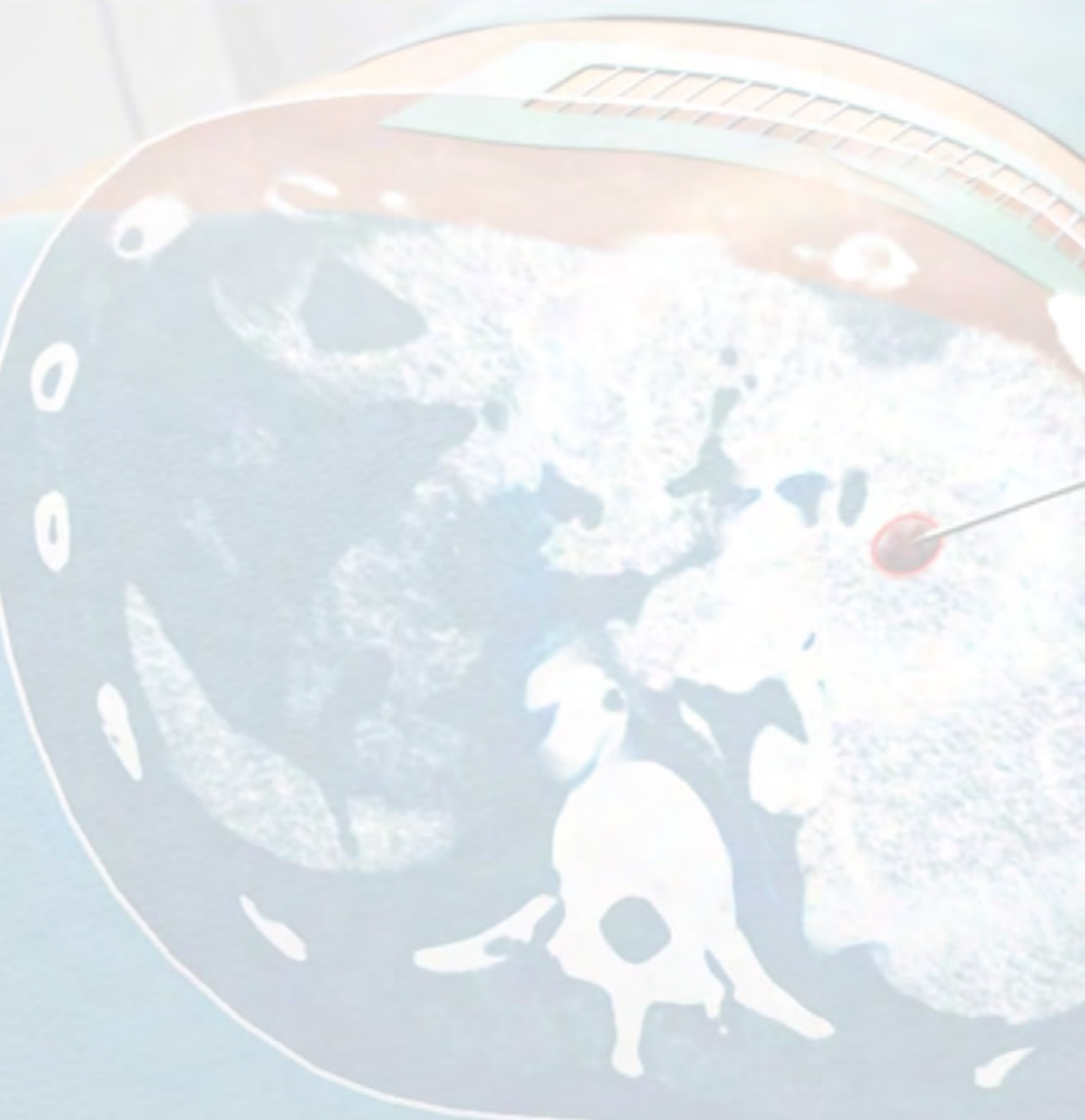




\section{Development of a precision system for image-guided needle placement}

- from scratch to clinic -

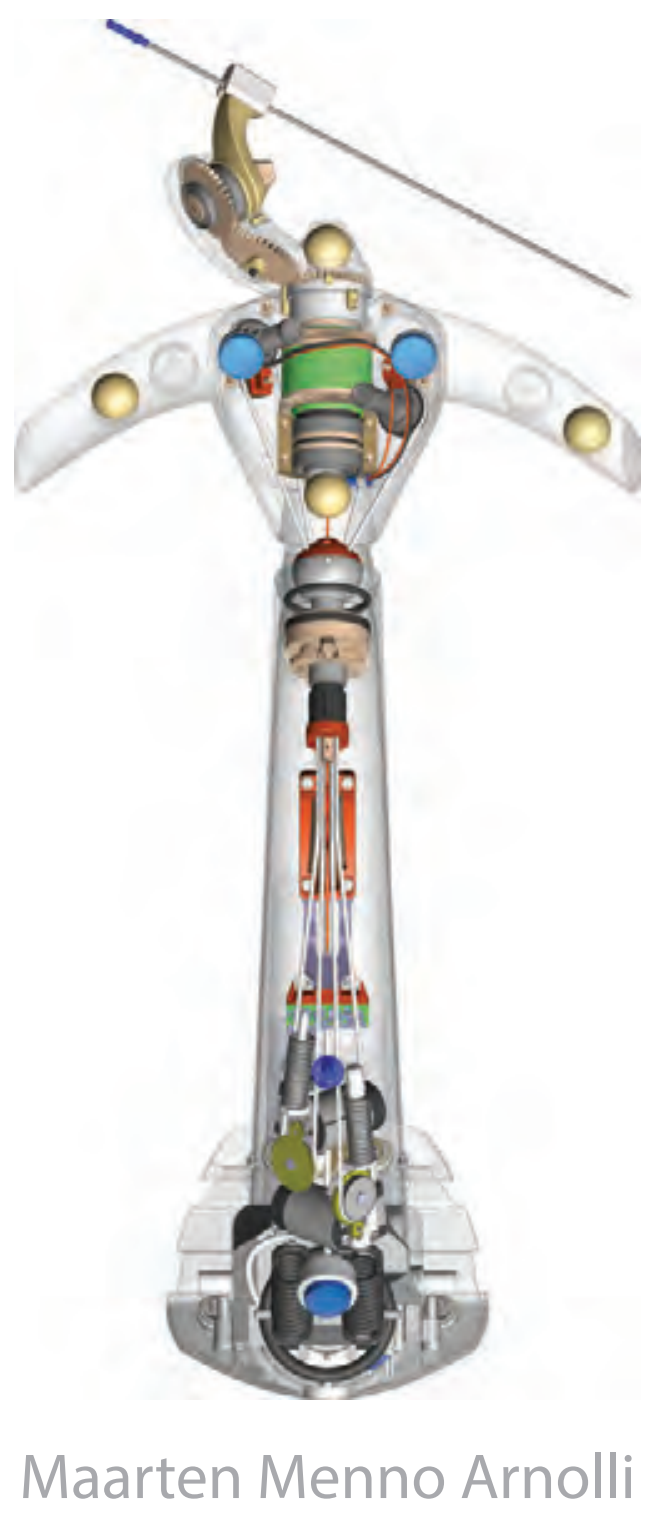


The research described in this dissertation has been conducted at DEMCON Advanced Mechatronics [1] in collaboration with the University of Twente [2], and has been funded by the following research programmes:

ITEA 2, 09039, Mediate - Patient-friendly medical intervention,

ITEA 3, 13031, Benefit - Better effectiveness and efficiency by measuring and modelling of interventional therapy,

SNN, Ricibion - Robotic interventions using CT-images for biopsies of lung nodules.

\section{DEMCON \\ UNIVERSITEIT TWENTE.}

Copyright (C) 2017 by Maarten Arnolli, the Netherlands.

All rights reserved. This book or any portion thereof may not be reproduced or used in any manner whatsoever without the express written permission of the copyright owner.

Printed by Ipskamp Printing, Enschede, the Netherlands.

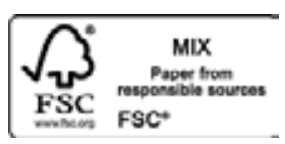

ISBN: $978-90-365-4332-3$

DOI: $\quad 10.3990 / 1.978903654332-3$ 


\title{
DEVELOPMENT OF A PRECISION SYSTEM FOR IMAGE-GUIDED NEEDLE PLACEMENT \\ - FROM SCRATCH TO CLINIC -
}

\section{DISSERTATION}

\author{
to obtain \\ the degree of doctor at the University of Twente, \\ on the authority of rector magnificus \\ prof.dr. T.T.M. Palstra, \\ on account of the decision of the graduation committee, \\ to be publicly defended
}

on Wednesday 24 May, 2017 at 14:45

by

\section{Maarten Menno Arnolli}

born on 13 July, 1983

at Noordlaren, the Netherlands 
This dissertation is approved by:

Prof.dr. I.A.M.J. Broeders (supervisor)

Prof.dr.ir. D.M. Brouwer (supervisor)

Dr.ir. M.C.J. Franken (co-supervisor) 


\section{Graduation committee}

Chairman \& Secretary: Prof.dr. P.M.G. Apers

Faculty of Electrical Engineering, Mathematics and Computer

Science, University of Twente, the Netherlands

Supervisors: Prof.dr. I.A.M.J. Broeders

Minimally Invasive Surgery \& Robotics, MIRA Institute for

Biomedical Technology \& Technical Medicine, University of

Twente, the Netherlands

Prof.dr.ir. D.M. Brouwer

Precision Engineering, Science-based Engineering, University of Twente, the Netherlands

Co-supervisor: Dr.ir. M.C.J. Franken

DEMCON Nymus3D, the Netherlands

Members: Prof.dr.ir. J.L. Herder

Mechanisms \& Robotics Design, CTIT Institute for ICT Research in Context, University of Twente, the Netherlands

\section{Prof.dr.ir. H. van der Kooij}

Biomechatronics \& Rehabilitation Technology, MIRA Institute for Biomedical Technology \& Technical Medicine, University of Twente, the Netherlands

Prof.dr. M.A.A.J. van den Bosch

Interventional Radiology, University Medical Center Utrecht, the Netherlands

\section{Prof.dr.ir. J. Dankelman}

Medical Instruments \& Bio-Inspired Technology, BioMechanical Engineering, Delft University of Technology, the Netherlands

Referees: Dr. K.P. de Jong

Hepato-Pancreato-Biliary Surgery \& Liver Transplantation, University Medical Center Groningen, the Netherlands

Dr. M.J.A. Jannink

Business Unit Medical Systems, DEMCON Advanced

Mechatronics, the Netherlands 
Paranymphs: Ing. M. Buijze

Mechanical \& Industrial Design Engineering, DEMCON Advanced Mechatronics, the Netherlands

Ing. M.Ph. Arnolli

Brother of the doctoral candidate 
To my parents, Ko \& Ria. 



\section{Summary}

Advances in medical technology enable an ongoing transition from invasive, open surgery to minimally invasive diagnostics and intervention. In the field of oncology, percutaneous techniques have emerged in which biopsy and ablation of tumours are performed via a needle through the skin using image-guidance. X-ray CT-guided needle placement in the thorax and abdomen is conventionally performed freehand. The performance of this method is user-dependent and multiple iterations are generally required to achieve satisfactory placement. Tissue damage, patient $X$-ray exposure, procedure time and costs increase with each placement iteration. The objective of this research is the development of a precision system for CT-guided percutaneous needle placement in the thorax and abdomen to improve upon the freehand method. It covers the entire strategic, architectural and detail design and realization of a complete system from first idea to prototype, its technical verification, its integration in the intervention suite and its pre-clinical validation, up to its first deployment in clinical practise.

The state of the art encompasses a wide variety of needle placement systems, from simple passive aids to fully automated robots. Despite the various attempted strategies, widespread clinical adoption of any system is lacking. A novel, patent pending system has been designed with a focus on clinical acceptability, applicability and usability. It provides a needle guide aimed by a 2-DOFs automated remote centre of motion (RCM) mechanism, connected to the CT table via a passive 6-DOFs linkage. The linkage enables a physician to manually place the RCM mechanism anywhere around the patient to coincide the RCM with the desired skin entry point and push-button lock it. The relevant anatomy of the patient and the system are CT scanned to acquire a 3D image, for automatic systemto-CT registration using incorporated fiducial markers, and for target specification and path approval in a graphical user interface. The needle guide is automatically aimed at the target, which is reached by a single manual needle insertion to indicated depth. Deceivingly simple in looks and usability, enabling the desired functionality and performance under requirements on X-ray transparency, size and shape, demanded resorting to unconventional solutions using ceramic and composite precision parts, remote actuation via endless Dyneema cables, fibre optical encoders, carbon nanotube electrical circuits and a pneumatic-hydraulic locking system, all customized and confined in a compact design.

In a comparative phantom study involving two experienced physicians, the performance of the system's guided method was proven superior to the freehand method in every aspect: successful needle placement was consistently achieved in a single insertion, with lower geometric placement errors, fewer CT scans, lower radiation dose and shorter placement time. The output of this research is a fully functional system, currently proving its merits in clinical practise. 


\section{Samenvatting}

Vooruitgang in medische technologie maakt een steeds verdergaande verschuiving mogelijk van invasieve, open chirurgie naar minimaal invasieve diagnostiek en interventie. Op het gebied van de oncologie hebben percutane technieken hun intrede gedaan, waarbij biopsie en ablatie van tumoren worden uitgevoerd via een naald door de huid met behulp van beeldgeleiding. Röntgen-CT-geleide naaldplaatsing in de thorax en abdomen wordt conventioneel handmatig uitgevoerd. De prestatie van deze methode is persoonsafhankelijk en meerdere iteraties zijn over het algemeen nodig om tot een toereikende plaatsing te komen. Met iedere iteratie nemen weefselschade, blootstelling van de patiënt aan röntgenstraling, en de doorlooptijd en kosten van de procedure toe. Het doel van dit onderzoek is de ontwikkeling van een precisie systeem voor CT-geleide percutane naaldplaatsing in de thorax en abdomen ter verbetering van de handmatige methode. Het beslaat het volledige strategisch, architectonisch en detail ontwerp en realisatie van een compleet systeem van eerste idee tot prototype, de technische verificatie, de integratie in de interventiekamer en de preklinische validatie, tot aan de eerste inzet in de klinische praktijk.

De stand van de techniek omvat een grote variëteit aan bestaande naaldplaatsingssystemen, van simpele passieve hulpmiddelen tot volledig geautomatiseerde robots. Ondanks de verscheidenheid aan strategieën blijft brede klinische adoptie van een systeem uit. Een nieuw systeem waarop octrooi is aangevraagd is ontworpen met een focus op klinische acceptatie, toepasbaarheid en gebruiksvriendelijkheid. Het biedt een naaldgeleiding die gericht wordt door een 2DOFs geautomatiseerd remote-centre-of-motion (RCM) mechanisme, gekoppeld aan de CT tafel via een passieve 6-DOFs arm. De arm stelt de arts in staat het RCM mechanisme overal rond de patiënt te plaatsen zodanig dat het RCM samenvalt met het gewenste entree punt op de huid, en het met één druk op de knop vast te zetten. De relevante anatomie van de patiënt en het systeem worden CT gescand om een 3D beeld te verkrijgen, voor automatische systeem-naar-CT registratie met behulp van ingebouwde referentiepunten, en voor specificatie van het doelwit en goedkeuring van het naaldpad in een grafische gebruikersomgeving. De naaldgeleiding wordt automatisch gericht op het doelwit, welke bereikt wordt in een enkele handmatige insertie tot de aangegeven diepte. Hoewel het uiterlijk en de bediening bedrieglijk simpel zijn, vereiste het mogelijk maken van de gewenste functionaliteit en prestatie onder de gestelde eisen aan röntgenstraling transparantie, grootte en vorm, een toevlucht tot onconventionele oplossingen, gebruikmakend van keramische en composiet precisiedelen, aandrijving op afstand via eindeloze Dyneema kabels, fiber-optische encoders, carbon-nanotube elektrische circuits en een pneumatisch-hydraulisch klemsysteem, allemaal op maat gemaakt en samengepakt in een compact ontwerp. 
In een vergelijkende fantoom studie met twee ervaren artsen is aangetoond dat de prestatie van de geleide methode van het systeem op ieder vlak superieur is aan de handmatige methode: succesvolle naaldplaatsing werd consistent in een enkele insertie bereikt, met lagere geometrische plaatsingsfouten, minder CT scans, lagere stralingsdosis en kortere plaatsingstijd. De uitkomst van dit onderzoek is een volledig functioneel systeem, dat op dit moment zijn meerwaarde aantoont in de klinische praktijk. 


\section{Contents}

Graduation committee

iii

Summary vii

Samenvatting $\quad$ ix

1 Introduction 1

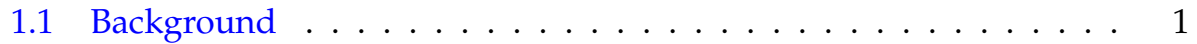

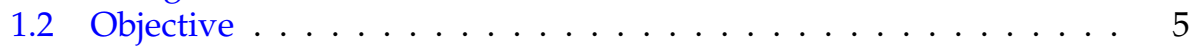

1.3 Contributions \& dissertation outline ................. 5

1.4 Scientific output . . . . . . . . . . . . . 7

2 An overview of systems for CT- and MRI-guided percutaneous needle placement in the thorax and abdomen 9

2.1 Introduction . . . . . . . . . . . . . . . . . 10

2.2 Materials \& methods . . . . . . . . . . . . . . . . . . 10

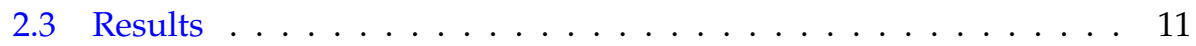

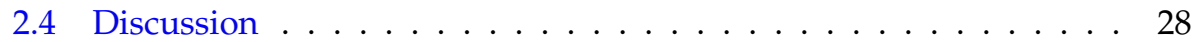

3 System for CT-guided needle placement in the thorax and abdomen - a design for clinical acceptability, applicability and usability 39

3.1 Introduction . . . . . . . . . . . . . . . . . . . 40

3.2 Materials \& methods . . . . . . . . . . . . . . . . . 41

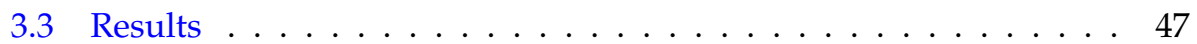

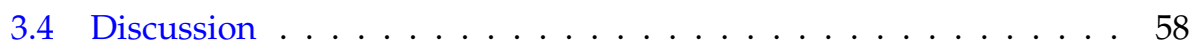

4 Deflection of needles for percutaneous procedures in the liver by axially guided insertion in ex vivo porcine liver tissue $\quad 61$

4.1 Introduction . . . . . . . . . . . . . . . . . 62

4.2 Material \& methods . . . . . . . . . . . . . . . . . 63

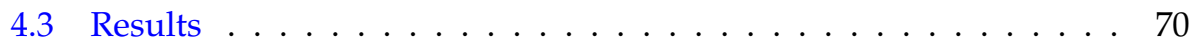

4.4 Discussion ........................... 72

5 A precision system for CT-guided needle placement in the thorax and abdomen - design \& performance analysis $\quad 75$

5.1 Introduction . . . . . . . . . . . . . . . 76

5.2 The registration system . . . . . . . . . . . . . . 78

5.3 The drive system . . . . . . . . . . . . . . . . . . . . 84

5.4 The structural link . . . . . . . . . . . . . . . . . 89 
5.5 The overall system $\ldots \ldots \ldots \ldots$

5.6 Discussion . . . . . . . . . . . . . . . . . . . 97

6 Validation of a system for CT-guided needle placement in the thorax and $\begin{array}{ll}\text { abdomen - a phantom study } & 101\end{array}$

6.1 Introduction . . . . . . . . . . . . . . . . . . . . . 102

6.2 Materials \& methods . . . . . . . . . . . . . . . . . . . . . 102

6.3 Results . . . . . . . . . . . . . . . . . . . . . 110

6.4 Discussion . . . . . . . . . . . . . . . . . . . . 115

7 Conclusions \& outlook 119

7.1 Conclusions . . . . . . . . . . . . . . . . . . . . . . . . 119

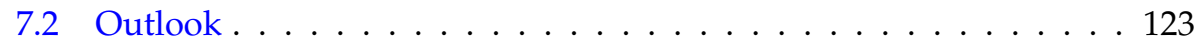

$\begin{array}{ll}\text { A Development history } & 131\end{array}$

$\begin{array}{ll}\text { References } & 169\end{array}$

$\begin{array}{ll}\text { Acknowledgements } & 191\end{array}$

$\begin{array}{ll}\text { About the author } & 201\end{array}$ 


\section{ChAPTER 1}

\section{Introduction}

\subsection{Background}

Advances in technology enable an ongoing transition from invasive, open surgery to minimally- or non-invasive diagnostics and intervention. This brings benefits such as less tissue damage, reduced pain, less scarring, lower risk of complications, shorter or no hospital stays and faster recovery. Established techniques find access to internal sites via natural orifices (e.g. colonoscopy and bronchoscopy), blood vessels (angioscopy) or minor incisions in the abdomen (laparoscopy) or thorax (thoracoscopy). In the field of oncology, image-guided percutaneous techniques have emerged, in which target tissue is approached through the skin by a needle.

\subsubsection{Percutaneous techniques in liver \& lung cancer}

Percutaneous techniques are frequently applied in the diagnostics and treatment of liver and lung cancer. Percutaneous biopsy, the analysis of a tissue sample harvested via a needle, has become a standard procedure in diagnostic pathology $[3,4]$. The gold standard for treatment with curative intent of liver tumours is surgical resection (hepatectomy) [5]. However, only 5 to $20 \%$ of patients are considered amenable to resection [6,7], because of limited hepatic reserve, a large number of tumours, a tumour's proximity to the main vascular or biliary structures and/or comorbidities. The gold standard for curative treatment of lung cancer is surgical resection of the involved lung lobe (pulmonary lobectomy), which is shifting in approach from invasive via an incision in the chest (thoracotomy) to minimally invasive surgery by thoracoscopy [8]. Percutaneous local ablation techniques provide a minimally invasive alternative for the treatment of tumours in the liver [9-12] and lungs [13-18]. Examples are radio frequency ablation (RFA) and microwave ablation (MWA): destruction of tissue by radio or microwave induced heat, ethanol ablation: destruction by alcohol insertion, cryoablation: destruction by freezing, and brachytherapy: destruction by ionizing radiation.

Epidemiological estimates from GLOBOCAN 2012 [19] emphasize the significance of the potential role for percutaneous techniques. Worldwide, 70 to $90 \%$ of primary liver cancer in adults is hepatocellular carcinoma (HCC) [20], caused by a viral infection with hepatitis B or C, or by cirrhosis, a disease which causes chronic liver damage. A worldwide incidence of 782.500 of primary liver cancer and a mortality of 745.500 has been estimated, with highest rates in East 


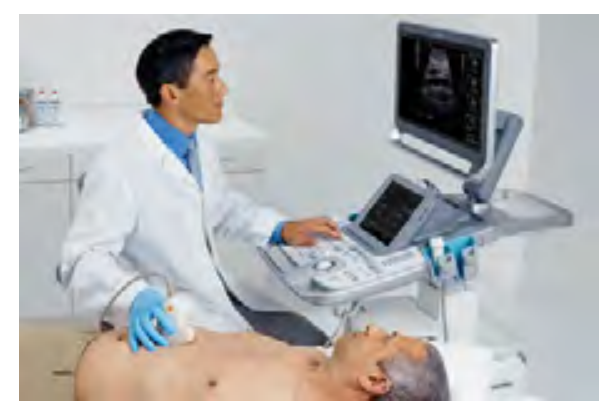

(a) Siemens [35] Acuson NX3 US system. (c) http://www.siemens.com/press.

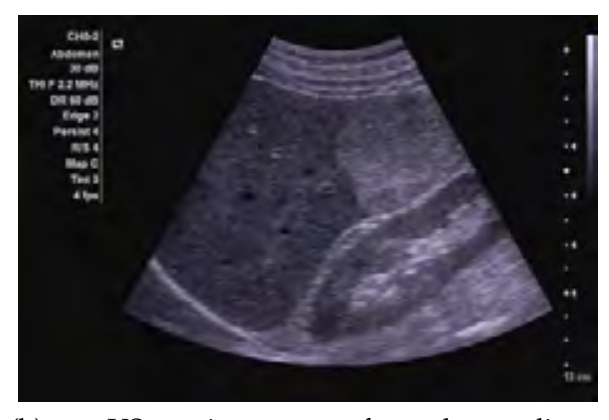

(b) US image of the liver. (C) http://www.siemens.com/press.

Figure 1.1: Ultrasonography.

and South-East Asia and Northern and Western Africa. Secondary liver cancer implies liver tumours are metastatic, originating in a different organ or part of the body. Colorectal liver metastases (CRLM) develop in 50\% of patients with colorectal cancer [21], which has an estimated worldwide incidence of 1.400 .000 and mortality of 693.900. Lung cancer has the highest estimated worldwide incidence and mortality, at 1.824 .700 and 1.589 .900 respectively.

\subsubsection{Image-guidance}

Percutaneous procedures rely on image-guidance for pre-procedural planning, needle targeting, treatment effect monitoring, intra-procedural treatment modification and post-procedural assessment of treatment response [22,23], provided by techniques such as ultrasonography (US), radiography, and magnetic resonance imaging (MRI).

US is based on tissue differences in echogenicity, the ability to bounce an echo. US is performed by decting echoes of ultrasonic waves emitted from a handheld transducer on the skin (Fig. 1.1). It is a readily available real-time imaging modality used for both percutaneous liver biopsy [24-26] and ablation [27-31]. Intravenous administration of micro-bubbles is widely used in Europe and Asia to provide sufficient contrast enhancement of blood vessels [32], but has only recently received approval for the liver in the USA [33]. US is unsuited for imaging of the lungs and the upper part of the liver behind the lung, because its field of view is blocked by air and bone [34]

Radiography is based on tissue differences in radiodensity, or transparency to X-ray. It includes classical two-dimensional projection imaging and computed tomography $(\mathrm{CT})$, in which cross-sectional and volumetric images can be reconstructed based on multiple one- or two-dimensional projections from different angles. X-rays are hazardous and exposure should be minimized. Spiral CT (ab- 


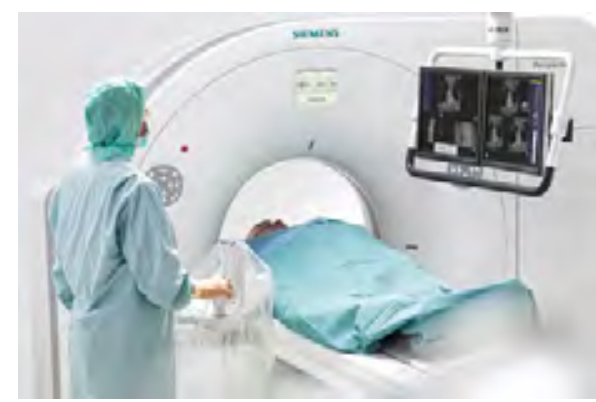

(a) Siemens [35] Somatom Perspective CT scanner. (C) http://www.siemens.com/press.

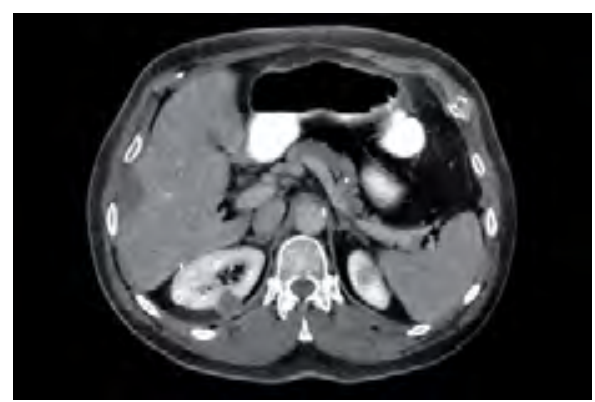

(b) CT image of a control study of a liver metastasis after microwave ablation treatment. (C) University Erlangen-Nuremberg, Erlangen, Germany.

Figure 1.2: Computed tomography.

breviated hereafter as $C T$ ) is implemented in conventional CT scanners (Fig. 1.2), in which a fan beam X-ray tube and one or more opposite detector rows rotate at up to $180 \mathrm{rpm}$ around a patient lying on a table. Spiral radiodensity data is acquired as the patient slides through the field of view, and reconstructed to a volumetric image array [36]. CT is widely used in both percutaneous liver biopsy [37,38], liver ablation [39,40], lung biopsy [4,41] and lung ablation [42-45]. $\mathrm{CT}$ is the imaging modality of choice for pre-procedural diagnostic and postprocedural assessment of treatment response (Fig. 1.2b). Whilst US is the more economical option for biopsy [46], CT provides images with higher resolution, superior tissue discrimination [47-49] and improved intra-procedural monitoring of thermal ablation zones [49]. CT fluorosopy provides real-time imaging of a single cross-section [50]. Cone beam CT (CBCT) uses a cone shaped X-ray beam and planar detector. This allows for both computed volumetric imaging in a single rotation around a patient and real-time projectional imaging, referred to as $C B C T$ fluoroscopy. Intended for interventional use, a C-arm configuration has been implemented to improve patient accessibility. CBCT is widely used for lung biopsy [51-54], and in lesser extent for tumour ablation in the liver [55-57] and lungs $[56,58]$. CBCT has poorer image quality and slower image reconstruction for volumetric imaging than CT [59], but projectional CBCT fluoroscopy may facilitate needle targeting and reduce procedure time $[58,60]$.

MRI is based on tissue differences in hydrogen concentration. The detection of hydrogen atoms exploits their behaviour to absorb and later re-emit electromagnetic radiation of a specific radio frequency related to the strength of an external magnetic field [61]. MRI (Fig. 1.3) provides superior soft tissue discrimination of organs such as the brain and prostate, and also the liver [47]. Clinical feasibility has been demonstrated of MRI-guided percutaneous biopsy and ablation in the 


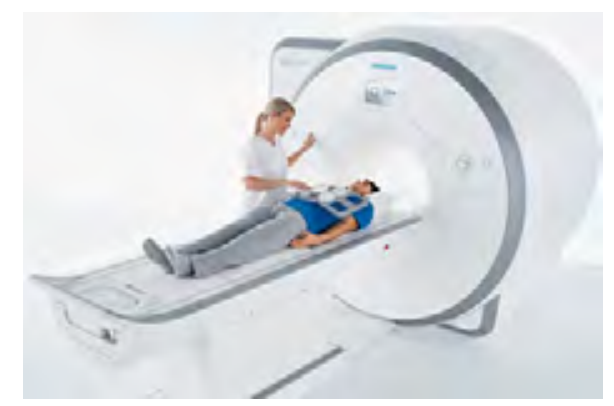

(a) Siemens [35] Magnetom Spectra MRI scanner. (C) http://www.siemens.com/press.

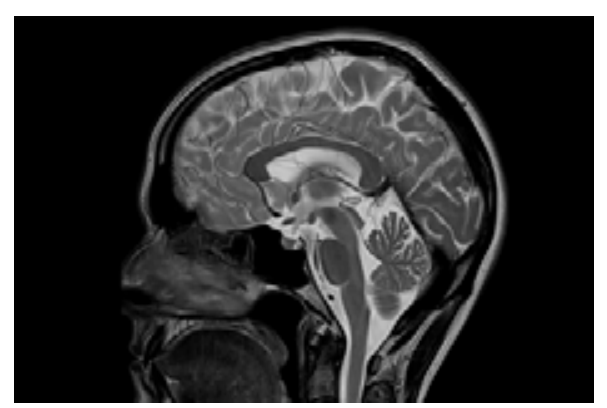

(b) MR image of the brain. (c) http://www.siemens.com/press.

Figure 1.3: Magnetic resonance imaging.

liver [62-67] and lungs $[63,68]$. These procedures are rare compared to US- and CT-guided due lower patient accessibility, long image acquisition time requiring a long patient breath hold, lower modality availability, the requirement on proximate medical devices to be compatible with high magnetic fields and radio frequency pulses, and associated costs $[47,69,70]$. Its predominant application remains high contrast pre- and post-procedural imaging.

\subsubsection{Opportunity}

Image-guided needle placement in the liver and lungs is an increasingly important clinical procedure due to the rise of percutaneous techniques. Whilst physicians have been equipped with high-tech imaging and therapeutic technology, needle placement is normally still performed freehand. The performance of this method is user-dependent and multiple iterations of needle placement and imaging for placement verification are generally required to achieve satisfactory placement. Particularly challenging are needle paths oblique to the transversal plane, which can be imperative to reach the cranial areas of the liver without puncturing the lung or to avoid impenetrable structures such as bones and critical structures such as larger blood vessels. Each placement iteration increases tissue damage with accompanied risks, patient and/or physician X-ray exposure in case of radiography, procedure time and costs. Precise needle placement according to path planning is an eminent opportunity for the multidisciplinary field of mechatronic system development to deliver added value and unlock the full potential of the available imaging and percutaneous technologies. 


\subsection{Objective}

The objective of the research described in this dissertation is formulated as:

the development of a precision system for image-guided percutaneous needle placement in the thorax and abdomen

CT is intended as the system's principal modality for image-guidance. Expansion to CBCT and MRI should be taken into account for their resemblance to $\mathrm{CT}$ in providing cross-sectional and volumetric images. The system should enable a lower number placement iterations, lower patient radiation exposure and a shorter placement time than the freehand method, and be designed with a focus on clinical acceptability, applicability and usability. The system should surpass the experimental and proof-of-principle stage and closely resemble a product ready for deployment in clinical practise. The research scope covers the entire strategic, architectural and detail design and realization of a complete system from idea to prototype, its technical verification, and its integration in the intervention suite and pre-clinical validation up to deployment in clinical practise.

\subsection{Contributions \& dissertation outline}

The primary output of the research described in this dissertation is the patent pending [71] system for CT-guided percutaneous needle placement shown in Fig. 1.4. The outline of this dissertation follows the chronological contributions to its development.

An overview has been created of the state of the art of systems for CTand MRI-guided percutaneous needle placement in the thorax and abdomen, to obtain an understanding of the different attempted strategies and their (dis)advantages. Systems found in scientific publications and patents are included, as well as commercially available products (chapter 2).

A problem analysis of the clinical worfklow and conventional freehand method of CT-guided needle placement has been performed. User requirements have been extracted and the strategy of a novel system for CT-guided percutaneous needle placement has been proposed, in which precise axial alignment of a needle guide is provided to reach a user-specified target in a single manual insertion. The architectural and detail design of the system have been elaborated, outlining its workflow and placement method. A first prototype has been realized and integrated in the intervention suite. The system's performance has been assessed for CT-guided needle placement in a static gelatin phantom (chapter 3). 


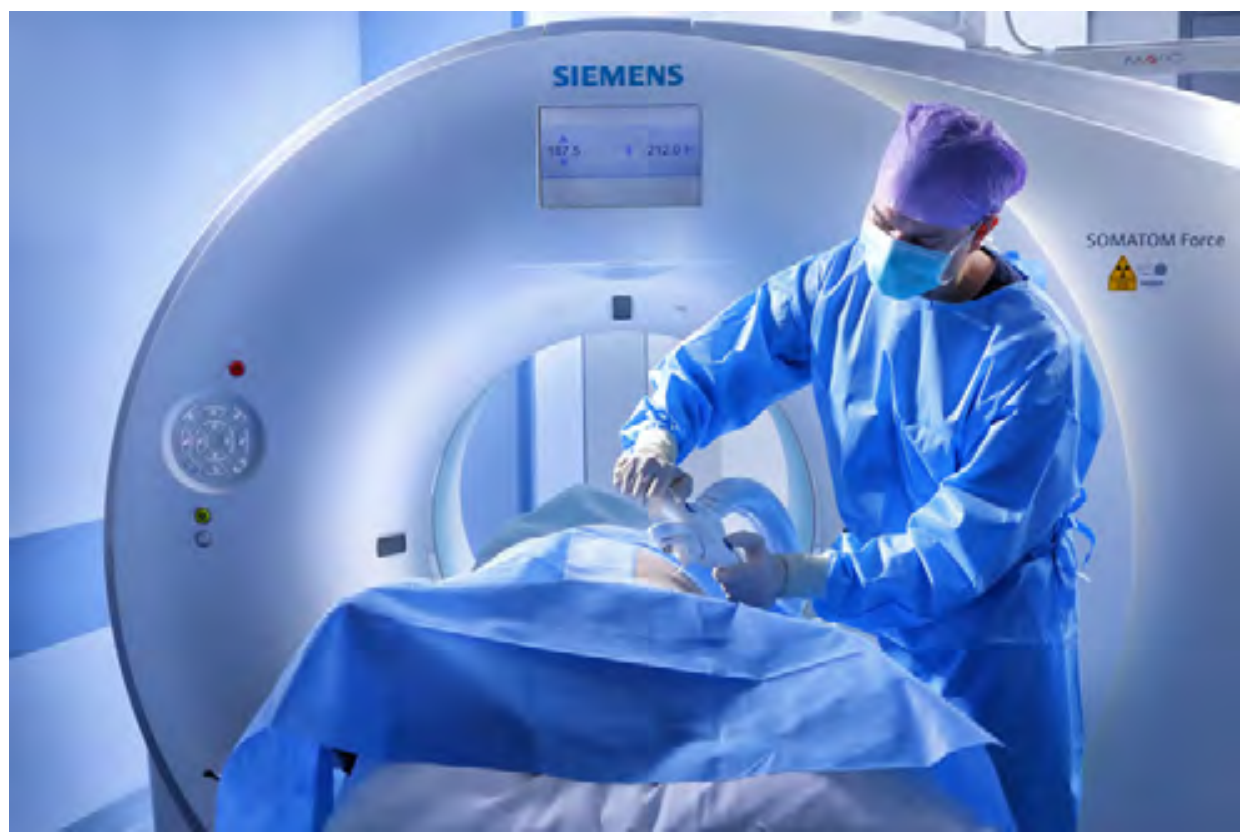

Figure 1.4: Preview of the developed system for CT-guided percutaneous needle placement.

In assessment of the system's strategy, the deflections of clinically used needles due to the needle-tissue interaction have been quantified for guided insertion in ex vivo porcine liver tissue (chapter 4).

The detail design of the novel system has been assessed from a technical point of view, quantifying the contribution of three prime subsystems to the needle placement error and that of the overall system (chapter 5).

A pre-clinical phantom study has been conducted to validate the performance of the novel system. The conventional freehand and the system's guided needle placement method have been employed by two experienced physicians to reach targets in a gelatin phantom and compared in terms of successfulness, number of needle manipulations, geometric placement errors, number of CT scans, radiation dose and placement time (chapter 6).

Chapter 7 presents the overall conclusions of this research and an outlook on developments and system applications derived from its prime objective.

Appendix A presents an illustrated overview of the development history of the novel system. 


\subsection{Scientific output}

The following publications have been delivered as output of this research, of which several form the body of this dissertation:

\section{Journal papers}

- M.M. Arnolli, N.C. Hanumara, M. Franken, D.M. Brouwer and I.A.M.J. Broeders. An overview of systems for CT- and MRI-guided percutaneous needle placement in the thorax and abdomen. Published in the International Journal of Medical Robotics and Computer Assisted Surgery, John Wiley \& Sons, Ltd., 11(4):458-475, 2015 [72].

- M.M. Arnolli, M. Buijze, M. Franken, K.P. de Jong, D.M. Brouwer and I.A.M.J. Broeders. System for CT-guided needle placement in the thorax and abdomen - a design for clinical acceptability, applicability and usability. Under review with the International Journal of Medical Robotics and Computer Assisted Surgery, John Wiley \& Sons, Ltd.

- M.M. Arnolli, A. Rosendaal, M. Franken, D.M. Brouwer and I.A.M.J. Broeders. Deflection of needles for percutaneous procedures in the liver during axially guided insertion in ex vivo porcine liver tissue. Submitted to the International Journal of Computer Assisted Radiology and Surgery, Springer.

- M.M. Arnolli, M. Buijze, M. Franken, I.A.M.J. Broeders and D.M. Brouwer. A precision system for CT-guided needle placement in the thorax and abdomen - design \& performance analysis. Submitted to the Journal of Medical Devices, American Society of Mechanical Engineers.

- M.M. Arnolli, W.J. Heerink, J. Pennings, D.M. Brouwer, I.A.M.J. Broeders and K.P. de Jong. Validation of a novel system for CT-guided needle placement - a phantom study. In preparation for submission to Radiology, Radiological Society of North America.

\section{Conference papers}

- M.M. Arnolli, M. Franken and D.M. Brouwer. CT registration: Experimental determination of suited fiducial marker material and registration errors. Journal of Medical Devices, Transactions of the ASME, 2013, 7(2), pages 020946-1-020946-2.

- M.M. Arnolli, K. Gunnink, D. Gelink, M. Buijze, M. Franken. A CTcompatible transmission for a needle placement system. Proceedings of the Dutch Society for Precision Engineering Conference 2014, September 2-3, 2014, Sint Michielsgestel, the Netherlands, page 41. 
- M.M. Arnolli, M. Buijze, M. Franken, K.P. de Jong, D.M. Brouwer and I.A.M.J Broeders. Development of a novel system for CT-guided percutaneous needle placement in the abdomen and thorax. Programme abstracts of the Design of Medical Devices Conference, Europe edition, October 22-24, 2014, Delft, the Netherlands, pages 90-93.

- M.M. Arnolli, W.J. Heerink, M. Buijze, M. Franken, M. Oudkerk, K.P. de Jong, D.M. Brouwer and I.A.M.J. Broeders. Development of a system for CT-guided needle placement: prototype realization \& preliminary performance assessment. Proceedings of the $28^{\text {th }}$ conference of the international Society for Medical Innovation and Technology / $4^{\text {th }}$ Design of Medical Devices Conference, Europe edition, October 5-8, 2016, Delft, the Netherlands.

\section{Patent application}

- M.M. Arnolli, M.C.J. Franken and M. Buijze, inventors. DEMCON Advanced Mechatronics B.V., applicant. System and method for aligning a medical device. World Intellectual Property Organization, WO2015/041516 A1, September 23, 2013.

\section{Public presentations \& demonstrations}

- M.M. Arnolli. CT registration: Experimental determination of suited fiducial marker material and registration errors. 2013 Design of Medical Devices Conference, April 8-11, 2013, Minneapolis, USA.

- M.M. Arnolli. A system for CT-guided needle placement for tissue biopsy and tumour ablation. Dutch Society for Precision Engineering Conference 2014, September 2-3, 2014, Sint Michielsgestel, the Netherlands.

- M.M. Arnolli. Development of a novel system for CT-guided percutaneous needle placement in the abdomen and thorax. Design of Medical Devices Conference, Europe edition, October 22-24 2014, Delft, the Netherlands.

- M.M. Arnolli. Development of a system for image-guided needle placement. Holland High Tech Roadmap Event 2015, September 22, 2015, 's-Hertogenbosch, the Netherlands.

- M.M. Arnolli. Development of a system for image-guided needle placement. LEO Robotics Congress 2016, April 21, 2016, Enschede, the Netherlands.

- M.M. Arnolli. A system for CT-guided percutaneous needle placement. $28^{\text {th }}$ conference of the international Society for Medical Innovation and Technology $/ 4^{\text {th }}$ Design of Medical Devices Conference, Europe edition, October 5-8, 2016, Delft, the Netherlands. 


\title{
CHAPTER 2
}

\section{An overview of systems for CT- and MRI-guided percutaneous needle placement in the thorax and abdomen}

\author{
Maarten M. Arnolli, Nevan C. Hanumara, Michel C.J. Franken, \\ Dannis M. Brouwer and Ivo A.M.J. Broeders \\ Published in the International Journal of Medical Robotics and Computer Assisted \\ Surgery, John Wiley E Sons, Ltd., 11(4):458-475, 2015 [72].
}

\begin{abstract}
Background Minimally invasive biopsies, drainages and therapies in the soft tissue organs of the thorax and abdomen are typically performed through a needle which is inserted percutaneously to reach the target area. The conventional workflow of needle placement employs an iterative freehand technique. This article provides an overview of needle placement systems developed to improve this method.
\end{abstract}

Methods An overview of systems for needle placement was assembled including those found in scientific publications and patents, as well as those that are commercially available. The systems are categorized by function and tabulated.

Results Over forty systems were identified, ranging from simple passive aids to fully actuated robots.

Conclusions The overview shows a wide variety of developed systems with growing complexity. However, given that only few systems have reached commercial availability, it is clear that the technical community is struggling to develop solutions that are clinically adopted. 


\subsection{Introduction}

Minimally invasive biopsies, drainages and therapies, such as microwave and radio frequency ablations, brachytherapy and cryotherapy, are typically performed through a needle which is inserted percutaneously to reach the target area, e.g. a tumour. Minimally invasive interventions offer benefits over traditional open surgery including less tissue damage, reduced pain, less scarring, lower risk of complications, shorter or no hospital stays and faster recovery. Selection of the target position, path planning and navigation of the needle are guided by imaging techniques such as computed tomography $(\mathrm{CT})$ or magnetic resonance imaging (MRI).

Placement of a needle according to plan is crucial for the success of both biopsies and therapies and many application specific systems have been developed to facilitate and improve needle placement. For example, in brain surgery which requires high accuracy and where the skull offers a fixed frame of reference, stereotactic and robotic systems for needle placement have been used clinically for decades [73,74]. Robotic solutions for ultrasound or MRI-guided needle placement into the prostate are another well-known class of needle placement systems $[75,76]$. An overview of general medical robotics including several needle placement systems has been presented by Taylor et al. [77] and a review of four interventional robotic needle placement systems was performed by Cleary et al. [78].

This article focuses on a specific group of systems: those that are intended to assist with CT- or MRI-guided percutaneous needle placement into the soft tissue organs in the thorax and abdomen, such as the liver, lungs and kidneys. A wealth of systems has been developed in the past decades, yet few have been clinically adopted and a freehand technique remains dominant. We provide an analysis of the conventional workflow of needle placement which employs an iterative freehand technique, followed by an overview of developed systems. We conclude with a discussion of trends in the art, leading to our perceptions regarding the most viable direction forward.

\subsection{Materials \& methods}

Multiple procedures of percutaneous needle placement in the abdomen at multiple medical centres were attended to analyse the conventional workflow. To assemble a comprehensive overview of existing systems for needle placement, systems presented in scientific publications and patents were searched for, as well as those that are commercially available. Various methods of organization were considered, including first date of publication and relative complexity, and selected was categorization by function. The systems were listed in a table, together with their commercial availability, successive geometric steps, corresponding number of degrees of freedom (DOFs), actuation type and reference or feedback sources. 
A qualitative comparison of the systems is avoided as not all test results of all systems are available and test methods differ per system.

\subsection{Results}

This section presents an analysis of the conventional workflow of freehand needle placement, followed by a categorized overview of needle placement systems.

\subsubsection{The workflow of freehand needle placement}

The analysis of the conventional workflow of needle placement presented in this section is based on clinical attendances at three medical centres, in the Netherlands and the USA. Fig. 2.1 shows a simplified flowchart of the workflow.

During a typical procedure, the patient is placed on the imager table and general anaesthesia may be applied. A first set of parallel, transversal images of the region of interest is acquired by scanning. The physician uses the image set to choose the target position for the needle tip as well as a suited entry point on the patient's skin, yielding the required insertion angles and depth. The needle path is chosen such that impenetrable structures such as bones and critical structures such as larger blood vessels are avoided, and preferably coincides with a transversal image plane to facilitate needle placement. For a needle trajectory oblique to the transversal plane, the gantry of some CT scanners can be tilted to visualize the entire needle trajectory in a single scan plane.

The next step is to transfer the planned needle path from the virtual 3D image space to the patient, starting with the entry point. The imager table is translated so that the transversal plane of the planned entry point coincides with the projected plane of a laser mounted at the top of the gantry, visualizing the intersection of this plane with the patient. The position of the entry point along this line is retrieved and marked by the physician based on anatomical references visible in the images, such as bones, or by using a sheet with radiopaque grid lines placed on the patient's skin during scanning. Sometimes a minor incision is made before proceeding to insertion to reduce tissue deformation, avoid abrupt needle entry through the skin and prevent entrainment of epithelial cells. If no general anaesthesia is applied, local anaesthetic may be injected at this point.

Now the physician must transfer the planned insertion angles from the virtual $3 \mathrm{D}$ image space to the patient and insert the needle accordingly. Longitudinal markings on the needle are used to measure the insertion depth. Depending on his or her confidence and the risk of damaging critical tissues, the physician may choose to insert the needle in a single stroke or, more typically, employ a stepwise procedure with intermediary confirmation scans and angular adjustments. Since therapeutic needles can be too long to fit within the geometric constraints of the imager bore when inserted only superficially, it is common practice to use a smaller aiming needle to iterate towards the right insertion angle before switching to 


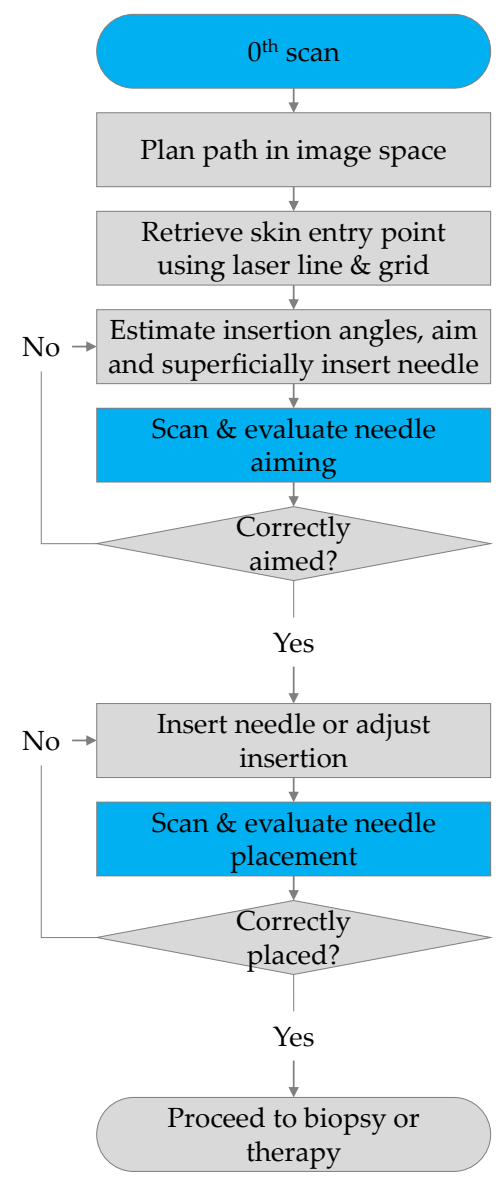

Figure 2.1: Simplified flowchart of the conventional workflow of freehand needle placement.

the therapeutic needle. This also avoids tissue deformation due to the gravitational forces acting on the usually heavier therapeutic needle. Once needle placement is completed, the physician may proceed to performing the biopsy, drainage or therapy.

Deviation of the target with respect to the planned needle path due to respiration is minimized by performing scanning and needle insertion during the same respiratory phase. This is accomplished by shortly pausing the mechanical ventilation in case of general anaesthesia, or asking the patient for a breath-hold in case of local anaesthesia. This 'respiratory gating' however does not completely compensate for organ motions or positional shifts.

The required number of iterations is highly dependent on individual physi- 
cian skill, particularly the ability to mentally process the 3D angular information in the image, transform it to the patient's reference frame, and then manually execute the desired angulation and insertion.

The iterative process of manual adjustments to needle placement and subsequent verification has three direct negative consequences: (1) increased tissue damage with each erroneous needle insertion, (2) increased radiation exposure of the patient and physician with each image scan in case of X-ray based imaging and (3) increased costs and equipment occupation due to increased procedure time. The smaller and deeper the target, while visible in imaging, the more challenging it is to target and herein lies the motivation to increase procedural speed and accuracy through the development of systems to aid in percutaneous needle placement.

\subsubsection{Needle placement systems}

What follows is the result of work to comprehensively identify and catalogue CT- and MRI-guided systems that intend to increase the quality of percutaneous needle placement in the thorax and abdomen. The systems range from simple passive aids to fully actuated, imager-integrated robots. They are divided into systems that supply feedback on placement of the needle to the physician and systems that supply physical guidance to the needle. The first group is further divided into navigation and tracking systems, systems that use the direction of gravity as a reference and systems that project a laser as an axis of reference. The latter group is divided based on mounting type: patient mounted, table mounted and gantry or floor mounted systems. The found systems are listed in Table 2.1.

\section{Systems that supply only feedback on needle placement}

In the current workflow, feedback on needle placement to the physician is supplied through the imaging modality, which in case of CT or MR imaging is typically non-real-time and does not allow for simultaneous needle placement adjustment. Therefore, one strategy to improve the procedure of percutaneous needle placement is to supply additional feedback based on other sources than the imaging modality.

Navigation \& tracking systems Navigation systems supply continuous realtime feedback to the physician on the position and orientation of a needle with respect to a patient's anatomy via a graphical representation of the needle in a static image set and/or a specified target coordinates. This requires a single registration of an image set and continuous registration or tracking of the instrument, both with respect to the same coordinate system. Three types of tracking methods are employed: optical, electromagnetic and mechanical. Fig. 2.2 illustrates the systems presented in this section. 


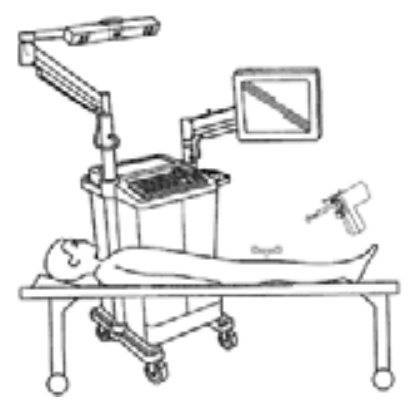

(a) Stryker [79] optical navigation system. Illustration adapted from [80].

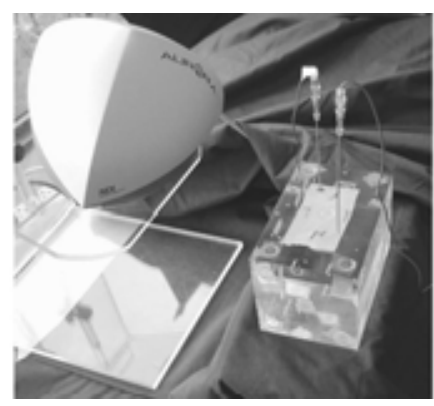

(c) NDI [83] Aurora. Illustration adapted from [84]. Copyright (C) 2007 by AUR. Reproduced by permission of AUR.

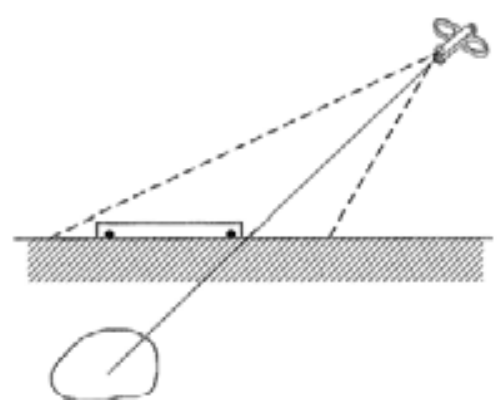

(b) ActiViews [81] CT-Guide. Illustration adapted from [82].

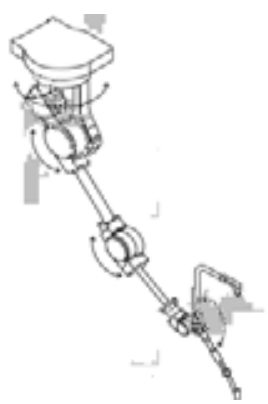

(d) Philips [85] Pinpoint. Illustration adapted from [86].

Figure 2.2: Navigation and tracking systems.

Optical tracking is the most commonly used technique in medical navigation systems. It incorporates infrared cameras to track light reflecting (passive) or emitting (active) markers attached to the instrument. Prior to imaging, several markers are commonly attached to the patient's skin, which are both visible in the images and captured by the tracking system to enable registration of an acquired image set with respect to the tracking system. Examples of optical tracking systems are the Claron [87] MicronTracker and the NDI [83] Polaris, which was used for navigation in phantom studies of CT-guided radiofrequency ablation in the liver in [88]. The NDI Polaris was also used in [89] to track both an instrument and a hand-held system which projects an image-overlay directly onto the tissue as an improved navigation method during open liver ablation. Several navigation systems which incorporate an optical tracking system are commercially available, e.g. the Stryker [79] Navigation System II and eNlite Navigation System, the Medtronic [90] StealthStation, the Pathfinder [91] Explorer and the CAScination [92] CAS-ONE. Optical tracking systems require an unobstructed 
line of sight between the cameras and the markers. This may hinder or restrict the workspace of the physician and equipment.

The ActiViews [81] CT-Guide [82] is an optical tracking system specifically designed for CT-guided needle placement. This system incorporates a compact disposable optical sensor which can be attached to the needle and a sterile sticker containing markers which can be identified in images from both CT and the optical sensor. The sticker is applied over the retrieved needle entry point on the patient's skin after planning of the needle path using a first set of CT images. A second set of CT images is obtained to perform a first one-time registration of the sticker with respect to the CT image set. The optical sensor is used to subsequently track the needle with respect to the sticker. Compared to conventional optical tracking systems, the ActiViews system is more compact, has a shorter line of sight between camera and markers which is less easily obstructed and integrates into the current workflow. Subsequent needle placement through a different entry point requires relocation of the sticker and repeated CT scanning.

Electromagnetic tracking systems consist of a generator of a controlled, changing magnetic field and an electromagnetic sensor containing one or multiple coils that is attached to the instrument. The position and orientation of the sensor and, thus, that of the instrument, with respect to the generator can be derived from the measured electromagnetic induction in the coils. Examples are the Medtronic [90] AxiEM and the NDI [83] Aurora. The latter system was used to track a needle with respect to a registered set of images in both phantom studies [84,93-95] and patient studies [96-98]. While electromagnetic tracking systems are unaffected by an obstructed line of sight, the presence of neighbouring metallic objects and other fields may seriously distort the magnetic field and increase registration errors.

Alternatively, a mechanical linkage with encoders on its joints can be used to track an instrument. This concept is used in the Philips [85] Pinpoint system $[86,99,100]$ which includes a needle guide at the tip of a passive, non-lockable Immersion [101] MicroScribe-G2X arm with five DOFs. Its base is fixed with respect to the imager gantry.

Systems that use the direction of gravity as a reference The direction of gravity can serve as a reference vector for the orientation of a planned axial trajectory. The imager table, the front face of the imager gantry or a laser line projected by the imager can serve as a second reference: rotation about the vector of gravity. With respect to these two reference vectors, a needle guide can be oriented such that it is parallel to the planned needle path, typically using a tool to indicate the relative angles. Maintaining this orientation, the needle guide can then be positioned to coincide with the entry point on the patient's skin, yielding full alignment with the planned needle path. Finally, insertion of the needle along the axial trajectory of the needle guide is easily measured using markings on the needle. This is the geometric method used in the systems presented in this paragraph, which are 


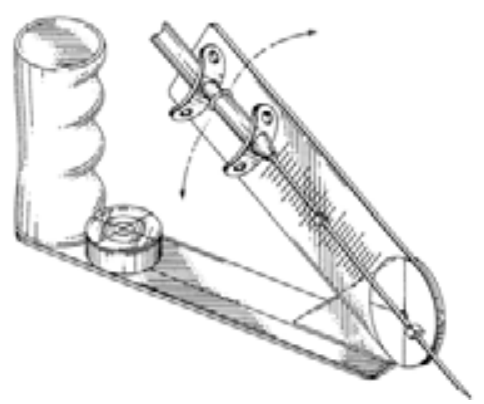

(a) Palestrant I. Illustration adapted from [102].

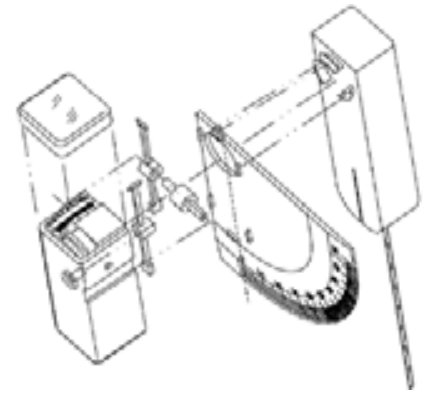

(c) Zhang et al. Illustration adapted from [104].

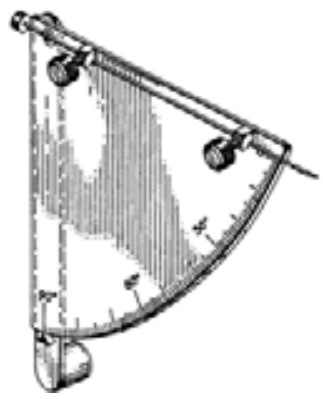

(b) Palestrant II. Illustration adapted from [103].

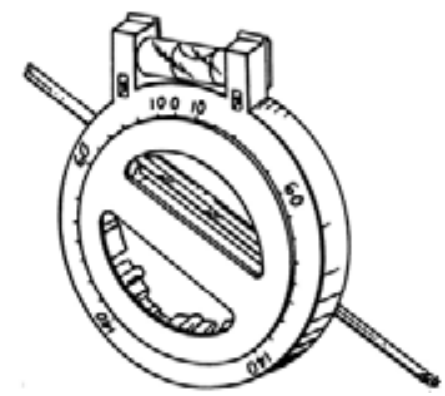

(d) INRAD [105] AccuPlace. Illustration adapted from [106].

Figure 2.3: Systems that use the direction of gravity as a reference.

illustrated in Fig. 2.3.

The use of the direction of gravity as a reference was applied in two needle guidance systems for CT-guided drainage and biopsy by Palestrant. The first system [102] embodies a two dimensional bubble level on a hand-held plateau with respect to which the angle of a needle guide about one axis can be set using a protractor, a common instrument to measure angles. The bubble level provides feedback on the orientation of the plateau for manual adjustment. A ruler is attached to the needle guide to determine the insertion depth. The system was shown to minimize trauma and decreased time for CT scanning in biopsy and drainage procedures in 40 patients [107]. The second system by Palestrant [103] embodies a needle guide attached to a hand-held protractor which can be manually rotated to the correct angle with respect to a pendulum. A two-dimensional version of the latter is patented by Zhang et al. [104].

The INRAD [105] AccuPlace [106] is a patented disposable commercially available system with the same functional concept. It is available in two versions, with a one and a two dimensional bubble level respectively. It relies on markings on the needle to determine the insertion depth. 
Systems that project a laser as an axis of reference Several systems project a laser along the planned needle trajectory, creating an axial reference in order to facilitate manual needle placement. First the tip of the needle is placed to coincide with the intersection of the laser with the skin, then the hub of the needle is aligned with the laser line and the needle inserted manually using markers on the needle to determine the insertion depth. Fig. 2.4 illustrates the laser projection systems presented in this section.

Frederick et al. developed a system in which the axial reference is formed by the intersection of two planes of light $[108,116]$. The system consists of two mechanisms fixed at the base with respect to the imager. Each mechanism contains protractors and rulers related to the coordinate system of the imager for manual adjustment of the placement of one plane of light. This system and the upcoming systems in this category use translation of the imager table in the direction of the gantry to position the patient with respect to the axial laser reference as part of its alignment with the planned needle path.

A small hand-held housing that projects a laser line as a reference for the needle path is presented by Ishizaka et al. [117]. The system needs to first be manually aligned with the front face of the gantry and then oriented to the correct insertion angle using a protractor and a pendulum indicator. Since the laser line is always kept parallel to this front face, the gantry must be tilted for laser guided needle placement oblique to the transversal plane.

A system by Unger et al. [109] comprises a laser module which can be coursely positioned around the patient by movement along two perpendicular horizontal rails. The module embodies several motion stages, mechanical constructions that allow motion in one or more directions whereas motion in other directions is constrained. Two perpendicular rotational stages and two perpendicular linear stages equipped with micrometers enable precise manual adjustment of the placement of the laser line in accordance with values determined by path planning using the images. A higher placement accuracy compared to freehand has been demonstrated in a phantom model [118].

The LAP [110] Patpos Invent $[119,120]$ is a variation of the system by Unger et al. The horizontal rail that holds the laser module is attached to the imager gantry above the patient. Like the system by Ishizaka et al. [117], gantry tilting is required for laser guided needle placement oblique to the transversal plane. A phantom study [111] showed improved accuracy with use of the system among inexperienced participants, whereas no significant improvement was found among experienced physicians. In a patient study [121], the system yielded a reduction in number of scans, number of needle placement corrections, targeting error and procedure duration. An extension of the system adds a vertical rail between the horizontal rail and the laser module to increase the range of the system. A similar system registered with the CT imager and with automated translation and rotation of the laser was patented by Siemens [35,122].

The commercially available NeoRad [112] SimpliCT [113,123,124] is very similar to the system by Unger et al. [109], but streamlined. This system embodies a 


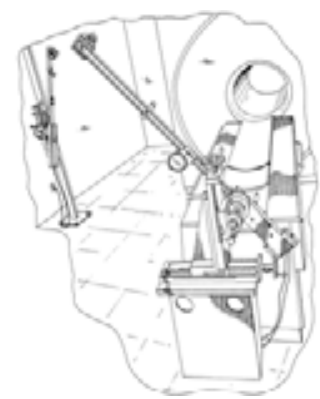

(a) Frederick et al. Illustration adapted from [108].

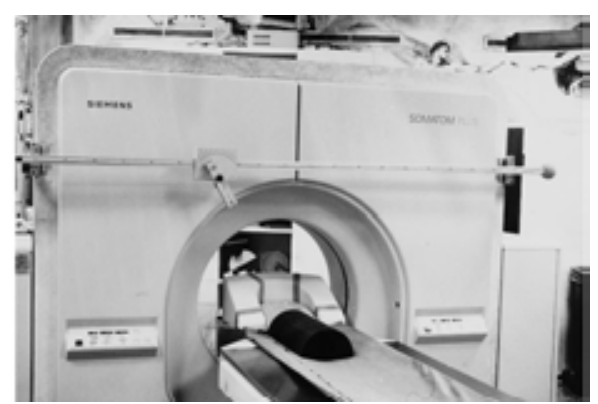

(c) LAP [110] Patpos Invent. Illustration from [111]. Copyright (C) 1999 by Springer-Verlag Berlin Heidelberg. Reproduced by permission of Springer-Verlag Berlin Heidelberg.

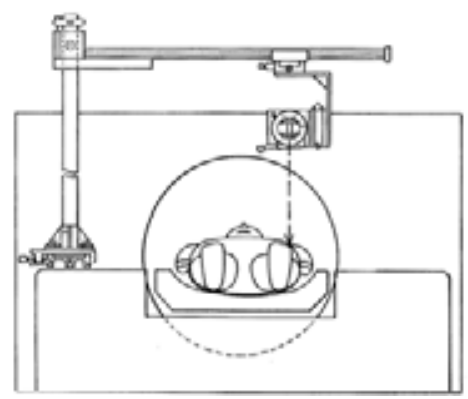

(b) Unger et al. Illustration adapted from [109].

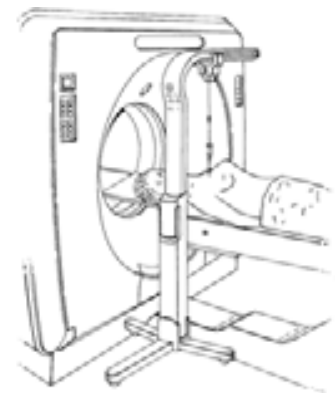

(d) NeoRad [112] SimpliCT. Illustration adapted from [113].

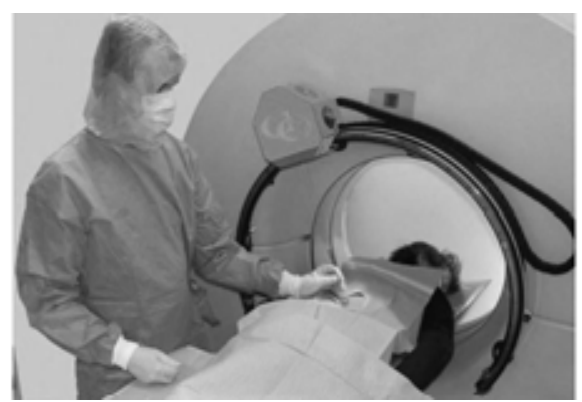

(e) Amedo [114] LNS. Illustration adapted from [115]. Copyright (C) 2013 by Elsevier Inc. Reproduced by permission of Elsevier Inc.

Figure 2.4: Systems that project a laser as an axis of reference. 
housing hanging horizontally on a floor mounted frame or a ceiling mounted arm and aligned with the imager table. A laser line is projected from the housing. The two lateral angles of this line can be adjusted through a user interface according to the values obtained from path planning. The last step before needle insertion is to manually translate the housing to let the laser line intersect with the retrieved entry point. The system received positive clinical feedback by Krombach et al. [125] who used the system for nine percutaneous CT-guided nerve blocks in eight patients. Successful guidance of a needle by the system into two kidney tumours of a single patient was reported by Varro et al. [126]. In a further version of the system, the housing is ceiling mounted via a moveable arm, which makes its positioning easier, allows for a larger range of needle paths and obstructs less workspace.

The commercially available Amedo [114] LNS [127] is similar to the LAP Patpos Invent, but incorporates an arc-shaped rather than linear guidance for a laser module concentric with the imager bore to accommodate for a larger range of insertion angles. The position and 1-DOF rotation of the laser module are automatically set to align the laser line with the planned needle path. The system has been validated by a randomized controlled clinical trial of CT-guided spinal interventions in comparison to the conventional workflow [115], showing an increase in procedure precision and speed, and reduction of radiation exposure.

Philips [85] used a 6-DOFs Kawasaki FS2 articulated arm, to place a laser reference for the needle trajectory. The arm was mounted on a CT scanner gantry and registered with its coordinate system [128, 129], for integration of imaging and needle placement. This is the only system in this category which does not need translation of the imager table to fully align the axial laser reference with the planned needle path, though translation of the imager table is still required for initial coarse positioning of the patient within its range of motion.

\section{Systems that supply physical guidance}

The systems described in the previous section rely on the physician to manipulate the needle. This section will discuss systems which apply physical guidance to the needle, in order to potentially reduce placement errors and user-dependency. These systems are further divided based on their mounting type: patient mounted and table, gantry or floor mounted.

Patient mounted systems Placing a physical guidance system directly upon the patient may reduce needle placement errors due to tissue movement, assuming that the movement of a target is coherent with that of the patient's skin. Whether this assumption is valid depends on the depth of the target as well as the body site. Patient mounted systems typically enter the imaging field with the patient, which yields system requirements regarding imager compatibility, relating to system size and materials. In addition, low weight is desired to avoid patient 


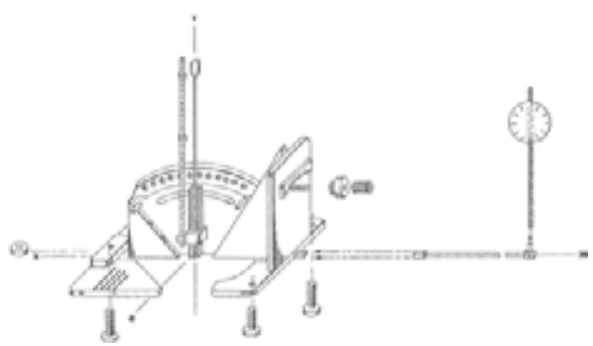

(a) Chang et al. Illustration adapted from [130].

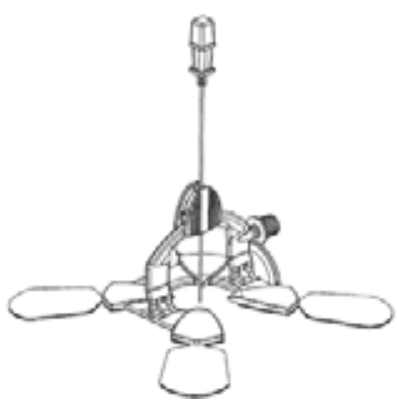

(b) Neorad [112] Simplify. Illustration adapted from [131].

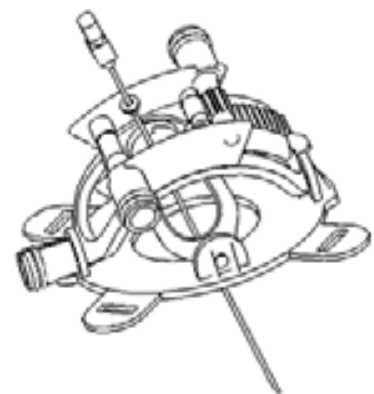

(d) Robopsy. Illustration adapted from [134]. (c) Apriomed [132] SeeStar. Illustration adapted from [133].

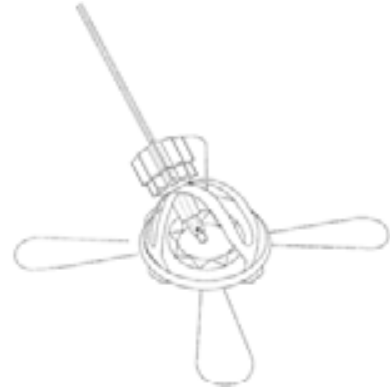

Figure 2.5: Patient mounted systems that supply physical guidance.

discomfort. Fig. 2.5 illustrates the patient mounted physical guidance systems presented in this section.

A patent by Chang et al. [130] describes one of the simplest patient mounted needle guidance systems. It embodies a flat adhesive plateau which can be attached to the patient's skin centred around the retrieved entry point. On top of the plateau is a mechanism which allows for a needle guide to be manually rotated about two orthogonal axes intersecting with the entry point. The needle guide can be locked once a correct orientation has been achieved. The Neorad [112] Simplify [131] and Apriomed [132] Seestar [138] are comparable commercially available systems.

The Neorad [112] Simplify [131] system consists of a pivoting arc that allows rotation of the needle guide. The system does not directly help to aim the needle, but merely holds a needle in place to allow for imaging before or during needle insertion without the need for a physician to manually hold the needle, avoiding radiation exposure to the physician.

The same benefits are offered by the Apriomed [132] Seestar [133, 138], whose 


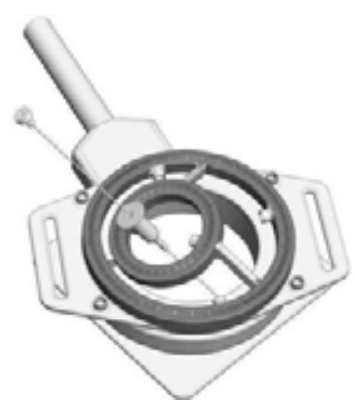

(e) Song et al. Illustration adapted from [135]. Copyright (c) 2013 by IEEE. Reproduced by permission of IEEE.

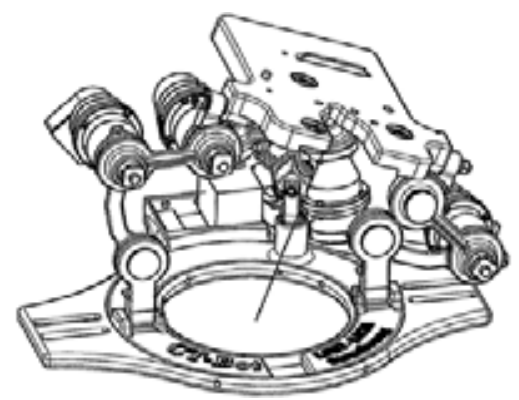

(f) CT-Bot. Illustration adapted from [136].

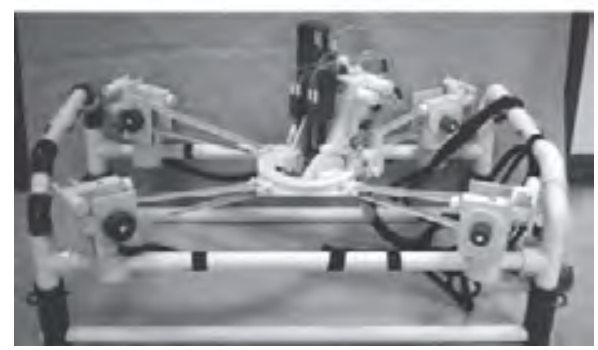

(g) LPR. Illustration from [137]. Copyright (C) 2008 by IEEE. Reproduced by permission of IEEE.

Figure 2.5: Patient mounted systems that supply physical guidance - continued.

mechanism consists of two perpendicular pivoting arcs over which a needle guide can be moved and locked. In addition, the system incorporates a metal tube which yields deliberate streaking in CT images to indicate the current axis of guidance. In a patent describing the Seestar [139], a variation of the system is presented consisting of a needle guide on a partial sphere which can rotate on a half dome and can be locked. In contrast to the system by Chang and the Simplify system, the Seestar cannot be removed after needle insertion without retraction of the needle, which is a limitation.

The Robopsy system [134, 140] for CT-guided percutaneous biopsies has a kinematic construction which is identical to that of the Apriomed Seestar and is equipped with two electrical stepper motors for active needle aiming. Two additional stepper motors allow for automated clamping and releasing of a needle and its insertion. This allows a needle to move with organ motion after insertion and removal of the system without retraction of the needle. The system can 
be placed such that the four motors are located outside the transversal planes around the needle trajectory to avoid image distortion. Means for registration were not included yet, but course manual alignment of the system with the imager's axes facilitates remote manual control of the needle with feedback from CT images through a graphical user interface. Targeting of a lesion during an in vivo porcine trial was successfully accomplished using a total of four image scans [141]. Wu et al. subsequently developed a spherical needle manipulator mounted on an MRI loop coil [142]. Piezo stepper motors automate its two DOFs and registration with the imager is performed through six spherical fiducials.

Yet another 2-DOFs manipulator fitted onto an MRI loop coil was developed by Song et al. [135]. It contains a needle guide which can be oriented about two axes through manual rotation of two serially stacked rings. After registration of the system with the MRI scanner, the required rotation angles of the rings corresponding to the planned needle path can be calculated automatically and manually set using protractors.

The CT-Bot $[136,143,144]$ comprises a CT-guided, 5-DOFs parallel mechanism actuated using ultrasonic piezo motors. It uses a fiducial configuration for registration of the robot with the imager. The mechanism aligns a 2-DOFs needle driver which automates insertion and spinning of the needle. Haptic control over needle insertion is incorporated.

The CT and MRI compatible Light Puncture Robot (LPR) [137,145] embodies a patient mounted active needle insertion stage which can be oriented about two axes. Four actuators on a frame fixed to the imager table are connected to the stage via straps to enable automated movement of the stage over the patients abdomen. Registration of the system with a CT imager is performed using a fiducial configuration. The actuators of the system are custom pneumatic stepper motors. A further version of the system is under development [146]. Both the LPR and the CT-Bot [143,144] occupy a significant part of the imager bore, leaving little space for the patient.

Table, gantry \& floor mounted systems Table, gantry and floor mounted systems that provide physical guidance help placing a needle along a linear trajectory fixed with respect to the imager gantry or table coordinate system rather than the patient's skin. Table mounted systems, like patient mounted systems, typically enter the imaging field, yielding system requirements on imager compatibility. Fig. 2.6 and Fig. 2.7 illustrate the table, gantry and floor mounted systems presented in this section.

Bard [147] developed the CT Guide [148, 156], a table mounted stereotactic frame for needle placement. It consists of a base which is fixated between the table and the patient, a linear slide parallel to the table, an arc-shaped arm which can pivot about a first axis with respect to the slider, and a needle guide which can be positioned along the slider and pivot about a second axis. The angle of the arc-shaped arm and the needle guide can be determined by protractors. The 


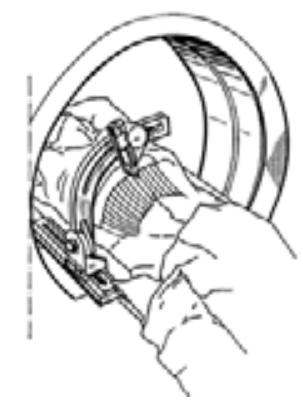

(a) Bard [147] CT Guide. Illustration adapted from [148].

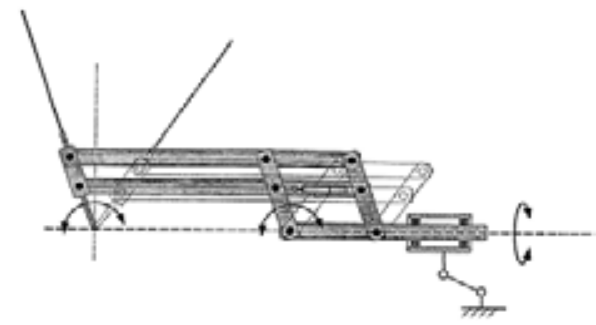

(c) Siemens [35]. Illustration adapted from [150].

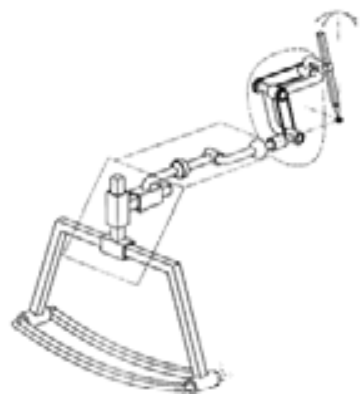

(e) AcuBot. Illustration adapted from [153].

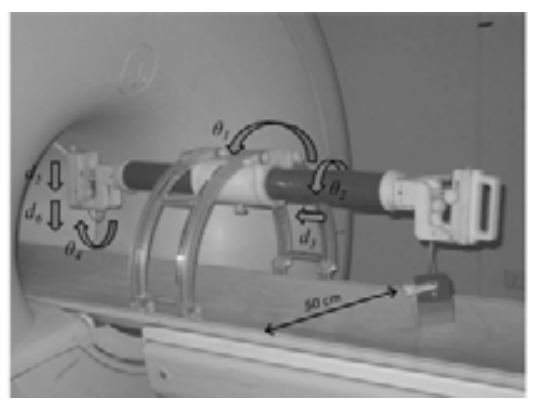

(b) Christoforou et al. Illustration adapted from [149]. Copyright (c) 2013 by John Wiley \& Sons, Ltd. Reproduced by permission of John Wiley \& Sons, Ltd.

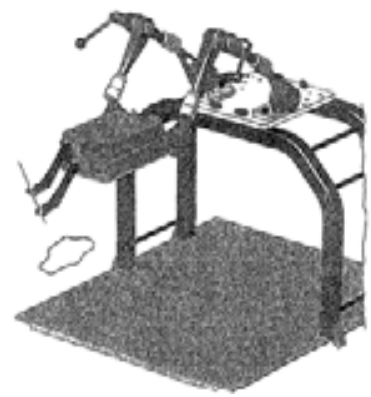

(d) iSYS Medizintechnik [151] iSYS1. Illustration adapted from [152].

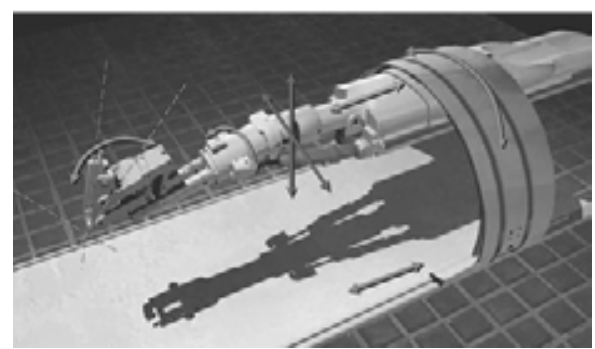

(f) INNOMEDIC [154] INNOMOTION. Illustration adapted from [155]. Copyright (C) 2008 by IEEE. Reproduced by permission of IEEE.

Figure 2.6: Table mounted systems that supply physical guidance. 
needle guide includes an engagement mechanism and a ruler to determine the insertion depth. The system could not be used in 32\% of 107 patients involved in a clinical test [157], primarily due to the constraints posed by the system on patient size or the need for a lateral needle path. When the system was used it decreased the number of iterations needed to correctly place the needle.

To avoid issues related to MRI compatibility, manual actuation was chosen for a manipulator developed by Christoforou et al. [149]. The system is designed to fit in between patient and imager bore and contains five DOFs to place a needle guide. Two thin tubes filled with gadolinium contrast agent are attached to its end effector parallel to the needle to indicate its placement in the images which are used for feedback on its placement. A sixth DOF for needle insertion is connected via cables to a crank on a hand-held unit.

A system by Siemens $[35,150,158]$ includes a needle guide on an active 2-DOFs remote centre of motion mechanism, consisting of a pivoting parallelogram. The mechanism can be placed and held by an arm such that the remote centre of motion coincides with the retrieved entry point.

The iSYS Medizintechnik [151] iSYS1 [152] evolved from the Biopsy Robot I and II systems. The system embodies a needle guide which is connected in parallel to two stacked actuated XY stages, which enable 2-DOFs lateral positioning and 2-DOFs lateral orienting. A mechanical arm is used to lock the system after coarse manual placement near the site of the intervention. Placement of the needle guide can then remotely controlled under live imaging without X-ray exposure to the physician. Included is a fiducial marker configuration consisting of two parallel rings concentric with the needle guide. Using live image guidance of a C-arm cone beam CT, which can automatically align its imaging direction with the planned needle path, the needle guide can be appropriately placed in two steps. First the top XY stage is moved until both fiducial rings appear concentric in the image, indicating the axis of the needle guide is parallel to the planned needle path. Then the lower XY stage is moved to perform a lateral translation of the needle guide until both fiducial rings appear concentric in the image with the planned needle path, implying the axis of the needle guide is coincident with the planned needle path. Subsequent needle insertion through the needle guide is performed manually.

The AcuBot $[153,159]$ is a 5-DOFs robotic needle placement system placed on a bridge frame over the patient. The system consists of a 3-DOFs linear XYZ stage to position a 2-DOFs remote centre of motion mechanism with a needle guide. In addition, a subsystem for automated gripping, insertion and axial rotation of the needle has been developed [160]. A fiducial configuration of seven aluminium rods is connected to the system's end effector. The rods form three Z-shaped motifs which enable registration with the imager through a single image scan in a wide range of rotations [161].

The Innomedic [154] INNOMOTION [155,162] is a CT and MRI compatible robotic system for needle placement. The system incorporates a 2-DOFs remote centre of motion mechanism identical to the one used by Siemens $[35,150,158]$. 
The 2-DOFs remote centre of motion mechanism and its 3-DOFs placement are actuated by linear pneumatic cylinders and feedback controlled using fibre-optic encoders. Spherical markers filled with gadolinium contrast agent are attached to the end effector for direct registration of the system with an MRI scanner. Coarse placement of the system over the patient to reach the site of the intervention is performed manually. An additional actuated needle insertion module remained in experimental stage. After validation of the system's targeting precision and tests in porcine models, a CE mark was applied to the system and it was successfully used for MRI-guided percutaneous interventions in 16 patients. Innomedic was purchased by Synthes [163] in 2008 and is no longer on the market; the reason is not known.

Onik et al. [164,174] developed a floor mounted stereotactic frame with nine, partially redundant, manually adjustable DOFs to place and hold a needle guide for CT-guided procedures. The frame is aligned with the transversal plane using the imager integrated laser system. The needle guide is positioned to intersect with the retrieved skin entry point and rotated to an angle in accordance with the planned needle path before manual insertion. In a clinical evaluation of the system, 23 out of 25 lesions were reached after the first needle manipulation. The procedure time, number of needle manipulations and number of image scans for localization decreased. Two more manually adjustable stereotactic frames to place and hold a needle guide were patented by Koutrouvelis [165] and Bidwell [166], both for CT-guided needle placement.

The MRI-guided system developed by Hata et al. [167] allows manual orienting of a needle guide on an automated 3-DOFs XYZ stage registered with the imager coordinate system using an optical tracking system. When manually orienting the needle guide, the three translational stages automatically translate the needle guide such that the axis of guidance of the needle guide remains intersecting a predetermined target location in the body. Thus, a virtual remote centre of motion is created at the target location and the physician is enabled to manually adjust the needle path towards the target using the visual feedback of a graphical user interface. After choosing an appropriate needle path the needle is inserted manually. Two encoders measuring the lateral orientation of the needle guide are used as feedback for control of the three translational stages. The assistance of the system was found effective in a clinical test of MRI-guided microwave coagulation of liver tumours in 15 patients [175].

Chinzei et al. [168] developed an MRI compatible actuated instrument guidance system mounted above the imager table in between two vertical MR coils. The system contains five DOFs and consists of a vertical linear stage which positions two planar stages which in turn manipulate a needle guide via two parallel slender rods, similar in concept to the iSYS Medizintechnik [151] iSYS1 [152].

The systems by Perfint $[169,170]$ are mobile robots on wheels which can be manually positioned next to the table of a CT scanner onto a docking station calibrated with the scanner. The systems are capable of automatically positioning and orienting a needle guide according to path planning, using a series of two 


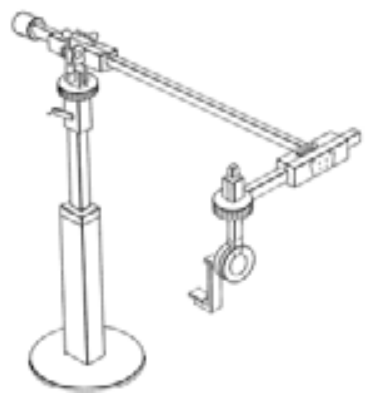

(a) Onik et al. Illustration adapted from [164].

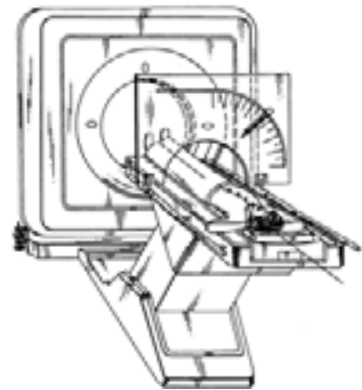

(c) Bidwell. Illustration adapted from [166].

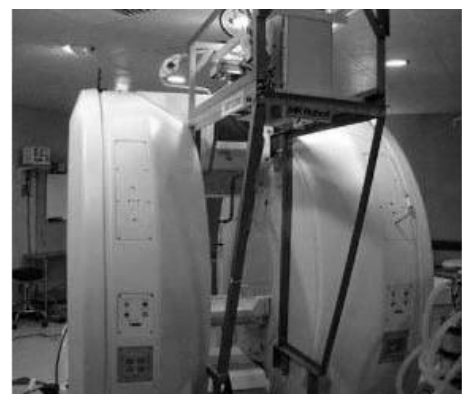

(e) Chinzei et al. Illustration adapted from [168]. Copyright (C) 2000 by Springer-Verlag Berlin Heidelberg. Reproduced by permission of Springer-Verlag Berlin Heidelberg.

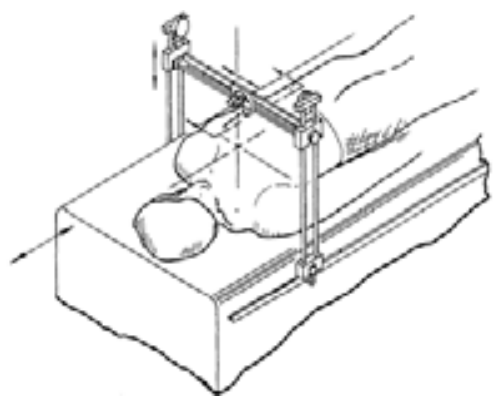

(b) Koutrouvelis. Illustration adapted from [165].

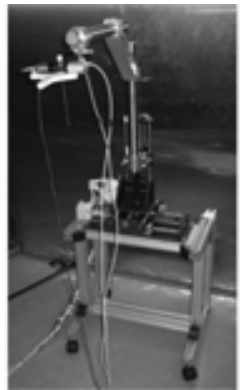

(d) Hata et al. Illustration adapted from [167]. Copyright (c) 2008 by Wiley-Liss, Inc. Reproduced by permission of Wiley-Liss, Inc.

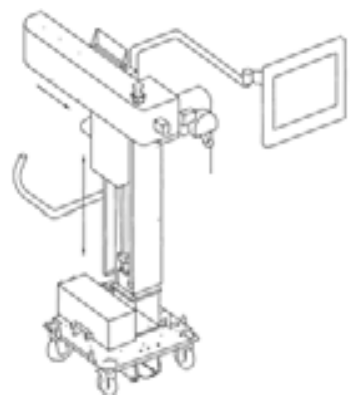

(f) Perfint [169] ROBIO. Illustration adapted from [170].

Figure 2.7: Gantry and floor mounted systems that supply physical guidance. 


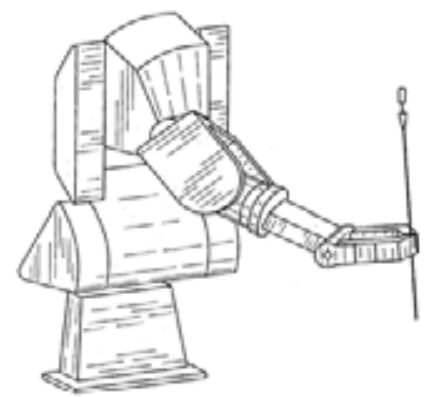

(g) Yanof et al. Illustration adapted from [171].

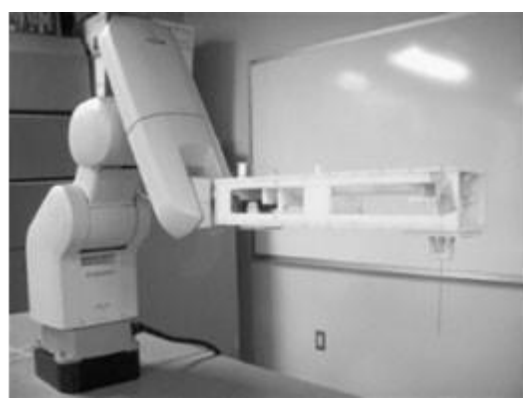

(h) Zhou et al. Illustration adapted from [172]. Copyright (c) 2012 by John Wiley \& Sons, Ltd. Reproduced by permission of John Wiley \& Sons, Ltd.

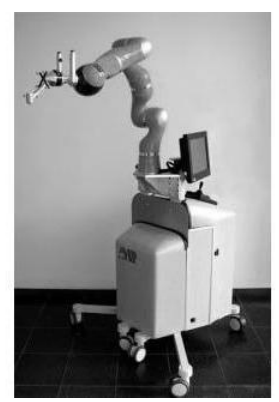

(i) Tovar-Arriaga et al. Illustration adapted from [173]. Copyright (C) 2011 by John Wiley \& Sons, Ltd. Reproduced by permission of John Wiley \& Sons, Ltd.

Figure 2.7: Gantry and floor mounted systems that supply physical guidance continued.

or three orthogonal linear stages and two orthogonal rotational stages. The end effector of the system is an automated gripper for a disposable needle guide. Insertion of the needle through the guide is performed manually, after which the gripper can be opened for release. Registration of the system with respect to the CT scanner coordinate system is performed through fiducials on the docking station which are captured by a camera in the bottom of the mobile system and a dual axis tilt sensor.

The same CT-integrated articulated robot used by Wood et al. [128, 129] was previously equipped with a pneumatic needle gripper to automate needle placement including automated needle insertion by Yanof et al. [176]. A load cell was later added to the needle gripper to allow for haptic control on needle insertion $[171,177]$. 
A Mitsubishi RV-E2 6-DOFs articulated robot was used in a setup by Zhou et al. [178] to investigate CT-guided automated needle placement for biopsy of lung nodules during respiratory motion. The end effector of the floor mounted robot is a needle gripper at the tip of a $460 \mathrm{~mm}$ long acetal beam used to reach inside the imager bore. This is the only listed system in which manipulation of the needle is continuously compensated for respiratory motion which is tracked by a vision system. In a preliminary test, an 18 gauge needle was inserted 300 times along different trajectories up to $160 \mathrm{~mm}$ long in a phantom undergoing rigid body movement simulating respiration, resulting in a targeting accuracy of $0.5 \pm 0.1 \mathrm{~mm}[172]$.

Tovar-Arriaga et al. [173, 179] present a system consisting of a 7-DOFs DLR/KUKA Light Weight Robot III equipped with a needle guide and registered with an angiographic C-arm using an optical tracking system. The system offers various placement methods, including automated needle guide placement according to planning with subsequent manual adjustment of the skin entry point through remote control, while maintaining its aim at the chosen target for the needle tip, a function similar to that of the system by Hata et al. [167].

\subsection{Discussion}

An overview of over forty existing systems for image-guided percutaneous needle placement in the torso has been presented, ranging from simple passive aids, with minimal interference in existing workflows, to fully actuated imagerintegrated robots, which take over full control of needle placement.

Navigation systems can be used to provide feedback on needle placement to the physician through a graphical user interface. Manipulation of the needle by the physician makes this type of system versatile yet also user-dependent. While these systems occupy relatively little space directly around the patient, they do have a footprint in the imaging suite and require an unobstructed line of sight or an undistorted magnetic field. The ActiViews [81] CT-Guide [82] offers a cheaper alternative that effectively eliminates this issue with a tiny, disposable system.

Systems that use the direction of gravity as a reference to orient a needle can also be relatively small, simple and low cost, with minor impact on existing workflows. The INRAD [105] AccuPlace [106] is one of the most simple and compact of all discussed systems. However, this type of system requires manual alignment with both the direction of gravity and the imager table or gantry during needle insertion.

Systems that project a laser as an axial reference can be effective with little interference with existing workflows. Alignment of the axial reference according to the planned trajectory is fully separated from needle manipulation, facilitating needle placement. Furthermore, there is no physical contact at any point in the procedure between the system and the needle or the patient, avoiding additional sterilization procedures. A 6-DOFs robot, as used by Wood et al. [128, 129], is a 
complex way to manipulate the 4-DOFs laser reference. Taking advantage of the imager gantry tilt and imager table translation, the Amedo LNS [114, 115, 127] needs only two automated imager-integrated DOFs to accomplish equal functionality. The commercially available Neorad [112] SimpliCT [113,123-126] evidences a particularly streamlined design, that wheels away when not in use.

Patient mounted systems can be very small, simple and low-cost, as illustrated by the designs of the two commercially available systems Neorad [112] Simplify [131] and the Apriomed [132] Seestar [133, 138, 139], and the actuated Robopsy system [134,140,141]. Attempts to create 5- or 6-DOFs actuated patient mounted systems have led to rather unusual and complex designs which occupy a large volume of an already limited space. Part of the rationale behind patient mounting is the assumption that the skin and target move as a rigid body, such that respiratory motion is inherently compensated for. The validity of this assumption however quickly expires with increasing needle insertion depth, due to the flexible body movement of the torso and sliding motion of abdominal organs with respect to the skin by respiration and the forces for physical guidance and insertion of the needle. Patient mounting however does provide inherent safety in case of patient movement, since system and needle freely move with the patient's skin. A weakness of patient mounted systems and systems which do not provide any physical guidance, is the application of forces to the torso upon needle insertion other than the axial component. This may result in tissue movement and deformation and, consequently, needle placement errors. Table, gantry and floor mounted systems avoid this by transmitting the guidance forces through a stiff mechanical construction. These however generally occupy more space and their usage may hinder patient access and require changes to the current workflow. While this is the largest category of systems, only the iSYS Medizintechnik [151] iSYS1 [152] and Perfint $[169,170]$ systems are currently commercially available.

Imager compatible systems that supply physical guidance and active needle insertion can be remotely controlled to provide the opportunity of needle manipulation under real-time imaging. This avoids the need for a physician to enter the imaging field, which is confined by the imager bore and hazardous in case of radiological imaging. Real-time imaging is especially useful for continuous monitoring during high risk needle insertions. In systems with active needle insertion, haptic control should be integrated to account for the demands of a physician to feel needle tissue interaction, e.g. as experimented with in the system by Yanof et al. $[171,176,177]$ or the CT-Bot $[136,143,144]$. In addition, more advanced systems could automatically adapt for respiratory motion during insertion to avoid the need for breath holding, as proposed e.g. by Zhou et al. [172,178], or compensate for tissue movement and needle deflection by implementing means for needle steering to further reduce placement errors. However, such advancements are still in the research stage and will likely lead to increased system costs and complexity, from a technical point of view, as well as with regards to product certification, which may outweigh the benefits.

The presented overview shows that there is a wide variety of developed sys- 
tems with growing complexity. However, given that only few systems have reached commercial availability, it is clear that the technical community is struggling to develop solutions that are clinically adopted. The question rises: why is this so?

In the authors' personal experience, freehand percutaneous needle placement is generally perceived by clinicians as sufficient to achieve satisfactory patient outcomes. While improved accuracy is a goal of many systems, it has not been clearly demonstrated that the accuracy obtained by clinicians' manual skill is a limiting factor to procedural success in terms of patient outcome. Furthermore, freehand needle placement employs minimal equipment and image-guided procedures typically reimburse at fixed amounts. Adding any systems into the procedure is therefore rightfully seen by healthcare providers as increasing complexity and costs.

To emphasize the added value of a system, we recommend the development of a comprehensive standard method of assessing and reporting the performance to cost ratio, taking into account patient outcome, time for needle placement, time for system installation, equipment costs and sterilization procedures amongst others. Technically relevant parameters such as needle properties, insertion angles and depth, penetrated tissue layers, $\mathrm{X}$-ray exposure (if applicable) and number of iterations and final placement errors should be uniformly defined and clearly specified. The results of freehand needle placement at multiple clinical centres should act as a benchmark. This enables the technical community to generate a complete and adequate set of system requirements and enables the medical community to evaluate the overall merit of a specific system.

In conclusion, it is clear that none of the systems discussed, while many are technically sound, represent a breakthrough in percutaneous procedures on par with that of the imaging technology that made them possible. Thus, there is still open space for innovation and readers are invited to draw their own conclusions as to the art's direction. 

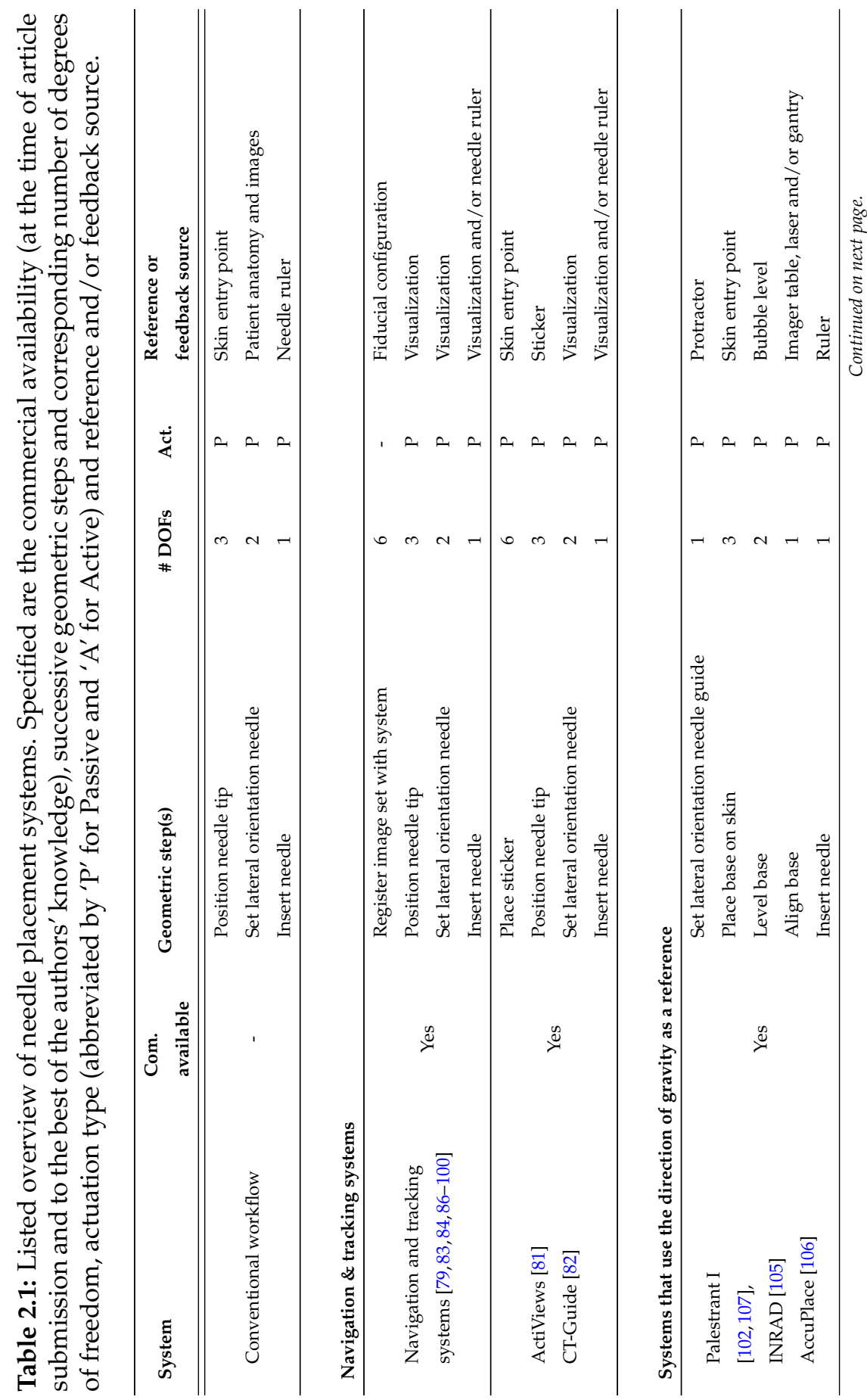


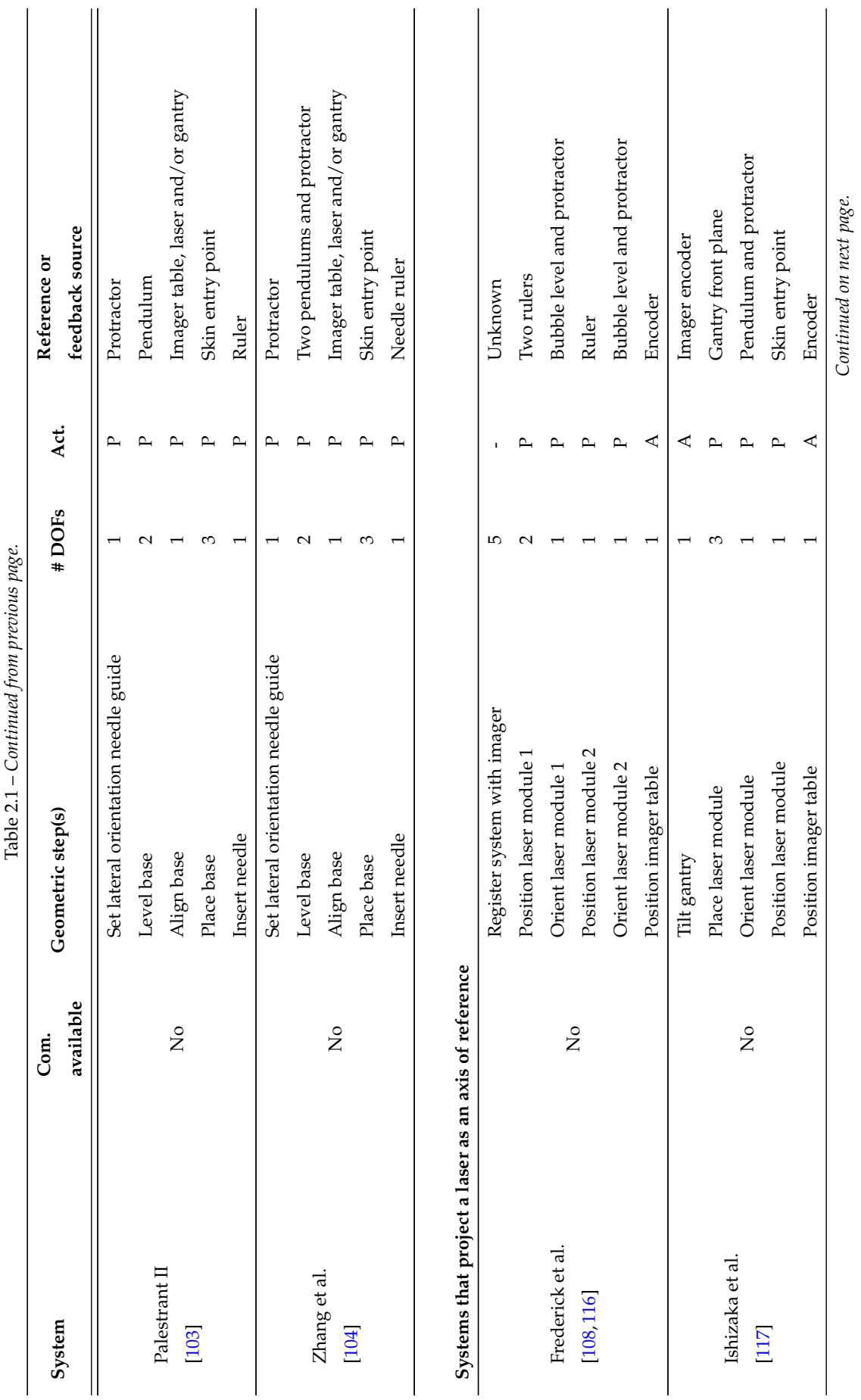




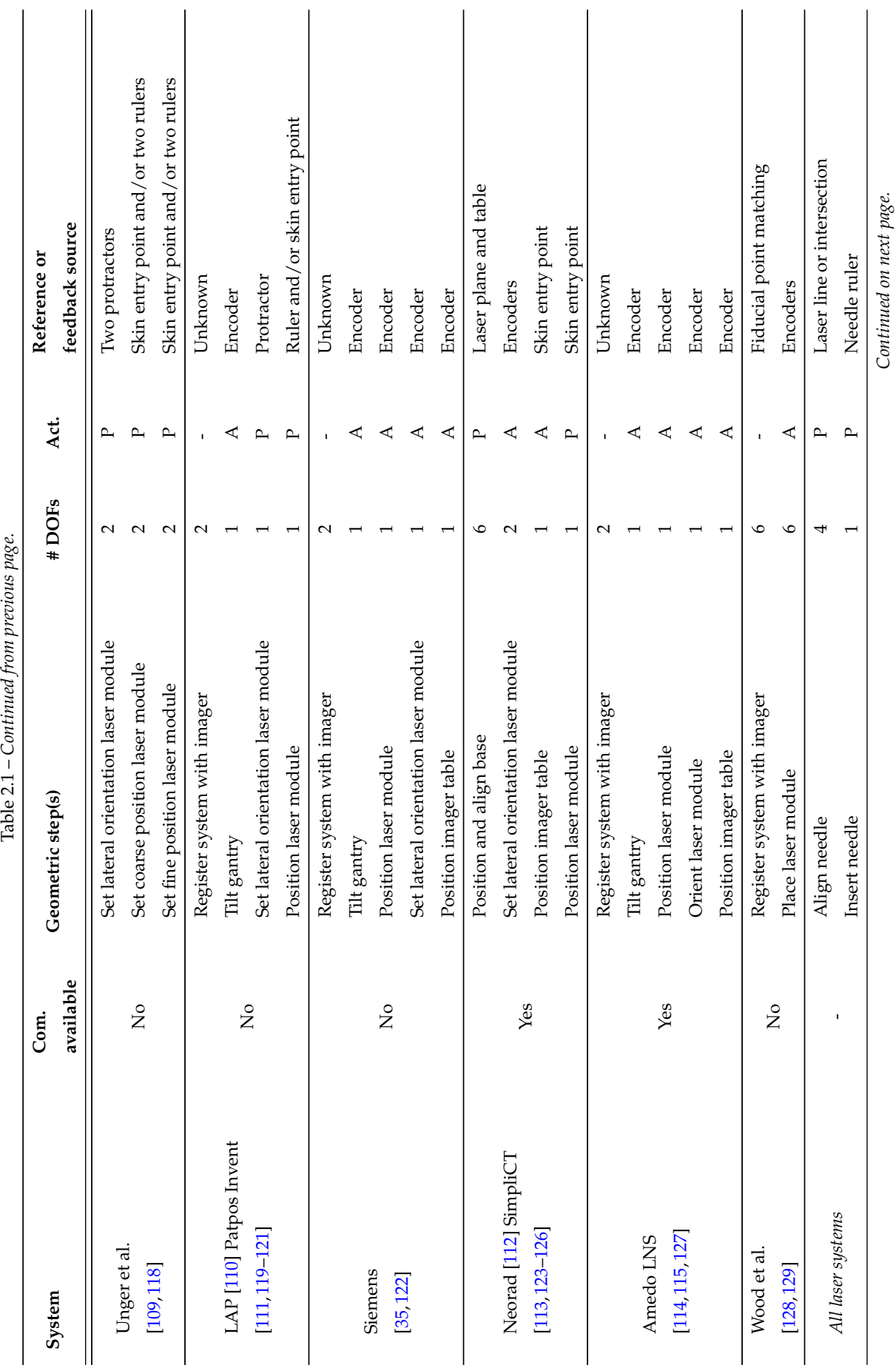




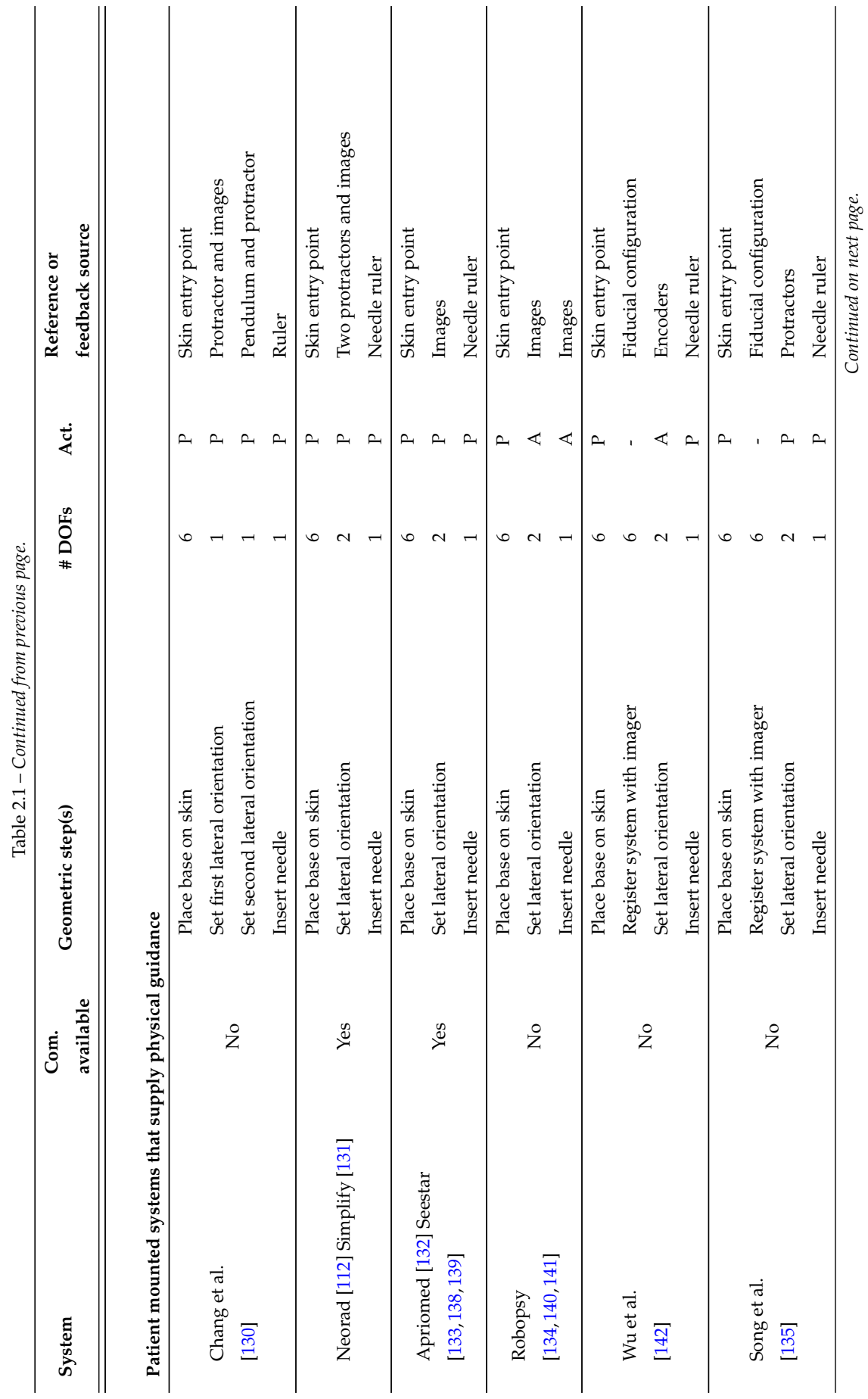




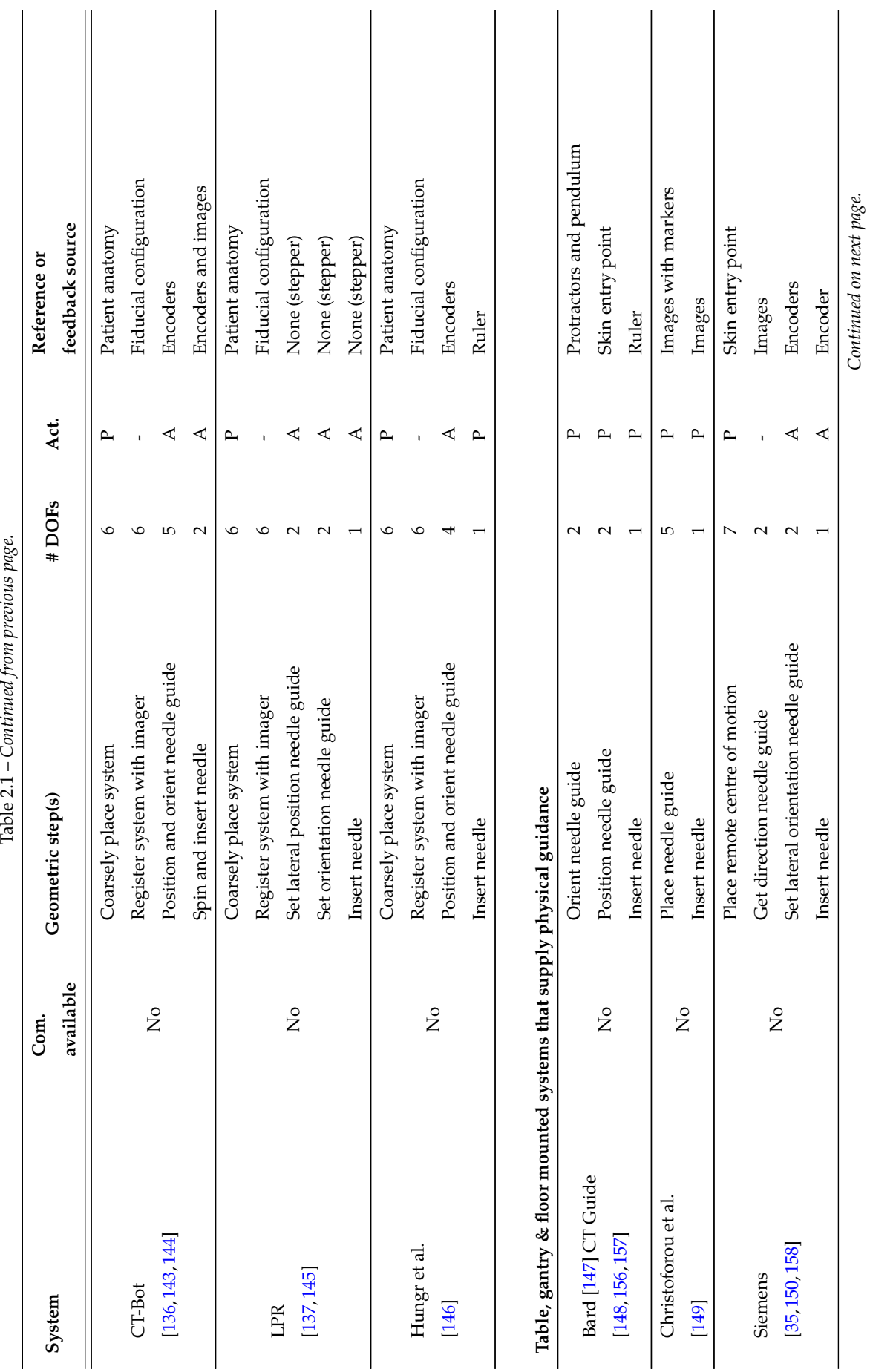




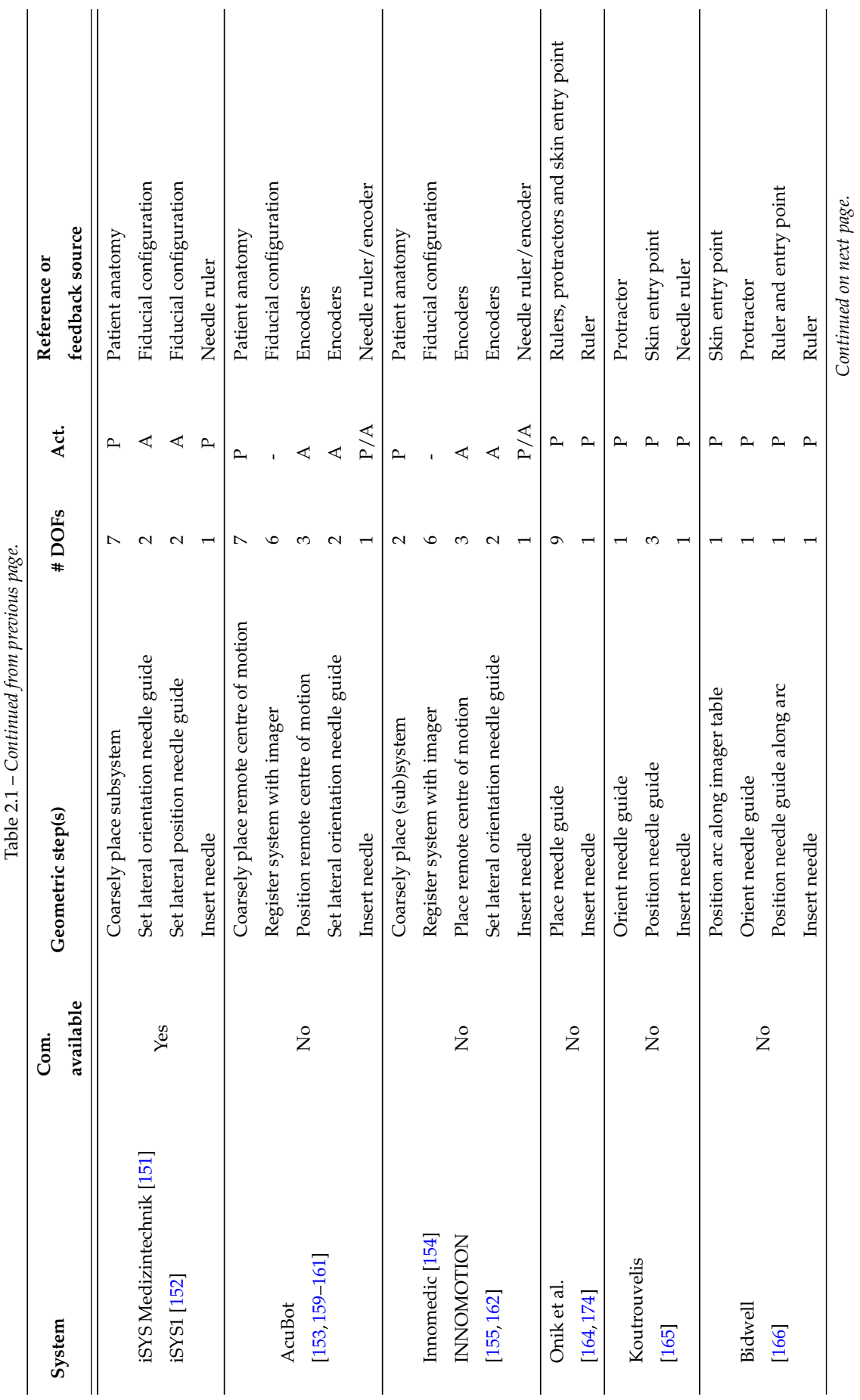




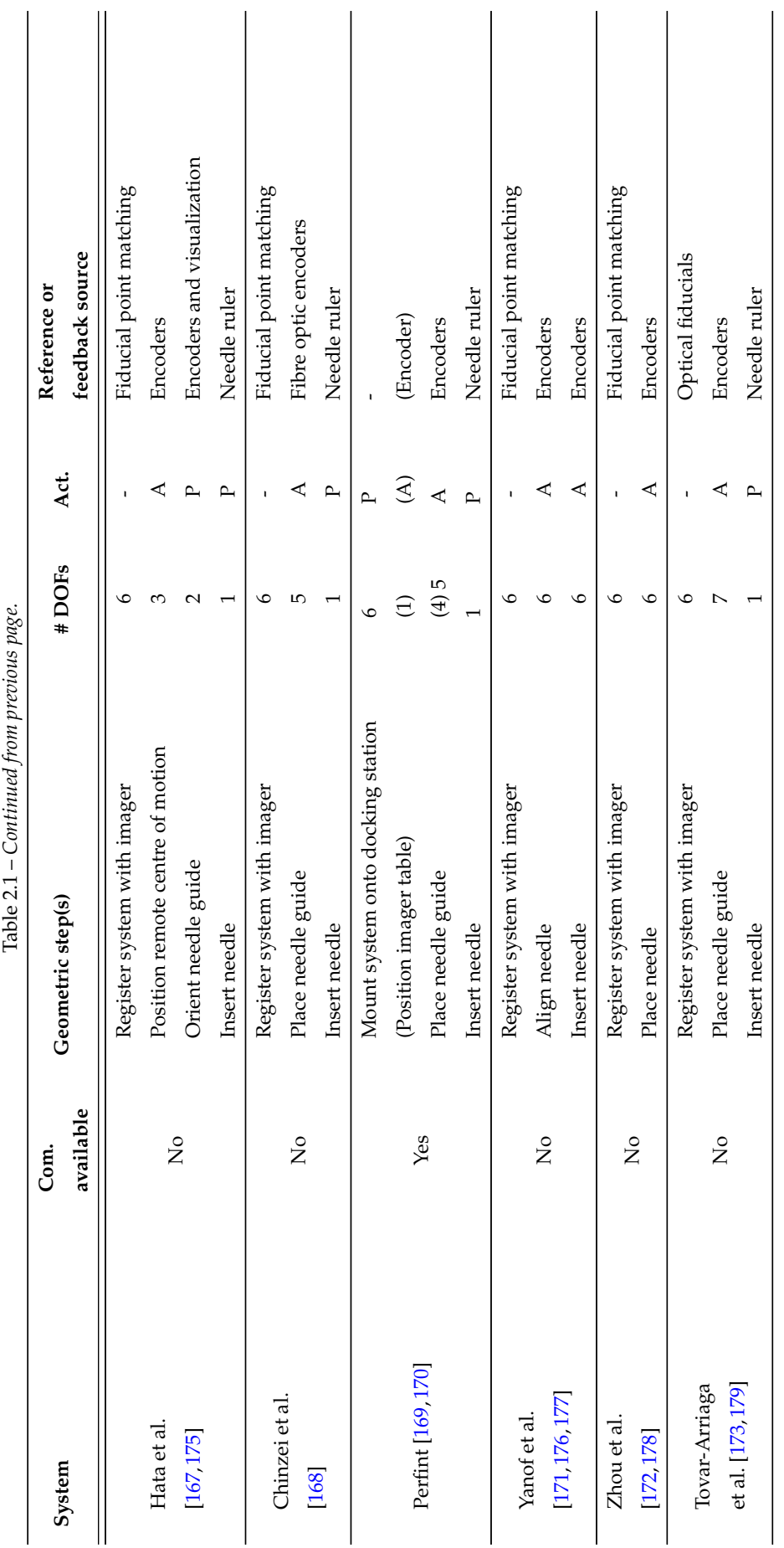




\title{
CHAPTER 3
}

\section{System for CT-guided needle placement in the thorax and abdomen - a design for clinical acceptability, applicability and usability}

\author{
Maarten M. Arnolli, Martijn Buijze, Michel C.J. Franken, \\ Koert P. de Jong, Dannis M. Brouwer and Ivo A.M.J. Broeders \\ Under review with the International Journal of Medical Robotics and Computer \\ Assisted Surgery, John Wiley E Sons, Ltd.
}

\begin{abstract}
Background Various systems exist for CT-guided needle placement in the thorax and abdomen, but widespread adoption is lacking. A novel system has been designed for clinical acceptability, applicability and usability.

Methods Based on the workflow and conventional freehand method for needle placement, a system was outlined which incorporates a needle guide on a remote centre of motion mechanism, freely placeable around the patient at the desired entry point and push-button lockable to the CT table. System and patient are scanned for system-to-CT registration and target specification. The needle guide is automatically aimed at the target, for manual needle insertion to specified depth.
\end{abstract}

Results The functionality of a prototype was demonstrated for 24 in- and out-of-plane punctures in a gelatin phantom, achieving $1.2 \pm 0.6 \mathrm{~mm}$ and $2.1 \pm 0.7$ $\mathrm{mm}$ placement error at $79.0 \pm 8.4 \mathrm{~mm}$ and $156.0 \pm 6.9 \mathrm{~mm}$ depth.

Conclusions The system enables precise needle placement in a single insertion, in compliance with $\mathrm{a} \leqslant 5 \mathrm{~mm}$ clinical user requirement. 


\subsection{Introduction}

Minimally invasive biopsy and ablation of tumours in the soft tissues of the abdomen and thorax are typically performed via a needle which is inserted percutaneously ('through the skin') using image-guidance to reach the target tissue. X-ray spiral computed tomography (CT) is a widely used imaging modality for needle placement in the liver [38-40] and lungs [42,45, 180]. Use of magnetic resonance imaging (MRI) for these procedures is less prevalent than CT [69], but feasibility has been shown in the liver $[63,64,66]$ and lungs $[63,68]$. CT- and MRIguided needle placement are conventionally performed freehand by the physician. This method is user-dependent and generally requires multiple iterations of needle placement and intermediate imaging to achieve satisfactory placement. Each placement iteration increases tissue damage with accompanied risks, patient X-ray exposure in case of CT, procedure time and costs.

A variety of systems has been developed over the past decades to improve the procedure of CT- and/or MRI-guided needle placement, ranging from simple passive aids to fully automated robots [72]. Exemplary systems providing only visual guidance for needle placement are the needle navigation systems ActiViews [81] CT-Guide and CAScination [92] CAS-ONE, and the laser reference systems Neorad [112] SimpliCT and Amedo [114] LNS. Systems providing physical needle guidance include the patient mounted AprioMed [132] Seestar, the Light Puncture Robot [145] and Robopsy [140], the table mounted AcuBot [159], INNOMOTION [155], and iSYS Medizintechnik [151] iSYS1 and the floor mounted Perfint [169] ROBIO and MAXIO and various articulated robotic arms $[173,176,178]$. Despite the various attempted strategies and often technically sound solutions, widespread clinical adoption of any system is lacking and open space for innovation remains.

For all needle placement systems, getting the needle from point $A$ to $B$ is a technical prerequisite. A challenge lies in the development of a clinically acceptable, widely applicable and usable system that enables user-independent, precise needle placement in a single insertion, using lower radiation exposure and shorter procedure time. Our goal is the development of such a system. It can be achieved by wisely choosing functionality, by thought-through user interfacing and by integration in and improvement of the existing clinical workflow, while introducing minimal substitutive user effort in return. Based on this philosophy, a novel system for CT-guided percutaneous needle placement in the thorax and abdomen was developed (Fig. 3.1). This article discusses the subsequent stages of the development process and presents the elaborated design, focusing on its functional aspects relevant to clinical use, and a performance assessment of a first prototype. 


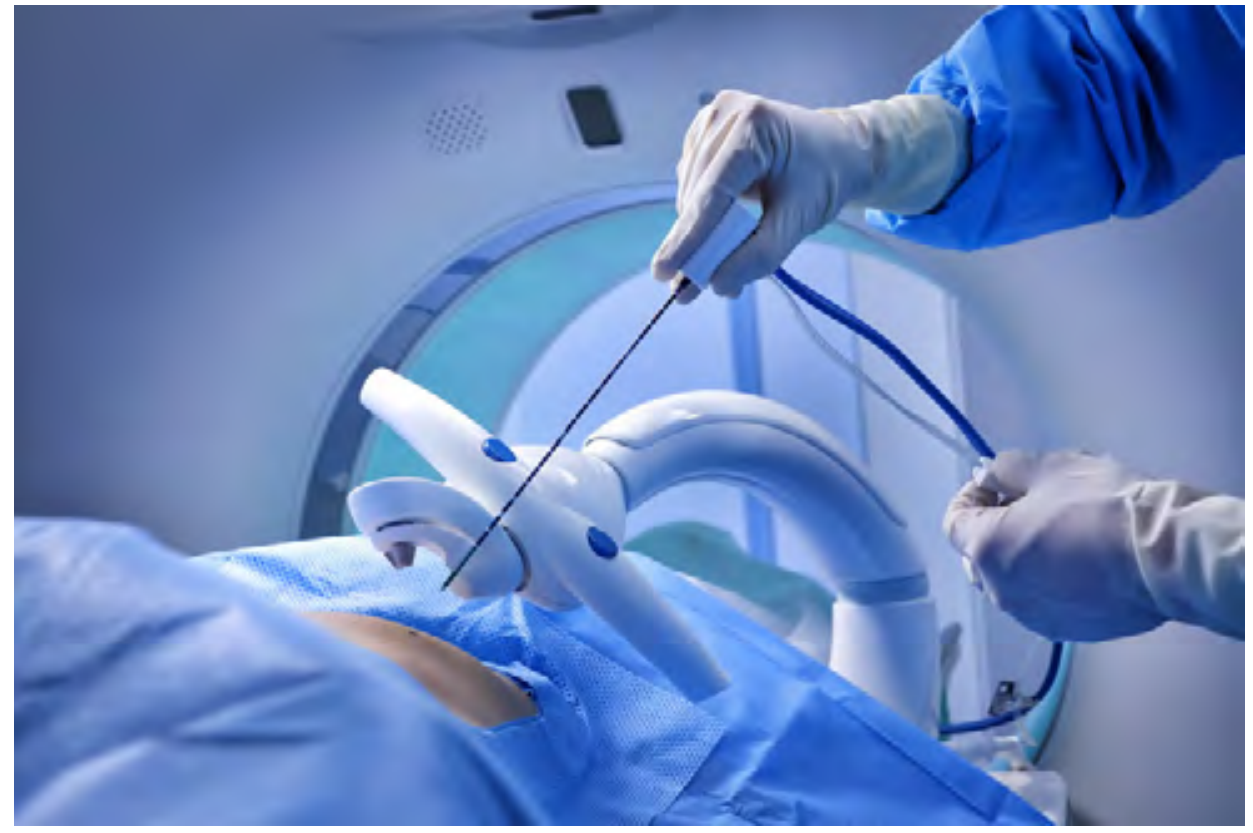

Figure 3.1: Prototype of the novel system for CT-guided percutaneous needle placement in the intervention suite.

\subsection{Materials \& methods}

The development of the novel system has been executed in compliance with the requirements of the ISO 13485 and IEC 60601 standards. Our research started by visiting multiple medical centres, attending clinical procedures which involve needle placement, including radiofrequency (RFA) and microwave ablation (MWA) of liver tumours and lung nodule biopsies. A problem analysis of the freehand method and workflow was performed, identifying its strengths and weaknesses, and a comprehensive overview of the state of the art of needle placement systems was assembled [72]. User requirements for a novel system were defined. A novel placement method and adjusted workflow were composed, outlining the system functions and corresponding functional requirements as a the framework for design elaboration.

\subsubsection{The workflow of freehand needle placement}

The workflow of CT-guided freehand needle placement was observed during clinical attendances of liver tumour ablations and lung nodule biopsies at multiple medical centres in the Netherlands (Fig. 3.2). 


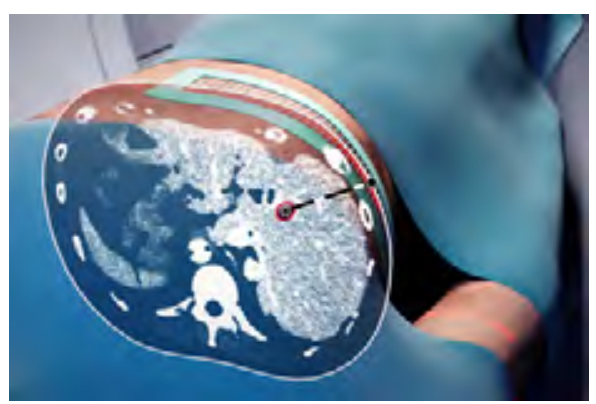

(a) CT scanning \& path planning from entry point to target.

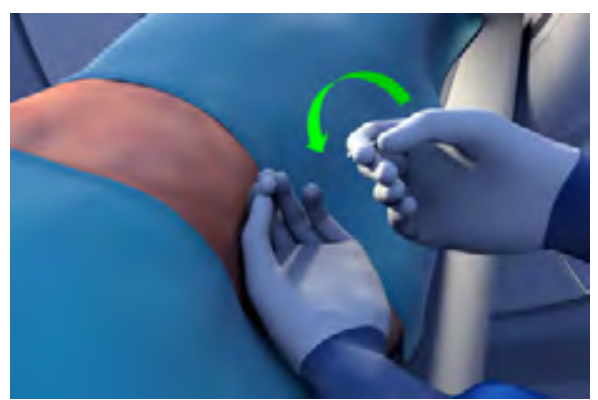

(c) Freehand needle aiming and superficial insertion.

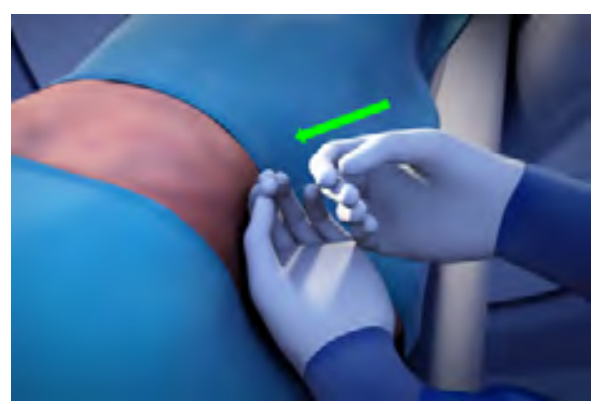

(e) Manual needle insertion towards the target.

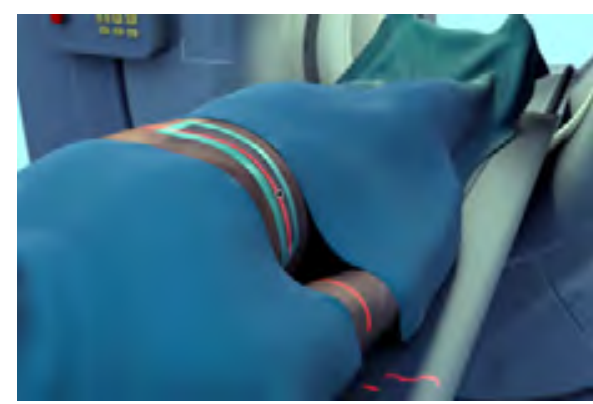

(b) Entry point retrieval using radiopaque grid lines and laser projection.

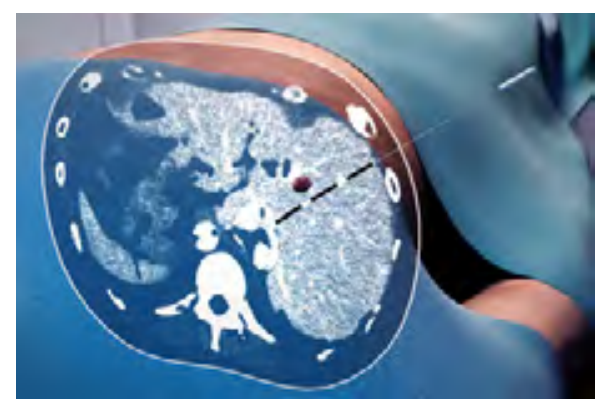

(d) CT scanning \& evaluation of the needle trajectory.

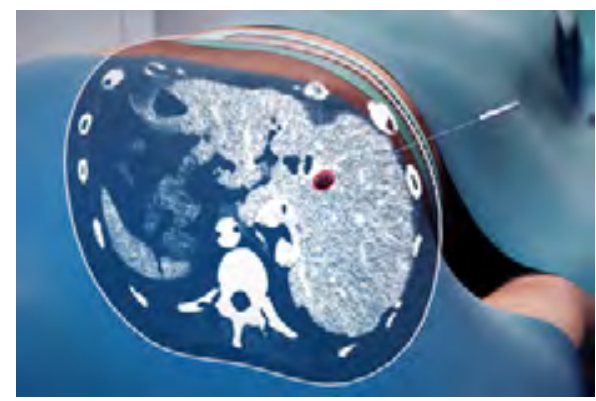

(f) CT scanning of the patient and needle for placement evaluation.

Figure 3.2: Illustrations of the workflow of CT-guided freehand needle placement in the liver.

CT-guided percutaneous procedures are performed in a dedicated intervention suite, consisting of an X-ray insulated main room in which the CT scanner is located and an adjacent control room. Upon arrival in the main room, the patient 
is laid down onto the CT table. The arms of the patient are commonly folded above the head to provide workspace around the torso, the feet pointing towards the CT bore. General anaesthesia and respiration control by a medical ventilator may be applied depending on the procedure.

The anatomical area of the intervention is known beforehand based on preoperative diagnostics. To create a sterile field, the skin area where the needle will enter is treated with disinfectant and sterile drapes are applied to cover the surrounding area. A sterile adhesive sheet of radiopaque grid lines is attached to the skin which will be used to retrieve the planned entry point of the needle. A radiocontrast agent may be injected intravenously to enhance the visibility of relevant structures. The medical staff must evacuate to the control room to avoid exposure to radiation during a first $\mathrm{CT}$ scan to acquire images of the relevant anatomical area. Respiration is paused in inhaled state during scanning. In a reconstructed set of parallel images, the physician locates the target and performs path planning of the needle, avoiding large blood vessels and impenetrable structures such as the ribs. This yields a target for the needle tip and an entry point on the patient's skin (Fig. 3.2a).

The next step is retrieval of the entry point. The CT table is automatically positioned such that the position of the image in which the entry point was planned coincides with the projected vertical plane of a laser incorporated in the CT gantry. The result is a laser line on the skin intersecting with the radiopaque grid lines also seen on the image. This enables the physician to pinpoint and mark the entry point with millimetric precision (Fig. 3.2b). The grid lines are removed afterwards.

Now, the insertion angles of the needle path need to be determined. The physician creates a mental map of the path with respect to the patient and the CT table and translates this to the actual setting. The needle is superficially inserted at the estimated angles (Fig. 3.2c). A shorter, lightweight hypodermic or trocar needle is commonly used at this point instead of the heavier biopsy or ablation needle. Local anaesthesia is applied if applicable. A minor skin incision may be created before insertion to avoid resistance and abrupt penetration and to prevent entrainment of epithelial cells.

Once again, the medical staff needs to evacuate and respiration is paused for a second CT scan, to evaluate the trajectory of the needle to the target (Fig. 3.2d). The required insertion depth with respect to the skin surface is determined using the image viewer and measured using linear markings on the needle. The biopsy or ablation needle is advanced towards the target based on the latest angular estimation (Fig. 3.2e), followed by yet another evacuation, respiratory pause and CT scan. If the needle is off target, it is retracted and reinserted at adjusted angles. It is not unusual for this process of adjustment, CT scanning and evaluation to take six or more iterations. Once the needle is finally placed to satisfaction (Fig. 3.2f), the physician can proceed to the original goal of the procedure: performing the biopsy or ablation. 


\subsubsection{Problem analysis}

Several steps of the described workflow leave for little improvement in terms of efficiency. Retrieval of the entry point after path planning is a straightforward process thanks to the use of grid lines and laser projection. Also, the insertion depth of the needle is easily determined and sufficiently accurately measured using the linear marks on the needle.

The prime opportunity for improvement is finding the right angles of insertion after retrieval of the entry point. Since no clear reference of the planned trajectory is available, the accuracy of the estimated angles is user-dependent and often poor, leading to erroneous needle placement. Also, manually supporting the needle to maintain its angles during insertion is a challenging task and is prone to cause tissue deformation and needle deflection, further increasing the placement error. The result is a cumbersome iterative method which yields unnecessary tissue damage, patient $\mathrm{X}$-ray exposure, time consumption and associated costs.

\subsubsection{User requirements}

The user requirements for a novel system for needle placement were defined based on the problem analysis and in close cooperation with medical experts. The principal user requirement is the following:

- The system should enable user-independent, precise needle placement in a single insertion, less radiation exposure and shorter procedure time.

Based on the clinical characteristics of the intended procedures, this is complemented by the following requirements:

- Placement error: the system should achieve needle placement with a tip to target error of less than $5 \mathrm{~mm}$, with a design goal of $2 \mathrm{~mm}$ up to an insertion depth of $250 \mathrm{~mm}$.

- Entry points: the system should be applicable to a range of entry points which covers the entire thorax and abdomen.

- Path angles: the system should be applicable to needle paths both coincident with and oblique to the transversal plane.

- Needle types: the system should be applicable to available needles used in current clinical practise for biopsy and ablation, with various diameters.

- Sterility: the system should not violate the sterile field of the procedure.

- CT compatibility: the system should not deteriorate the diagnostic quality of acquired CT images. 


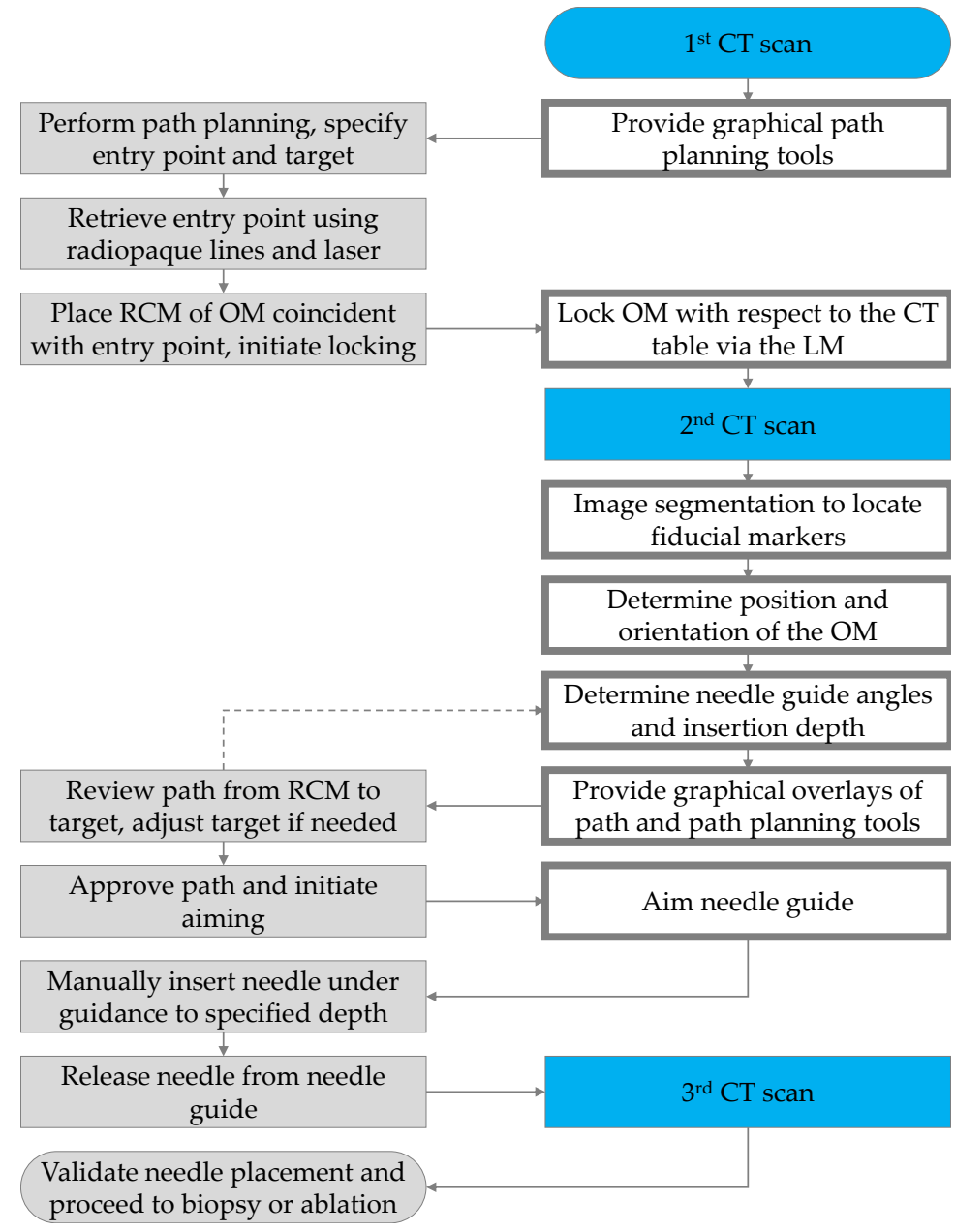

Figure 3.3: Flowchart of the novel needle placement method, indicating the functions of the physician on the left hand side and the functions of the CT scanner and system on the right hand side.

\subsubsection{Novel placement method}

A novel method for needle placement has been composed based on the problem analysis and user requirements (Fig. 3.3, Fig. 3.4). The strategy is characterized by two key principles. The first is that needle insertion remains a manual task of the responsible physician, to maintain inherent haptic feedback on needle-tissue interaction and to facilitate clinical acceptability. The second is that needle insertion is physically guided along the line that coincides with the entry point and target, 


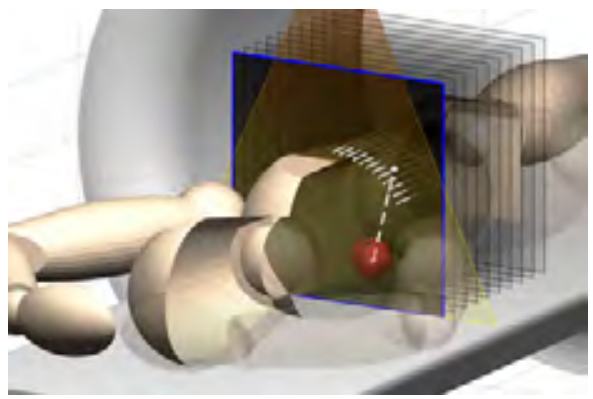

(a) $1^{\text {st }} \mathrm{CT}$ scan of the patient, initial path planning and entry point retrieval using radiopaque grid lines and laser projection.

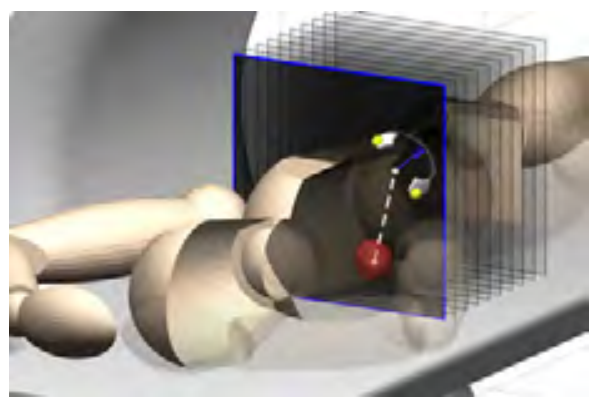

(c) $2^{\text {nd }} \mathrm{CT}$ scan of the patient and OM, image segmentation to locate fiducial markers, OM-to$\mathrm{CT}$ registration and target specification.

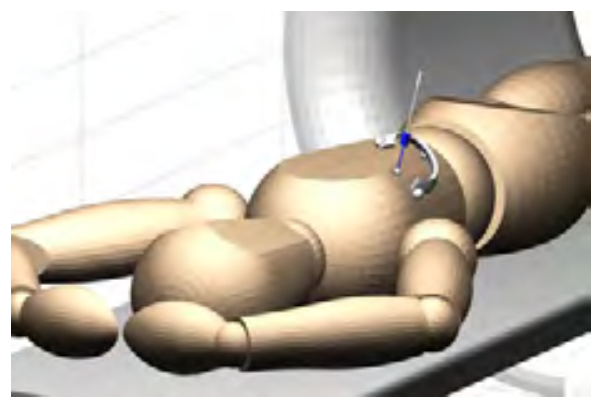

(e) Manual needle insertion through the needle guide to specified depth.

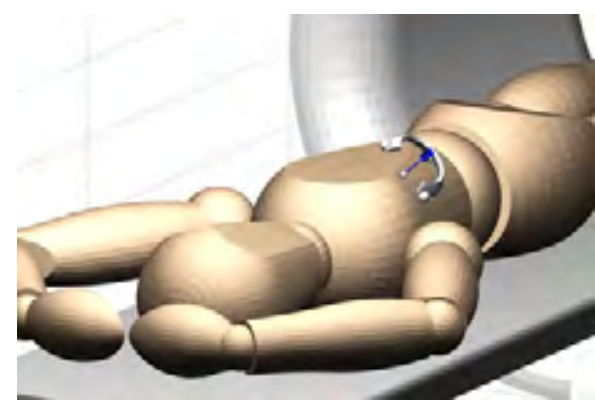

(b) Manual placement of the OM such that the RCM coincides with the entry point, and automatic locking to the CT table by the LM (omitted from illustration).

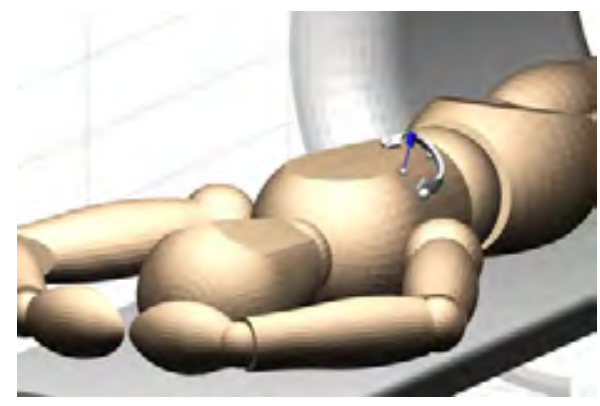

(d) Determination of the needle guide angles and insertion depth and automated alignment of the needle guide with the target.

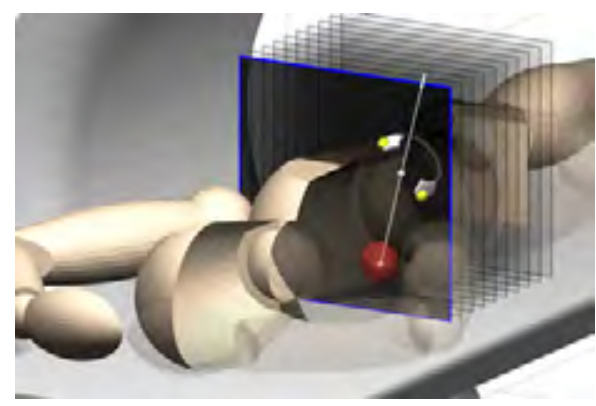

(f) $3^{\text {rd }} \mathrm{CT}$ scan of the patient and needle for placement evaluation.

Figure 3.4: Conceptual illustrations of the novel needle placement method. 
to maintain the right direction during insertion and eliminate external loads other than axial on the needle, minimizing tissue deformation and needle deflection.

The existing workflow is initially followed, performing a first CT scan under paused respiration to acquire a set of images, path planning and entry point retrieval using radiopaque grid lines and laser projection (Fig. 3.4a). At this point, a module of the novel system referred to as the orientation module (OM) is introduced, which incorporates a two degrees of freedom (2-DOFs) mechanism to aim a needle guide about a remote centre of motion (RCM). The 2-DOFs to set the direction of the needle guide plus 1-DOFs insertion enable 3-DOFs placement of a needle tip from the RCM. The OM is placed manually by the physician such that the RCM coincides with the entry point (Fig. 3.4b). After placement, the physician activates automatic locking of the OM to the CT table by a mechanical link, referred to as the locking module (LM).

A second image scan under paused respiration is performed, covering both the patient and the locked OM (Fig. 3.4c). The OM incorporates geometric references at known positions, referred to as fiducial markers. The positions of these fiducial markers are located by segmentation of the CT images and enable determination of the position and orientation or registration of the OM with respect to the CT coordinate system. Visual overlays of the path from the RCM to the initially specified target on both sets of CT images are provided for review by the physician. The target can be adjusted if needed. The corresponding angles of the needle guide and insertion depth are automatically calculated. The needle guide is automatically aligned accordingly by the 2-DOFs mechanism after approval from the physician (Fig. 3.4d).

The physician now manually inserts the needle through the needle guide under paused respiration and using the linear markings on the needle to measure the insertion depth with respect to the needle guide (Fig. 3.4e). After insertion, the needle is released from the needle guide to allow for the needle to move with the continued respiratory motion of the patient. Needle placement is evaluated on the images of a third and final CT scan of the patient and needle (Fig. 3.4f), after which the physician can proceed to the biopsy or ablation.

\subsection{Results}

This section presents the results of the detail design phase of the development process and a performance assessment of the novel system.

\subsubsection{Detail design \& prototype}

The detail design of the novel system was elaborated based on the user requirements, placement method and system functions. Figure 3.5 shows a computer aided design (CAD) model of the system, patient and CT scanner, with labelled 


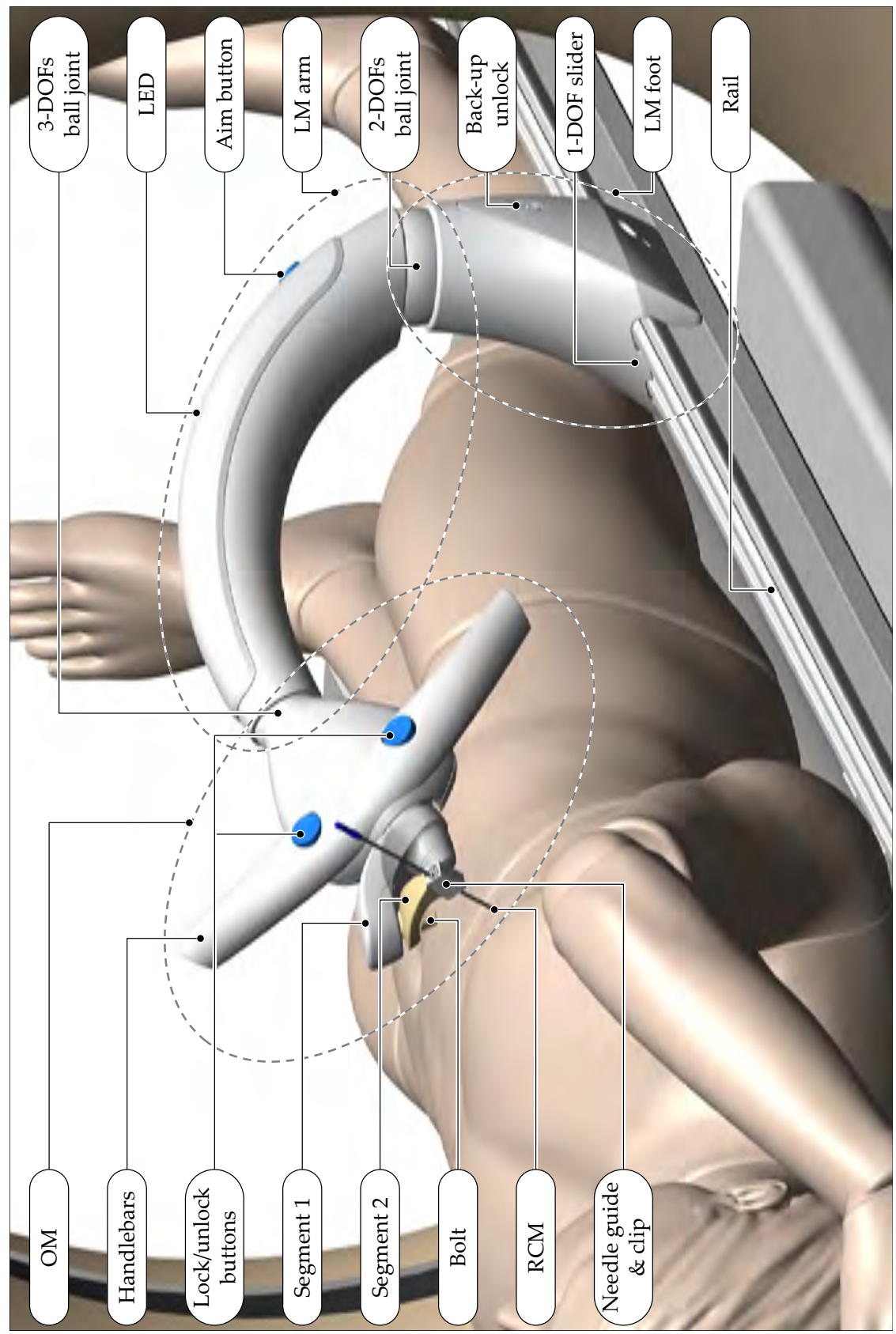

Figure 3.5: Impression of the system and patient in a CT scanner, with labelled components. 
components. A prototype was realized (Fig. 3.1). This section describes the various components of the system, focusing on the functional aspects relevant to its clinical use.

\section{The orientation module}

The primary function of the $\mathrm{OM}$ is to orient the needle guide about the RCM to align it with the target. This is performed by a 2-DOFs serial mechanism consisting of segment 1 connected via a revolute joint to the main body of the OM, and segment 2 connected via a revolute joint to segment 1 . The needle guide is incorporated in segment 2 . The two joint axes and the axial trajectory of the needle guide coincide in the same point outside the OM, creating the RCM. The angles of the needle guide are kinematically coupled via the segment angles to the two joint angles, which are actuated by electric servomotors. Worm gear transmissions prevent backdriveability of the needle guide, such that the needle guide maintains its angles in case of a power failure. The non-backdriveability is warranted up to a limited torque by slipper clutches incorporated in the drive system to both segments, to avoid the hazard of entanglement.

Precise axial needle guidance without play is achieved by a V-shaped groove integrated in segment 2 , in which the needle is pressed by a needle clip. Removal of the needle clip releases the needle. The needle guide accommodates needles of 1 to $4 \mathrm{~mm}$ in diameter. An RCM pointer is placed in the needle guide to indicate the position of the RCM during placement of the OM.

Four spherical $\varnothing 20 \mathrm{~mm}$ diameter fiducial markers of relatively radiopaque aluminium oxide are incorporated in the main body of the $\mathrm{OM}$ for registration with the CT coordinate system [181].

\section{The locking module}

The function of the LM is to lock the position and orientation of the OM with respect to the CT table after manual placement. The LM is a serial linkage consisting of a 1-DOF rail connected to the CT table, a foot, a 2-DOFs ball joint, an arm and 3-DOFs ball joint connected to the OM. In unlocked state, it allows for 6-DOFs manipulation of the $\mathrm{OM}$ within the $\mathrm{CT}$ bore and anywhere around the patient. A gravity compensating spring mechanism is integrated in the 2-DOFs ball joint to allow the OM to hang freely without falling. A pneumatic-hydraulic system is incorporated to automatically lock each of the three joints, and consequently the OM. A buffer in the pneumatic system ensures that the OM remains locked in case of any power failure. A pressure sensor in the hydraulic system is used to monitor the state of the locking system. 


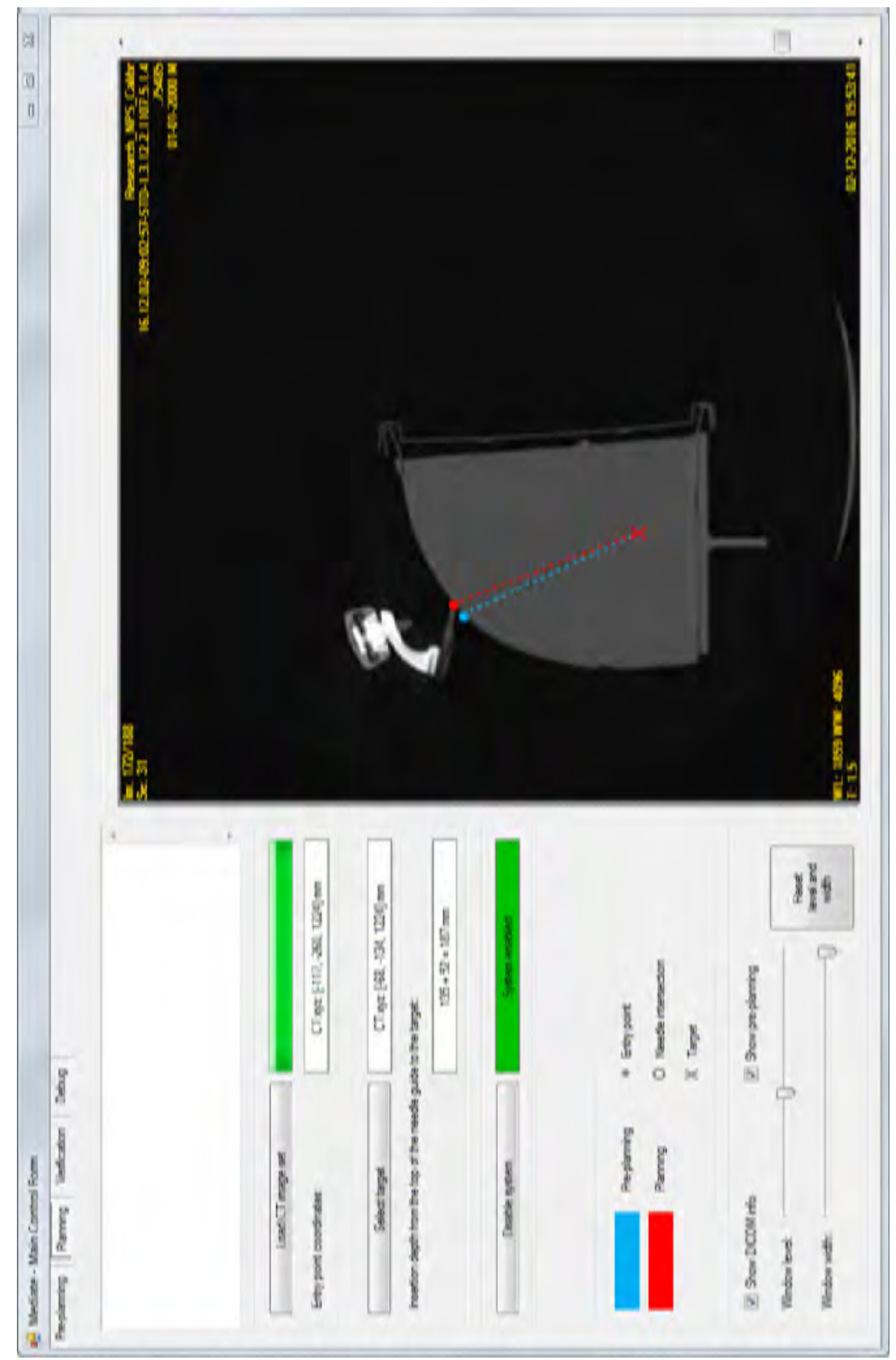

Figure 3.6: The planning tab of the GUI, containing controls on the left and a DICOM viewer with superimposed planned paths on the right. 


\section{User interfaces}

The user interfaces of the system are crucial for clinical usability. Two handlebars extend from the main body of the OM for manual placement. Two electrical push-buttons on the handlebars control the pneumatic-hydraulic locking system: pressing either button instantly locks the OM; pressing both buttons simultaneously unlocks it. This facilitates left-, right- or both-handed placement and locking, and mitigates the risk of unintentional unlocking by accidentally pressing a button. A back-up unlock button is incorporated in the LM foot to mechanically intervene directly in the locking system and ensure the system can be safely unlocked and removed in any case of electrical control failure.

Part of the prototype is a software application referred to as the graphical user interface (GUI) (Fig. 3.6). The GUI contains a pre-planning tab to perform path planning by using overlays on the images of the initial CT scan. In the planning $\mathrm{tab}$, the planned path is automatically updated to coincide with the RCM position determined using the OM-to-CT registration based on the second CT scan. An overlay of the updated path can be shown on the images of the initial CT scan in the pre-planning tab and vice versa, to take full advantage of all planning and image data for definitive target specification. The GUI furthermore provides the calculated insertion depth from the top of the needle guide to the target.

An electrical push-button is located on the LM arm to initiate aiming of the needle guide. Activation of this button requires a successful OM-to-CT registration, a user-specified target and user approval through the GUI. For safety concerns, releasing the button instantly stops movement of the needle guide. A blue light-emitting diode (LED) on the LM arm is switched on once the needle guide is correctly aimed, indicating the system is ready for needle insertion. Proceeding to aiming is blocked in case the OM was unlocked after loading the images, which is constantly monitored using the pressure sensor in the hydraulic system.

\section{System shape, size \& action range}

The design is shaped and sized to fit a $60 \mathrm{~cm}$ scanner bore, in consideration of future compatibility of the system with MRI. Most CT scanners have larger bore holes of 70 to $80 \mathrm{~cm}$ bores, leaving for extra space. The maximum width of the system in the transversal plane is $7.5 \mathrm{~cm}$, to minimize the limitation on maximum patient size. The rail can be on either the left or right side of a patient to create two partly overlapping ranges for placement of the OM. These cover a $45 \mathrm{~cm}$ diameter cross section along full length of the CT table, to maximize the clinical applicability of the system. The outer shape of the system is closed and rounded for safety and secure cleaning. Segments 1 and 2 have arc angles of $60^{\circ}$ and $52.5^{\circ}$ respectively, creating a full $90^{\circ}$ conical action range of the needle guide trajectory (Fig. 3.7). 


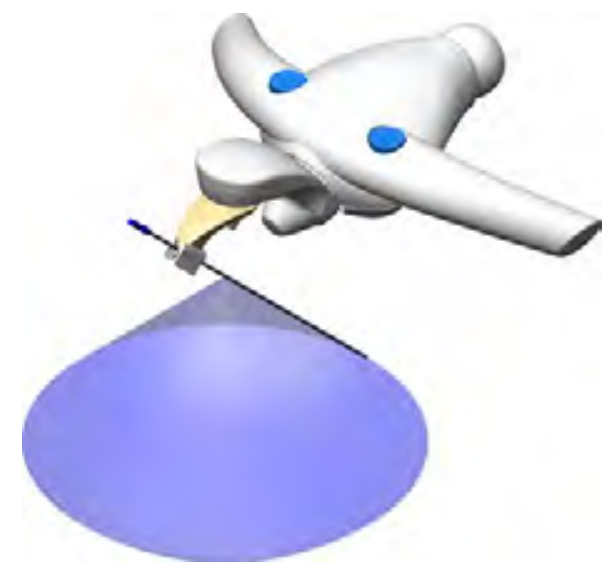

(a) Needle $45^{\circ}$ to the back.

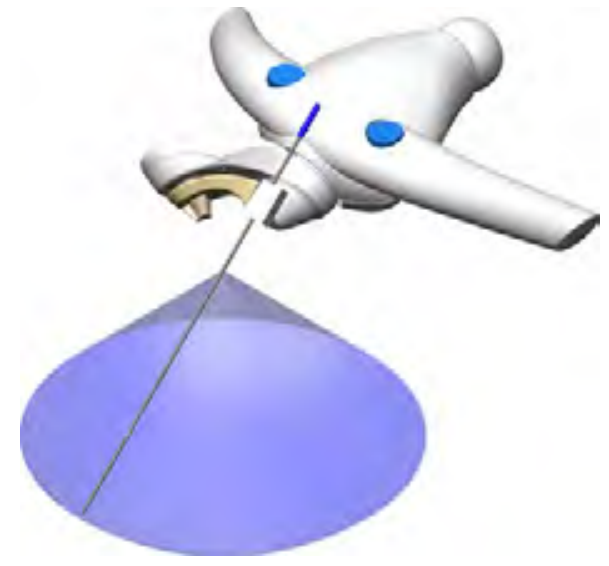

(c) Needle $45^{\circ}$ to the front.

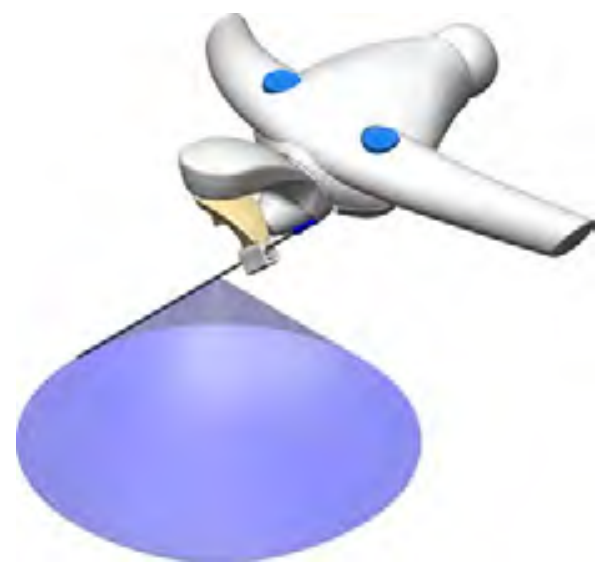

(b) Needle $45^{\circ}$ to the right.

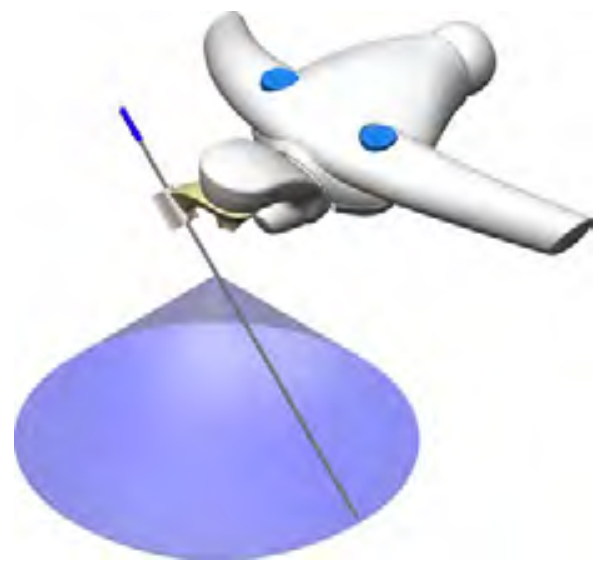

(d) Needle $45^{\circ}$ to the left.

Figure 3.7: Needle trajectories at four quadrants of the $90^{\circ}$ conical action range of the needle guide.

\section{X-ray transparency}

The OM and the adjoining part of the LM arm have been designed for sufficiently X-ray transparency to avoid unacceptable distortion of the images of the second CT scan, which are used for segmentation of the fiducial markers and confirmation of the target pre-planned on the images of the initial scan or specification of a new target. This is accomplished by dedicated material selection for the components in these bodies, which include plastics such as PEEK, POM, nylon and teflon, glass fibre and carbon reinforced epoxies, and ceramics such as borosilic- 


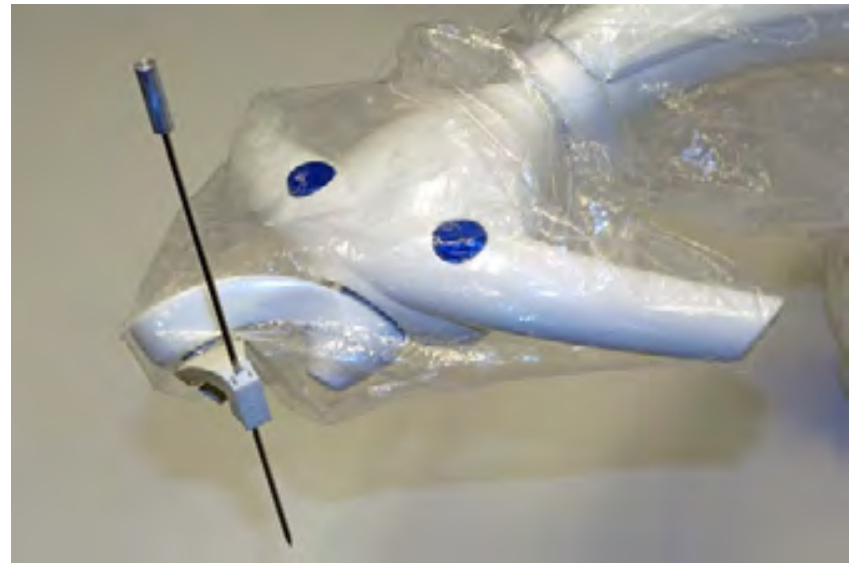

Figure 3.8: Disposable plastic cover placed over segment 1 , the main body of the $\mathrm{OM}$ and the LM arm to maintain a sterile field.

ate glass, aluminium oxide and silicon nitride. The 2-DOFs mechanism to aim the needle guide is remotely actuated via endless Dyneema cables on a pulley system by two electrical servomotors (Maxon [182] RE-max 24, $11 \mathrm{~W}$ ). Custom plastic fibre optic encoders are incorporated in the mechanism for precise motion control. Carbon nanotubes are used as an alternative for copper for electrical signal wiring of the push-buttons controlling the locking system. The servomotors and electronics for the encoders and locking system are placed in the lower part of the LM arm, outside the X-ray transparent volume.

\section{Sterility}

To maintain a sterile field, a disposable plastic cover is placed over segment 1 , the main body of the OM and the LM arm (Fig. 3.8). Segment 2 is connected to segment 1 through a hole in the cover and bolt locked. The aluminium oxide segment 2 and its PEEK bolt are sterilizable reusables. The selective laser sintered nylon needle clip and the RCM pointer are sterile disposables.

\subsubsection{Performance assessment}

To demonstrate the system's functionality and quantify its performance in terms of placement errors, the prototype was deployed for CT-guided placement of a 13 gauge $(\varnothing 2.4 \mathrm{~mm})$ steel needle with a cone tip in a static gelatin phantom, as an artificial substitute for the abdominal anatomy. The phantom consists of gelatin (15:100 gelatin powder to water weight ratio) cast in a plastic structural frame and covered by a synthetic chamois leather skin. Wooden $\varnothing 2 \mathrm{~mm}$ skewers were inserted in the structural frame and embedded in the gelatin. The tips of the 


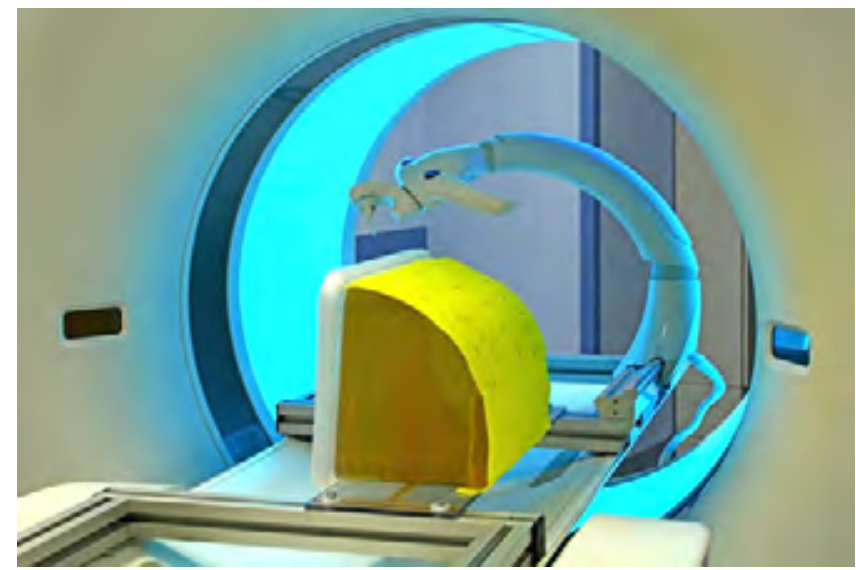

(a) Installation of the system and phantom on the CT scanner table via aluminium test frames.

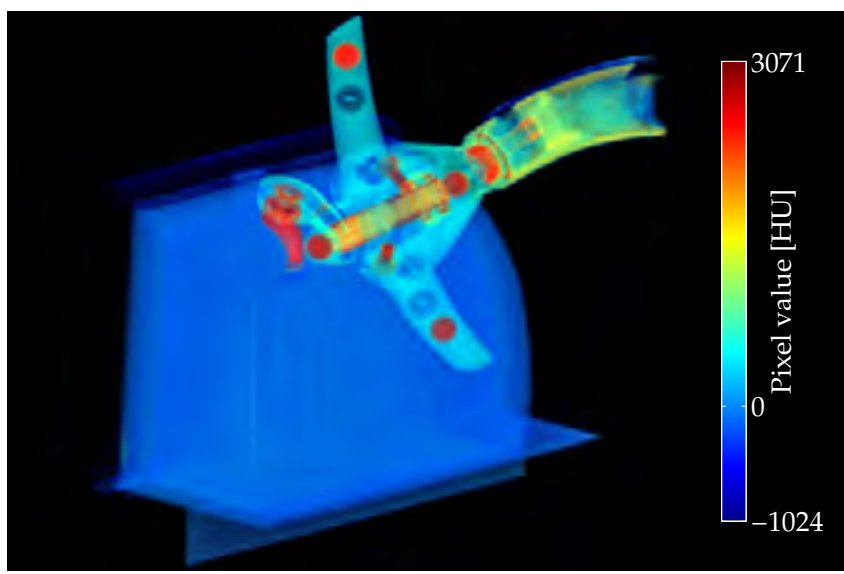

(b) First 3D CT image after manual placement of the OM and locking, used for OM-to-CT registration which yields the coordinates of entry point $\left(x_{E}\right)$, and for specification of the target coordinates $\left(x_{T}\right)$.

Figure 3.9: Performance assessment of the system for CT-guided needle placement in an abdominal phantom.

skewers served as targets. The position of the targets and corresponding entry points were predefined to prepare two sets of 12 paths, with approximate insertion depths of $80 \mathrm{~mm}$ and $160 \mathrm{~mm}$ respectively and covering a range of angles oblique to the transversal image plane. The entry points were marked on the skin prior to the experiment. A Siemens Somatom Force CT scanner was used for imaging. The rail of the system and the phantom were mounted on aluminium test frames fixed to the CT table (Fig. 3.9a). 


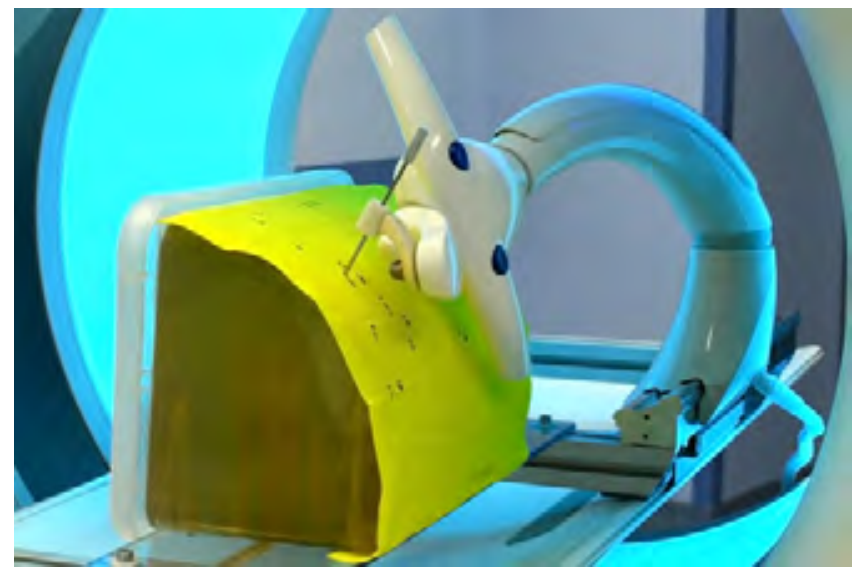

(c) Manually inserted needle after automatic aiming of the needle guide for physical guidance.

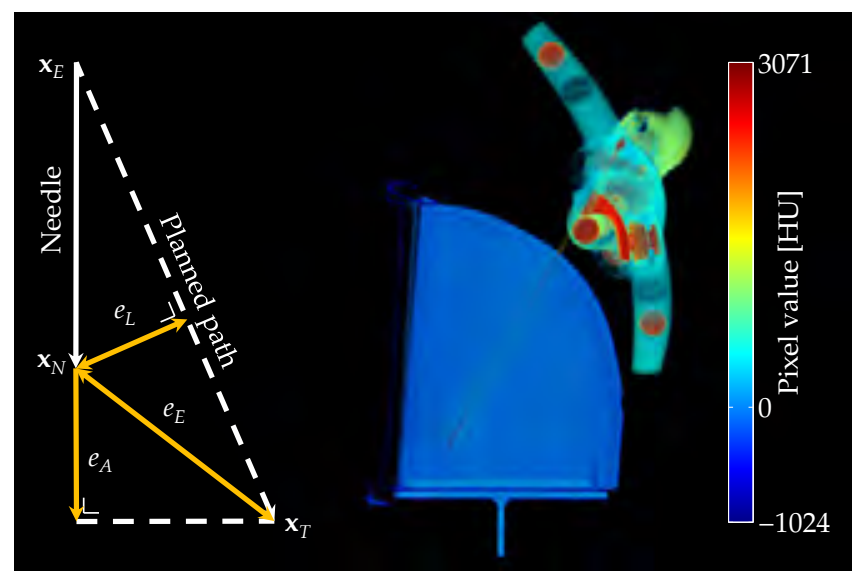

(d) Second 3D CT image after manual needle insertion to determine the needle tip position $\left(\boldsymbol{x}_{N}\right)$, and illustration of the geometrical definition of the Euclidean $\left(e_{E}\right)$, lateral $\left(e_{L}\right)$ and axial eror $\left(e_{A}\right)$.

Figure 3.9: Performance assessment of the system for CT-guided needle placement in an abdominal phantom - continued.

For each path, the OM was placed and locked such that the RCM pointer coincided with the marked entry point. A first 3D CT image was acquired, covering the OM and the phantom (Fig. 3.9b) for OM-to-CT registration, which yields the coordinates of entry point $\left(x_{E}\right)$, and for specification of the appropriate target coordinates $\left(x_{T}\right)$. The RCM pointer was removed and the needle guide was automatically aimed upon pressing the aim button. A small incision in the skin at the entry point was created, followed by insertion of the needle to specified 
Table 3.1: Results summary of the performance assessment. $M V=$ mean value, $\mathrm{SD}=$ standard deviation, $\mathrm{MN}=$ minimum value, $\mathrm{MX}=$ maximum value .

\begin{tabular}{lc|ccc|ccc}
\hline Parameter & Units & $\mathbf{M V} \pm \mathbf{S D}$ & $\mathbf{M N}$ & $\mathbf{M X}$ & $\mathbf{M V} \pm \mathbf{S D}$ & $\mathbf{M N}$ & $\mathbf{M X}$ \\
\hline \# Paths & {$[-]$} & 12 & & & 12 & & \\
Insertion depth & {$[\mathrm{mm}]$} & $79.0 \pm 8.4$ & 66.0 & 91.1 & $156.0 \pm 6.9$ & 144.4 & 166.9 \\
In-plane angle & $\left.{ }^{\circ}\right]$ & $30.7 \pm 35.9$ & -19.0 & 79.1 & $40.8 \pm 33.5$ & -8.6 & 86.6 \\
Out-of-plane angle & {$\left[^{\mathrm{o}}\right]$} & $21.8 \pm 19.5$ & 1.6 & 50.0 & $18.3 \pm 17.9$ & 1.8 & 41.5 \\
\hline Euclidean error & {$[\mathrm{mm}]$} & $1.2 \pm 0.6$ & 0.0 & 2.0 & $2.1 \pm 0.7$ & 1.0 & 3.1 \\
Lateral error & {$[\mathrm{mm}]$} & $1.0 \pm 0.5$ & 0.0 & 1.9 & $2.0 \pm 0.7$ & 0.7 & 3.1 \\
Axial error & {$[\mathrm{mm}]$} & $0.6 \pm 0.4$ & 0.0 & 1.4 & $0.7 \pm 0.4$ & 0.1 & 1.2 \\
\hline
\end{tabular}

depth (Fig. 3.10c). A second 3D CT image was acquired (Fig. 3.10d) to determine the coordinates of the needle tip $\left(x_{N}\right)$. Three errors are geometrically defined by the coordinates $x_{E}, x_{T}$ and $x_{N}$ : the Euclidean error (the point-to-point distance between needle tip and target):

$$
e_{E}=\left\|x_{N}-x_{T}\right\|,
$$

the lateral error (the maximum distance between needle tip and planned path, due to deviation in direction):

$$
e_{L}=\frac{\left\|\left(\boldsymbol{x}_{N}-\boldsymbol{x}_{E}\right) \times\left(\boldsymbol{x}_{N}-\boldsymbol{x}_{T}\right)\right\|}{\left\|\boldsymbol{x}_{T}-\boldsymbol{x}_{E}\right\|},
$$

and the axial error (the distance along the needle from tip to the point closest to the target, due to deviation in insertion depth):

$$
e_{A}=\left\|\frac{\left(x_{T}-x_{E}\right) \cdot\left(x_{N}-x_{E}\right)}{\left\|x_{N}-x_{E}\right\|}-\right\| x_{N}-x_{E}\|\| .
$$

Table 3.1 summarizes the results of the $2 \times 12$ punctures. The two sets of paths effectively had insertion depths of $79.0 \pm 8.4 \mathrm{~mm}$ and $156.0 \pm 6.9 \mathrm{~mm}$ respectively, with in-plane angles of $-19^{\circ}$ to $79.1^{\circ}$ and $-8.6^{\circ}$ to $86.6^{\circ}$, and out-of plane angles of $1.6^{\circ}$ to $50.0^{\circ}$ and $1.8^{\circ}$ to $41.5^{\circ}$. A Euclidean error of $1.2 \pm 0.6$ was achieved at $79.0 \pm 8.4 \mathrm{~mm}$ insertion depth, with a lateral error of $1.0 \pm 0.5$ and an axial error of $0.6 \pm 0.4$. For an approximately doubled insertion depth of $156.0 \pm 6.9 \mathrm{~mm}$, the Euclidean and lateral errors scale up to $2.1 \pm 0.7 \mathrm{~mm}$ and $2.0 \pm 0.7 \mathrm{~mm}$ respectively, and the axial error remains comparable at $0.7 \pm 0.4 \mathrm{~mm}$.

The image noise due to the presence of the system in the CT field of view was assessed as a preliminary validation of its CT compatibility. The same slice volume was hereto repeatedly CT scanned, first containing the phantom only 


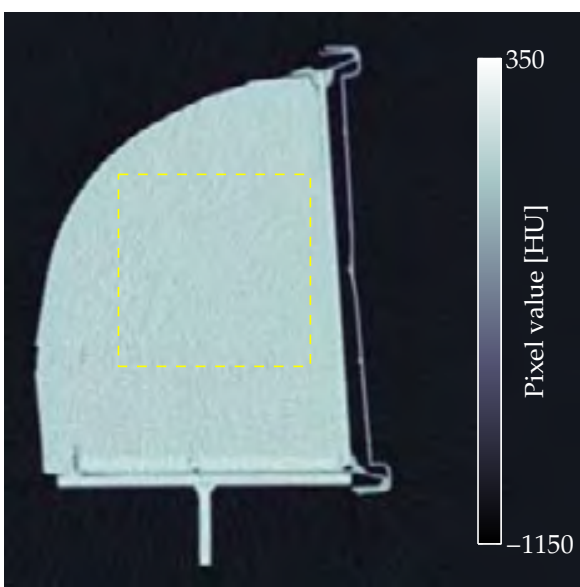

(a) Phantom-only image with outlined ROI. Window level/width: $-400 / 1500 \mathrm{HU}$.

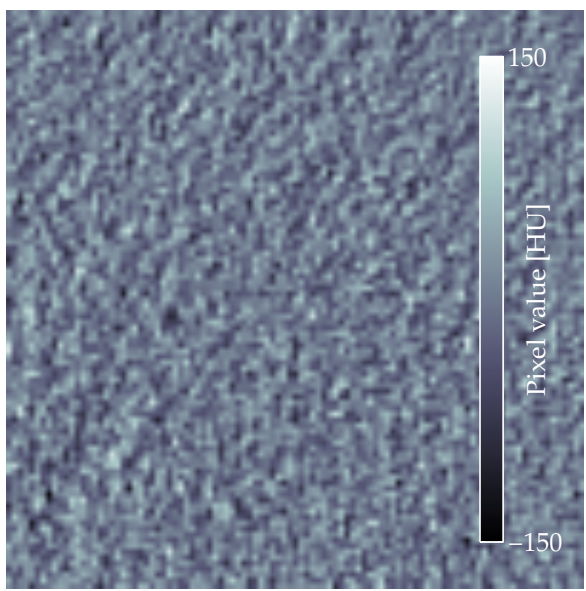

(c) ROI noise image of the phantom-only image. $\mathrm{MV} \pm \mathrm{SD}=0.0 \pm 31.4 \mathrm{HU}$.

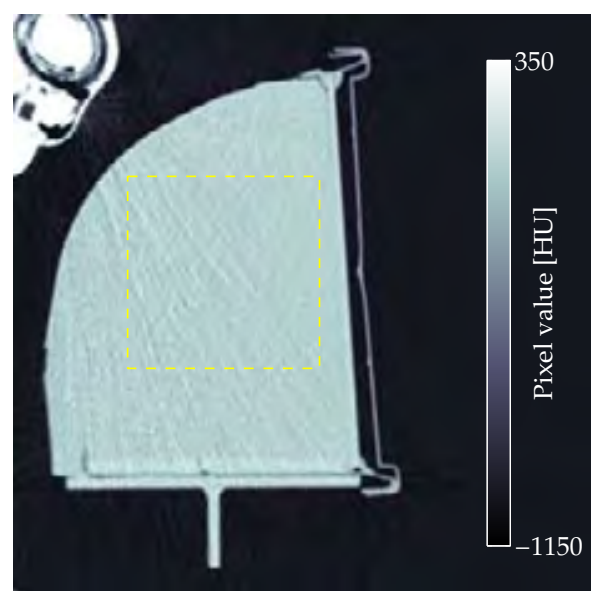

(b) Phantom-plus-system image with outlined ROI. Window level/width: $-400 / 1500 \mathrm{HU}$.

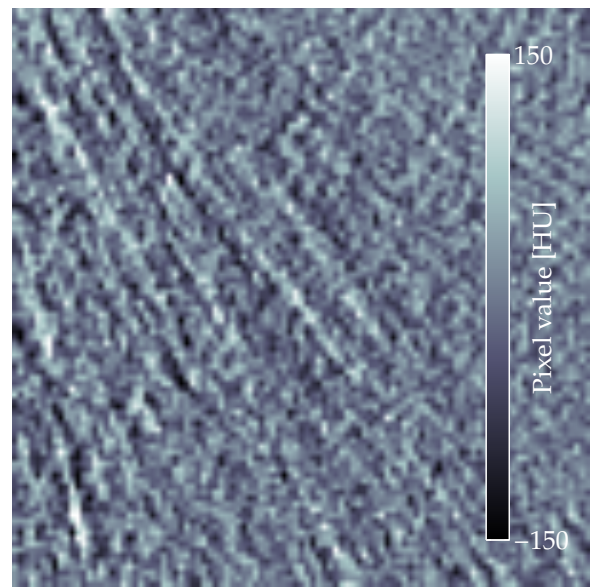

(d) ROI noise image of the phantom-plussystem image. $\mathrm{MV} \pm \mathrm{SD}=-0.3 \pm 42.9 \mathrm{HU}$.

Figure 3.10: CT image noise assessment.

(Fig. 3.10a) and subsequently the phantom plus system (Fig. 3.10b). The homogeneous gelatin in the phantom is expected to have a uniform image color or pixel value. A $100 \times 100$ pixel region of interest (ROI) was selected in the crosssection of the gelatin, with a mean pixel value of 33.9 HU. Subtraction of this value from the ROI of the phantom-only CT image yields the noise image shown in Fig. 3.10c, with an inherently zero mean value and a standard deviation of $31.4 \mathrm{HU}$ as a measure for the noise level. Subtraction of the same value of 33.9 
HU from the ROI of the phantom-plus-system CT image yields the noise image shown in Fig. 3.10d, with a mean value of $-0.3 \mathrm{HU}$ and an increased standard deviation of $\pm 42.9 \mathrm{HU}$. More importantly, patterning can be observed in the phantom-plus-system noise image Fig. 3.10d, known as streaking artifacts [183].

\subsection{Discussion}

A novel system for CT-guided percutaneous needle placement in the thorax and abdomen was presented, designed with a focus on clinically acceptability, wide applicability and usability. The system provides precise alignment of a needle guide for needle placement in a single insertion, based on a straight needle trajectory and performing imaging and needle insertion during a respiratory pause in the same respiratory phase to minimize deviation of the target position, as in current clinical practise.

The prime consideration towards clinical acceptability is to leave the simple yet responsible task of needle insertion in the hands of the physician, with inherent haptic feedback. Compliance to sterility requirements is achieved by application of a sterile cover and sterile reusables or disposables. A wide clinical applicability is created by the freedom of placement of the OM around the patient and the angular range of the RCM mechanism to aim the needle guide and its range of compatible needle diameters. This enables accessibility of most body sites via in- or out-of-plane paths and extends the potential applicability beyond the original use case of biopsy and ablation in the liver and lungs.

The usability of the system originates in selection of its functions based on a detailed analysis of the existing clinical workflow and freehand method. The novel workflow is largely identical except for the elimination of iterative steps, to reduce tissue damage and patient $X$-ray exposure and to improve the timeefficiency of the procedure. The challenging task of needle aiming is made userindependent. OM-to-CT registration and calculations of segment angles and insertion depth are handled by software, and locking and aiming are automated and push-button controlled. The GUI is envisioned to be integrated as a minor plug-in in the image viewing software of the CT scanner to take advantage of its advanced features and avoid the need for an external laptop. The substitutive user effort due to introduction of the system consists of its initial installation in the intervention suite, pre-procedural component sterilization and sterile cover application.

The functionality of the system to place needles in a single insertion was demonstrated by approaching 24 targets via in- and out-of-plane paths in a static gelatin phantom. The achieved Euclidean placement errors of $1.2 \pm 0.6$ and $2.1 \pm 0.7 \mathrm{~mm}$ at $79.0 \pm 8.4$ and $156.0 \pm 6.9 \mathrm{~mm}$ insertion depth respectively satisfy the $\leqslant 5 \mathrm{~mm}$ requirement. The image noise added by the presence of the system in the field of view was assessed for a homogeneous region of interest. The impact of the observed streaking artefacts on the diagnostic value of the images and the 
potential benefit of available noise reduction techniques should be assessed by radiologists in clinical practise. A first clinical trial is in preparation to validate the system's guided method in comparison to the conventional freehand method. 


\title{
CHAPTER 4
}

\section{Deflection of needles for percutaneous procedures in the liver by axially guided insertion in ex vivo porcine liver tissue}

\author{
Maarten M. Arnolli, Alex Rosendaal, Michel C.J. Franken, \\ Dannis M. Brouwer and Ivo A.M.J. Broeders \\ Submitted to the International Journal of Computer Assisted Radiology E Surgery, \\ Springer.
}

\begin{abstract}
Purpose: A strategy to improve CT-guided percutaneous needle placement in the liver is proposed, in which a novel system provides precise axial alignment of a needle guide to reach a target in a single manual insertion. Needle deflection is a contributor to a required $\leqslant 5 \mathrm{~mm}$ overall placement error. The objective of this study is to quantify needle deflection for a selection of needles used in current clinical practise for percutaneous procedures in the liver.
\end{abstract}

Methods: Deflections of bevel and Franseen tip needles were empirically quantified for axially guided manual insertion in porcine liver tissue. A repetition of insertions for each needle was first performed using random axial orientation and subsequently for identical axial orientation.

Results: Deflections of $9.2 \pm 2.4$ and $9.6 \pm 1.5 \mathrm{~mm}$ were measured for bevel tip needles and $2.0 \pm 0.6 \mathrm{~mm}$ for a Franseen tip needle at $120 \mathrm{~mm}$ insertion depth. Repeatabilities improved when changing from random to identical axial orientation, from $8.9 \pm 1.6$ to $2.7 \pm 3.2 \mathrm{~mm}$ and from $9.3 \pm 1.6$ to $0.7 \pm 0.5 \mathrm{~mm}$ for bevel tip needles, and from $1.2 \pm 0.8$ to $0.7 \pm 0.3 \mathrm{~mm}$ for a Franseen tip needle.

Conclusions: The deflections of the Franseen tip needles are sufficiently small for an overall placement error of $\leqslant 5 \mathrm{~mm}$ to be feasible. The deflections of the bevel tip needles are unacceptable, but compensation by adjustment of the alignment of the needle guide may be feasible, provided the axial orientation of the bevel is known. 


\subsection{Introduction}

Minimally invasive biopsy and ablation of liver tumours are performed via needles inserted percutaneously using image-guidance to reach the target tissue. A freehand method is conventionally employed for CT-guided needle placement, which typically requires multiple iterations of needle insertion and imaging before satisfactory placement is achieved. A strategy for improved CT-guided percutaneous needle placement is proposed, in which a novel system provides precise axial alignment of a needle guide with a user-specified target. The needle is subsequently inserted manually through the needle guide to reach the target in a single insertion, without the need for intermediate imaging. The strategy should enable a needle placement error of $\leqslant 5 \mathrm{~mm}$, based on clinical needs.

In assessment of the proposed strategy, three contributors to the overall needle placement error are identified. Firstly, the alignment errors of the needle guide with respect to the specified target coordinates, which define the functional requirements directing the elaboration of the architectural and detail design of the novel system. Secondly, displacement of the target tissue due to patient movement or respiration, which is effectively minimized in current clinical practise by patient stabilization using cushioning and a breath hold during image acquisition and needle insertion in the same respiratory phase. Thirdly, needle-tissue interaction, causing deflection of the needle tip and displacement of the target tissue. The focus of this study is the contribution of needle deflection to the overall placement error.

The general deflection behaviour of a needle is well-known to be affected by the shape of its tip. Axi-asymmetric bevel tip needles deflect in direction of the bevel, which is advantageously used in conventional freehand needle placement to adjust an off-target needle trajectory by altering the axial orientation. Axisymmetric centre tip needles show less deflection and lack an intrinsic preferred direction. A literature review in search of quantification of needle deflection shows several empirical studies on insertions to limited depth in the field of spinal and dental anaesthesia [184-187]. Needle deflection in soft tissue, to reach targets in the kidney, liver and prostate, has been analysed in detail to verify models of needle-tissue interaction [188,192-199]. These are intended to predict deflection and provide control inputs for systems with active needle steering to avoid deviation from the axial trajectory [189-191], or to create curved trajectories on purpose to avoid critical or impenetrable tissue [200-202].

Increasingly thin, bevel tip needles have been the prime subject of investigation because of favourable steering capabilities. The majority of needles investigated in [184-202] have a bevel tip and range from 30 to 20 ga (gauge, Ø0.3 to $0.9 \mathrm{~mm}$ ) in diameter. Biopsy and ablation needles to target liver tumours however typically range from 22 to $13 \mathrm{ga}(\varnothing 0.7$ to $2.4 \mathrm{~mm}$ ) and both bevel and centre tip needle tips are available, as follows from a product inventorization of manufacturers Medax [203], Carefusion [204], Angiodynamics [205], Covidien [206], Boston Scientific [207] and RF Medical [208]. Figure 4.1 shows a plot of needle 


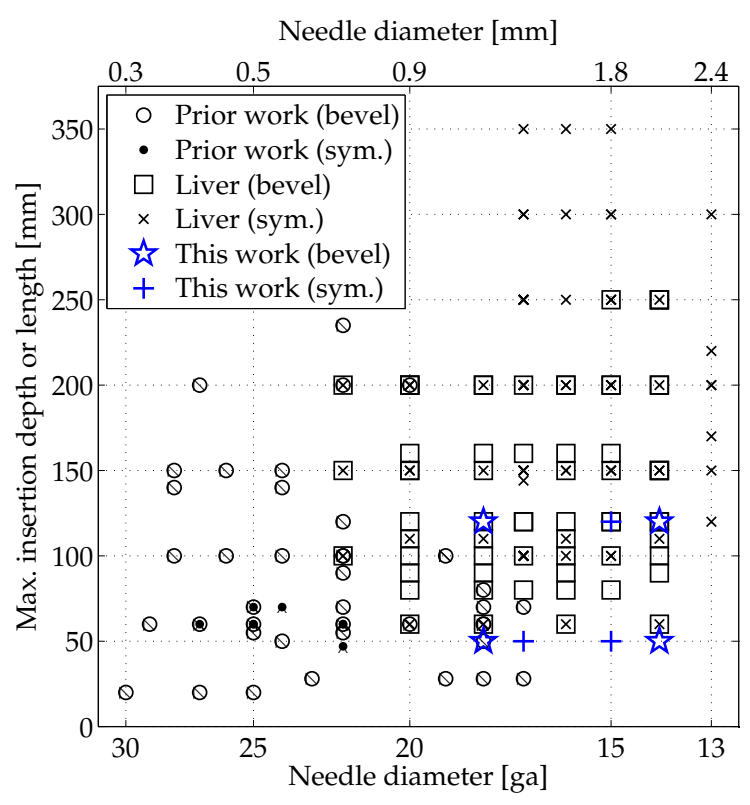

Figure 4.1: Needle diameter versus maximum insertion depth reported in prior work [184-196], diameter versus length of commercially available needles for procedures in the liver, and needle diameter versus maximum insertion depth investigated in this study.

diameter versus maximum insertion depth or needle length, illustrating a poor overlap of these needles with those investigated in the prior work [184-202].

The objective of this study is therefore to quantify needle deflection for guided manual insertion for a selection of needles used in current clinical practise for percutaneous procedures in the liver. Minimal deflection off the axial trajectory of the needle guide is desired for precise placement in a single insertion. A high repeatability is desired in case of larger deflection, to potentially compensate for deflection by adjusting the alignment of the needle guide before insertion. This article is structured into a section outlining the materials and methods used for the quantification, followed by the results and a discussion.

\subsection{Material \& methods}

An isolated experiment setup was designed to empirically quantify deflection for axially guided manual needle insertion in a soft tissue phantom (Fig. 4.2). In this section, the investigated needles, needle guides, phantom, measurement 


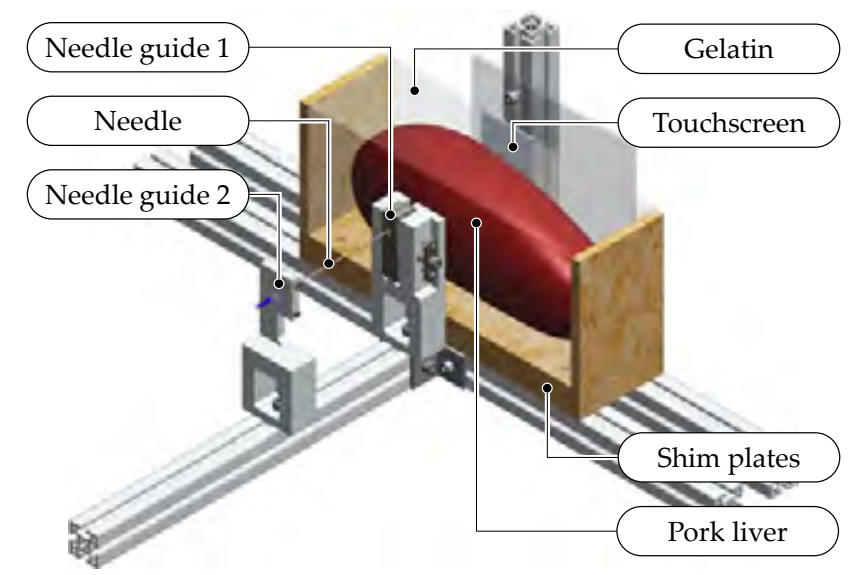

Figure 4.2: CAD model of the experiment setup with labelled components.

system, experiment execution and measurement interpretation are subsequently discussed.

\subsubsection{Needles}

Four needles used in current clinical practise in the liver were investigated:

- a 14 ga (Ø2.20 mm), 250 mm long, bevel tip Boston Scientific LeVeen standard needle electrode for RFA,

- an 18 ga (Ø1.15 mm), 200 mm long, bevel tip Medax universal biopsy needle,

- a 15 ga (Ø1.80 mm), 240 mm long, Franseen tip brachytherapy needle,

- a 17 ga (Ø1.45 mm), 155 mm long, Franseen tip Covidien Cool-tip RFA electrode.

The Boston Scientific LeVeen standard needle electrode for RFA has an open tip from which multiple tines can be deployed to increase the size of the ablation field (Fig. 4.3a and 4.3b). The Medax universal biopsy needle also has an open bevel tip and is intended for use with a biopsy gun, which shoots an inner tool and the outer needle subsequently to harvest a tissue sample (Fig. $4.3 \mathrm{c}$ and $4.3 \mathrm{~d}$ ). The Franseen tip needles have an axisymmetric, three-faceted shape and are closed (Fig. 4.3e and 4.3f). The brachterherapy needle is hollow for insertion of a radiation source. The Covidien Cool-tip RFA electrode incorporates an internal cooling water circuit beneficial to the ablation zone. 


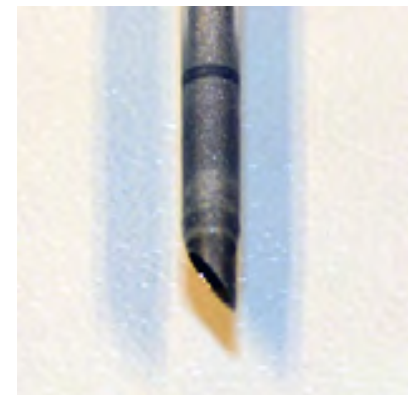

(a) Bevel tip of the 14 ga Boston Scientific LeVeen electrode.

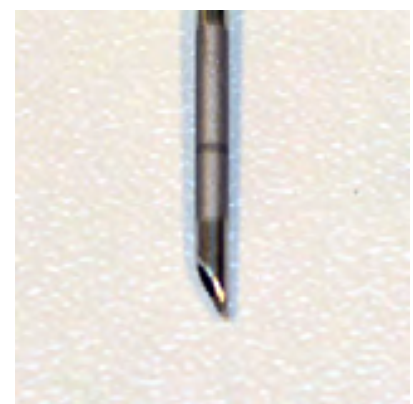

(c) Bevel tip of the 18 ga Medax biopsy needle.

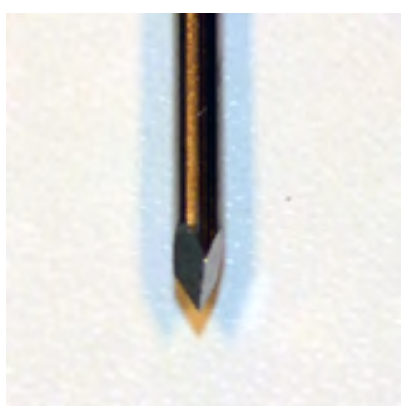

(e) Franseen tip of the 15 ga brachytherapy needle.

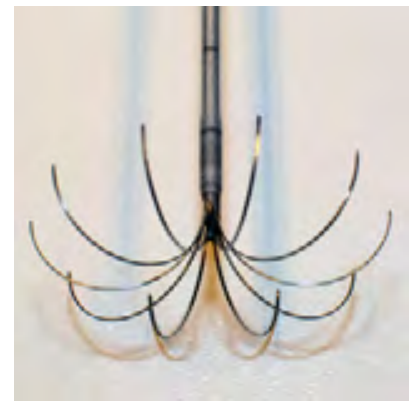

(b) Tip of the 14 ga Boston Scientific LeVeen electrode after deployment of its internal tines.

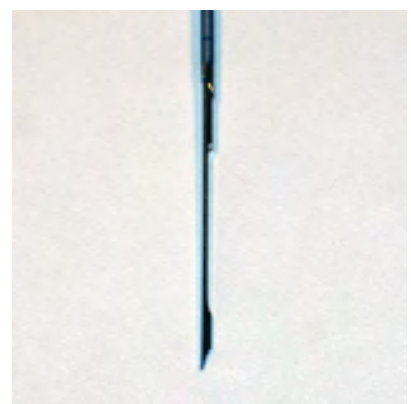

(d) Tip of the 18 ga Medax biopsy needle after deployment of its inner tool.

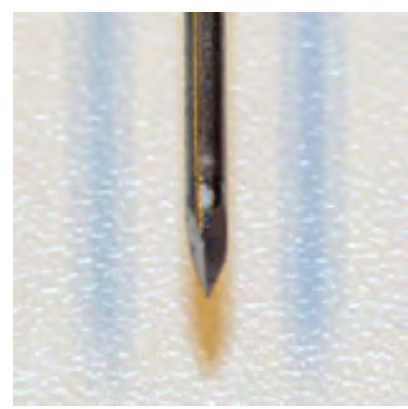

(f) Franseen tip of the $17 \mathrm{ga} \mathrm{Cov}$ idien Cool-tip RFA electrode.

Figure 4.3: Tips of the four investigated needles. 


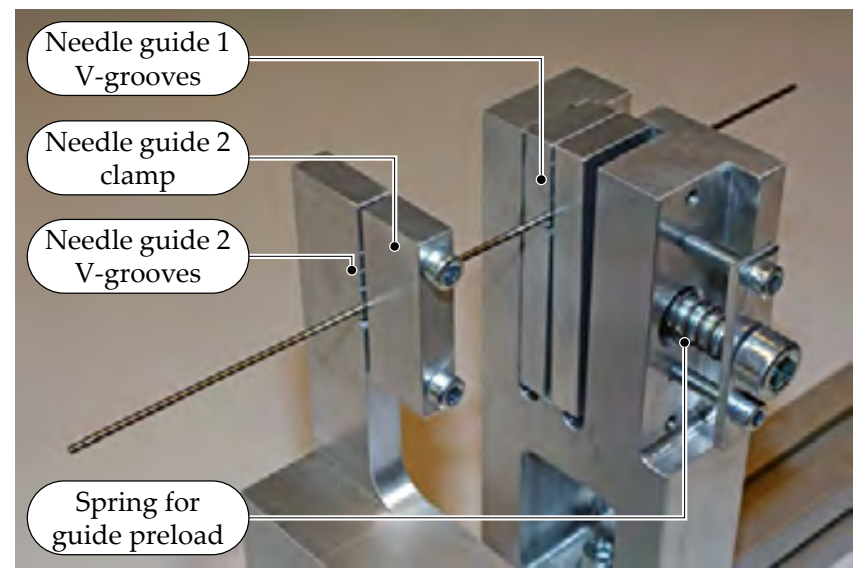

Figure 4.4: A needle held by the two needle guides of the experiment setup.

\subsubsection{Needle guides}

The experiment setup embodies a first axial needle guide of $25 \mathrm{~mm}$ length (Fig. 4.4). It constrains four degrees of freedom of the needle: two translations along- and two rotations about axes perpendicular to its longitudinal axis. Guidance with zero play is provided by V-grooves in an aluminium block, in which the needle is preloaded by a second aluminium block and a spring. The V-grooves have increasing depth to accommodate needle diameters from 22 to 11 ga (Ø0.7 to $3.2 \mathrm{~mm}$ ). The needle guide is mounted on a straight aluminium construction profile.

Maintaining identical axial orientation of the needle for subsequent insertions is expected to improve the repeatability of needle deflection, particularly of the bevel tip needles. A second needle guide is therefore installed to apply an optional constraint of the axial orientation of the needle by clamping its end (Fig. 4.4). It slides over the same aluminium construction profile in line with the first needle guide during insertion.

\subsubsection{Phantom}

Phantoms were created consisting of a porcine liver tissue embedded in gelatin and fortified at the bottom and sides by a wooden frame. The phantom is placed in front of the axial trajectory of the needle guide, at a distance of $35 \mathrm{~mm}$ to the first needle guide. To provide non-perforated tissue in front of the axial trajectory of the needle guide for each new insertion, the position of the phantom is horizontally and vertically adjustable, using a linear slide and adding shim plates respectively. Deflection of the needle tip is measured where it exits the back of the phantom. Phantoms of 50 and $120 \mathrm{~mm}$ thickness were created to investigate 


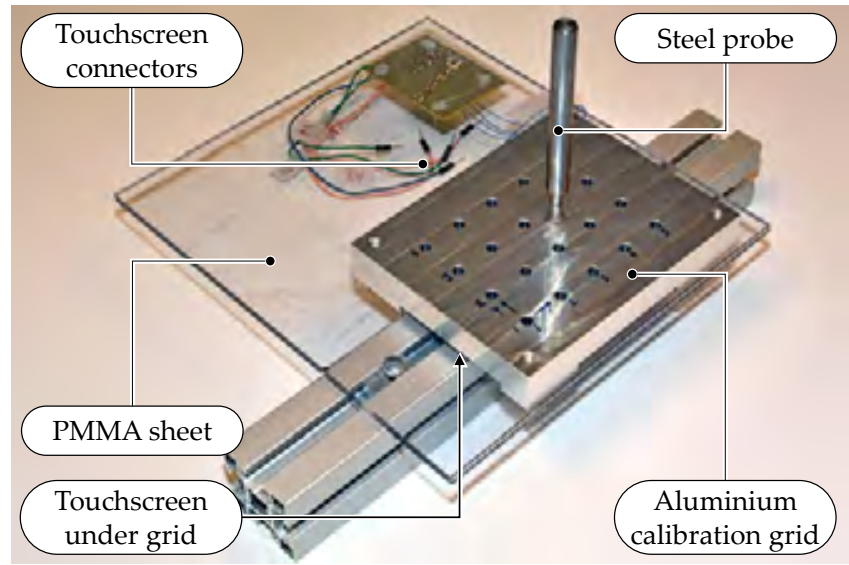

Figure 4.5: The touchscreen calibration setup.

two insertion depths.

\subsubsection{Measurement system}

The position measurement of the exiting needle tip was performed using a 89 by $68 \mathrm{~mm}$ resistive Nintendo DSi XL touchscreen placed $5 \mathrm{~mm}$ behind the phantom. Corresponding to the $x$ - and $y$-position of the touched positions on the screen are two analogue voltage outputs $U_{1}$ and $U_{2}$, which are digitalized and transferred to The MathWorks [209] Matlab, version R2007b, via an Arduino [210] UNO board with an Atmega 328P-PU micro-controller. The touchscreen was glued onto a PMMA sheet mounted on an aluminium construction profile, perpendicular to the direction of the needle guides, and such that the axial trajectory of the needle guides intersects with its centre.

The touchscreen was calibrated using an aluminium calibration grid mounted above the touchscreen, containing $5 \times 4=20$ holes in $x$ - and $y$-direction respectively at $20 \mathrm{~mm}$ apart (Fig. 4.5). Each hole has a positional error of $\leqslant 0.05 \mathrm{~mm}$ and a diameter of $5_{-0}^{+0.012} \mathrm{~mm}$, in which a steel probe with a centre tip and a diameter of $5_{-0.1}^{+0} \mathrm{~mm}$ can be inserted to hit the touchscreen and generate output signals corresponding to known relative positions. Fig. 4.6a shows a plot of the measured voltage outputs $U_{1}$ and $U_{2}$ at the calibration positions, which are averaged over 5 measurement sets of 20 calibration points each, 100 calibration points in total. A linear function is fitted through the mean set of calibration points for interpolation:

$$
X=A U+B,
$$

with $X=[x ; y]$ the coordinates of a touched position and $U=\left[U_{1} ; U_{2}\right]$ the corres- 


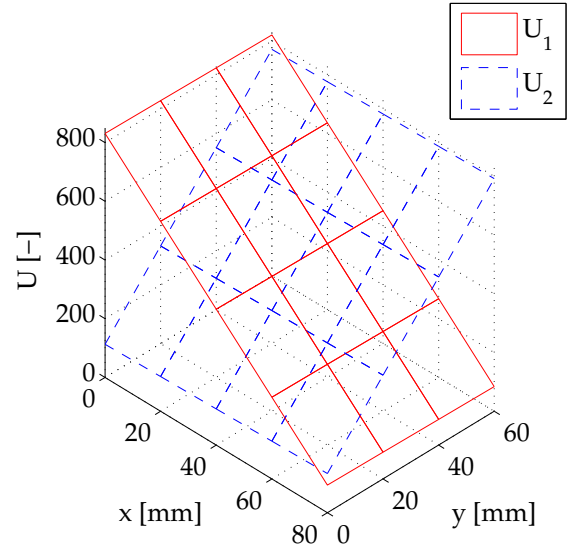

(a) Mean values of the two outputs of the touchscreen at the calibration points.

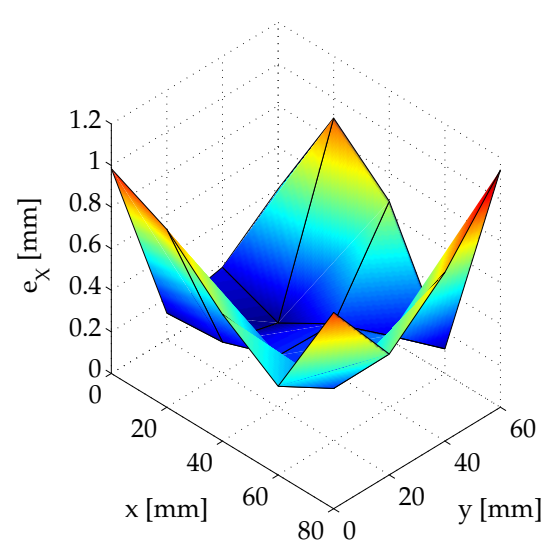

(b) Residual error $e_{X}$ after least squares linear fitting.

Figure 4.6: Calibration of the touchscreen for position measurement of the needle tip.

ponding voltage outputs. The coefficients $A$ and $\boldsymbol{B}$ of the linear fit are determined by minimization of the sum of the squared errors between the known calibration position and the linear fit at each calibration point. The residual error of the fit with respect to a calibration point at coordinates $\boldsymbol{X}_{\mathcal{c}}$ with outputs $\boldsymbol{U}_{c}$ is calculated as:

$$
e_{X}=\left\|A U_{c}+B-X_{c}\right\| .
$$

Figure $4.6 \mathrm{~b}$ shows a plot of the residual errors of the fit with respect to the mean value of 5 measured outputs at each calibration point. These errors are largest at the outer points of the calibration grid. Since the needle guide aligns with the centre of the touchscreen, the $3 \times 2=6$ inner points of the grid are most important. The calibration procedure was repeated one week after the first time to verify the output stability of the touchscreen over time. Table 4.1 lists the mean values, standard deviations and maximum values of the residual errors of both calibrations with respect to the least squares linear fit on the mean values of the first calibration. Error values based on all $5 \times 20=100$ calibration points are listed, as well as values based on the $5 \times 6=30$ inner calibration points only. A maximum error of $0.52 \mathrm{~mm}$ is found for the inner calibration points, which is deemed sufficiently low for the purpose of the experiment. 
Table 4.1: Mean values (MV), standard deviations (SD) and maximum values (MX) of residual errors $e_{X}$ of two calibration sessions with respect to the least squares linear fit of the mean values of the first calibration.

\begin{tabular}{lccc}
\hline Calibration & \# Included points & $\begin{array}{c}\mathbf{M V} \pm \mathbf{S D} \\
{[\mathrm{mm}]}\end{array}$ & $\begin{array}{c}\mathbf{M X} \\
{[\mathrm{mm}]}\end{array}$ \\
\hline $1^{\text {st }}$ & 100 (all points) & $0.51 \pm 0.33$ & 1.24 \\
$2^{\text {nd }}$ & 100 (all points) & $0.55 \pm 0.33$ & 1.22 \\
$1^{\text {st }}$ & 30 (inner points) & $0.23 \pm 0.09$ & 0.39 \\
$2^{\text {nd }}$ & 30 (inner points) & $0.27 \pm 0.14$ & 0.52 \\
\hline
\end{tabular}

\subsubsection{Experiment execution}

Four needles, two insertion depths and random versus identical axial orientation for subsequent insertions yield $4 \times 2 \times 2=16$ differentiated experiments. This was limited to 14, due to the insufficient length of the 17 ga Franseen tip needle to perforate the $120 \mathrm{~mm}$ width of the phantom behind the needle guide.

At the start of each experiment, the phantom was removed and the needle was inserted through the needle guide to hit the touchscreen and measure the position of the tip of the undeflected needle. This homing measurement was repeated 5 to 7 times at the start of each of the 14 experiments. The phantom was then reinstalled and the needle was manually inserted between 15 and 22 times through the needle guide and phantom to hit the touchscreen and measure the tip position. In-between two needle insertions, the position of the phantom was shifted to provide non-perforated tissue in front of the axial trajectory of the needle guide for each new insertion (Fig. 4.7). In case no constraint on the axial orientation was applied, the needle was randomly axially rotated before each insertion.

\subsubsection{Measurement interpretation}

Variables are defined here for measurement interpretation. For each of the 14 experiments, $\bar{x}_{0}$ is the mean position of the homing measurements. For each needle inserted through the phantom, $x_{i}$ is the tip position measured by the touchscreen, where subscript $i$ labels the number of insertion. The mean tip position of an experiment consisting of $n$ insertions is denoted by $\bar{x}$ :

$$
\bar{x}=\frac{1}{n} \sum_{i=1}^{n} x_{i}-\bar{x}_{0} .
$$




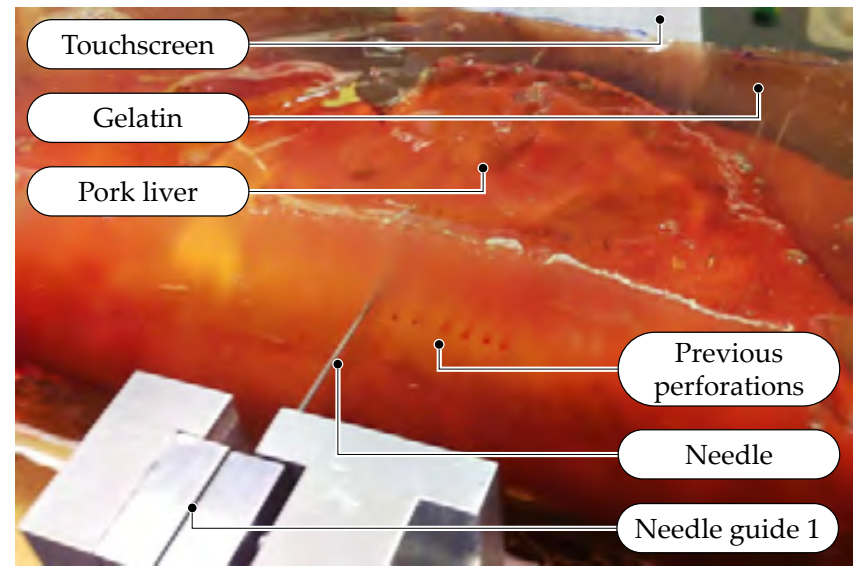

Figure 4.7: Needle insertion into the phantom.

The absolute deflection of the needle tip off the mean home position is denoted by $R$ :

$$
R_{i}=\left\|x_{i}-\bar{x}_{0}\right\| .
$$

The repeatability is defined as the distance of a deflected needle tip to the mean of $n$ deflected needle tip measurements, and is denoted by $r$ :

$$
r_{i}=\left\|x_{i}-\bar{x}\right\| .
$$

A large value for $R$ indicates large absolute deflection. A large value for $r$ indicates a poor repeatability of the deflection, either due to variability in absolute deflection or direction of the deflection.

\subsection{Results}

The mean values and standard deviations of the measured absolute deflections $R$ for random axial orientation are visualized by a bar diagram in Fig. 4.8a. The absolute deflection is relatively large for the 14 and 18 ga bevel tip needles: $4.1 \pm 2.2$ and $2.7 \pm 0.6 \mathrm{~mm}$ at $50 \mathrm{~mm}$, and $9.2 \pm 2.4$ and $9.6 \pm 1.5 \mathrm{~mm}$ at $120 \mathrm{~mm}$ insertion depth respectively. The deflection of the 15 and 17 ga Franseen tip needles is minimal: $1.7 \pm 0.3$ and $0.7 \pm 0.4 \mathrm{~mm}$ at $50 \mathrm{~mm}$ respectively, and $2.0 \pm 0.6 \mathrm{~mm}$ at 120 $\mathrm{mm}$ (15 ga only).

Slightly less absolute deflection of the 15 ga Franseen tip needle was measured when maintaining identical axial orientation for subsequent insertions, dropping from $2.0 \pm 0.6$ to $0.7 \pm 0.3 \mathrm{~mm}$ at $120 \mathrm{~mm}$ (Fig. $4.8 \mathrm{~b}$ ). The absolute deflections of the 


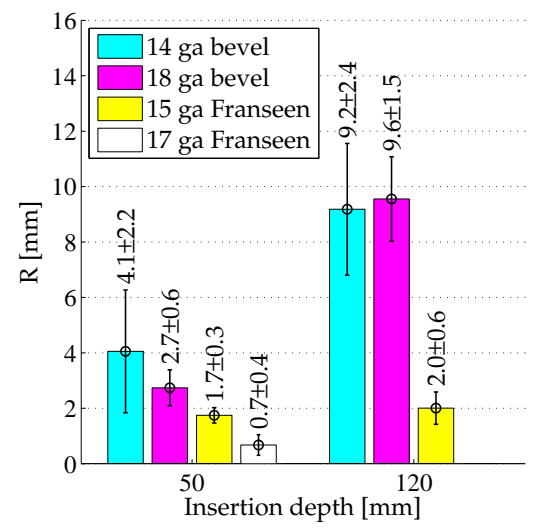

(a) Random axial orientation for each insertion.

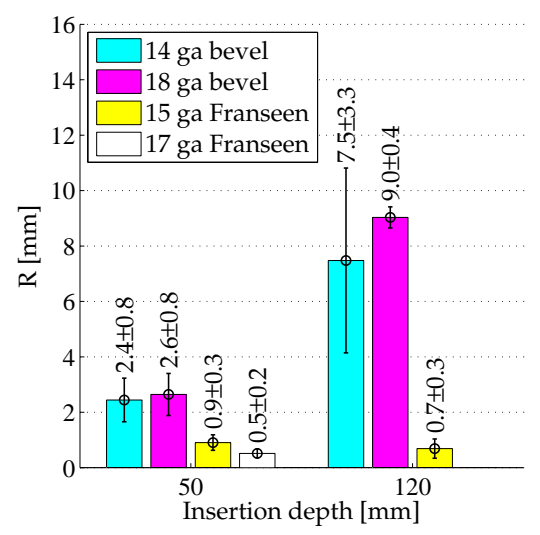

(b) Constraint applied for identical axial orientation for each insertion.

Figure 4.8: Measured absolute deflections.

two bevel tip needles remain significantly larger, $7.5 \pm 3.3$ and $9.0 \pm 0.4 \mathrm{~mm}$ at 120 $\mathrm{mm}$ for the 14 and 18 ga needle respectively.

More importantly, maintaining identical axial orientation significantly improves the repeatability of the bevel tip needles due to their preferred direction of deflection (Fig. 4.9a versus Fig. 4.9b). The improvement is particularly clear for the repeatability of the 18 ga bevel tip needle, which drops from $2.6 \pm 0.5$ to $0.8 \pm 0.6 \mathrm{~mm}$ at $50 \mathrm{~mm}$, and from $9.3 \pm 1.6$ to $0.7 \pm 0.5 \mathrm{~mm}$ at $120 \mathrm{~mm}$. These values are of the same order of magnitude of both Franseen tip needles. The repeatability of the 14 ga bevel tip needle drops from $3.2 \pm 1.1$ to $1.1 \pm 0.3 \mathrm{~mm}$ at $50 \mathrm{~mm}$, and from $8.9 \pm 1.6$ to $2.7 \pm 3.2 \mathrm{~mm}$ at $120 \mathrm{~mm}$. The deviation off the mean value at 120 $\mathrm{mm}$ is still large compared to the 18 ga bevel and 15 ga Franseen tip needles.

The results of the 6 experiments for $120 \mathrm{~mm}$ insertion depth are illustrated by polar plots in Fig. 4.10. A circular distribution of tip positions around the centre is visible for the two bevel tip needles for random axial orientation (Fig. 4.10a and Fig. 4.10c). The tip positions gather towards the preferred direction of deflection when maintaining identical axial orientation, for the 14 ga needle (Fig. 4.10b) and particularly the 18 ga needle (Fig. 4.10d). The tip positions of the 15 ga Franseen tip needle are closely grouped around the centre for both random and identical axial orientation (Fig. 4.11e and Fig. 4.11f). 


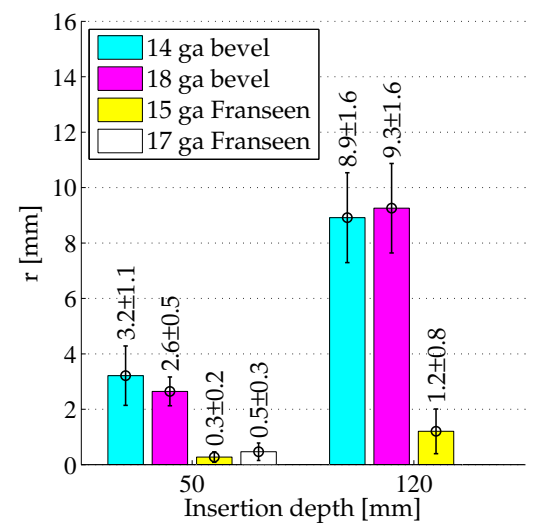

(a) Random axial orientation for each insertion.

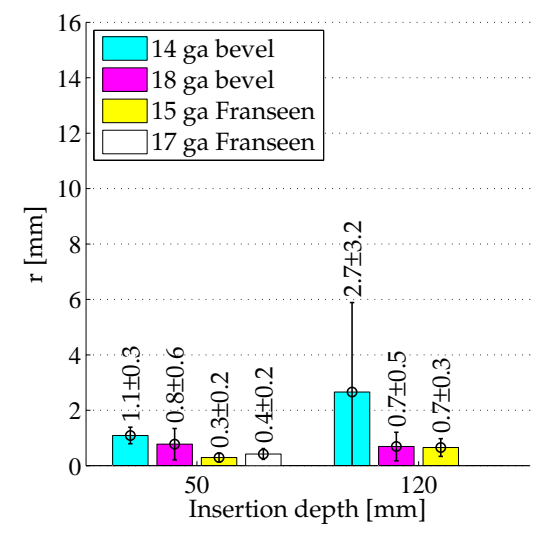

(b) Constraint applied for identical axial orientation for each insertion.

Figure 4.9: Measured repeatabilities.

\subsection{Discussion}

The deflections of four needles used in current clinical practise for percutaneous procedures in the liver have been quantified for insertion in porcine liver tissue. The quantification is part of an assessment of a proposed strategy to improve CTguided needle placement, in which a system provides precise axial alignment of a needle guide with a user-specified target for physical support during manual needle insertion. Needle deflection is a contributor to the required $\leqslant 5 \mathrm{~mm}$ overall placement error.

The $2.7 \pm 0.6 \mathrm{~mm}$ deflection at a depth of $50 \mathrm{~mm}$ of the 18 ga bevel tip biopsy needle is of the same order of magnitude as the reported 2.4 to $3.4 \mathrm{~mm}$ deflection of a hollow bevel tip needle in gelatin, beef muscle and pig heart in [190,191]. Compared to the 9.2 and $9.6 \mathrm{~mm}$ mean deflections of the investigated 14 and 18 ga bevel tip needles at $120 \mathrm{~mm}$, larger deflections of thinner bevel tip needles (28 ga to 18 ga) are reported in $[192,193,200]$ at the same depth, ranging from 13 to 46 $\mathrm{mm}$ in different gel phantoms. In accordance with the measured deflections of the 17 and 15 ga Franseen tip needles, reported deflections of thinner axisymmetric tip needles (27 to $22 \mathrm{ga}$ ) are minimal: less than $1.5 \mathrm{~mm}$ up to $70 \mathrm{~mm}$ insertion depth [184-186].

The measured deflections of the two axisymmetric Franseen tip needles up to $120 \mathrm{~mm}$ are sufficiently small for an overall placement error of $\leqslant 5 \mathrm{~mm}$ to be feasible, independent of the axial orientation of the needles. Deflections of the two bevel tip needles consume most of the $\leqslant 5 \mathrm{~mm}$ error budget at a depth of 50 $\mathrm{mm}$ insertion depth, and unacceptably exceed it at $120 \mathrm{~mm}$ depth. The proposed strategy is therefore primarily suited for the placement of needles with axisym- 


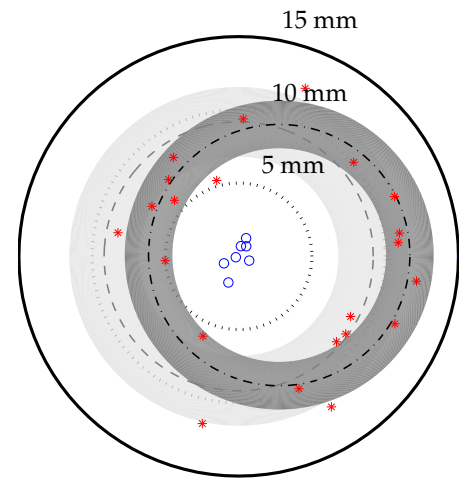

(a) 14 ga bevel tip needle, random axial orientation for each insertion: $R=9.2 \pm 2.4$ $\mathrm{mm}, r=8.9 \pm 1.6 \mathrm{~mm}$.

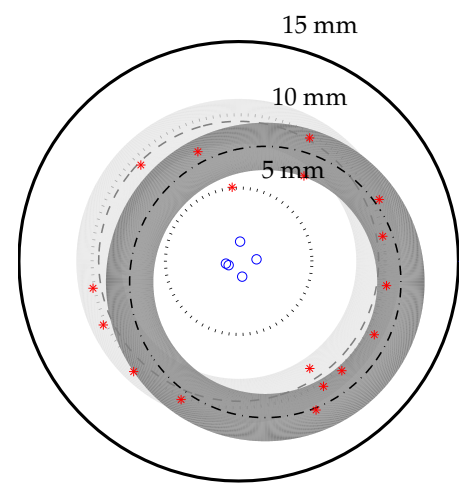

(c) 18 ga bevel tip needle, random axial orientation for each insertion: $R=9.6 \pm 1.5$ $\mathrm{mm}, r=9.3 \pm 1.6 \mathrm{~mm}$.

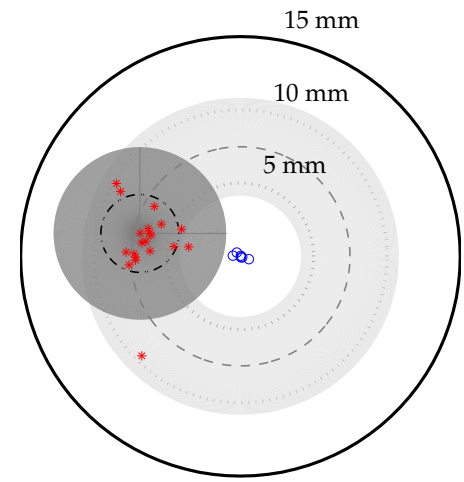

(b) 14 ga bevel tip needle, constraint applied for identical axial orientation for each insertion: $R=7.5 \pm 3.3 \mathrm{~mm}, r=2.7 \pm 3.2 \mathrm{~mm}$.

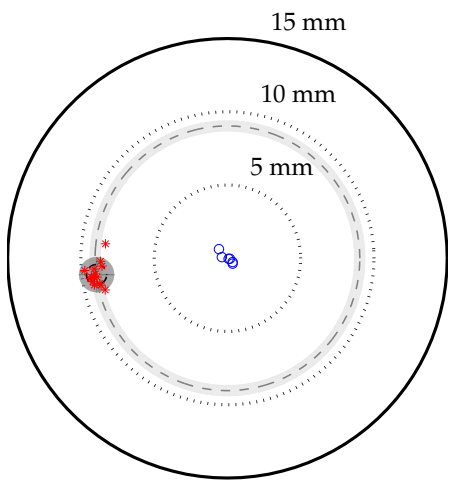

(d) 18 ga bevel tip needle, constraint applied for identical axial orientation for each insertion: $R=9.0 \pm 0.4 \mathrm{~mm}, r=0.7 \pm 0.5 \mathrm{~mm}$.

Figure 4.10: Polar plots of the results of 6 experiments at $120 \mathrm{~mm}$ insertion depth, with radius grids at 5,10 and $15 \mathrm{~mm}$. The centre of each plot coincides with $\bar{x}_{0}$, the mean of 6 homing measurements, which are represented by the small circles (०). Asterisks $(*)$ represent measured tip positions $x_{i}$. The light gray dashed line circle (--) and band visualize the mean value and standard deviation of absolute deflection $R$ off mean home position $\bar{x}_{0}$. The dark gray dashed line circle $(-\cdot-)$ and band visualize the mean value and standard deviation of repeatability $r$ around mean deflection $\bar{x}$. 


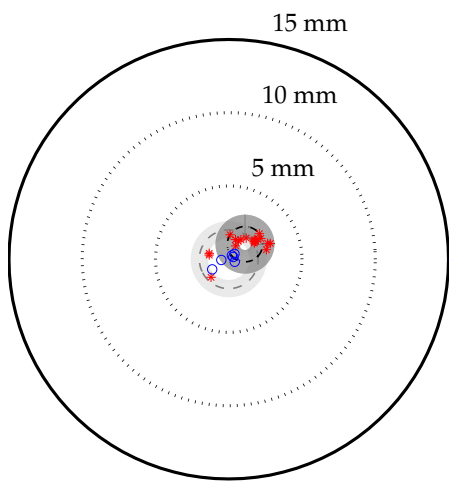

(e) 15 ga Franseen tip needle, random axial orientation for each insertion: $R=2.0 \pm 0.6$ $\mathrm{mm}, r=1.2 \pm 0.8 \mathrm{~mm}$.

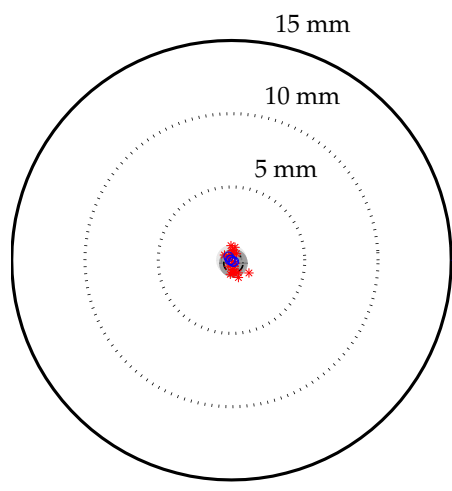

(f) 15 ga Franseen tip needle, constraint applied for identical axial orientation for each insertion: $R=0.7 \pm 0.3 \mathrm{~mm}, r=0.7 \pm 0.3$ $\mathrm{mm}$.

Figure 4.10: Polar plots of the results of 6 experiments at $120 \mathrm{~mm}$ insertion depth - continued.

metric tip.

For precise placement of bevel tip needles in a single insertion, compensation for deflection by adjustment of the alignment of the needle guide before insertion provides a potential solution. This demands a high repeatability of deflection with respect to the needle guide, which largely depends on the axial orientation of the needle. A repeatability of the same order of magnitude as the Franseen tip needles was found for the 18 ga bevel tip needle for insertions at identical axial orientation. Compensation to meet the requirement is therefore feasible if this needle is inserted through the needle guide at a known and constant axial orientation. The repeatability of the 14 ga bevel tip needle improves for identical axial orientation, but remains insufficient to confidently meet the $\leqslant 5 \mathrm{~mm}$ requirement by compensation.

The presented results apply to needle insertions in porcine liver tissue embedded in gelatin. It provides a more realistic phantom than homogeneous gel or foam, but is nonetheless an isolated and simplified substitute for the complete human anatomy. The surrounded suspension of the liver in the body and transitions of fat and muscle tissue layers may have additional effects on needle deflection, which should be addressed in future in vivo studies. 


\title{
CHAPTER 5
}

\section{A precision system for CT-guided needle placement in the thorax and abdomen - design \& performance analysis}

\author{
Maarten M. Arnolli, Martijn Buijze, Michel C.J. Franken, \\ Ivo A.M.J. Broeders and Dannis M. Brouwer \\ Submitted to the Journal of Medical Devices, American Society of Mechanical \\ Engineers.
}

\begin{abstract}
Background A system was developed for CT-guided needle placement in the thorax and abdomen, providing precise aiming of a needle guide to reach a user-specified target in a single manual insertion. The system's technical design is presented and its performance in terms of placement error in air is analysed.
\end{abstract}

Methods The individual contributions to the placement error of a fiducial marker based system-to-CT registration system, a 2-DOFs drive system to aim the needle guide, and a structural link between needle guide and CT table were experimentally determined, in addition to the placement error of the overall system.

Results A contribution of $0.81 \pm 0.34$ was determined for the registration system, $<1.2 \mathrm{~mm}$ and $<3.3 \mathrm{~mm}$ for the drive system, and $0.35 \mathrm{~mm}$ and $0.43 \mathrm{~mm}$ for two load cases of the structural link. The overall unloaded system achieved $1.0 \pm 0.25$ and $2.6 \pm 0.7 \mathrm{~mm}$ at 100 and $250 \mathrm{~mm}$ depth respectively.

Conclusions The overall placement errors in air do not exceed the $\leqslant 5 \mathrm{~mm}$ error specified as a clinical user requirement for needle placement in tissue. 


\subsection{Introduction}

Minimally invasive biopsy (tissue sampling for diagnostic pathology) and ablation (tissue destruction) of liver and lung tumour tissue are typically performed via a needle, which is percutaneously (through the skin) inserted to reach the target tissue using image-guidance, including $X$-ray computed tomography (CT) $[3,4,13,48]$. A freehand method is conventionally applied for needle placement, which has a user-dependent performance and generally requires multiple iterations of insertion and CT scanning for evaluation to achieve satisfactory placement. Various systems have been developed to improve this method, from simple passive aids to fully automated robots [72]. Examples of systems that provide (semi-)automated needle guidance are the patient mounted Robopsy [140], the table mounted Acubot [159], INNOMOTION [162] and iSYS [151] Medizintechnik iSYS1, and the floor mounted Perfint [169] ROBIO and MAXIO. Despite the various attempted strategies and often technically sound solutions, widespread clinical adoption of any system has yet to be achieved.

A novel table mounted system for CT-guided percutaneous needle placement in the thorax and abdomen has been developed with a focus on clinical acceptability, applicability and usability (Fig. 5.1). The system provides automated aiming of a needle guide to reach a user-specified target in a single manual insertion. The system consists of an orientation module (OM) and a locking module (LM). The OM contains an automated two degrees of freedom (2-DOFs) mechanism consisting of two serially stacked rotatable segments to aim a needle guide about a remote centre of motion (RCM). The LM is a passive 6-DOFs linkage connecting the OM to the CT table and consists of a 3-DOFs ball joint 2, the LM arm, a 2-DOFs ball joint 1, the LM foot, and a 1-DOFs slider over a rail mounted on the CT table. The LM allows the OM to be manually placed around the patient lying on the CT table, such that the RCM coincides with the desired entry point for the needle on the patient's skin. The RCM is hereto indicated by a removable pointer in the needle guide. The OM is then locked with respect to the CT table by push-button controlled simultaneous fixation of the joints of the LM. The volume containing the relevant anatomical part of the patient and the OM is CT scanned, acquiring a set of parallel images. The OM incorporates radiopaque spheres referred to as fiducial markers, which are located by segmentation of the CT images for registration of the OM coordinate system with respect to the CT coordinate system. The images are presented by a graphical user-interface (GUI) which allows for review of the anatomy and specification of the target coordinates for the needle tip. The required angles of the 2-DOFs mechanism to align the needle guide with the trajectory from RCM to target are automatically calculated and set by an internal drive system. The needle is inserted manually under physical guidance of the needle guide to calculated depth, measured using linear markings on the needle. A final CT scan is performed for placement evaluation before proceeding to biopsy or ablation.

The system's method invokes a technical requirement for the OM and the up- 


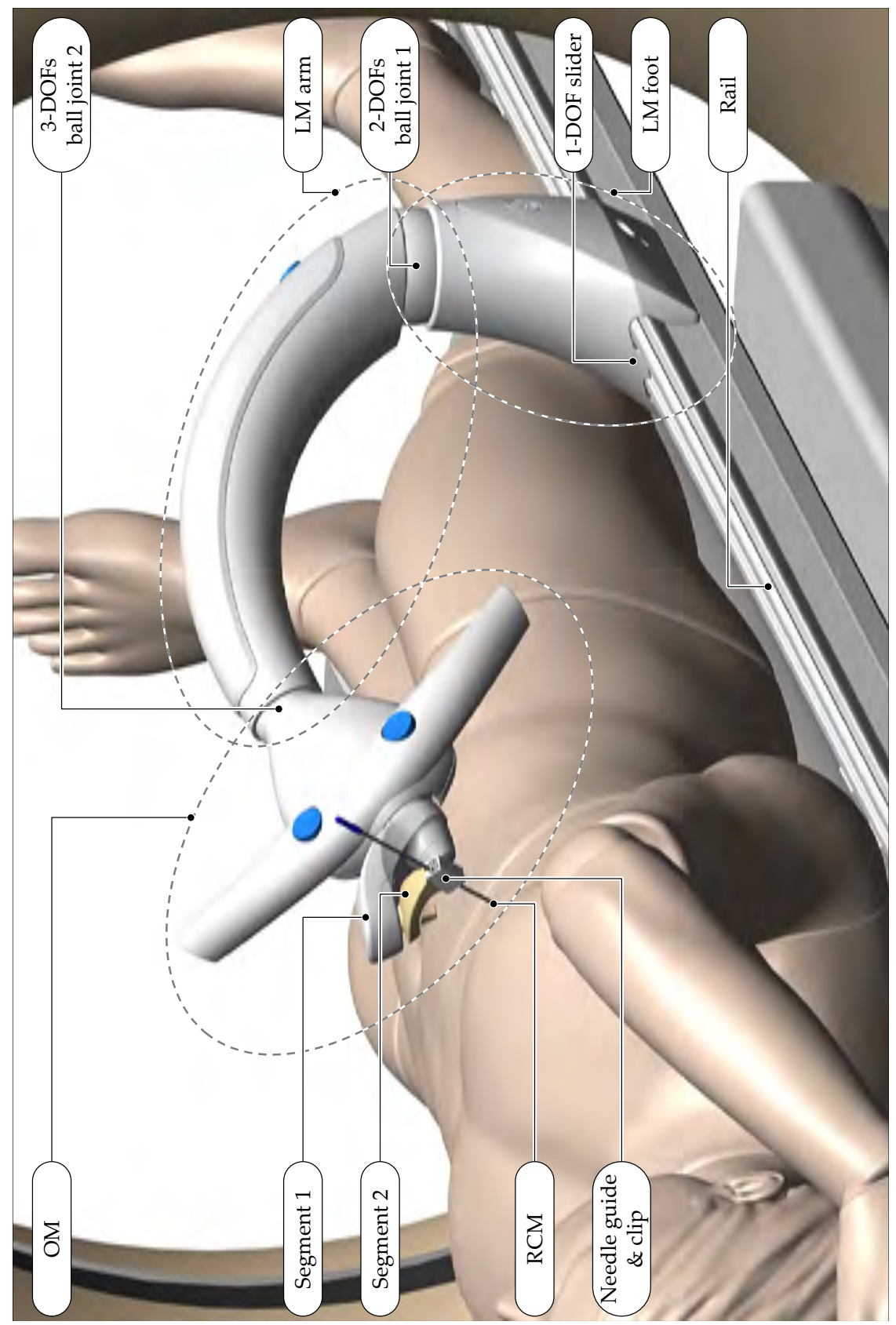

Figure 5.1: Overview of the system with labelled components. 
per part of the LM arm to be sufficiently X-ray transparent to avoid unacceptable deterioration of the diagnostic quality of the CT images. X-ray transparency depends on a material's atomic number and density, the objects thickness and ray energy [211]. As a practical guideline in the design of the novel system, construction materials containing elements beyond the third row of the periodic table were disqualified, such as (alloys of) titanium, iron and copper and some ceramics (zirconium oxide and tungsten carbide). The palette of remaining options includes rubbers, plastics (PA, PFTE, POM and PEEK), woods, (glass, aramid and carbon) fibre reinforced epoxies, aluminium and some ceramics (glass, aluminium oxide and silicon nitride). A clinical user requirement has furthermore been specified of $\leqslant 5 \mathrm{~mm}$ for the needle tip placement error in tissue, up to an extreme target depth of $250 \mathrm{~mm}$.

In this article, the technical design of the system is presented and its performance in terms of needle placement error for targets in air is analysed. The individual contributions of three prime subsystems to the overall placement error are determined: (1) target registration errors of the fiducial marker based OM-toCT registration system, (2) transmission errors of the drive system of the 2-DOFs mechanism to aim the needle guide, and (3) deflection of the needle guide due to deformation of the structural link with the CT table under external loads. This article contains a dedicated section for each of the three subsystems, divided into a materials and methods subsection in which the design of a subsystem is presented and the experiment setup to quantify its error contribution is described, followed by a results subsection. In a fourth section, the placement error of the overall system is quantified. The article concludes with a combined discussion.

\subsection{The registration system}

The OM incorporates four fiducial markers (Fig. 5.2) as part of the registration system to determine the position and orientation of the system's OM coordinate system with respect to the CT coordinate system. In this section, the design and method of the registration system are presented and its contribution to the placement error is quantified.

\subsubsection{Materials \& methods}

The four fiducial markers are Ø20 mm spheres of aluminium oxide, which yields a high image pixel value without significant distortion [181]. The 3D symmetry of each sphere yields circular cross-sections in a set of parallel images independently of its orientation, and thus independently of the orientation of the OM. The centre coordinates of each fiducial marker provide a point reference to the OM coordinate system with respect to the CT coordinate system. A minimum of three fiducial markers is required to fully define the transformation between OM and 


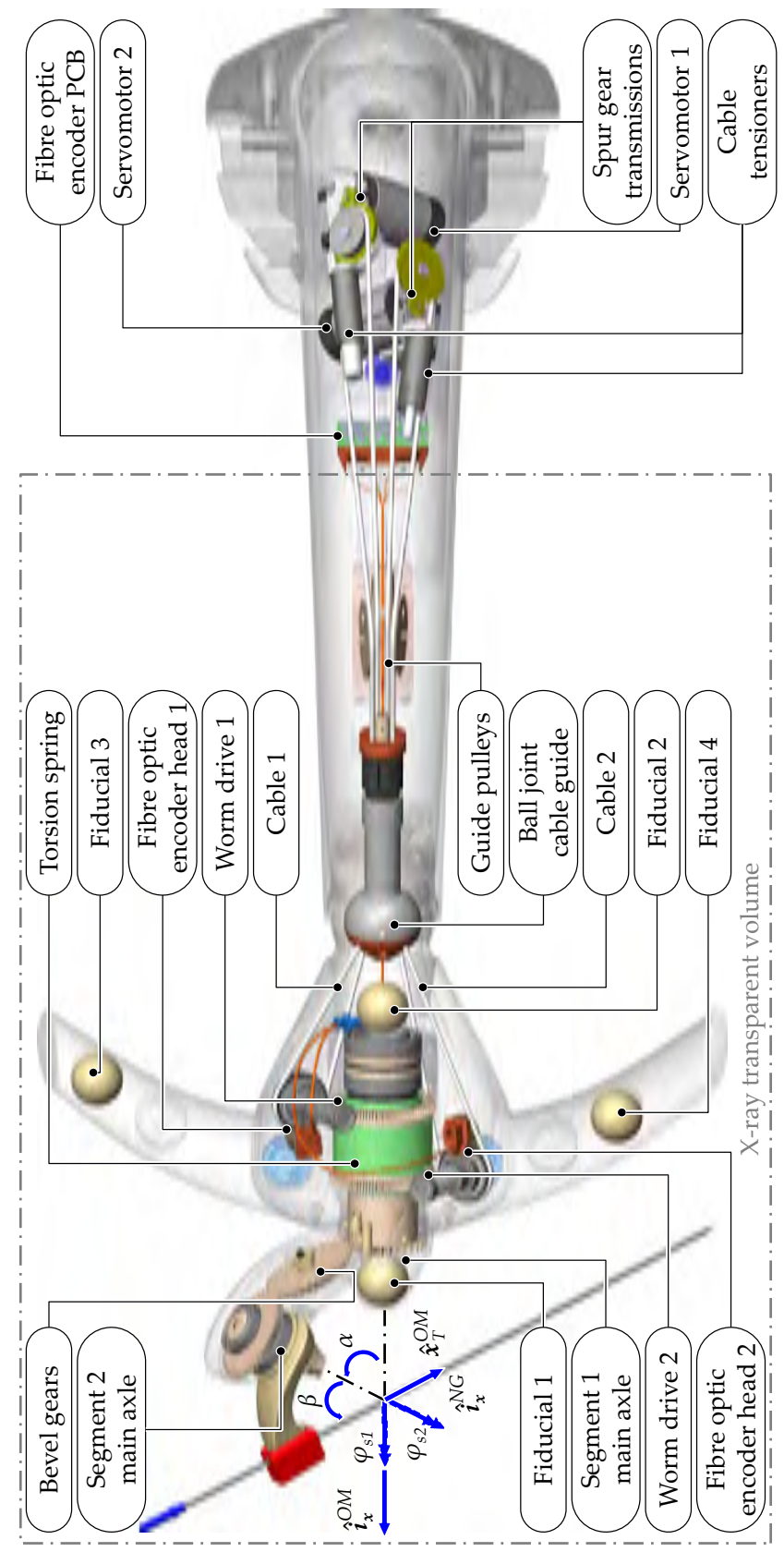

Figure 5.2: System internals, showing the fiducial markers and the drive system. 
CT coordinate systems; a fourth fiducial marker is added to decrease registration errors by positional averaging.

To locate the fiducial markers in the images, 3D object segmentation by pixel value thresholding is applied, followed by filtering of found objects based on their volume and volumetric moments with respect to their centres about three orthogonal axes. Two fiducial markers are glued to the ends of the glass main axle of segment 1 and two fiducial markers are incorporated in the handlebars of the OM main body, which is 3D printed by selective laser sintering (SLS) of a composite of nylon, glass fibre and aluminium by the name Rigid GF (Fig. 5.2). The aluminium oxide of the fiducial markers provides good image contrast with both glass and Rigid GF, facilitating segmentation.

The centre coordinates $x_{i}^{C T}$ of a fiducial marker $i$ with respect to the CT coordinate system are calculated as the centre of volume of the object identified as a fiducial marker. Given the known position $x_{i}^{O M}$ of each fiducial marker $i$ with respect to the $O M$ coordinate system, determining which point reference corresponds to which fiducial marker is based on the distances to their collective mean position. The best fit of the OM's position $x_{O M}^{C T}$ and orientation in terms of four Euler parameters $\lambda_{O M}^{C T}$ with respect to the CT coordinate system are found at a minimum of cost function $J$, which sums the squared values of the fiducial registration error (FRE) $e_{i}\left(x_{O M}^{C T}, \lambda_{O M}^{C T}\right)$ of each fiducial marker $i$ :

$$
J\left(x_{O M}^{C T}, \lambda_{O M}^{C T}\right)=\sum_{i=1}^{4}\left(e_{i}\left(x_{O M}^{C T}, \lambda_{O M}^{C T}\right)\right)^{2},
$$

with:

$$
\begin{aligned}
& e_{i}\left(x_{O M}^{C T}, \lambda_{O M}^{C T}\right)= \\
& \left\|x_{i}^{C T}-\left(x_{O M}^{C T}+R^{O M: C T}\left(\lambda_{O M}^{C T}\right) \cdot x_{i}^{O M}\right)\right\| .
\end{aligned}
$$

Here, $\boldsymbol{R}^{\mathrm{OM:CT}}\left(\lambda_{O M}^{C T}\right)$ is a rotation matrix which performs the rotation of coordinates from the $\mathrm{OM}$ to the $\mathrm{CT}$ coordinate system. The Euler parameters $\lambda_{O M}^{C T}$ must satisfy their constraint equation:

$$
\left(\lambda_{O M}^{C T}\right)^{T} \lambda_{O M}^{C T}-1=0,
$$

which is taken into account by introduction of Lagrange multiplier $\mu$ and extension of the cost function (Eq. 5.1) to:

$$
\begin{aligned}
& J^{*}\left(x_{O M}^{C T}, \lambda_{O M}^{C T}, \mu\right)= \\
& J\left(x_{O M}^{C T}, \lambda_{O M}^{C T}\right)+\mu\left(\left(\lambda_{O M}^{C T}\right)^{T} \lambda_{O M}^{C T}-1\right) .
\end{aligned}
$$

At the minimum of $J^{*}\left(x_{O M}^{C T}, \lambda_{O M}^{C T}, \mu\right)$, its partial derivatives (denoted using the nabla operator $\nabla$ ) to $x_{O M}^{C T}, \lambda_{O M}^{C T}$ and $\mu$ must be zero: 


$$
\nabla J^{*}\left(x_{O M}^{C T}, \lambda_{O M}^{C T}, \mu\right)=\mathbf{0} .
$$

Equation 5.5 is solved numerically for $x_{O M}^{C T}$ and $\lambda_{O M}^{C T}$ (and $\mu$ ) using the iterative Newton-Raphson method:

$$
\left\{\begin{array}{c}
x_{O M}^{C T} \\
\lambda_{O M}^{C T} \\
\mu
\end{array}\right\}=\left\{\begin{array}{c}
x_{O M}^{C T} \\
\lambda_{O M}^{C T} \\
\mu
\end{array}\right\} \frac{\nabla^{2} J^{*}\left(x_{O M}^{C T}, \lambda_{O M}^{C T}, \mu\right)^{j}}{\nabla J^{*}\left(x_{O M}^{C T}, \lambda_{O M}^{C T}, \mu\right)^{j}},
$$

where $j$ denotes the iteration step. The initial configuration of the iterative method is defined by (1) coincidence of $x_{1}^{\mathrm{OM}}$ with $x_{1}^{\mathrm{CT}}$, (2) coincidence of $x_{2}^{\mathrm{OM}}$ with the axis through $x_{1}^{C T}$ and $x_{2}^{C T}$, and (3) coincidence of $x_{3}^{O M}$ with the plane spanned by $x_{1}^{C T}, x_{2}^{C T}$ and $x_{3}^{C T}$. The iteration is stopped once Eq. 5.5 is satisfied by a numerical tolerance of $1 \cdot 10^{-6}$ for each partial derivative.

\section{Performance analysis}

The error contribution of the registration system was experimentally determined by CT scanning of a PVC dummy frame which holds four fiducial markers at identical relative positions as in the OM (Fig. 5.3). The dummy frame holds a fifth fiducial marker which serves as a point reference to a target position $x_{T}^{\mathrm{OM}}$ which corresponds to a needle tip in the centre of the conical action range of the aiming mechanism and at $250 \mathrm{~mm}$ from a virtual RCM at $x_{R C M}^{O M}$. The OM coordinate system is conveniently defined to coincide with the RCM, with its $x$-direction defined by the line from markers 2 to 1 , and its $y$-direction by the perpendicular line to markers 3 . The relative positions $x_{i}^{\mathrm{OM}}(i=[1,2,3,4])$ and $x_{T}^{\mathrm{OM}}$ of the fiducial markers were determined using a FARO [212] Gage Plus measuring arm with a specified volumetric length measuring uncertainty of $5 \mu \mathrm{m}$ :

$$
\begin{aligned}
& {\left[\begin{array}{lccccc}
x_{R C M}^{O M} & x_{1}^{O M} & x_{2}^{O M} & x_{3}^{O M} & x_{4}^{O M} & x_{T}^{O M}
\end{array}\right]=} \\
& {\left[\begin{array}{cccccc}
0 & -48.66 & -156.23 & -123.86 & -112.37 & 94.56 \\
0 & 0 & 0 & 111.80 & -88.13 & 39.01 \\
0 & 0 & 0 & 0 & 18.83 & 227.69
\end{array}\right] .}
\end{aligned}
$$

A Siemens [35] Somatom Force CT scanner was used with a scanning protocol for the liver. The reconstructed images are $512 \times 512$ pixels with values 0 to 4095 (12 bits), a pixel spacing of $0.84 \times 0.84 \mathrm{~mm}$ and an image spacing of $1.5 \mathrm{~mm}$. A 


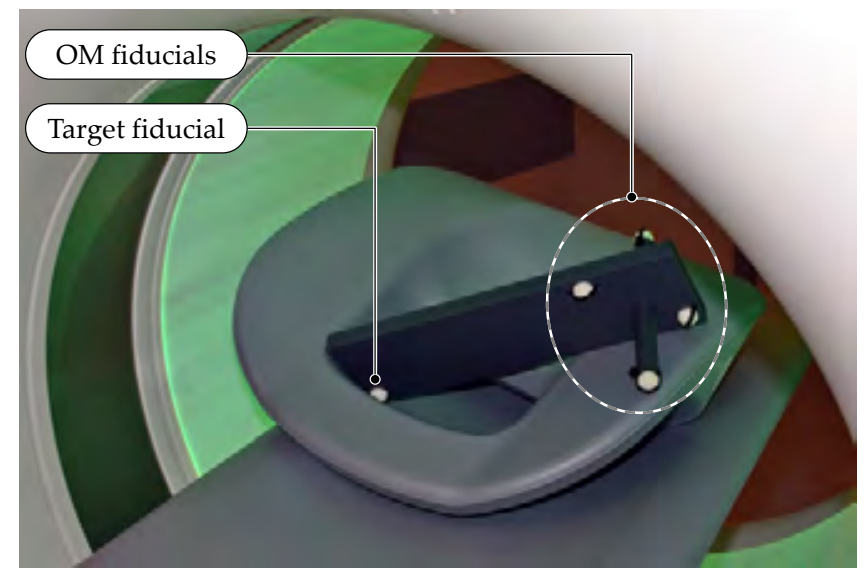

(a) CT scanning of the dummy frame on a foam support.

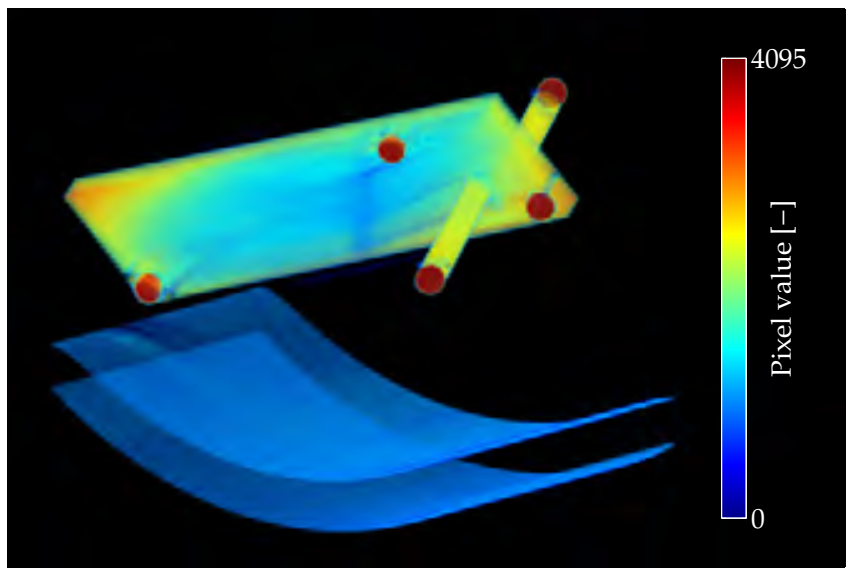

(b) 3D CT image of the dummy frame.

Figure 5.3: CT scanning of the dummy frame to determine the contribution of the registration system to the placement error.

total of $8 \mathrm{CT}$ scans were performed of the dummy frame in various positions and orientations. For each scan, image segmentation was performed using minimum pixel value threshold levels ranging from 2900 to 4050 in steps of 50. For each threshold value, found objects were filtered to match the volume and volumetric moments of an equivalent fiducial marker diameter between 15 and $22 \mathrm{~mm}$. The registration of the OM coordinate system with that of the CT was performed based on the four fiducial markers $x_{i}^{C T}(i=[1,2,3,4])$, yielding position $x_{O M}^{C T}$ and orientation $\lambda_{O M}^{C T}$. The CT coordinates of the fifth fiducial marker, $x_{T}^{C T}$, allow for calculation of the target registration error (TRE) $e_{T}$ the contribution to the place- 


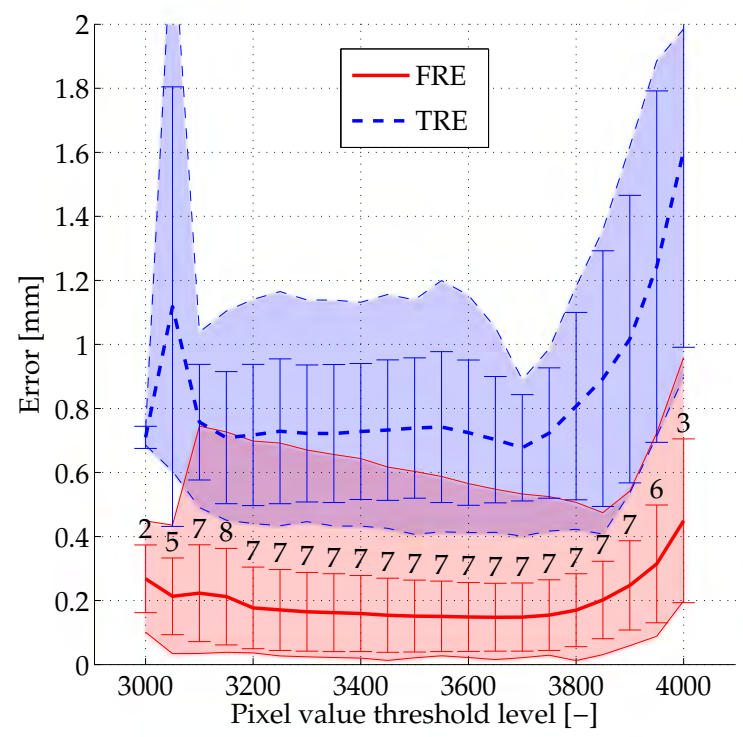

Figure 5.4: Mean value, standard deviation, minimum and maximum (filled areas) of fiducial registration errors (FRE, Eq. 5.2) and target registration error (TRE, Eq. 5.8) as a function of pixel value threshold level used for segmentation, accompanied by the number of successful segmentations out of $8 \mathrm{CT}$ scans.

ment error by the registration system:

$$
e_{T}\left(x_{O M}^{C T}, \lambda_{O M}^{C T}\right)=\left\|x_{T}^{C T}-\left(x_{O M}^{C T}+\boldsymbol{R}^{O M: C T}\left(\lambda_{O M}^{C T}\right) \cdot x_{T}^{O M}\right)\right\| .
$$

The FRE and TRE were determined for each registration.

\subsubsection{Results}

Image segmentation was successful for $\geqslant 7$ out of 8 scans in the pixel value threshold level range of 3100 to 3900 . No segmentation was successful outside the range of 3000 to 4000 . For 1 scan, segmentation was successful only in the range of 3000 to 3150 , due to image distortion by intersection of the full length of the dummy frame with the image plane. In general, segmentation fails for too low pixel value threshold levels because imaged fiducial markers and dummy frame become merged, whereas segmentation fails for too high levels because the reduction of pixels causes objects to no longer fulfil size criteria to be identified as a fiducial marker. 
The mean value, standard deviation, and minimum and maximum values of the FRE and TRE for the registrations following successful segmentation are plotted in Fig. 5.4 as a function of pixel value threshold level. The FRE and TRE of all registrations are $0.19 \pm 0.14 \mathrm{~mm}$ ( \pm standard deviation) and $0.81 \pm 0.34 \mathrm{~mm}$ with a maximum of $0.96 \mathrm{~mm}$ and $2.32 \mathrm{~mm}$ respectively.

\subsection{The drive system}

In this section, the design of drive system of the 2-DOFs mechanism to aim the needle guide is presented and its contribution to the placement error is quantified.

\subsubsection{Materials \& methods}

The aiming mechanism consists of segment 1 connected via a rotatory joint to the main body of the OM, and segment 2 connected via a rotatory joint to segment 1 (Fig. 5.1). The needle guide is incorporated in segment 2 . The two joint axes and the axial trajectory of the needle guide coincide in the same point outside the OM, creating the RCM. The arc angles between the in- and outgoing axes of segment 1 and 2 are $\alpha=60^{\circ}$ and $\beta=52.5^{\circ}$ respectively, designed to create an action range for the needle trajectory which covers a $90^{\circ}$ cone.

The rotational angles of segment $1\left(\varphi_{s 1}\right)$ and $2\left(\varphi_{s 2}\right)$ to be set by the drive system are geometrically calculated using the OM position $x_{O M}^{C T}$ and orientation $\lambda_{O M}^{C T}$ (obtained by numerically solving Eq. 5.6) and a target position $x_{T}^{C T}$, user-specified via the GUI. The target coordinates are first transformed to the OM coordinate system:

$$
\boldsymbol{x}_{T}^{\mathrm{OM}}=\left(\boldsymbol{R}^{\mathrm{OM}: C T}\left(\lambda_{\mathrm{OM}}^{\mathrm{CT}}\right)\right)^{-1}\left(\boldsymbol{x}_{T}^{C T}-\boldsymbol{x}_{\mathrm{OM}}^{\mathrm{CT}}\right) .
$$

The insertion depth from RCM to target is readily calculated as the Euclidean norm of the vector, $\left\|x_{T}^{\mathrm{OM}}\right\|$. The needle guide coordinate system $(N G)$ is defined such that its $x$-axis is the direction of the guidance, and its $y$-axis is orthogonal to the ingoing and outgoing axes of segment 2 . The normalized target vector $\hat{x}_{T}^{O M}$ is equated to the unit vector $\hat{i}_{x}^{N G}$ in the $x$-direction via four subsequent coordinate system rotations about the $z-, x-, z-$ and $x$-axis (Fig. 5.2):

$$
\hat{x}_{T}^{O M}=\boldsymbol{R}^{x}\left(\varphi_{s 1}\right) \cdot \boldsymbol{R}^{z}(\alpha) \cdot \boldsymbol{R}^{x}\left(\varphi_{s 2}\right) \cdot \boldsymbol{R}^{z}(\beta) \cdot \hat{i}_{x}^{N G},
$$

with $\boldsymbol{R}^{x}$ and $\boldsymbol{R}^{z}$ rotation matrices for rotation about the $x$ - and $z$-axis respectively. If $\hat{x}_{T}^{\mathrm{OM}}$ is within the action range of the aiming mechanism, solving Eq. 5.10 for $\varphi_{s 1}$ and $\varphi_{s 2}$ yields two solution combinations, corresponding to two segment configurations mirrored by the plane spanned by vectors $\hat{x}_{T}^{O M}$ and $\hat{i}_{x}^{O M}$, the unit vector in the $x$-direction of the OM coordinates system. 


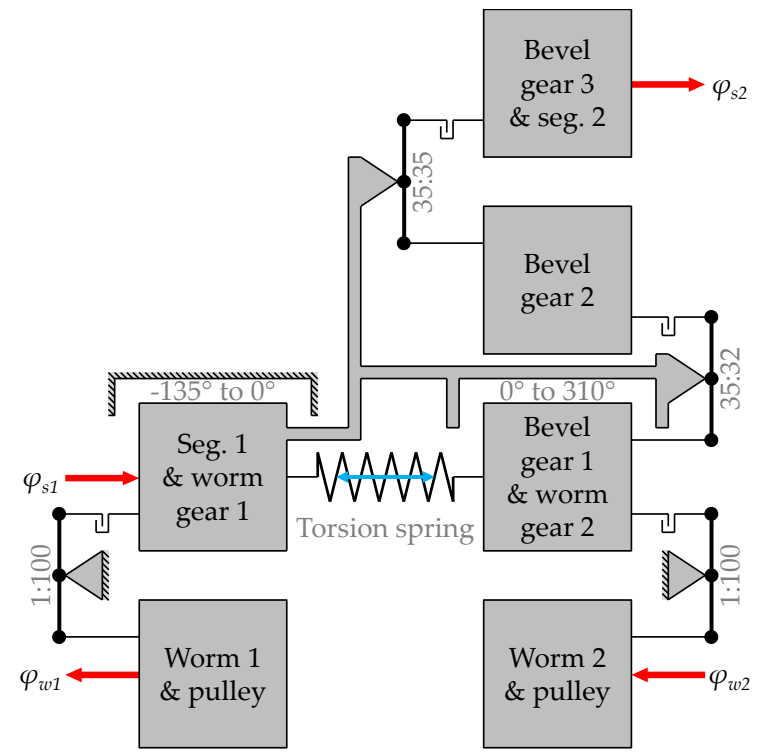

Figure 5.5: Kinematic diagram of the transmission from worms to segments, converted from the rotational to the translational domain for illustrative purposes. Diagonal cross-hatching indicates connection to the fixed frame of reference of the main body of the OM.

Figure 5.2 illustrates the drive system of the two segments. The rotation of segment 2 is coupled via three bevel gears, ratio 32:35:35, incorporated in segment 1 to the rotation of a hollow axle which is concentric with the axle of segment 1 . Each of these two concentric axles is rotated by a non-backdriveable 1:100 worm drive, such that the needle guide maintains its angles in case of a power failure. Backlash in the two worm drives is eliminated by a preload applied by a single torsion spring mounted in-between the two concentric axles.

The drive system is actuated by two electrical servomotors (Maxon [182] REmax 24, $11 \mathrm{~W}$ ) placed in the lower part of the LM arm, to maintain X-ray transparency of the OM and upper part of the LM arm. Each motor drives one of the two worms, via a 7:24 spur gear transmission, pulleys and an endless Dyneema cable guided through the 3-DOFs ball joint 2 . The surfaces of the ingoing and outgoing pulleys are rubberised to achieve sufficient traction by a high coefficient of friction with the Dyneema cable. This allows for a reduced cable tension to transmit the required torque, which in turn reduces cable wear as well as bearing loads and friction, and consequently improves drive system efficiency. The motors are equipped with incremental rotatory encoders of $4 \times 500$ counts per revolution for motion control using Elmo [213] SimplIQ motor controllers.

To account for slip and variations in the cable transmission, two rotatory en- 
coders with $4 \times 96$ counts per revolution are added to measure the angles of the worms $\left(\varphi_{w 1}\right.$ and $\left.\varphi_{w 2}\right)$ and control the angles of the segments $\left(\varphi_{s 1}\right.$ and $\left.\varphi_{s 2}\right)$ via Eq. 5.11. During motion control to aim the needle guide, the setpoints of the servomotors are updated stepwise until the worms have reached their target values as measured by the encoders on the worms. In consideration of X-ray transparency, custom encoders were developed which use plastic optical fibres to transmit light from light-emitting diodes (LEDs) to photodiodes on a printed circuit board (PCB) outside the X-ray transparent volume in the lower part of the LM arm, via reflective incremental code wheels on the worm axles.

A kinematic diagram of the transmission from worms to segments is shown in Fig. 5.5. The corresponding geometric transfer function between the rotation of the two worms $\left(\varphi_{w 1}\right.$ and $\left.\varphi_{w 2}\right)$ and segments $\left(\varphi_{s 1}\right.$ and $\left.\varphi_{s 2}\right)$ is as follows:

$$
\left\{\begin{array}{l}
\varphi_{s 1} \\
\varphi_{s 2}
\end{array}\right\}=1 / 100\left[\begin{array}{cc}
1 & 0 \\
-32 / 35 & 32 / 35
\end{array}\right]\left\{\begin{array}{l}
\varphi_{w 1} \\
\varphi_{w 2}
\end{array}\right\} .
$$

Home angles for the main axles of segments 1 and 2 are defined by mechanical stops on each of the two concentric axles and are detected through motor current thresholding. The geometry of the segments and their angles in home position with respect to the coordinate system of the OM are determined using a FARO [212] Gage Plus measuring arm.

Material selection for the components of the drive system inside the intended $\mathrm{CT}$ scanned volume is restricted by the requirement on X-ray transparency. The two concentric axles driving segment 1 and 2 are borosilicate glass and aluminium oxide respectively. The main axles of segment 1 and 2 and both worms are mounted in silicon nitride ball bearings. Each worm, its coaxial pulley and inner ball bearing rings are merged into single silicon nitride parts for compactness and precision. The three bevel gears and worm gears are made of PEEK, which is more X-ray transparent than ceramics and more easily processed by milling. The torsion spring in-between the two concentric axles is custom moulded from glass fibre reinforced epoxy, which is X-ray transparent and does not creep under the applied preload.

\section{Performance analysis}

The error contribution of the drive system is affected by encoder resolutions, and transmission errors due to geometrical deviations from the nominal dimensions of the worm drives and bevel gears, and their deformation under preload and load transmission. To quantify the transmission errors, the input angles of the worms measured by the integrated fibre-optic encoders were compared to the output angles of the segments.

Two experiment setups were hereto used, in which an INDUcoder [214] ED58 incremental rotatory encoder with $4 \times 32000$ counts per revolution was intro- 


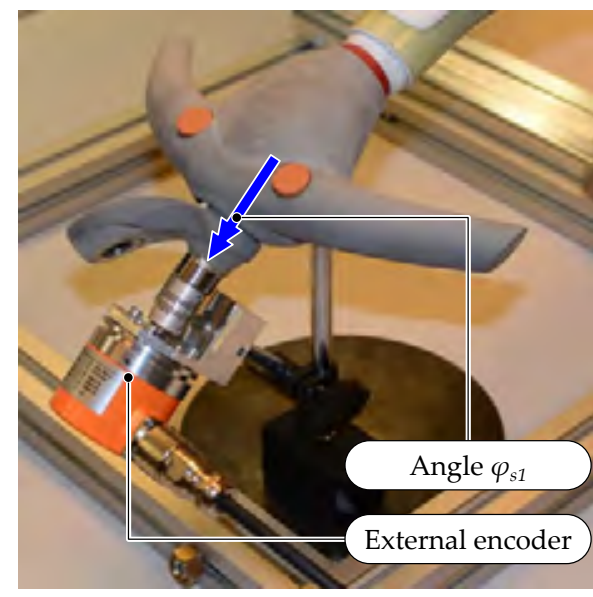

(a) Measuring the angle of segment 1.

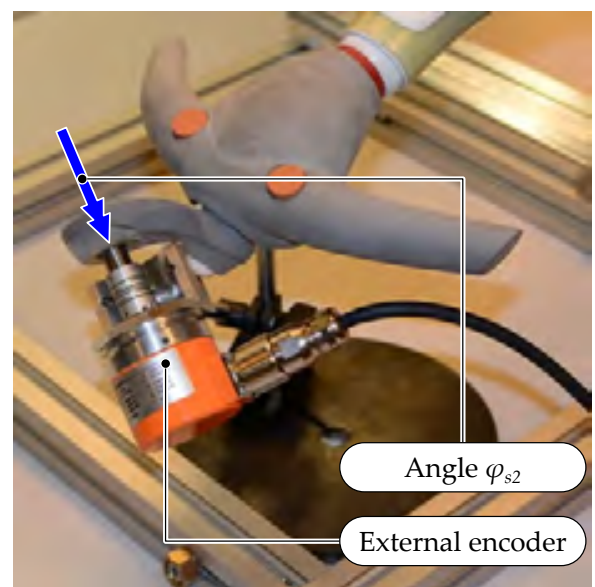

(b) Measuring the angle of segment 2.

Figure 5.6: Experiment setups to determine the error contribution of the drive system.

duced to externally measure the angle of the axle of segment 1 and 2 respectively (Fig. 5.6). In both setups, the LM was in locked state to fix the position and orientation of the $\mathrm{OM}$ to a table mounted aluminium frame. The external encoder was supported by a mechanical arm and connected to the respective segment axle via a flexible slit coupling. Two experiments were performed:

1. Using the first setup (Fig. 5.6a), segment 1 was homed, with segment 2 angled such that its home and end stops did not interfere with the motion of segment 1 , and all encoder values were set to zero. The angle of segment 1 was then incremented by servomotor 1 via the Dyneema cable in 250 steps over an angular range of $-135^{\circ}$ and back 5 times, during which the output of the worm 1 encoder and the external encoder on the axle of segment 1 were acquired.

2. Using the second setup (Fig. 5.6b), both segments 1 and 2 were homed, and all encoder values were set to zero. The angle of segment 2 was then incremented by servomotor 2 via the Dyneema cable in 250 steps over an angular range of $303^{\circ}$ and back 5 times, during which the output of the worm 2 encoder and the external encoder on the axle of segment 2 were acquired.

Motion of the drive system was paused after each increment in both experiments to ensure synchronous measurement sample acquisition of the encoders. Each experiment was repeated 3 times. The angular transmission errors of the drive 


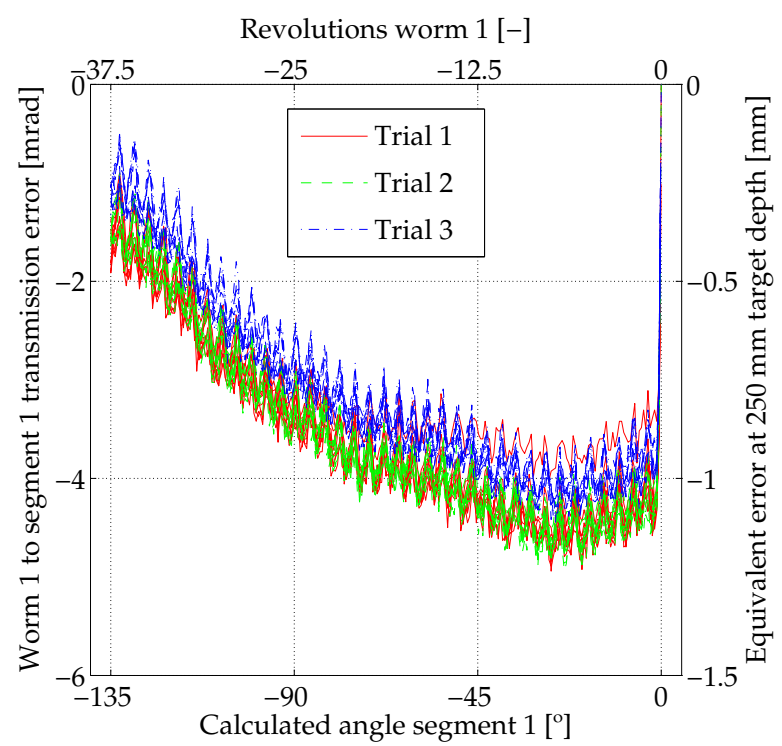

(a) Transmission errors for worm 1 to segment 1 .

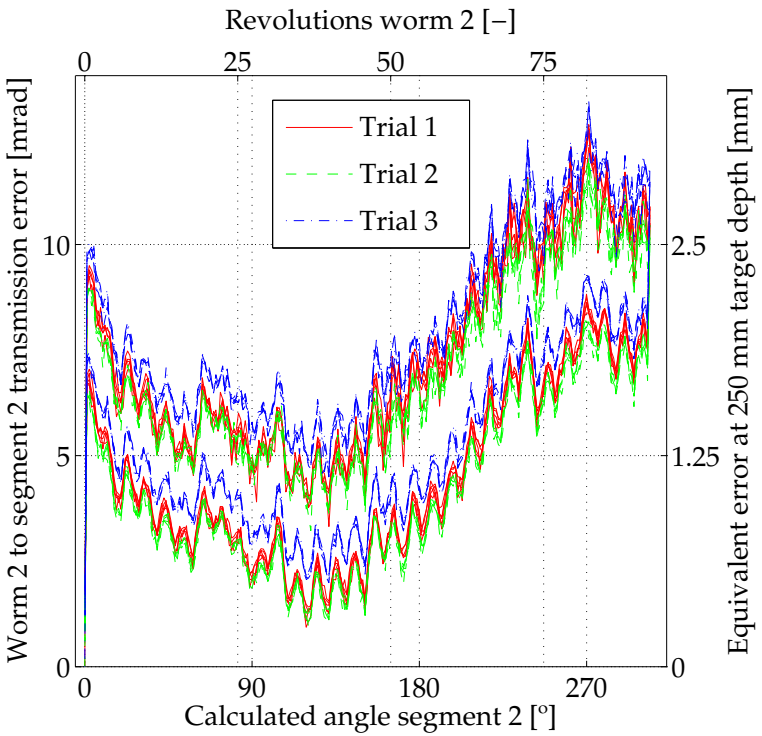

(b) Transmission errors for worm 2 to segment 2.

Figure 5.7: Angular errors of the drive system and equivalent placement errors at a target depth of $250 \mathrm{~mm}$. 
system are defined as the externally measured angles of segment 1 and 2 minus the nominal segment angles calculated by Eq. 5.11 using the measured worm angles. The equivalent placement errors at a target depth of $250 \mathrm{~mm}$ were derived from these angular errors.

\subsubsection{Results}

Figure 5.7 plots the results of the two experiments. Iteration of the measurements show the transmission errors are repeatable as a function of segment angles. An instant error offset is found for each set of measurement samples at the home angles $\left(0^{\circ}\right)$ of both segments. This is attributed to compliance of the drive system between worm and segment home stops. Minor variation in these offsets per segment is attributed to variation in torque transmitted by the cable drive to the worm for homing. The offsets are larger for segment 2 due to the larger compliance of its home stop design.

Eccentricities can be observed in the transmissions to both segments. The high frequent eccentricities are attributed to the worms, based on their periodicity being equal to the revolutions of the worm. The lower frequent eccentricities are attributed to the worm gears in the transmissions to both segment 1 and 2, and to the bevel gears in the transmission to segment 2 .

An offset of $2.5 \mathrm{mrad}$ between the going and returning angular error to segment 2 is attributed to backlash in the bevel gear transmission.

The measurements yield a maximum contribution to the placement error of $1.2 \mathrm{~mm}$ and $3.3 \mathrm{~mm}$ at $250 \mathrm{~mm}$ target depth for segment 1 and segment 2 respectively. The repeatability of the transmission errors allows for partial compensation by adjustment of the home angles of the segments.

\subsection{The structural link}

The function of the structural link between the needle guide and CT table is to hold the needle guide in place under external loads in locked state of the LM. A force up to $10 \mathrm{~N}$ is estimated to be applied by the physician when using the $\mathrm{OM}$ main body for hand support during manual insertion. A lateral force up to $4 \mathrm{~N}$ is estimated to be applied via the needle on the needle guide during insertion. These estimates are based on load case simulations on a weighing scale. The compliances of the structural link relate the loads to deviation of the needle guide trajectory due to deformation, and consequently to its contribution to the placement error. In this section, the design of the structural link is presented and its compliances and contribution to the placement error under given loads are quantified. 


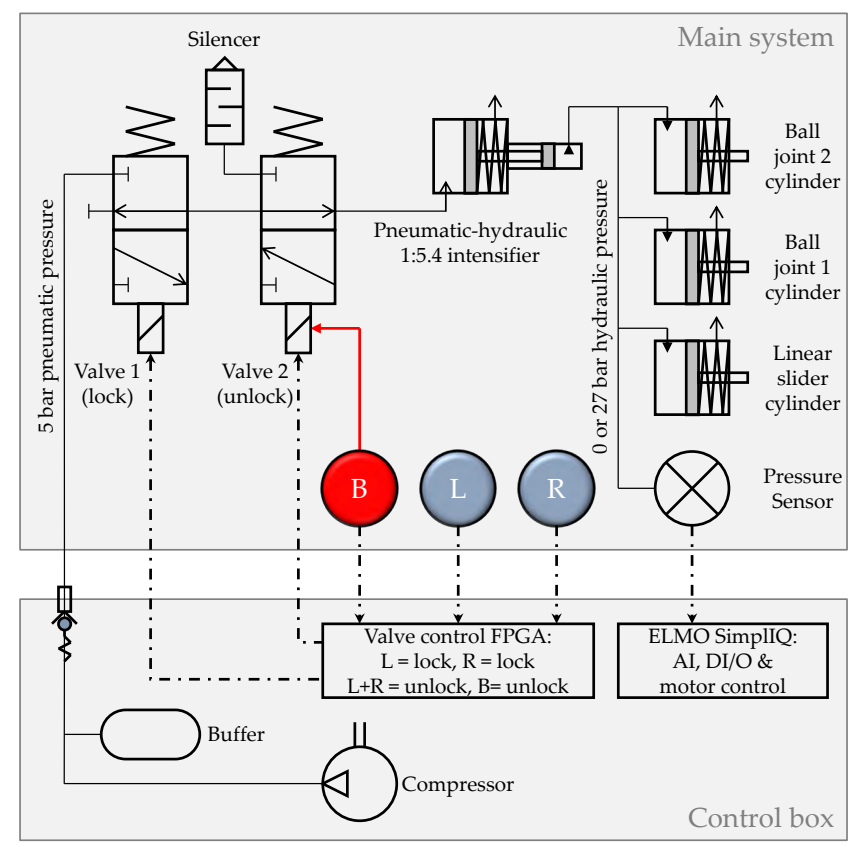

Figure 5.8: Diagram of the pneumatic-hydraulic locking system, controlled by electrical lock/unlock buttons ' $L$ ' and ' $R$ ' and mechanical backup unlock button 'B'.

\subsubsection{Materials \& methods}

The structural link includes the linear slider, ball joint 1 and ball joint 2 (Fig. 5.1), which can simultaneously be locked using a pneumatic-hydraulic system (Fig. 5.8, Fig. 5.9). A compressor in an external control box supplies 5 bar pneumatic pressure to the LM foot, passed via two valves to a 1:5.4 intensifier which converts it to 27 bar hydraulic pressure. This pressure acts on three pistons, each of which friction lock one of the three joints. The lock and unlock valves of the locking system are electrically controlled using two push buttons on the OM, via a field-programmable gate array (FPGA) in the control box. A back-up button in the LM foot provides both direct mechanical and electrical control of the unlock valve. The system's software monitors the (un)locked state of the OM by measuring the hydraulic pressure using a sensor connected to the analog input (AI) of the Elmo [213] SimplIQ motor controllers, which are also used for motor control and digital in- and outputs (DI/O). The moment on ball joint 1 due to gravitational forces on the LM arm and the OM is compensated by an eccentric spring mechanism to prevent them from falling onto the patient or CT table when unlocked. 


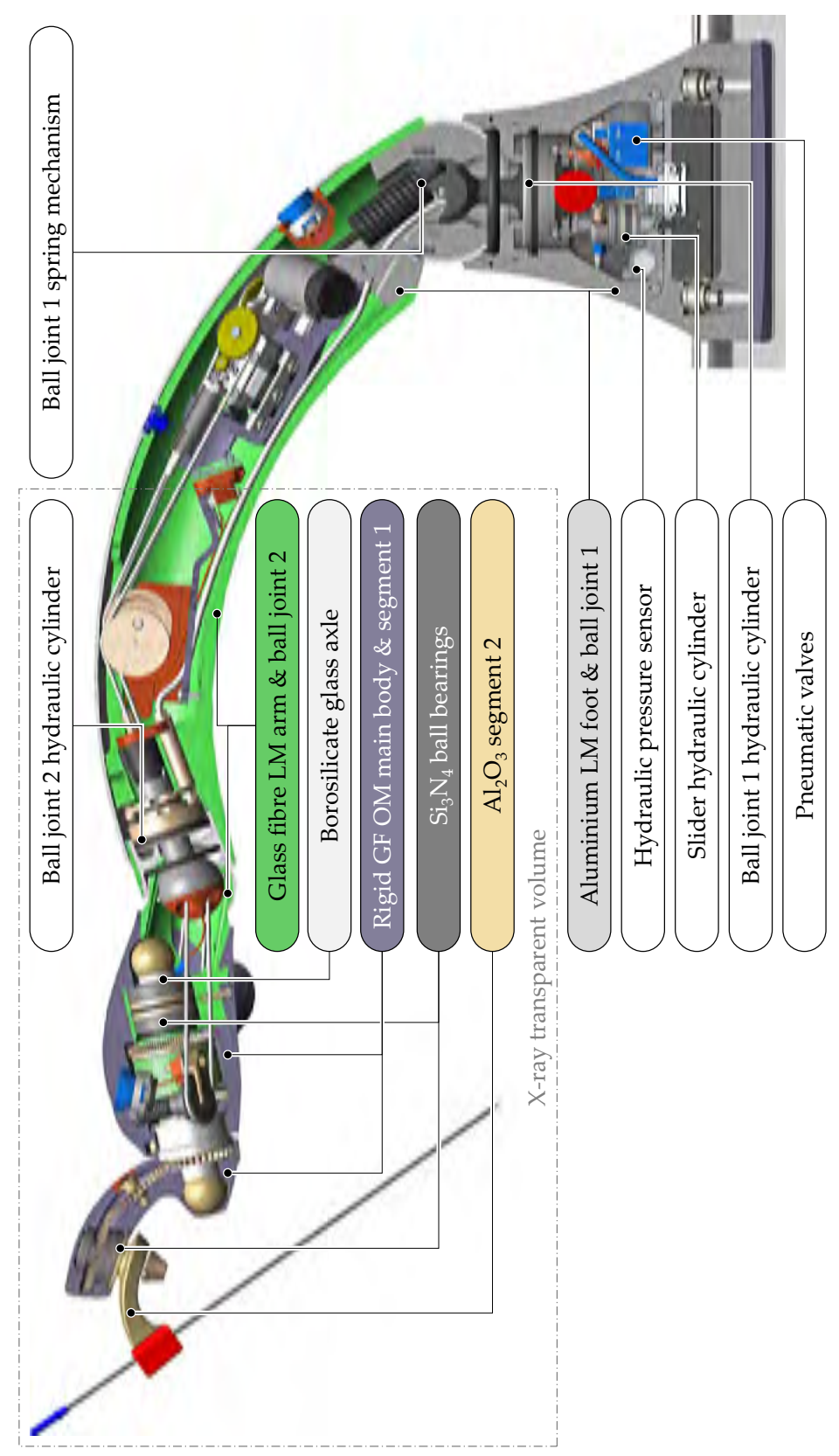

Figure 5.9: Cross section of the system with labelled components of the locking system and materials forming the structural link. 
The compliance of the structural link is constituted by its geometry and the mechanical properties of its materials. The geometry was designed based on constraints on the available space between patient and CT scanner bore and requirements on the range and freedom of movement of the OM. In addition to mechanical properties, considerations for material selection are radiolucency, sterilizability, manufacturability and production costs. Figure 5.9 indicates the materials of the main components that form the structural link, which include aluminium, aluminium oxide, glass fibre reinforced epoxy and SLS 3D printed Rigid GF.

\section{Performance analysis}

The compliances were determined by measuring the deflection of the needle guide under the gravitational force of an external mass. The pose of the structural link changes its geometry and consequently its compliance. Two worst case poses were therefore considered, each with a specific corresponding load case.

Firstly, the OM was locked in horizontal position (Fig. 5.10a). A vertical force $F_{y}^{M B}$ was applied to the main body (MB) of the OM by external mass $m_{1}=0.999 \mathrm{~kg}$ via a string. Subscript $y$ in $F_{y}^{M B}$ indicates the directional component of the force, and superscript $M B$ indicates the position where it is applied. A tool holding two spheres was mounted in the needle guide (NG) to determine its deflection. The spheres are a distance $l=80 \mathrm{~mm}$ apart, coincide with the axial trajectory of the needle guide and one sphere coincides with the RCM. Deflections $d x_{1}$, $d x_{2}$ and $d y_{1}$ of the spheres due to load application were measured using a Käfer [215] dial gauge with $0.01 \mathrm{~mm}$ resolution. The compliances $C_{y, y}^{M B: N G}$ and $C_{y, \varphi}^{M B}: N G$ relating force $F_{y}^{M B}$ to the vertical deflection $d y^{N G}$ and angular deflection $d \varphi^{N G}$ of the needle guide respectively are defined and calculated as follows:

$$
\begin{aligned}
& C_{y, x}^{M B: N G}=\frac{\partial x^{N G}}{\partial F_{y}^{M B}}=\frac{d x_{1}}{m_{1} g}, \\
& C_{y, y}^{M B: N G}=\frac{\partial y^{N G}}{\partial F_{y}^{M B}}=\frac{d y_{1}}{m_{1} g}, \\
& C_{y, \varphi}^{M B: N G}=\frac{\partial \varphi^{N G}}{\partial F_{y}^{M B}}=\frac{\left(d x_{2}-d x_{1}\right) / l}{m_{1} g},
\end{aligned}
$$

with $g$ the gravitational acceleration. Subscripts $(y, y)$ and $(y, \varphi)$ here indicate (directional component of the applied force, directional component of the deflection). Superscript ( $M B: N G)$ indicates (position of the applied load to position of the deflection). The placement error $e^{M B}$ at target depth $L$ due to force $F_{y}^{M B}$ is calculated as the sum of orthogonal contributions due to vertical, horizontal and angular deflections of the needle guide (Fig. 5.10a): 


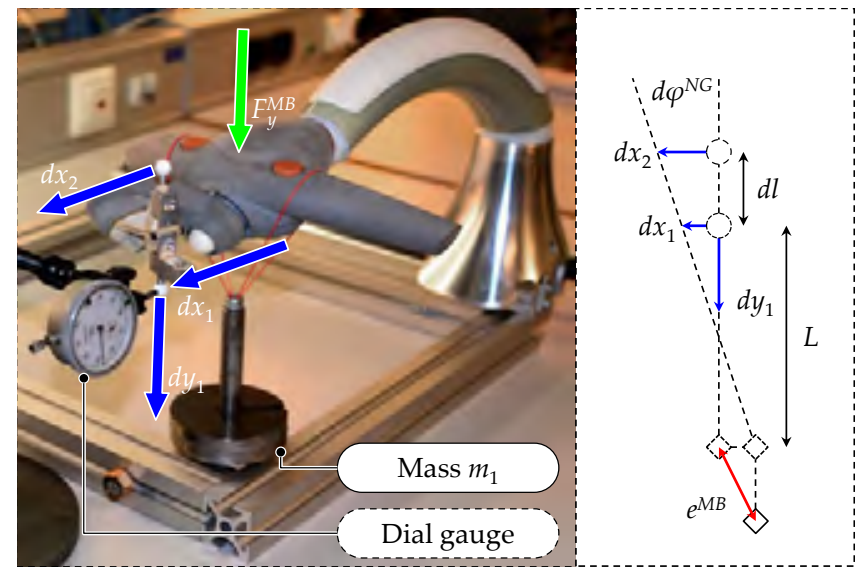

(a) Measurement of deflections $d x_{1}, d x_{2}$ and $d y_{1}$ under load $F_{y}^{M B}$ by mass $m_{1}$ on the main body $(M B)$ of the OM, and corresponding deflection diagram.

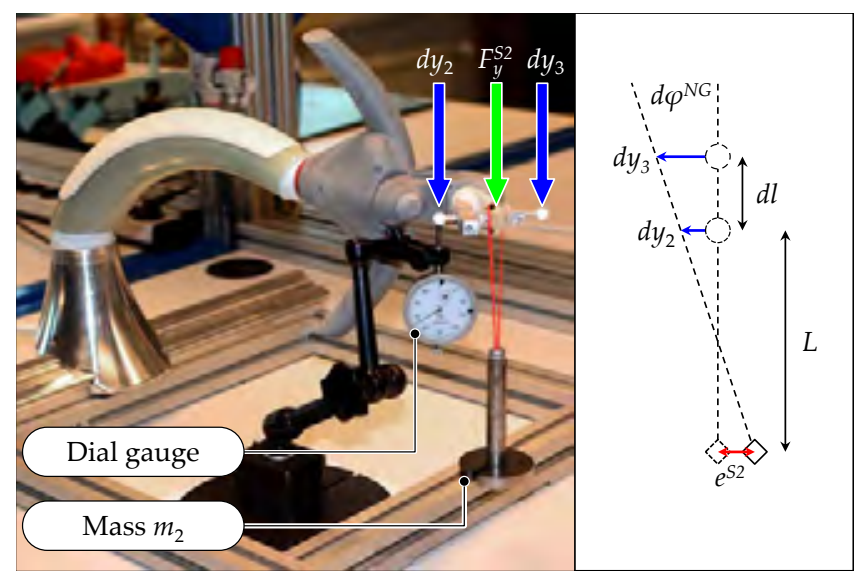

(b) Measurement of deflections $d y_{2}$ and $d y_{3}$ under load $F_{y}^{S 2}$ by mass $m_{2}$ on segment 2 (S2), and corresponding deflection diagram.

Figure 5.10: Experiment setups to determine the compliances of the structural link from needle guide to table.

$$
\begin{aligned}
e^{M B}= & \left(\left(d \varphi^{N G} L-d x_{1}\right)^{2}+\left(d y^{N G}\right)^{2}\right)^{0.5} \\
= & \left(\left(\left(C_{y, \varphi}^{M B: N G} L-C_{y, x}^{M B: N G}\right) F_{y}^{M B}\right)^{2}\right. \\
& \left.+\left(C_{y, y}^{M B: N G} F_{y}^{M B}\right)^{2}\right)^{0.5} .
\end{aligned}
$$


Secondly, the OM was locked in vertical position (Fig. 5.10b). A vertical force $F_{y}^{S 2}$ was applied at the needle guide on segment 2 (S2) by external mass $m_{2}=0.399$ $\mathrm{kg}$ via a string. Vertical deflections $d y_{2}$ and $d y_{3}$ of the spheres were subsequently measured using the dial gauge. The horizontal deflections were estimated to be negligible and ignored. The compliances $C_{y, y}^{S 2: N G}$ and $C_{y, \varphi}^{S 2}$ relating force $F_{y}^{S 2}$ to the vertical deflection $d y^{N G}$ and angular deflection $d \varphi^{N G}$ of the needle guide respectively are defined and calculated as follows:

$$
\begin{aligned}
C_{y, y}^{S 2: N G} & =\frac{\partial y^{N G}}{\partial F_{y}^{S 2}}=\frac{d y_{2}}{m_{2} g}, \\
C_{y, \varphi}^{S 2: N G} & =\frac{\partial \varphi^{N G}}{\partial F_{y}^{S 2}}=\frac{\left(d y_{3}-d y_{2}\right) / l}{m_{2} g} .
\end{aligned}
$$

The placement error $e^{S 2}$ at target depth $L$ due to force $F_{y}^{S 2}$ is calculated as the sum of the (opposite) contributions of vertical and angular deflections of the needle guide (Fig. 5.10b):

$$
\begin{aligned}
e^{S 2} & =\left\|d \varphi^{N G} L-d y^{N G}\right\| \\
& =\left\|\left(C_{y, \varphi}^{S 2: N G} L-C_{y, y}^{S 2: N G}\right) F_{y}^{S 2}\right\| .
\end{aligned}
$$

\subsubsection{Results}

The measured deflections in the two experiment setups and compliances calculated using Eq. 5.12 and 5.14 are:

$$
\begin{array}{ll}
d x_{1}=0.02 \mathrm{~mm} & C_{y, x}^{M B: N G}=2 \cdot 10^{-3} \mathrm{~mm} / \mathrm{N} \\
d x_{2}=0.07 \mathrm{~mm} & C_{y, y}^{M B: N G}=31 \cdot 10^{-3} \mathrm{~mm} / \mathrm{N} \\
d y_{1}=0.30 \mathrm{~mm} & C_{y, \varphi}^{M B: N G}=0.064 \cdot 10^{-3} \mathrm{rad} / \mathrm{N} \\
\hline & \\
d y_{2}=0.14 \mathrm{~mm} & C_{y, y}^{S 2: N G}=36 \cdot 10^{-3} \mathrm{~mm} / \mathrm{N} \\
d y_{3}=0.32 \mathrm{~mm} & C_{y, \varphi}^{S 2: N G}=0.57 \cdot 10^{-3} \mathrm{rad} / \mathrm{N}
\end{array}
$$

For estimated forces $F_{y}^{M B}=10 \mathrm{~N}$ and $F_{y}^{S 2}=4 \mathrm{~N}$ and a target depth of $L=250$ $\mathrm{mm}$, contributions to the placement error of $e^{M B}=0.34 \mathrm{~mm}$ and $e^{S 2}=0.43$ $\mathrm{mm}$ are calculated respectively using Eq. 5.13 and 5.15 for the two poses with corresponding load cases. 


\subsection{The overall system}

In this section, the placement error of the overall system integrated with a CT scanner is quantified by needle placement at targets in air. The home angles of segment 1 and 2 were adjusted in this experiment by fixed values of 2.7 and $-7.3 \mathrm{mrad}$ respectively to compensate for the transmission errors determined in section 5.3 .

\subsubsection{Materials \& methods}

The system was mounted via an aluminium frame onto the table of a Siemens [35] Somatom Force CT scanner (Fig. 5.11a). The OM was locked in a pose where targets within the $90^{\circ}$ conical action range of the needle guide on the 2-DOFs aiming mechanism and up to $250 \mathrm{~mm}$ depth from the RCM could be reached without interference with the CT gantry or table. The target for the needle tip was the centre of an external $\varnothing 20 \mathrm{~mm}$ spherical aluminium oxide fiducial marker at the end of a PVC rod. The rod was mounted in an aluminium holder, which could freely be placed and fixed to the CT table via the aluminium frame.

The volume containing the OM and the target was CT scanned using a scanning protocol for the liver, a pixel spacing of $0.98 \times 0.98 \mathrm{~mm}$ and an image spacing of $1.5 \mathrm{~mm}$ (Fig. 5.11b). The centre coordinates of the fiducial markers incorporated in the $\mathrm{OM}$ and the target fiducial marker were determined by processing of the CT images, as explained in section 5.2. Registration was performed and the target coordinates were precisely defined by the centre point of the target fiducial marker. The angles of the needle guide to align with the target and the insertion depth were calculated. The needle guide was automatically aimed accordingly. The PVC rod holding target fiducial marker was replaced by an aluminium rod with a conical end, providing a point reference to the former centre position of the target fiducial marker. A $300 \mathrm{~mm}$ long $\varnothing 2 \mathrm{~mm}$ solid steel needle with centre tip was placed in the needle guide at the calculated depth (Fig. 5.12c) and fixed by the needle clip. The placement error was measured using a calliper as the Euclidean distance between needle tip and cone apex.

Ten target positions were approached, at depths of 100 and $250 \mathrm{~mm}$, and at the centre and four quadrants of an $80^{\circ}$ cone (Fig. 5.12d). To prepare a target at a desired position, the needle guide was first set to the appropriate angles and the needle was placed and fixed in the needle guide at the appropriate depth. The aluminium rod with conical end was mounted in the holder, which was placed and fixed such that the cone apex coincides with the needle tip. The needle was then removed and the aluminium rod was substituted by the PVC rod holding the fiducial marker as a target. 


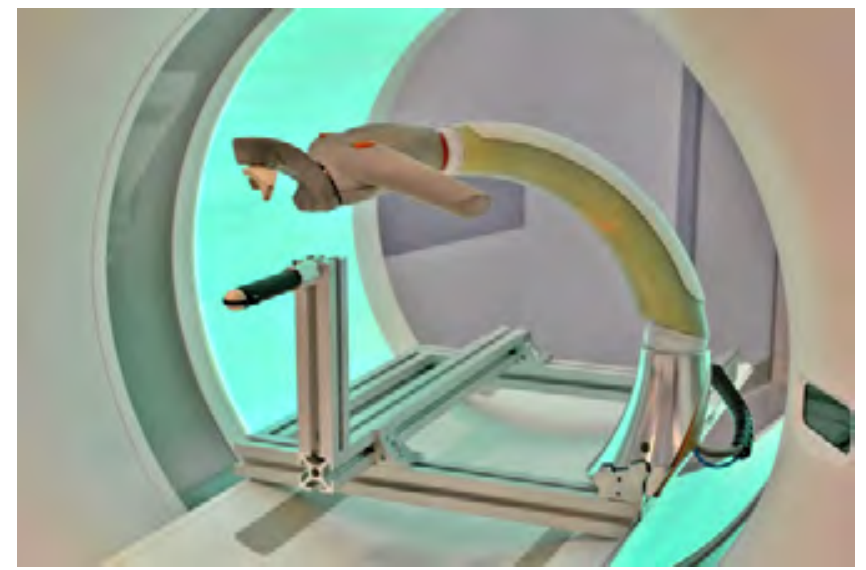

(a) The system is installed on the CT table and the OM is locked. The centre of a fiducial marker on a PVC rod serves as a target.

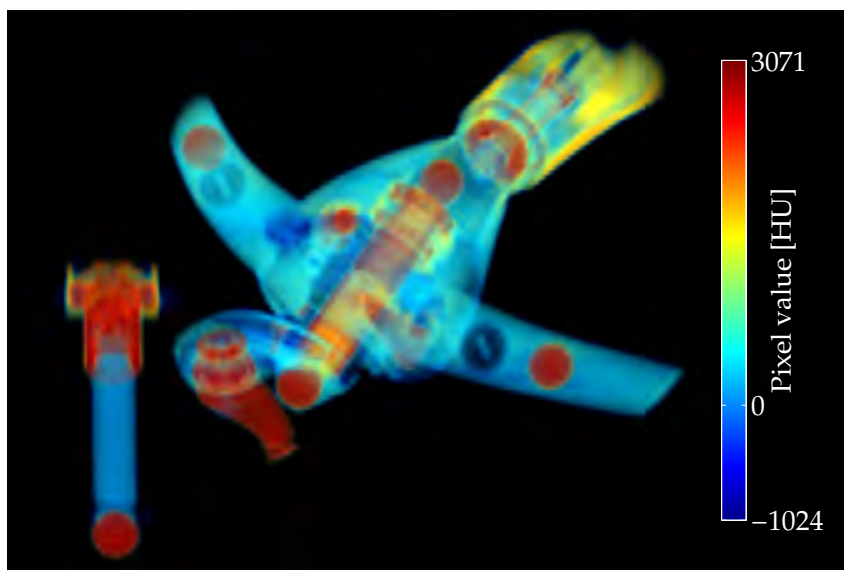

(b) 3D CT image of the OM and target fiducial marker for OM-to-CT registration and definition of the target coordinates.

Figure 5.11: Determining the placement error of the overall system.

\subsubsection{Results}

Figure 5.12d indicates the measured placement errors for the 10 target positions. A mean placement error of $1.0 \pm 0.25 \mathrm{~mm}$ with a maximum of $1.4 \mathrm{~mm}$ is found at $100 \mathrm{~mm}$ target depth, and a mean of $2.6 \pm 0.7 \mathrm{~mm}$ with a maximum of $3.5 \mathrm{~mm}$ at $250 \mathrm{~mm}$ depth. 


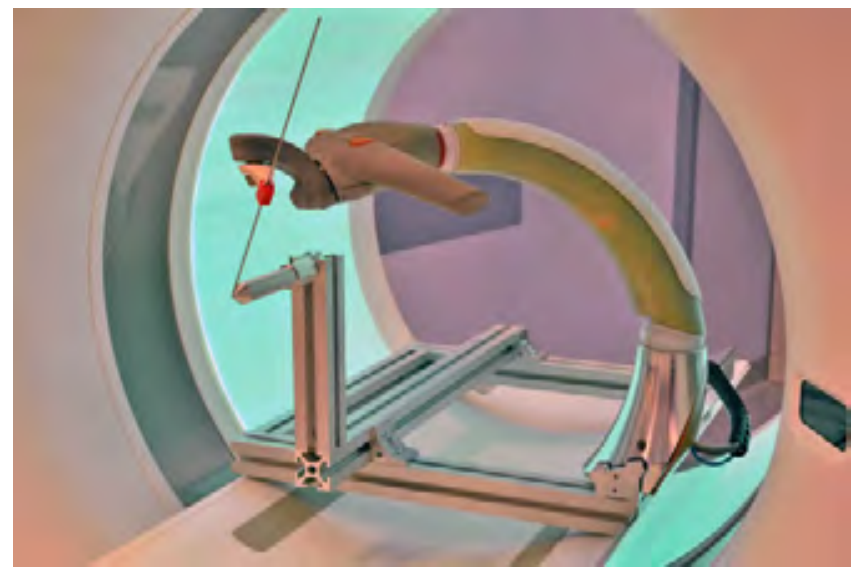

(c) The target fiducial marker is replaced by a rod with a conical end, to measure the placement error after needle placement.

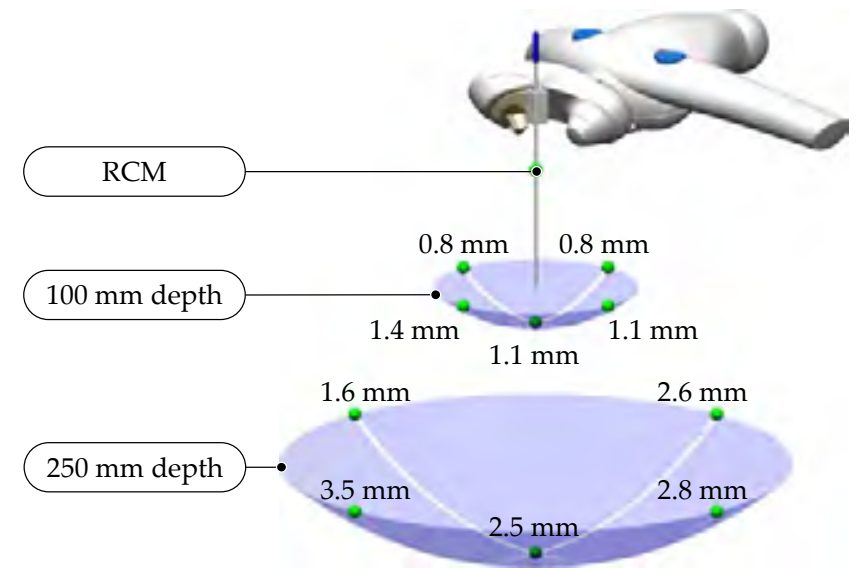

(d) Illustration of 10 target positions at 100 and $250 \mathrm{~mm}$ depth from the RCM and corresponding placement errors.

Figure 5.11: Determining the placement error of the overall system - continued.

\subsection{Discussion}

The technical design of a novel system for CT-guided needle placement has been presented and its performance in terms of placement error has been analysed. Table 5.1 provides an overview of the contribution of the three subsystems and the overall system for convenience.

The presented registration system relies on the acquisition of a set of parallel images covering the fiducial markers incorporated in the OM. Image segmentation by pixel value thresholding is employed to find the markers by image seg- 
Table 5.1: Overview of placement error contributions at $250 \mathrm{~mm}$ target depth.

\begin{tabular}{lc}
\hline (Sub)system & $\begin{array}{c}\text { Placement error } \\
\mathrm{mm}\end{array}$ \\
\hline Registration system (TRE) & $0.81 \pm 0.34$ \\
Drive system (segment 1) & $<1.2$ \\
Drive system (segment 2) & $<3.3$ \\
Structural link (OM main body loaded, 10 N) & 0.34 \\
Structural link (segment 2 loaded, 4 N) & 0.43 \\
Overall system (unloaded) & $2.6 \pm 0.7^{a}$ \\
\hline
\end{tabular}

${ }^{a}$ Home angles of segments 1 and 2 adjusted by 2.7 and $-7.3 \mathrm{mrad}$ to compensate for transmission errors.

mentation, followed by a numerical method to determine the position and orientation of the OM corresponding to a minimum of the sum of the squared fiducial registration errors. The registration system functions for any position and orientation of the OM within the field of view of CT scanner and no other calibration between system and CT scanner is required. The performance of the registration system has been analysed using a dummy frame holding four fiducial markers for registration and a fifth marker serving as a target. The successfulness and contribution to the placement error of the registration system depend on the pixel value threshold level. For successful segmentation of the Ø20 mm aluminium oxide markers and a low FRE and TRE, a pixel value threshold level between 3200 and 3700 is appropriate.

Automation of the 2-DOFs mechanism to aim the needle guide has been realized using remote actuation via endless cables to preserve sufficient $\mathrm{X}$-ray transparency. Fibre optic encoders on the worms are used for angular feedback. The transmission of the drive system from worms to segments has the largest contribution to the placement error amongst the three subsystems, attributed to home stop compliance, gear eccentricity and backlash. Home stop compliances in combination with variable cable drive torques yield variable home angle offsets. Gear eccentricity and average home angle offsets can partially be compensated for using the measured transmission errors. The performance can be improved by redesign of the home stops using X-ray transparent alternatives which do not rely on motor torque thresholding, such as carbon electrical or fibre optical limit switches.

The structural link between needle guide and CT table is composed of aluminium, glass fibre reinforced composites and ceramics to reduce its compliance. The placement error due to deformation of the structural link was determined for 
two poses and realistic load cases of the $\mathrm{OM}$ and the needle guide respectively. The physician should be instructed to avoid applying external loads in order to minimize deflection of the needle guide and the corresponding contribution to the placement error.

The performance of the overall system in terms of placement error in air does not exceed the $\leqslant 5 \mathrm{~mm}$ error specified as a clinical user requirement for needle placement in tissue, up to an extreme target depth of $250 \mathrm{~mm}$. Additional contributors to the needle tip placement error beyond control of the system are needle deflection and tissue displacement. Given the precise alignment of the needle guide with a target, needles with a symmetrical tip are recommended to follow a straight trajectory. To minimize deviation of the target tissue from the specified static target coordinates due to respiration, CT imaging and needle insertion should be performed during a breath-hold in the same respiratory phase, conform current clinical practise. Phantom and patient studies are required to validate the full performance of the system. 


\title{
CHAPTER 6
}

\section{Validation of a system for CT-guided needle placement in the thorax and abdomen - a phantom study}

\author{
Maarten M. Arnolli, Wout J. Heerink, Jan Pieter Pennings, \\ Dannis M. Brouwer, Ivo A.M.J. Broeders and Koert P. de Jong \\ In preparation for submission to Radiology, Radiological Society of North America.
}

\begin{abstract}
Background A system for CT-guided needle placement was developed which provides precise alignment of a needle guide to reach a target in a single manual insertion. The objective of this work is to validate the system by a performance comparison of its guided method and the conventional freehand method.
\end{abstract}

Methods Two experienced physicians employed both methods to reach targets at $120 \mathrm{~mm}$ depth via in-plane and $30^{\circ}$ out-of-plane paths in a gelatin phantom, with a goal to reach $\leqslant 5 \mathrm{~mm}$ Euclidean error in $\leqslant 5$ needle manipulations.

Results Guided versus freehand for in-/out-of-plane achieved 100.0/100.0 vs. $\quad 83.3 / 33.3 \%$ successfulness $(\mathrm{p}=0.478 / \mathrm{p}<0.001), \quad 1.0 \pm 0.0 / 1.0 \pm 0.0$ vs. $3.4 \pm 1.2 / 4.8 \pm 0.4$ needle manipulations $(\mathrm{p}<0.001 / \mathrm{p}<0.001), 2.6 \pm 0.9 / 2.7 \pm 0.9$ vs. $4.8 \pm 2.5 / 14.4 \pm 12.5 \mathrm{~mm}$ Euclidean error $(p=0.002 / p<0.001), 1.0 \pm 0.6 / 0.8 \pm 0.6$ vs. $2.0 \pm 1.5 / 1.7 \pm 0.4 \mathrm{~Gy} \cdot \mathrm{mm}$ total dose-length-product $(\mathrm{p}=0.043 / \mathrm{p}=0.001)$ and $7: 25 \pm 1: 34 / 7: 40 \pm 1: 33$ vs. $10: 45 \pm 3: 48 / 21: 15 \pm 4: 50$ minutes placement time $(\mathrm{p}=0.013 / \mathrm{p}<0.001)$.

Conclusions The system consistently enables user-independent successful needle placement in a single insertion, with smaller placement error, lower radiation dose and shorter placement time. 


\subsection{Introduction}

Minimally invasive biopsy and ablation of tumours in the abdomen and thorax are typically performed via a needle which is inserted percutaneously ('through the skin') using image-guidance to reach the target tissue. X-ray spiral CT-guided percutaneous needle placement is normally performed freehand. The performance of this method is user-dependent and multiple iterations of needle placement and CT scanning for placement verification are generally required before satisfactory placement is achieved. Each iteration increases tissue damage with accompanied risks, patient $\mathrm{X}$-ray exposure, procedure time and costs.

A variety of systems has been developed over the past decades to improve the procedure of CT-guided needle placement, ranging from simple passive aids to fully automated robots [72]. Exemplary systems that surpass the experimental stage include the laser guidance system Amedo LNS [216], the navigation system CAScination CAS-One [217], and the systems Perfint MAXIO [218] and iSYS Medizintechnik iSYS1 [219] which provide physical needle guidance. Despite the various attempted strategies and often technically sound solutions, widespread clinical adoption of any system is lacking. A novel system for CT-guided percutaneous needle placement in the thorax and abdomen, the DEMCON NPS, was developed to enable user-independent precise needle placement in a single insertion, and designed with a focus on clinical acceptability, applicability and usability (Fig. 6.1). The strategy of the system is to provide precise alignment of a needle guide with a user-specified target for physical support during a single manual needle insertion.

A pre-clinical phantom study is presented with the objective to validate the system, by ex vivo comparison of the performances of guided and freehand needle placement. The simulated use case is CT-guided percutaneous needle placement for microwave ablation (MWA) of liver tumours.

\subsection{Materials \& methods}

This section first discusses the freehand and guided needle placement methods, followed by the experiment setup of the phantom study.

\subsubsection{Procedure \& freehand needle placement}

As a starting point of the MWA procedure, the patient is accommodated on the CT table, general anaesthesia and medical ventilation are applied and a sterile workspace is created. An initial set of exploratory $\mathrm{CT}$ images of the relevant anatomical area is acquired and reviewed on a DICOM image viewer to find the tumour and plan a suited needle path. A radiocontrast agent may be administered to enhance tumour visibility. A sheet of radiopaque grid-lines is placed on the patient's skin around the estimated location of the needle entry point and 


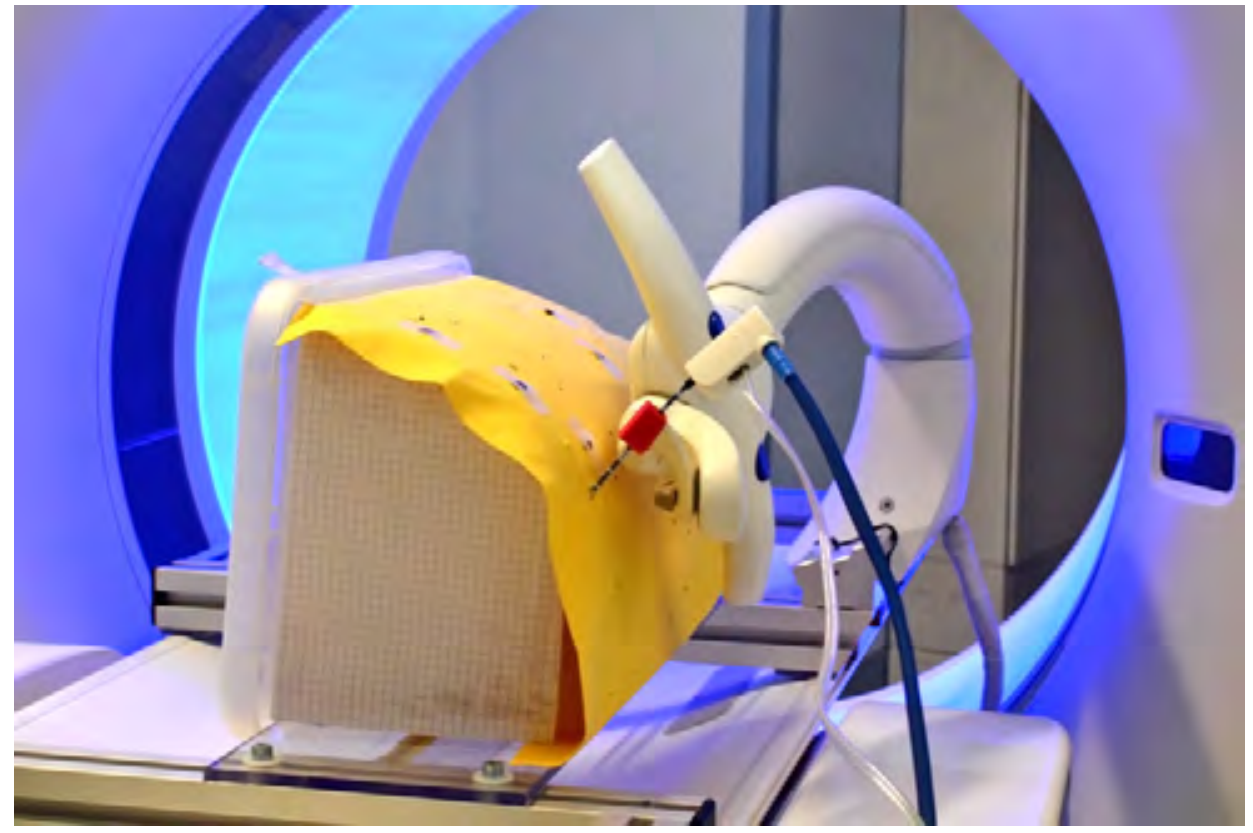

Figure 6.1: Prototype of the validated system for CT-guided needle placement during experimental validation.

a second CT scan is performed. The entry point is commonly chosen such that it coincides with one of the grid-lines. The patient table is translated such that the plane of a laser incorporated in the CT gantry coincides with the image position of the planned entry point. The crossing of the laser plane with the selected grid line marks the entry point on the patient's skin.

The next step is to estimate the path angles from entry point to target on the available CT images and insert the needle accordingly. An iterative method of freehand needle insertion and subsequent CT scanning for verification is employed. A lightweight hypodermic needle may be superficially inserted as a first estimate. The required insertion depth is determined on the DICOM image viewer and measured with respect to the skin using linear markings on the needle. All scanning sequences and insertions are performed under paused respiration in the same state to minimize deviations of the tumour position. Ablation is started once the needle tip has successfully reached the target. Freehand needle placement is particularly challenging for paths out-of-plane, i.e. oblique to the transversal CT image, which are common to reach tumours in the upper part of the liver without puncturing the lung. 


\subsubsection{Novel system \& guided needle placement}

The DEMCON NPS (Fig. 6.1) incorporates a needle guide which is aimed by a 2 degrees of freedom (2-DOFs) actuated remote centre of motion (RCM) mechanism, referred to as the orientation module $(\mathrm{OM})$. The $\mathrm{OM}$ includes handlebars and can freely be placed around the patient and locked with respect to the patient table via a 6-DOFs linkage, referred to as the locking module (LM).

The system is installed on the patient table prior to the procedure. A first CT scan of the relevant part of the anatomy is performed for initial path planning and entry point retrieval, identically to the freehand method (Fig. 6.2a). The OM is manually placed by the physician to coincide the RCM with the entry point and push-button locked to the table by the LM (Fig. 6.2b). The RCM is hereto indicated by a pointer placed in the needle guide. A second CT scan is performed of the relevant anatomy together with the OM (Fig. 6.2c), and the images are loaded into the system's graphical user interface (GUI). Four fiducial markers are incorporated in the OM, which serve as references for automated OM-to-CT registration [181]. The GUI includes a DICOM viewer used for target specification. The planned path from RCM to target is superimposed on the images for review. The required angles of the RCM mechanism to align the needle guide with the trajectory from RCM to target are automatically calculated, together with the corresponding insertion depth.

Upon approval of the planned path in the GUI, a button on the LM becomes active. When the button is pushed, the RCM mechanism automatically rotates to the required angles, aligning the needle guide with the target (Fig. 6.2d). A blue light-emitting diode (LED) on the LM turns on to indicate aiming is ready. The needle is now inserted manually in a single action through the needle guide to the specified depth (Fig. 6.2e). A third CT scan is performed for placement verification (Fig. 6.2e).

\subsubsection{Experiment setup}

Both needle placement methods were employed to approach targets in a static homogeneous gelatin phantom as an artificial substitute to the human abdominal anatomy, under controlled conditions for performance comparison. Two sets of 6 paths each were prepared by predefined target positions and corresponding entry points. The sets are distinguished by in-plane and $30^{\circ}$ out-of-plane paths. The nominal insertion depth from entry point to target for all paths in both sets was maintained at $120 \mathrm{~mm}$. The used needle was a $20 \mathrm{~cm}$ long, 13 gauge (Ø2.4 mm) Covidien [206] EMprint MWA needle with a 3-facet diamond shape tip. Two physicians were involved as operators in the experiment. Both operators routinely perform freehand CT-guided percutaneous needle placement and have at least six years of experience with this type of procedure. Each operator attempted each path by each method once in random order, yielding a total of $2 \times 12 \times 2=48$ attempted paths. 


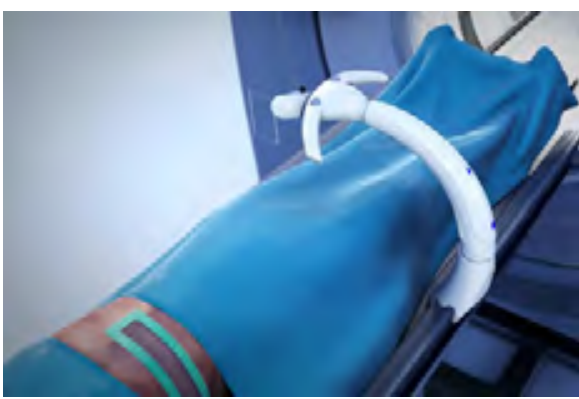

(a) The system installed on the patient table, awaiting initial path planning and entry point retrieval.

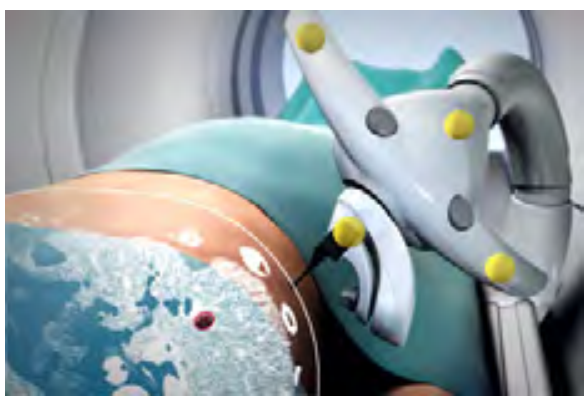

(c) CT scanning of patient and OM for OM-toCT registration using four fiducial markers and for target specification.

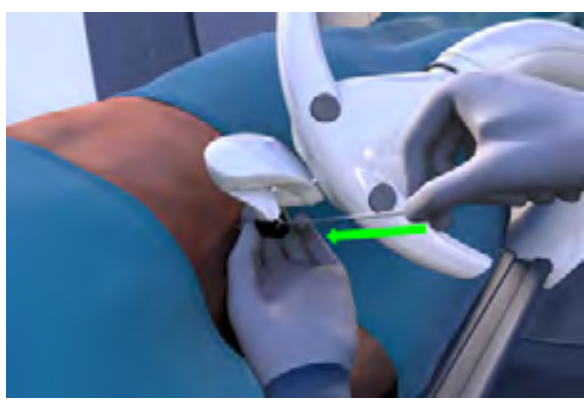

(e) Manual needle insertion through the needle guide to specified depth.

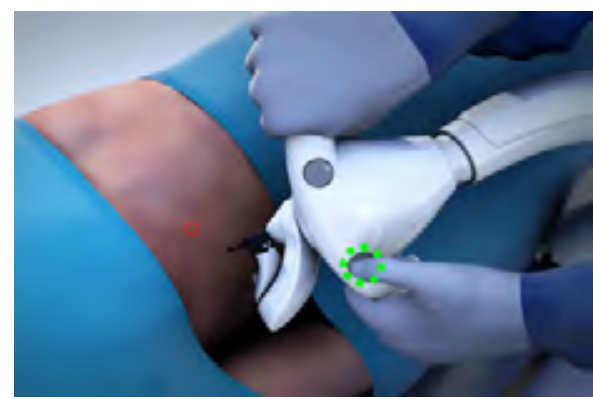

(b) Manual placement of the OM such that the RCM coincides with the entry point and pushbutton controllled locking to the patient table by the LM.

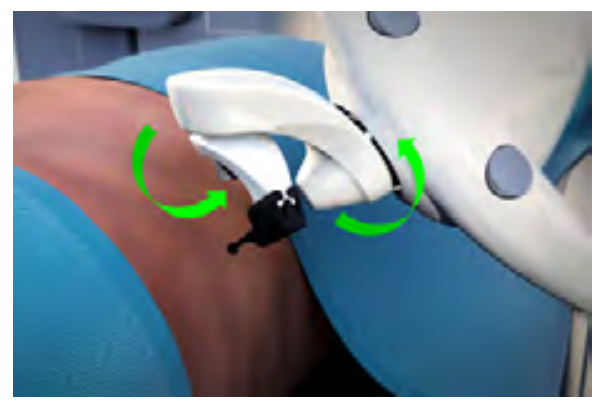

(d) Automated aiming of the needle guide by the RCM mechanism.

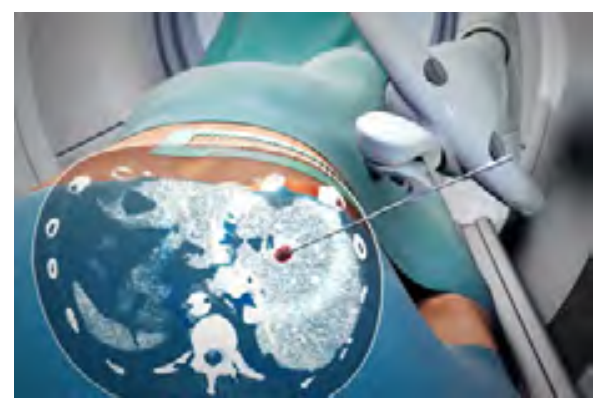

(f) CT scanning for placement verification.

Figure 6.2: Illustrations of the system's guided method for needle placement. 


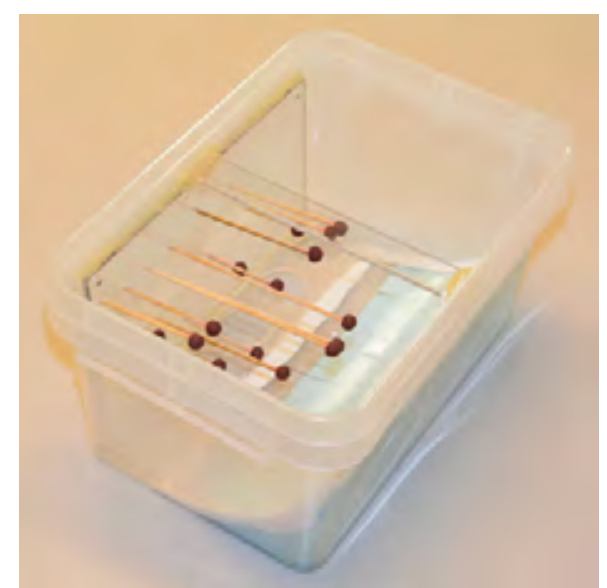

(a) Structural frame with targets on skewers, inside the box for moulding.

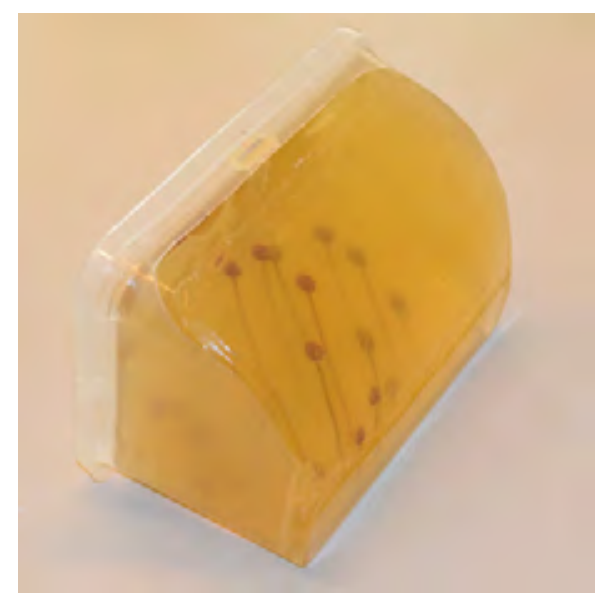

(b) Structural frame and cast gelatin after ejection from the mould box.

Figure 6.3: Abdominal phantom construction.

\section{Phantom construction}

The phantom incorporates a frame cut from a plastic box $(345 \times 230 \times 160 \mathrm{~mm})$ for structural integrity (Fig. 6.3). Glued to the inside of the frame is a $5 \mathrm{~mm}$ PMMA plate with a grid of holes, in which $\varnothing 2 \mathrm{~mm}$ wooden skewers are inserted. Clay spheres mixed with carbonyl iron powder for image contrast (10:100 powder to clay weight ratio) are placed at the tip of the skewers as targets. The frame fits into a second plastic box with a rounded bottom, which serves as a mould to cast the gelatin (15:100 gelatin powder to water weight ratio) (Fig. 6.3a). Once the gelatin has set, it is ejected with the frame from the outer box (Fig. 6.3b). The rounded gelatin surface represents the side of the abdomen, and is covered by synthetic chamois leather to block visibility of the targets. The position of the holes and length of the skewers position the targets such that the intended inplane and $30^{\circ}$ out-of-plane paths of $120 \mathrm{~mm}$ insertion depth are established from the corresponding entry points. The prepared paths are sufficiently far apart to avoid intersection of the needle with present tracts of previously attempted paths. A flexible plastic sheet with holes is used as a template to mark the predefined entry points on the chamois leather. Dots of the same clay/carbonyl iron powder mixture are placed on the entry points for indication on the images of the initial exploratory CT scan. The frame of the phantom was bolted onto a plexiglas tray, which was fixated via aluminium frames to the patient table (Fig. 6.1). The gelatin was recast after all prepared paths were attempted, such that repetition by an altered method or operator always started in intact gelatin. 


\section{System \& CT scanner integration}

The system was integrated with a Siemens Somatom Force CT scanner. Its linear rail was mounted on a provisional aluminium frame fixed to the patient table (Fig. 6.1). Scan and reconstruction protocols clinically used for minimally invasive tumour ablation were applied, with a constant $1.0 \mathrm{~mm}$ slice thickness. Upon image reconstruction, DICOM files were automatically and instantly transferred to the computer of the needle placement system using K-PACS [220] server software.

\section{Calibration of the needle placement system}

The relative positions of the fiducial markers and the functional geometry of the RCM mechanism were measured using a FARO [212] GagePlus measuring arm with a specified volumetric length measuring uncertainty of $5 \mu \mathrm{m}$. The 2-DOFs RCM mechanism relies on incremental rotatory encoders for positional feedback. A homing procedure is therefore performed, in which the RCM mechanism moves to mechanical end stops. The corresponding home angles were calibrated prior to the experiment by repeated targeting at $\varnothing 2 \mathrm{~mm}$ lead balls taped to the patient table at $\geqslant 250 \mathrm{~mm}$ from the RCM, and updating the segment home angles to align the tip of a Ø2.4 $\mathrm{mm}$ steel needle with the balls.

\section{Experiment execution}

Figure 6.4 shows a flowchart of the two methods as followed in the experiment. At the defined start of both methods, an initial exploratory set of CT images of the phantom is available, on which the predefined targets and corresponding entry points are clearly visible. Also, the entry points are marked on the chamois leather.

Needle placement was considered successful once the Euclidean ('point-topoint') error between needle tip and target was $\leqslant 5 \mathrm{~mm}$, which is deemed sufficient for the intended clinical application. A maximum of 5 manipulations of the MWA needle was permitted for the iterative freehand method. Placement of the auxiliary hypodermic needle in the freehand method was optional and could freely be skipped according to the operator's own judgement. Only 1 insertion was permitted for the guided method, in accordance with the intended use of the needle placement system. OM-to-CT registration is obstructed when the imaged volume does not encompass all four fiducial markers. In this case, either the placement of the OM, the table height, the field of view and/or the scan range were adjusted and a new scan was performed.

Measures of performance are the percentage of successful needle placements, the number of MWA needle insertions, the geometric placement errors, the number of CT scans, radiation dose, and procedure time from skin incision (in case of 


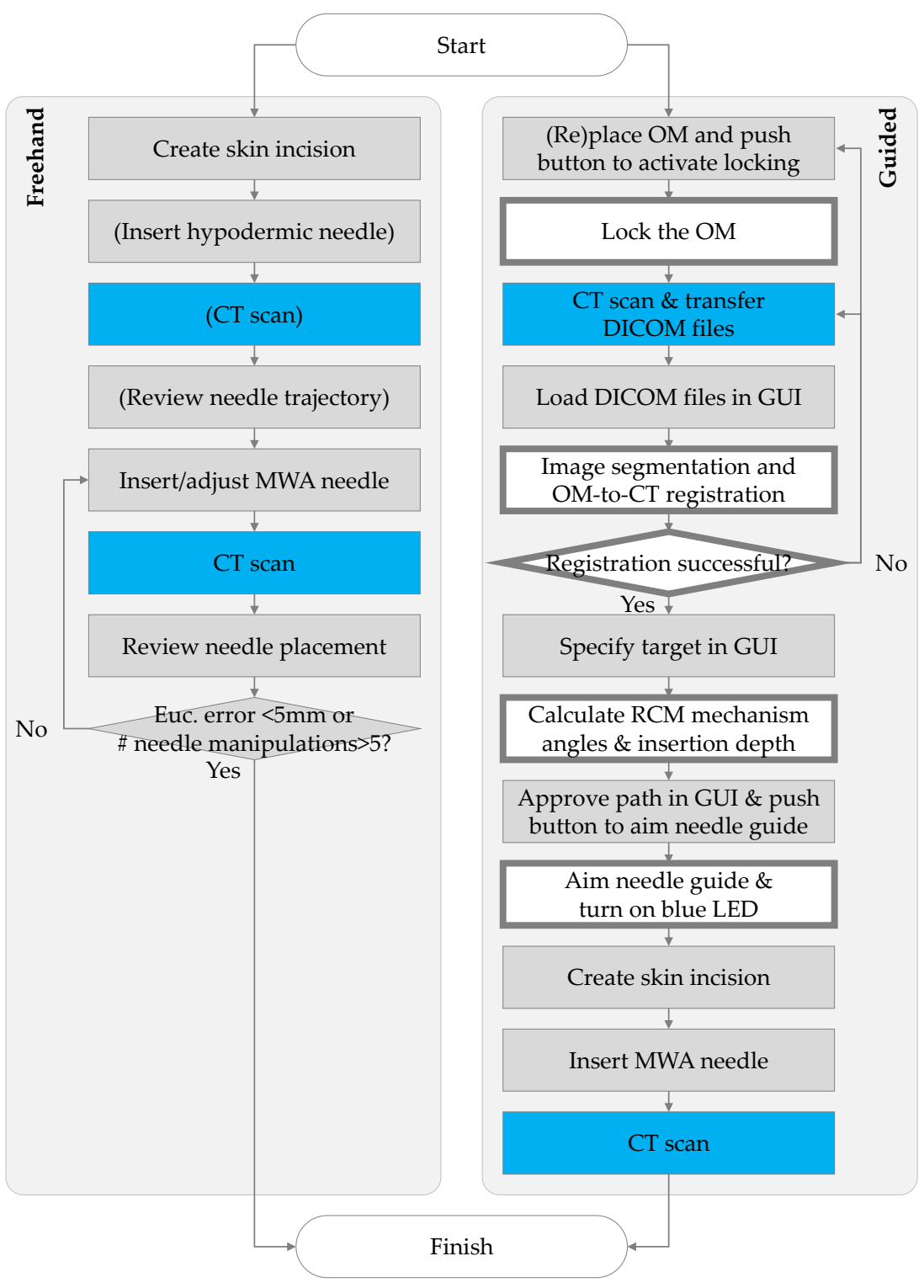

Figure 6.4: Flowchart of the freehand and guided needle placement methods.

the freehand method) or OM placement (in case of the guided method) to last CT scan.

Prior to the experiment execution, the functioning and use of the needle placement system, both placement methods and the experiment setup were thor- 


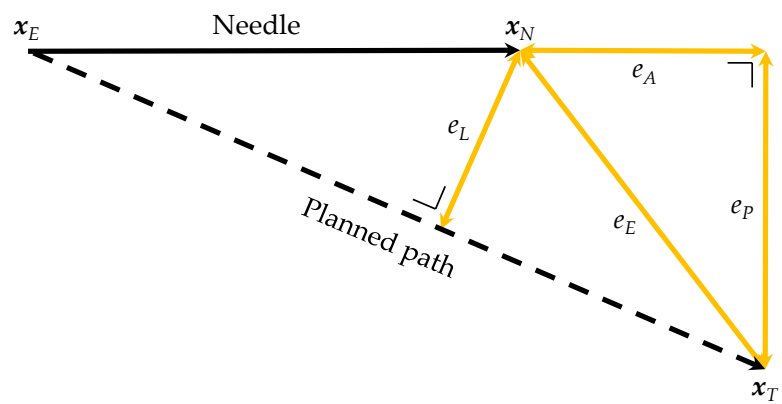

Figure 6.5: Illustration of the geometrical definition of the Euclidean $\left(e_{E}\right)$, lateral $\left(e_{L}\right)$, axial $\left(e_{A}\right)$ and projected error $\left(e_{P}\right)$.

oughly explained to the operators. Each operator performed three needle placements by each method as part of the preparation. Data from these trials are excluded from the results.

\section{Data acquisition \& processing}

The number of MWA needle manipulations were counted and the times of skin incisions, OM placements and CT scans were monitored on a digital clock. All DICOM files containing the reconstructed CT images and metadata were stored together with the CT coordinates of the target $\left(x_{T}\right)$, skin entry point $\left(x_{E}\right)$ and needle tip $\left(x_{N}\right)$ after each insertion. Four errors are geometrically defined by these coordinates (Fig. 6.5). The Euclidean error (the point-to-point distance between needle tip and target):

$$
e_{E}=\left\|\mathbf{x}_{N}-\mathbf{x}_{T}\right\|,
$$

the lateral error (the maximum distance between needle tip and planned path):

$$
e_{L}=\frac{\left\|\left(\mathbf{x}_{N}-\mathbf{x}_{E}\right) \times\left(\mathbf{x}_{N}-\mathbf{x}_{T}\right)\right\|}{\left\|\mathbf{x}_{T}-\mathbf{x}_{E}\right\|},
$$

the axial error (the distance along the needle from its tip to the point closest to the target):

$$
e_{A}=\left\|\frac{\left(\mathbf{x}_{T}-\mathbf{x}_{E}\right) \cdot\left(\mathbf{x}_{N}-\mathbf{x}_{E}\right)}{\left\|\mathbf{x}_{N}-\mathbf{x}_{E}\right\|}-\right\| \mathbf{x}_{N}-\mathbf{x}_{E}\|\|,
$$

and the projected error (the minimum distance between target and needle path):

$$
e_{P}=\frac{\left\|\left(\mathbf{x}_{T}-\mathbf{x}_{E}\right) \times\left(\mathbf{x}_{T}-\mathbf{x}_{N}\right)\right\|}{\left\|\mathbf{x}_{N}-\mathbf{x}_{E}\right\|} .
$$


The dose-length product (DLP) was calculated for each scan as a measure for radiation dose [221], by summation of the product of the volume CT dose index $C T D I_{\text {vol }}(i)$ and slice spacing $I$ for each image $i$ in $n$ images:

$$
D L P=\sum_{i=1}^{n} C T D I_{\text {vol }}(i) \cdot I .
$$

The total DLP was calculated by summation of the DLP of the individual scans used per approached target.

\section{Statistical data analysis}

The statistical significance of differences in successfulness between the groups were compared using chi-square tests. The remaining parameters were first checked for normality using the Shapiro-Wilk test. Subsequently, independent samples t-tests were performed in case of a normal distribution or WilcoxonMann-Whitney tests in case of a non-normal distribution. All statistical tests were conducted in IBM [222] SPSS Statistics, version 23. Differences were considered statistically significant for $\mathrm{p}<0.05$.

\subsection{Results}

The flowchart of Fig. 6.4 was completed by both operators for all paths using both the freehand and guided method (Fig. 6.6 and 6.7). Table 6.1 provides a summary of the results per path type and method, and combined for both operators. Complementing the listed errors is a specification whether the minimum, maximum or final (i.e. last) error value of subsequent needle manipulations for an approached target is considered.

The guided method achieved 100.0/100.0\% successfulness versus $83.3 / 33.3 \%$ $(\mathrm{p}=0.478 / \mathrm{p}<0.001)$ for the freehand method for in-/out-of-plane paths respectively. All targets were successfully reached in a single insertion by the guided method, whereas the freehand method used a mean of $3.4 \pm 1.2 / 4.8 \pm 0.4$ $(\mathrm{p}<0.001 / \mathrm{p}<0.001)$ needle manipulations, truncated by a maximum of 5 per approached target.

Mean final Euclidean errors of $2.6 \pm 0.9 / 2.7 \pm 0.9 \mathrm{~mm}$ were achieved by the guided method for in-/out-of-plane paths respectively, versus $4.8 \pm 2.5 / 14.4 \pm 12.5$ $\mathrm{mm}(\mathrm{p}=0.002 / \mathrm{p}<0.001)$ by the freehand method. The mean minimum value of the Euclidean error (the smallest achieved distance between tip and target) is lower than its mean final value for the freehand method, at $4.6 \pm 1.9 / 9.5 \pm 4.9 \mathrm{~mm}$, since the Euclidean error would occasionally increase rather than decrease for a subsequent needle manipulation. Mean maximum lateral errors (the maximum deviation of the needle tip off the planned path) of $1.6 \pm 0.7 / 2.3 \pm 1.2 \mathrm{~mm}$ were found for the guided method, versus $10.8 \pm 6.2 / 20.5 \pm 11.5 \mathrm{~mm}(\mathrm{p}<0.001 / \mathrm{p}<0.001)$ for the 


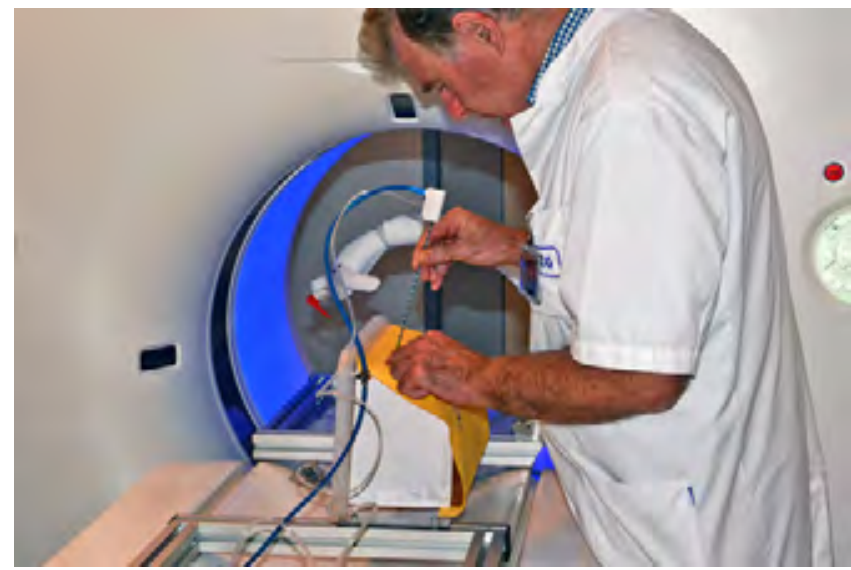

(a) Freehand manual needle insertion.

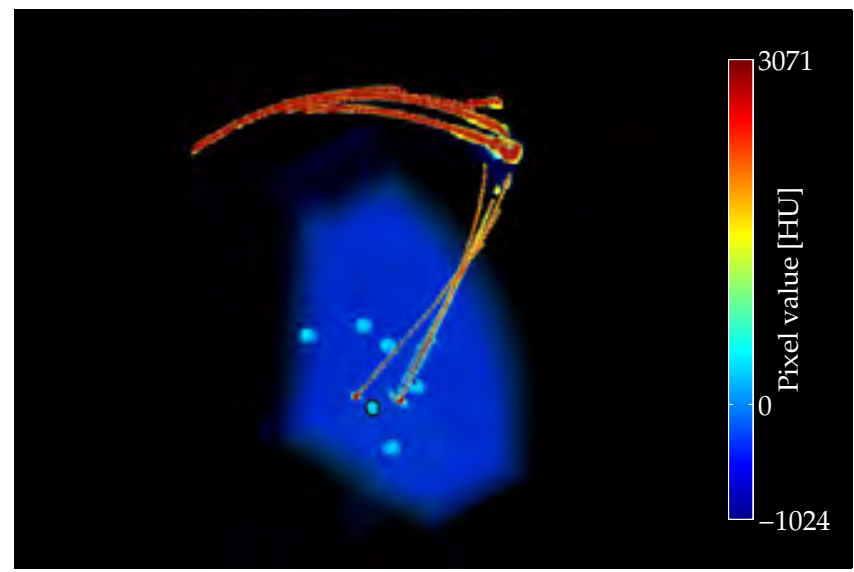

(b) 3D CT image of 6 superimposed scans for verification of subsequent attempts to reach the outlined target.

Figure 6.6: Experiment execution using the freehand method.

freehand method. No major difference per method was found for the final axial error for in-plane paths: $1.7 \pm 1.2 \mathrm{~mm}$ for guided versus $1.7 \pm 1.8 \mathrm{~mm}(p=0.564)$ for freehand. For out-of-plane paths, the final axial error of the guided method was slightly better than freehand, at $1.0 \pm 0.7 \mathrm{~mm}$ versus $2.9 \pm 2.1 \mathrm{~mm}(\mathrm{p}=0.011)$ respectively. Final projected errors of $1.6 \pm 0.7 / 2.3 \pm 1.2 \mathrm{~mm}$ were achieved by the guided method for in-/out-of-plane paths respectively, versus $4.3 \pm 2.1 / 13.9 \pm 12.5$ $\mathrm{mm}(\mathrm{p}<0.001 / \mathrm{p}<0.001)$ by the freehand method. The iterative character and userdependency of the freehand method is illustrated by plotting the lateral error versus insertion depth (Fig. 6.8) and the projected error versus the needle inser- 


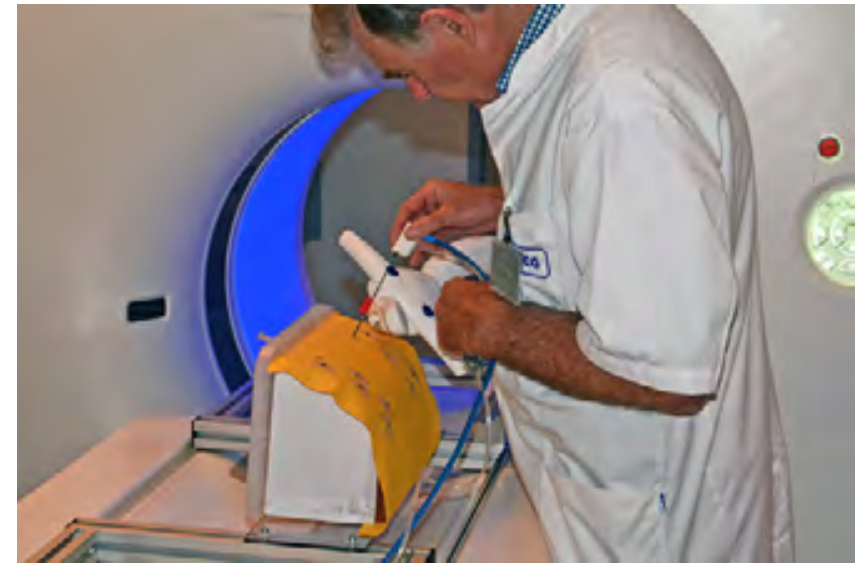

(a) Guided manual needle insertion after automatic aiming.

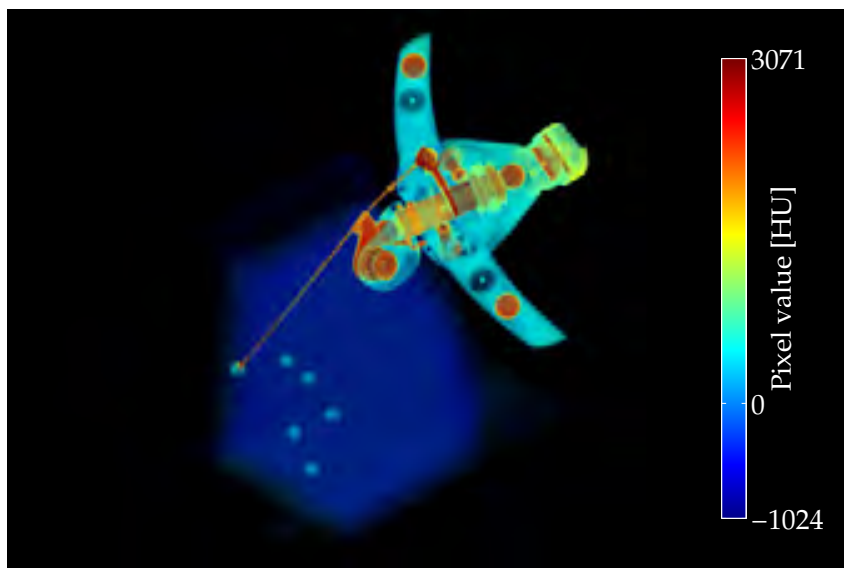

(b) 3D CT image for verification, confirming successful needle placement.

Figure 6.7: Experiment execution using the guided method.

tion number (Fig. 6.9). Initial estimates of the needle direction deviate up to 35/50 $\mathrm{mm}$ off target for in-/out-of-plane paths. The projected errors tend to improve with each subsequent manipulation for in-plane paths. Less improvement and a larger difference between the two operators was found for out-of-plane paths. 


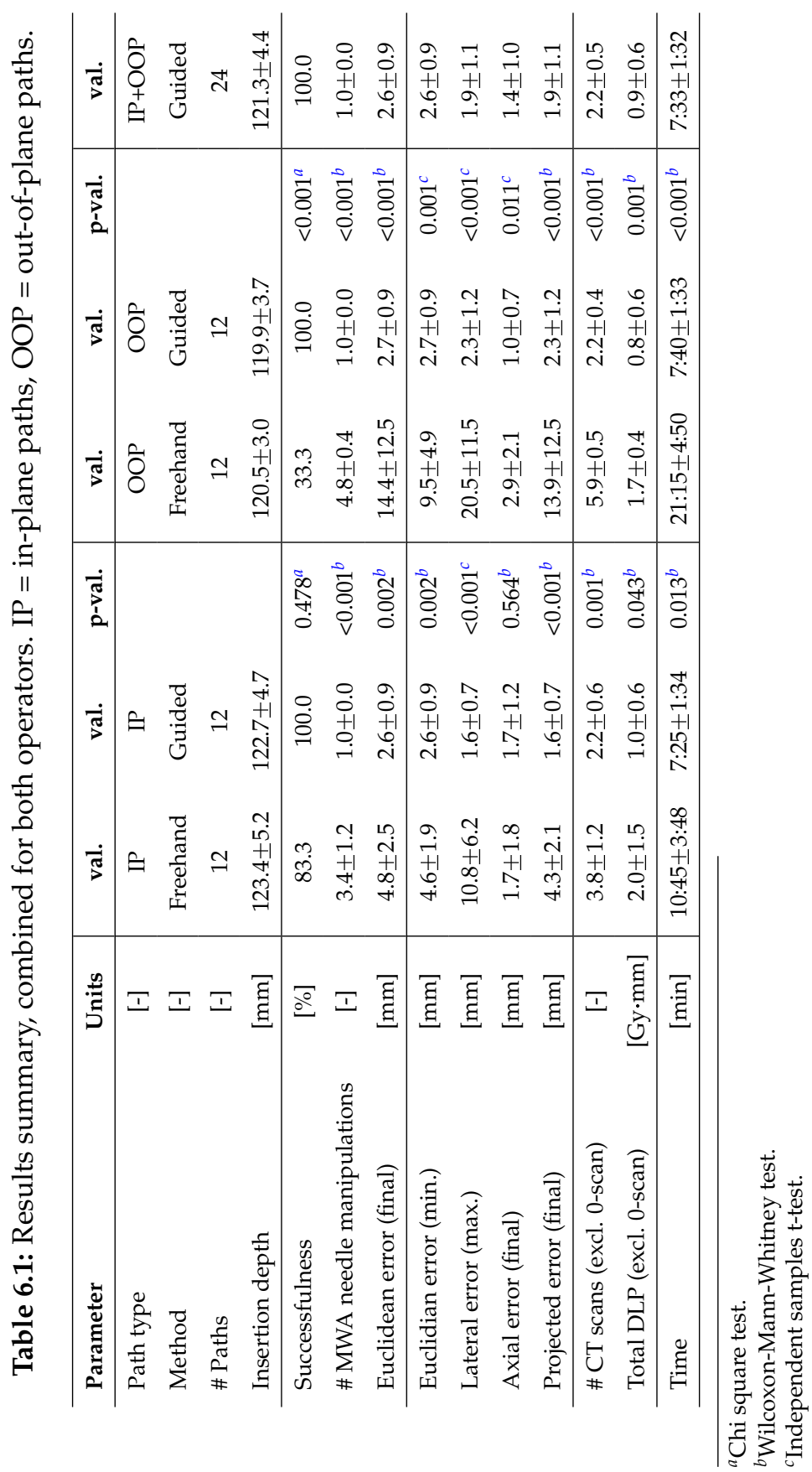




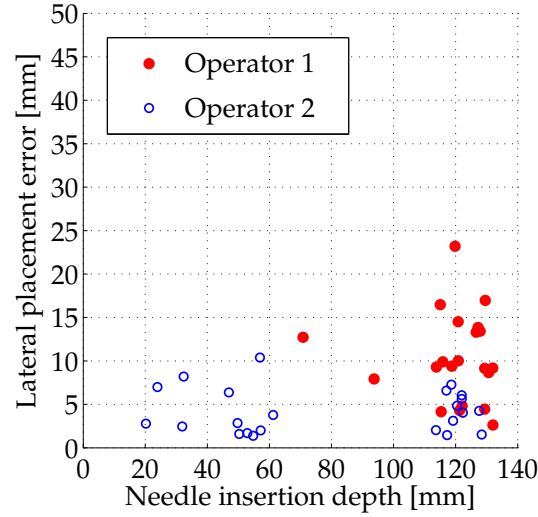

(a) In-plane paths.

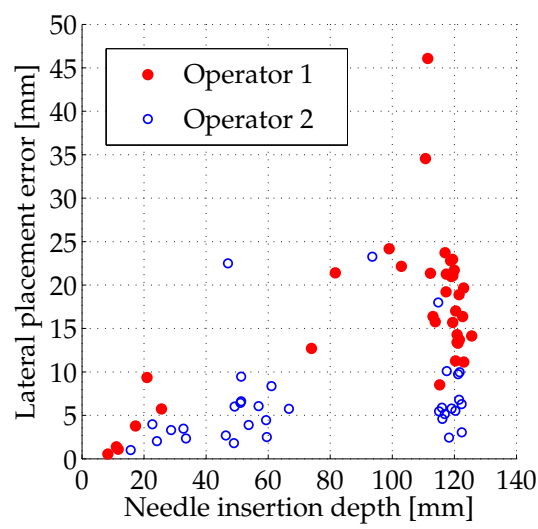

(b) Out-of-plane paths.

Figure 6.8: Lateral error versus insertion depth for the freehand method.

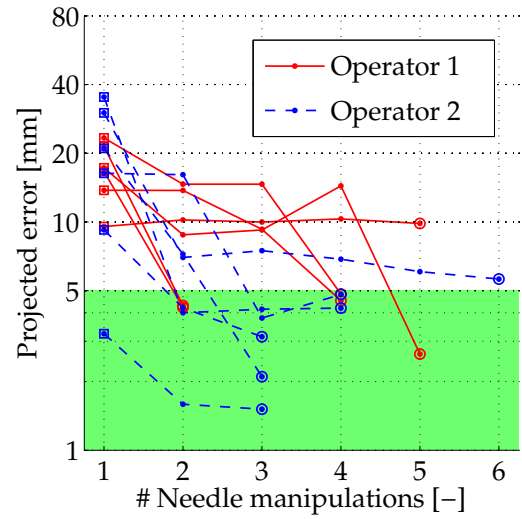

(a) In-plane paths.

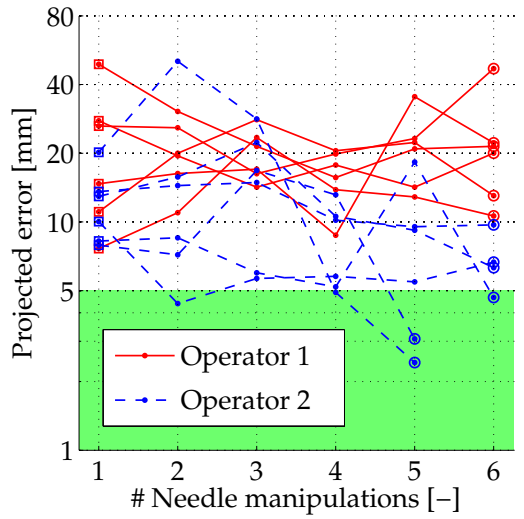

(b) Out-of-plane paths.

Figure 6.9: Projected error versus number of needle manipulations (of the MWA needle and the hypodermic needle if applicable) with intermediate CT-scans for the freehand method.

The guided method required 2.2 $\pm 0.6 / 2.2 \pm 0.4 \mathrm{CT}$ scans (excluding the initial 0 -scan) for in-/out-of-plane paths respectively, versus $3.8 \pm 1.2 / 5.9 \pm 0.5$ $(\mathrm{p}=0.001 / \mathrm{p}<0.001)$ for the freehand method. OM-to-CT registration was ob- 


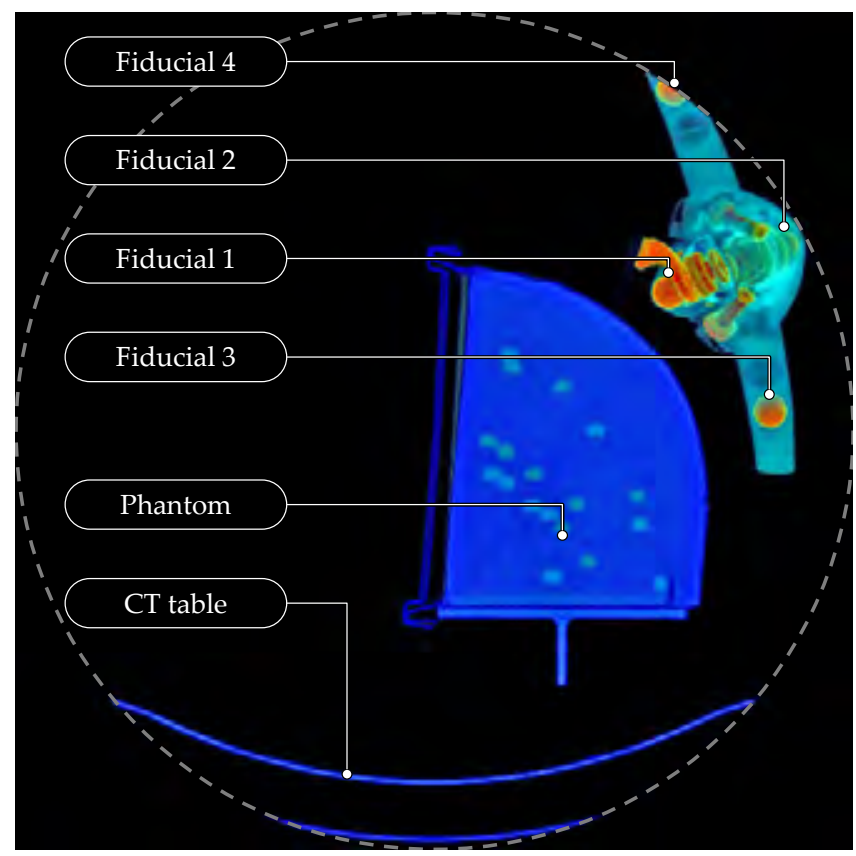

Figure 6.10: Front view of a 3D CT image in which segmentation was obstructed due to partial exclusion of fiducial 4 from the imaged volume.

structed in 4 cases when the imaged volume did not encompass all four fiducial markers (Fig. 6.10) and a new scan was required. The total DLP used by the guided method (excluding the initial 0 -scan) were $1.0 \pm 0.6 / 0.8 \pm 0.6 \mathrm{~Gy} \cdot \mathrm{mm}$, versus $2.0 \pm 1.5 / 1.7 \pm 0.4 \mathrm{~Gy} \cdot \mathrm{mm}(\mathrm{p}=0.043 / \mathrm{p}=0.001)$ for the freehand method.

Time from OM placement to last CT scan for the guided method measured 7:25 $\pm 1: 34 \mathrm{~min}$ and 7:40 $\pm 1: 33 \mathrm{~min}$ for in- and out-of-plane paths respectively. The freehand method took significantly longer, at 10:45 $\pm 3: 48 \mathrm{~min}(\mathrm{p}=0.013)$ and 21:15 $\pm 4: 50 \mathrm{~min}(\mathrm{p}<0.001)$ respectively from skin incision to last CT scan.

The right column of Table 6.1 lists the performance of the guided method combined for both in-plane and out-of plane paths: $100.0 \%$ successful needle placement was achieved in a single insertion, with $2.6 \pm 0.9 \mathrm{~mm}$ Euclidean error, $2.2 \pm 0.5 \mathrm{~Gy} \cdot \mathrm{mm}$ total DLP and a placement time of 7:33 $\pm 1: 32$.

\subsection{Discussion}

A novel system for CT-guided percutaneous needle placement has been validated by a performance comparison of its guided method and the conventional freehand method in a phantom study, simulating a use case of needle placement 
for MWA of liver tumours. Two operators each employed both methods to reach targets at $120 \mathrm{~mm}$ depth via in-plane and $30^{\circ}$ out-of plane paths in a static and homogeneous gelatin phantom, with a defined goal to reach $\leqslant 5 \mathrm{~mm}$ Euclidean error in $\leqslant 5$ MWA needle manipulations.

The guided method achieved $100.0 \%$ successfulness, superior with statistical significance to the $33.3 \%$ of the freehand method for out-of-plane paths. No significant difference was found with the $83.3 \%$ successfulness of the freehand method for in-plane paths. The guided method consistently reached successful placement in a single insertion, versus $3.4 \pm 1.2 / 4.8 \pm 0.4$ needle manipulations by the freehand method for in-/out-of-plane paths respectively, truncated by a maximum of 5 per approached target. A common difficulty of the iterative freehand method also encountered in clinical practise is the tendency of the MWA needle to follow the trail left behind by a previous off-target insertion. The guided method avoids this problem by precise alignment of the needle guide prior to a single insertion.

Significant superiority of the guided method was also found in terms of final Euclidean error, number of CT scans, total DLP and needle placement time, for both in- and out-of-plane paths. For the freehand method, the total DLP for outof-plane paths is lower than for in-plane paths, despite a larger number of CT scans. This is attributed to a large dependency of the DLP on the size of the imaged volume selected manually by the operator of the CT scanner. Additional time for system installation in the intervention suite and application of a sterile cover were not included in this validation. In consideration, the system could be installed once for multiple subsequently scheduled procedures and application of a sterile cover could be performed parallel to other preparations, such that these do not necessarily delay the procedure, in contrast to time required for needle placement.

A major and significant difference was found in terms of maximum lateral error, the maximum distance of the needle tip off the path from entry point to target. The values of $10.8 \pm 6.2 / 20.5 \pm 11.5 \mathrm{~mm}$ for in-/out-of-plane freehand needle placement define the radius of an uncertainty zone around the needle path to take into account during path planning. The system provides a reduction of this zone to only $1.6 \pm 0.7 / 2.3 \pm 1.2 \mathrm{~mm}$, which could improve accessibility of targets via paths passing critical structures which should not be punctured.

Table 6.2 lists four alternative systems and the DEMCON NPS for comparison of the achieved Euclidean errors at specified insertion depths in static phantoms. The Amedo LNS [216] is a laser guidance system for freehand needle placement, limited to in-plane paths. The contactless guidance advantageously avoids the introduction of any added sterile or covered components, but the achieved error of $4.0 \pm 2.7 \mathrm{~mm}$ at $94.2 \pm 16.9 \mathrm{~mm}$ depth is inferior to that of the DEMCON NPS. The CAScination CAS-One [217] is an optical tracking based navigation system. It assists freehand needle placement by providing a real-time graphical visualization of the manipulated needle with respect to a static image set by tracking of fiducial markers attached to the needle. An error of $4.9 \pm 1.7 \mathrm{~mm}$ at $103.6 \pm 18.1 \mathrm{~mm}$ depth was reported, comparable to the Amedo LNS. The required number of needle 
Table 6.2: Performance comparison of systems for CT-guided needle placement.

\begin{tabular}{lcc}
\hline System & $\begin{array}{c}\text { Insertion depth } \\
{[\mathrm{mm}]}\end{array}$ & $\begin{array}{c}\text { Euclidean error } \\
{[\mathrm{mm}]}\end{array}$ \\
\hline Amedo LNS [216] & $94.2 \pm 16.9$ & $4.0 \pm 2.7$ \\
CAScination CAS-One [217] & $103.6 \pm 18.1$ & $4.9 \pm 1.7$ \\
Perfint MAXIO [218] & $110 \pm 38$ & $6.5 \pm 2.5$ \\
iSYS Medizintechnik iSYS1 [219] & $92.8 \pm 14.4$ & $2.3 \pm 0.8$ \\
DEMCON NPS & $121.3 \pm 4.4$ & $2.6 \pm 0.9$ \\
\hline
\end{tabular}

manipulations and CT scans to achieve successful placement was not reported in [216] nor [217]. The Perfint MAXIO [218] and iSYS Medizintechnik iSYS1 [219] employ the same strategy as the DEMCON NPS to align a needle guide with a user-specified target for physical support during a single manual needle insertion, but use different system architectures. The Perfint MAXIO is a large floor mounted system next to the CT table, providing large stroke 5-DOFs automated placement of a needle guide around the patient for manual insertion. The reported error of $6.5 \pm 2.5 \mathrm{~mm}$ at $110 \pm 38 \mathrm{~mm}$ depth is inferior to that of the DEMCON NPS. The iSYS Medizintechnik iSYS1 is a table mounted system providing 4-DOFs automated alignment of the needle guide after manual placement and locking at the site of insertion. The reported $2.3 \pm 0.8 \mathrm{~mm}$ error at $92.8 \pm 14.4 \mathrm{~mm}$ depth is comparable to that of the DEMCON NPS.

In conclusion, the presented system validation evidences the added value of the DEMCON NPS for needle placement under the controlled conditions of the phantom study and sets high expectations for its potential in clinical practise. It endorses the system's strategy to provide precise needle guidance to reach a target in a single manual insertion, its architectural design for usability of a manually placeable and push-button lockable RCM mechanism, automatic OM-to-CT registration using fiducial markers, and automatic aiming of the needle guide, and its detail design as successfully implemented in a fully functional prototype. 


\section{CHAPTER 7}

\section{Conclusions \& outlook}

\subsection{Conclusions}

The objective of the research described in this dissertation as formulated in chapter 1 has been:

the development of a precision system for image-guided percutaneous needle placement in the thorax and abdomen

In fulfilment of the objective, a clinically acceptable, widely applicable and usable system has been developed which enables user-independent, precise CT-guided needle placement in a single insertion, using a lower radiation dose and shorter placement time (Fig. 7.1).

As a starting point of the research, an overview was created of the state of the art of systems for CT- and MRI-guided percutaneous needle placement in the thorax and abdomen (chapter 2). Over forty systems were identified, ranging from simple passive aids to fully automated robots. Despite the various attempted strategies and often technically sound solutions, widespread clinical adoption of any system is lacking. None of the systems represent a breakthrough in percutaneous procedures on par with that of the imaging technology that made them possible, leaving open space for innovation.

A problem analysis of the existing clinical workflow and freehand method of CT-guided percutaneous needle placement at multiple medical centres indicated the prime opportunity for improvement lies in the current method of reaching a target by iterative needle placement and intermediate imaging for verification. Reducing the number of placement iterations needed to achieve satisfactory placement would be accompanied by a reduction in tissue damage, patient X-ray exposure, procedure time and costs. The strategy of a novel system for CT-guided percutaneous needle placement has been proposed, in which precise axial alignment of a needle guide is provided to reach a user-specified target in a single manual insertion (chapter 3).

Based on user requirements, the architectural and detail design of the system has been elaborated, outlining the revised workflow and placement method. The system consists of an orientation module (OM) and a locking module (LM). 


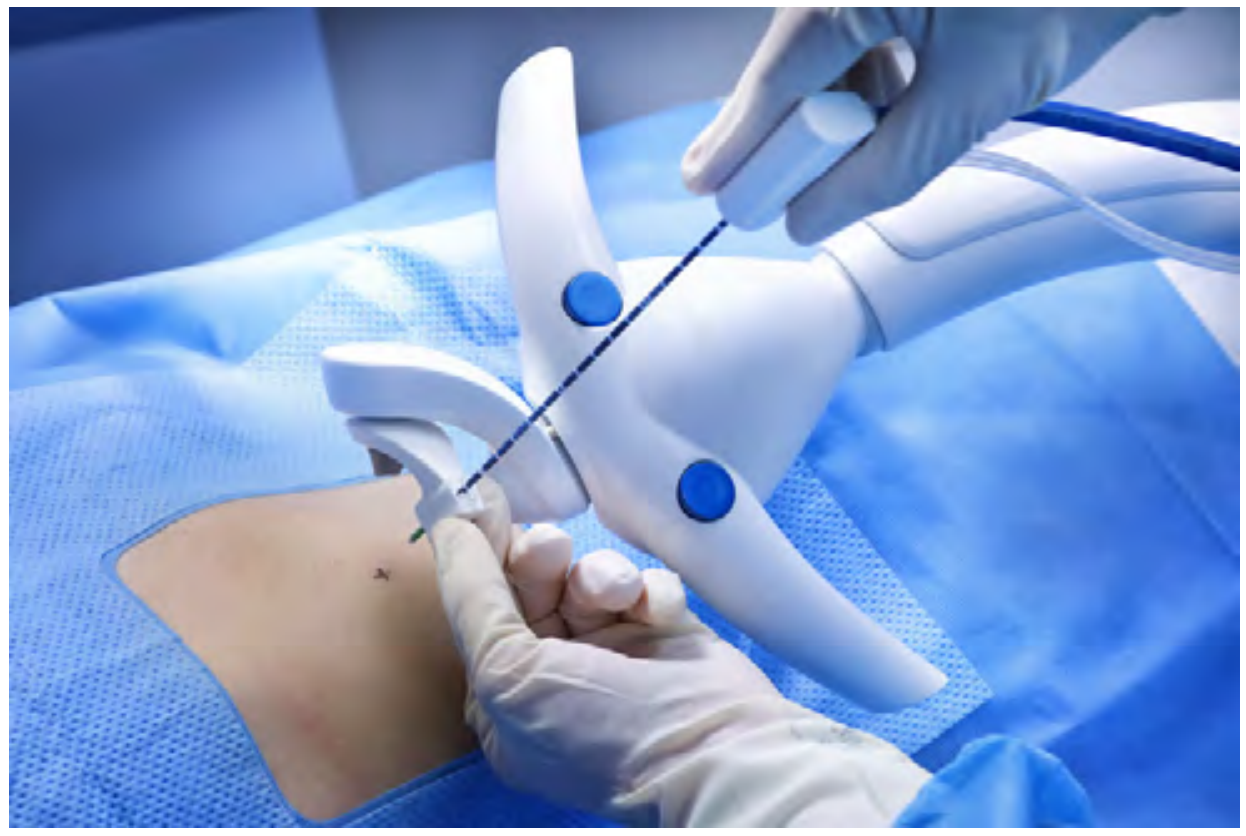

Figure 7.1: The developed system in the intervention suite.

The OM contains an automated two degrees of freedom (2-DOFs) mechanism to aim a needle guide about a remote centre of motion (RCM). The LM is a passive lockable 6-DOFs linkage connecting the OM to the CT table. The LM enables a physician to manually place the OM anywhere around the patient to coincide the RCM with the desired skin entry point and push-button lock it. The relevant anatomy of the patient and the OM are CT scanned to acquire a 3D image, for automatic OM-to-CT registration using fiducial markers incorporated in the $\mathrm{OM}$, and for target specification and path approval in a graphical user interface. The needle guide is automatically aimed at the target, which is then reached by a single manual needle insertion to indicated depth.

The prime consideration towards clinical acceptability of the system is to leave the simple yet responsible task of needle insertion in the hands of the physician. Precautions to maintain a sterile workspace are implemented and the development of the system and its design are in compliance with applicable standards. A wide clinical applicability is created by the freedom of placement of the OM around the patient, the angular range of the RCM mechanism to aim the needle guide and its range of compatible needle diameters. This enables accessibility of most body sites via in- or out-of-plane paths and extends the potential applicability beyond the original use case of biopsy and ablation in the liver and lungs. In consideration of usability, the clinical workflow is maintained largely identical 
except for the elimination of iterative steps. The challenging task of needle aiming is taken over by automation and rendered user-independent. OM-to-CT registration and calculations of segment angles and insertion depth are handled by software, and locking and aiming are automated and push-button controlled. The substitutive user effort due to introduction of the system consists of its initial installation in the intervention suite, pre-procedural component sterilization and application of a sterile cover.

A first prototype of the system has been realized and integrated in the intervention suite. Its functionality has been demonstrated for 24 in- and out-ofplane punctures in a static gelatin phantom. Placement errors of $1.2 \pm 0.6 \mathrm{~mm}$ and $2.1 \pm 0.7 \mathrm{~mm}$ at $79.0 \pm 8.4 \mathrm{~mm}$ and $156.0 \pm 6.9 \mathrm{~mm}$ depth respectively were achieved in a single insertion, in compliance with a clinical user requirement of $\leqslant 5 \mathrm{~mm}$ error. Given a retrieved entry point, each target was consistently reached using a first CT scan for OM-to-CT registration after placement of the OM and a second $\mathrm{CT}$ scan for placement verification after needle insertion.

In assessment of the strategy of the novel system, three contributors to the overall needle placement error were identified. Firstly, the alignment errors of the needle guide with respect to the specified target coordinates, which defined the functional requirements that directed the elaboration of the architectural and detail design of the novel system. Secondly, displacement of the target tissue due to patient movement or respiration, which is effectively minimized in current clinical practise by patient stabilization using cushioning and breath holds during image acquisition and needle insertion in the same respiratory phase. Thirdly, needle-tissue interaction, causing deflection of the needle tip and displacement of the target tissue. The deflections of a selection of clinically used biopsy and ablation needles with asymmetrical bevel and symmetrical Franseen tips have been quantified for insertions in ex vivo porcine liver tissue (chapter 4). For a symmetrical Franseen tip needle, a deflection off the straight trajectory of $2.0 \pm 0.6 \mathrm{~mm}$ at $120 \mathrm{~mm}$ insertion depth was measured. Larger mean deflections were measured for two axi-asymmetric bevel tip needles, at $9.2 \pm 2.4$ and $9.6 \pm 1.5 \mathrm{~mm}$ at 120 $\mathrm{mm}$ insertion depth. The behaviour of a bevel tip needle to deflect in direction of the bevel is advantageously used in conventional freehand needle placement to adjust an off-target needle trajectory by altering its axial orientation. However, given the availability of a precisely aligned needle guide with the intention to reach a target with $\leqslant 5 \mathrm{~mm}$ error in a single manual insertion without intermediate imaging, Franseen tip needles which follow a straight path are favourable, and bevel tip needles should be avoided.

The detail design of the novel system has been assessed from a technical point of view and its performance in terms of needle placement error in air has been analysed (chapter 5). The contributions of three prime subsystems to the placement error and that of the overall system have been individually quantified. For targets at $250 \mathrm{~mm}$ depth, errors of $<1.2 \mathrm{~mm}$ and $<3.3 \mathrm{~mm}$ were determined for the drive system of segment 1 and 2 respectively, $0.81 \pm 0.34 \mathrm{~mm}$ for the OM-to-CT registration system, and $0.35 \mathrm{~mm}$ and $0.43 \mathrm{~mm}$ for two realistic load cases of the 
structural link from OM and needle guide to CT table. The placement errors in air of the overall unloaded system measured $1.0 \pm 0.25$ and $2.6 \pm 0.7 \mathrm{~mm}$ at 100 and $250 \mathrm{~mm}$ target depth respectively, and do not exceed the $\leqslant 5 \mathrm{~mm}$ error specified as a clinical user requirement for needle placement in tissue.

The system has been validated by a performance comparison of its guided method with the conventional freehand method for CT-guided needle placement, simulating a use case of microwave ablation (MWA) of liver tumours. Two experienced physicians employed both methods to reach a total of 48 targets in a static gelatin phantom with $\leqslant 5 \mathrm{~mm}$ error in $\leqslant 5$ needle manipulations using an MWA needle, via predefined in-plane and $30^{\circ}$ out-of-plane paths and an insertion depth of $120 \mathrm{~mm}$ (chapter 6). A successfulness of $100.0 \%$ was achieved using the guided method, versus $83.3 \%(p=0.478)$ and $33.3 \%(p<0.001)$ using the freehand method for in-plane and out-of plane respectively. The performance of the guided method has been proven superior to the freehand method in every aspect with statistical significance $(\mathrm{p}<0.05)$ : successful needle placement was consistently achieved in a single insertion, with higher accuracy and precision, fewer CT scans, lower radiation dose and shorter placement time, evidencing the added value of the novel system.

In conclusion, the key output of this research is a fully functional precision system for CT-guided percutaneous needle placement in the thorax and abdomen, ready for deployment in current clinical practise. 


\subsection{Outlook}

Derived from its prime objective, the research described in this dissertation encompasses several additional developments and system applications.

\subsubsection{System integration with cone beam CT}

System integration with cone beam CT (CBCT) has been initiated. Functional feasibility was demonstrated for simulated lumbar punctures in a clay spinal phantom using a Philips [85] Allura Xper cone beam CT system (Fig. 7.2), in collaboration with Philips Healthcare [223]. Additional pockets are available in the handlebars of the prototype (Fig. 5.2) to accommodate a more compact constellation of fiducial markers in consideration of the smaller diameter of the field of view of CBCT, at the cost of a minor increase in target registration error.

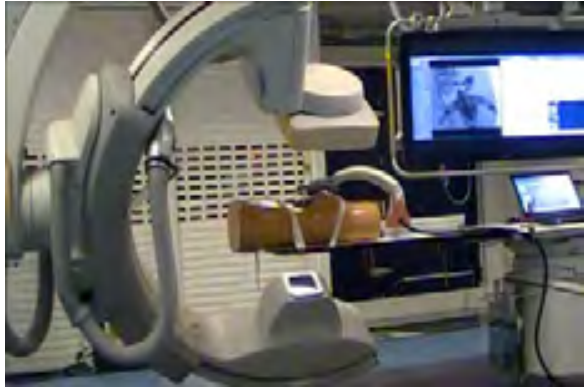

(a) CBCT scanning of the system and a spinal phantom.

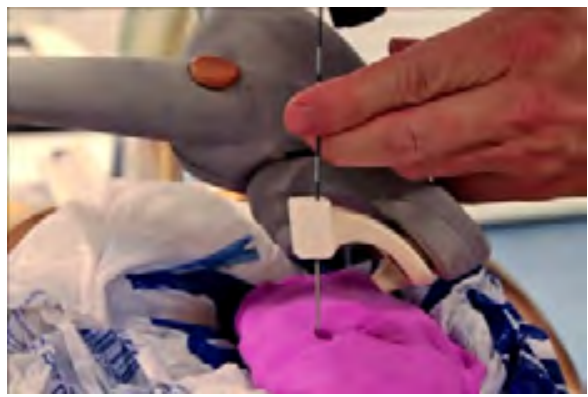

(c) Manual needle insertion after automatic aiming of the needle guide at the specified target.

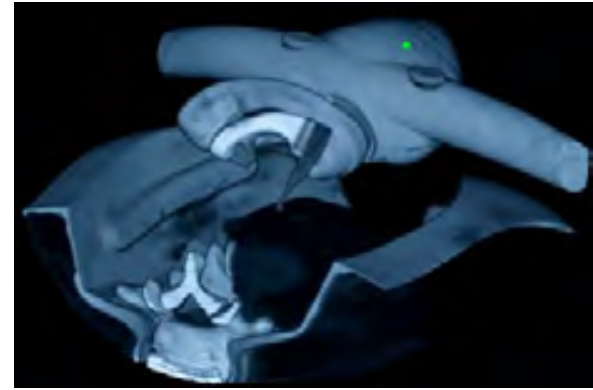

(b) 3D CBCT image used for OM-to-CBCT registration and target specification.

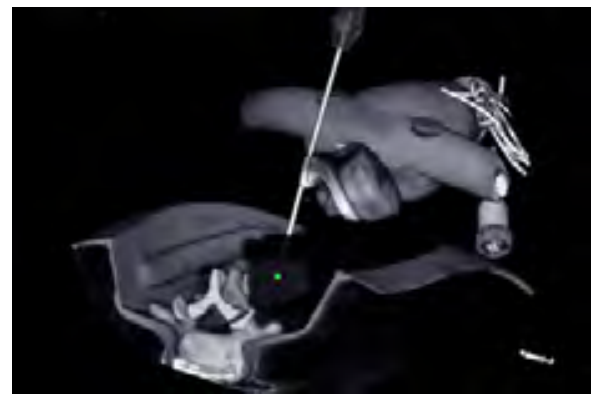

(d) 3D CBCT image after insertion for placement verification.

Figure 7.2: System integration with $\mathrm{CBCT}$ at the facilities of Philips Healthcare. 


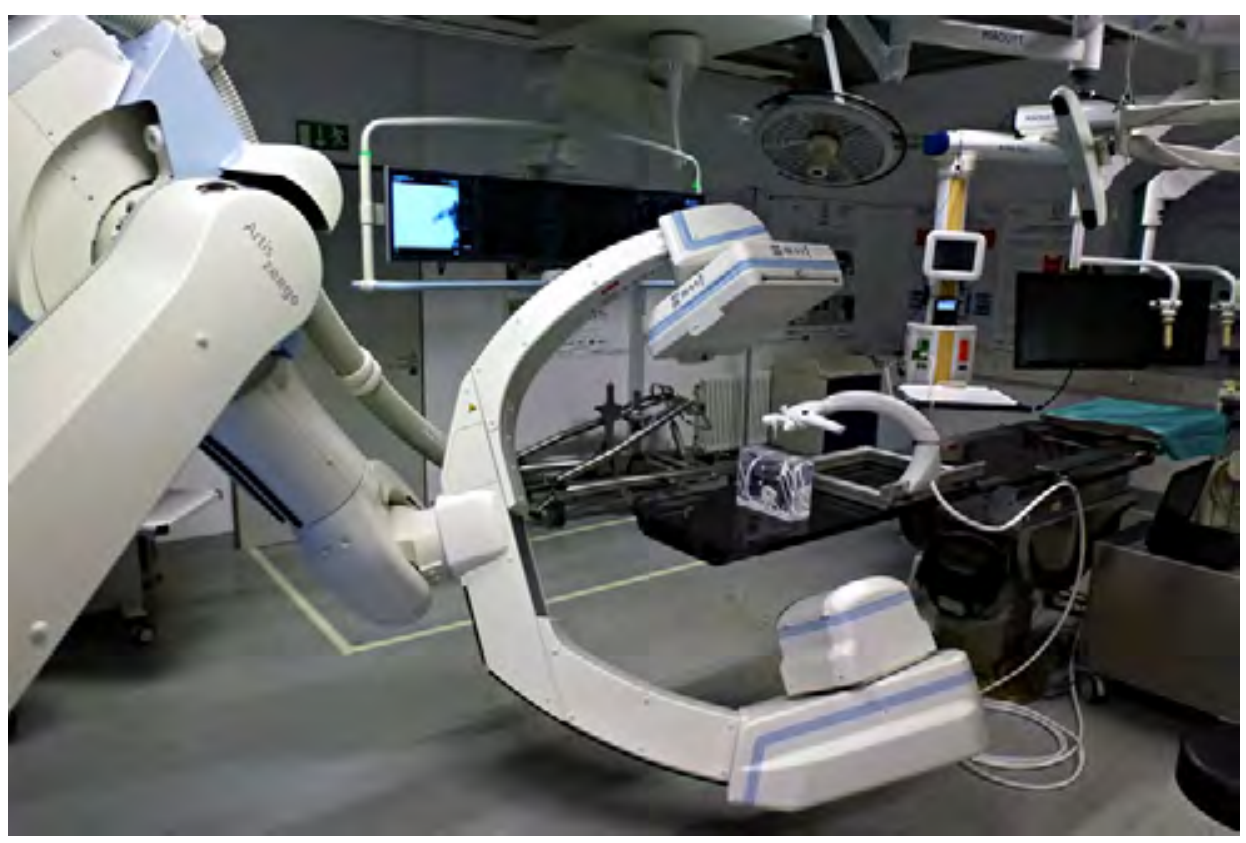

Figure 7.3: System integration with $\mathrm{CBCT}$ at the facilities of Fraunhofer.

In addition, the system was integrated with a Siemens [35] Artis zeego cone beam CT system (Fig. 7.3) at the facilities of the Fraunhofer Project group for Automation in Medicine and Biotechnology (PAMB) [224]. Needle placement at targets in a glycerine phantom of the thorax was successfully demonstrated.

\subsubsection{System redesign for MRI-guidance}

Redesign of the system for MRI-guidance was taken into account at the start of its development. It imposes requirements on the system's MR safety and MR compatibility $[225,226]$. MR safety considers the occurrence of magnetically induced forces and torques, radio frequency $(\mathrm{RF})$ induced heating and induction of voltages and their effect on a device. It distinguishes between 'MR unsafe', 'MR conditional' and 'MR safe' devices. MR compatibility refers to the effect of the presence of an MR (conditionally) safe device on the quality of the acquired diagnostic (image) data. Whereas the X-rays in CT are limited to a single plane in the centre of the CT scanner bore, the magnetic field and RF signals used in MRI encompass a volumetric range in and around the MRI scanner bore, augmenting the range in which material constraints are applicable to the system.

Candidate construction materials for MR safe and MR compatible design correspond to large extend to X-ray transparent design: rubbers, plastics, woods, 


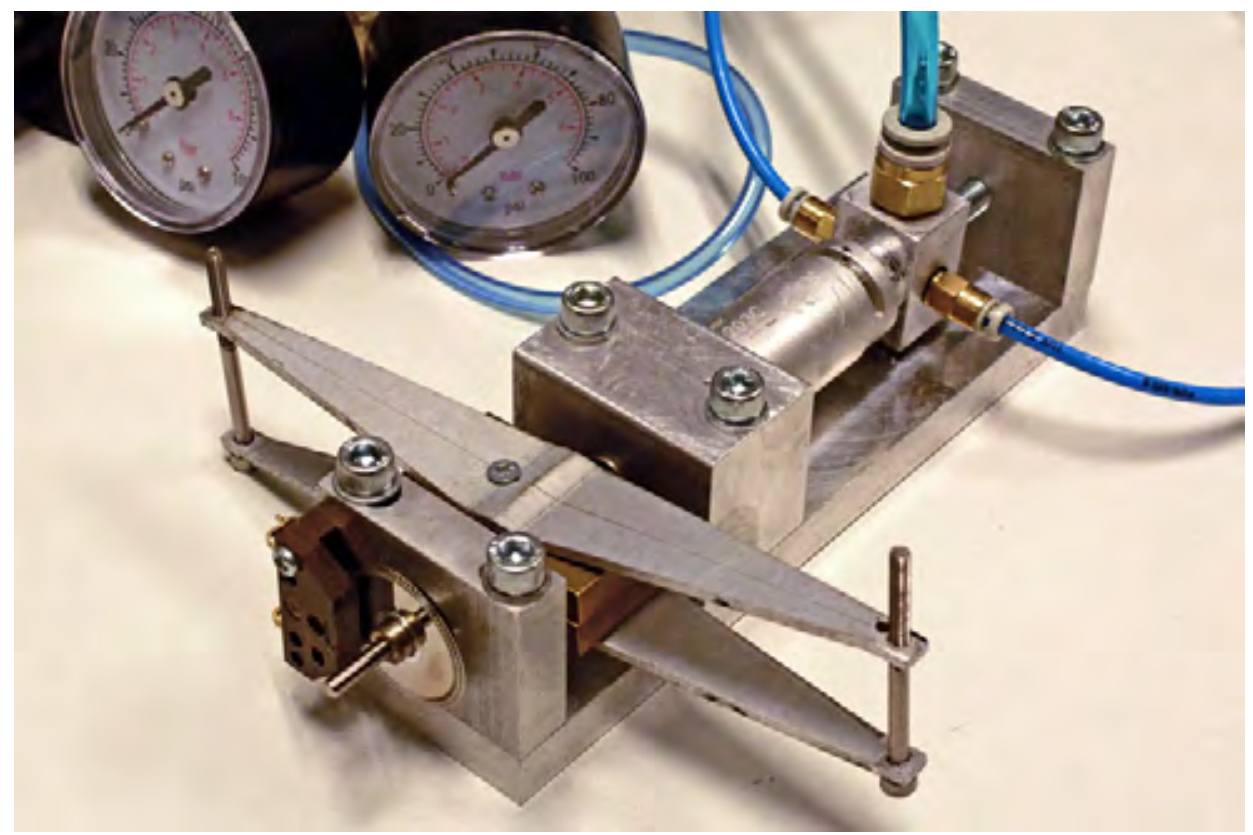

Figure 7.4: Experiment setup to investigate position control of a vane motor.

(non-conductive) fibre reinforced epoxies and ceramics. In addition, a selection of non-ferromagnetic metals and alloys may be used in MR conditional systems, such as aluminium, titanium, brass, bronze and stainless steel.

The size and shape of the system can be maintained, being designed to fit the typical $\varnothing 60 \mathrm{~cm}$ bore of MRI scanners. The current materials of the OM and upper half of the LM arm are both X-ray transparent as well as MR safe. The aluminium oxide of the fiducial markers should be replaced by water or gadolinium contrast agent filled spheres to be visible on the MR images. The fibre optic encoders measuring the worm angles are MR safe, provided that the fibres are elongated for a more remote location of the connecting electronics. The carbon nanotube electrical circuits of the locking system could be replaced by fibre-optical switches. The LM foot contains several structural parts of steel which should be replaced by non-ferromagnetic metals or alloys for an MR conditional system. Alternatively, the LM could be redesigned in plastic, glass fibre reinforced epoxy and ceramics for an MR safe system. Implementation of the complete pneumatichydraulic locking system in rubber, plastic, and glass fibre reinforced epoxy is technically feasible, as demonstrated by the current design of ball joint 2 and its piston and cylinder. The electrical pneumatic valves should be moved to a more remote location, using tubes to cover the distance.

To replace the electrical servomotors, development has started to incorpor- 


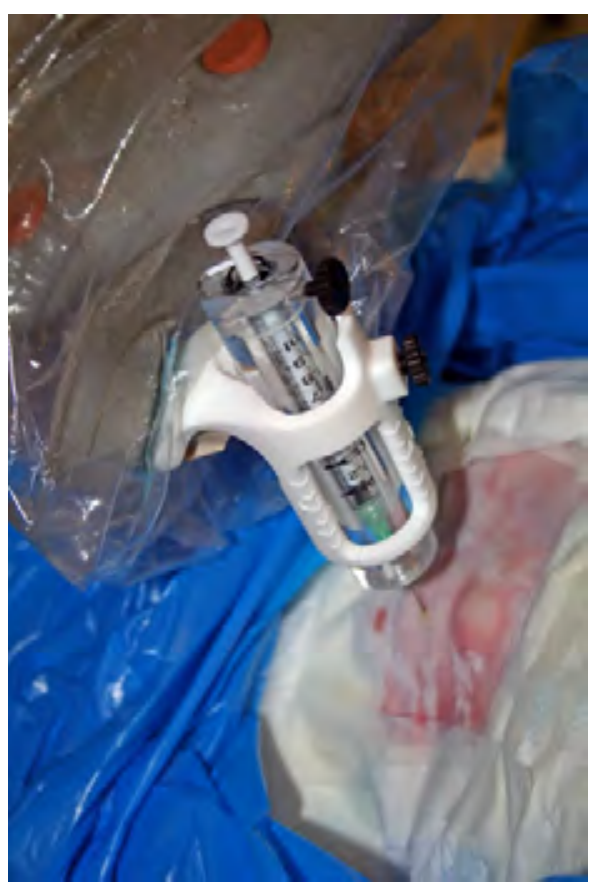

(a) Needle insertion after alignment of the syringe guide.

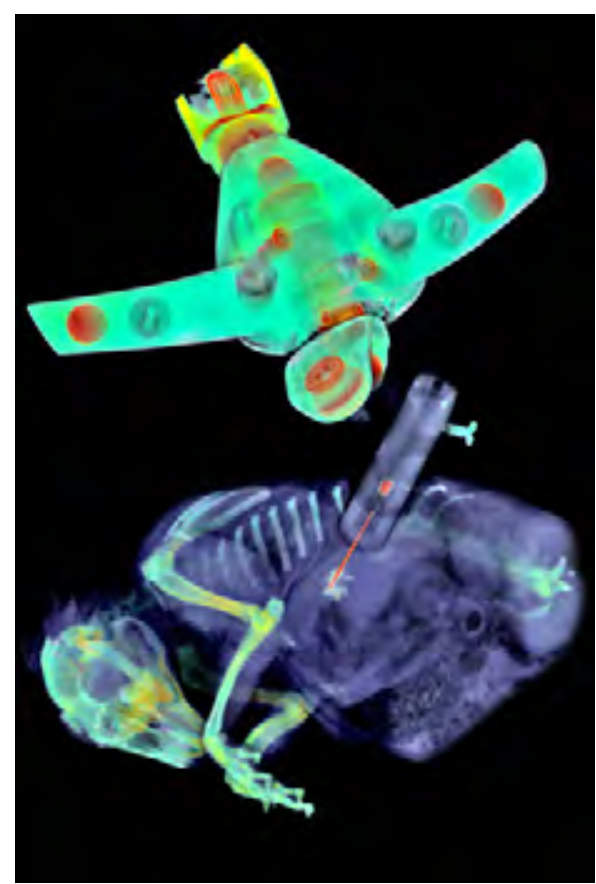

(b) 3D CT image of the system and rabbit after injection.

Figure 7.5: Deployment of the system for precision intercellular injection of holmium microspheres for radioembolization of a subcutaneous tumour in a rabbit at the facilities of the UMCU.

ate MR safe and compatible actuation by means of a pneumatic or hydraulic vane motor. Vane motors are simple, compact and easily miniaturized, have an excellent power-to-size ratio and can deliver both high torque and high rotational speed $[227,228]$. X-ray transparent and MR safe ceramic versions are available [229]. A challenge lies in their position control [230], and an experiment setup has been realized consisting of a vane motor, adjustable prony brake (to model drive system friction), incremental encoder (for position feedback) and adjustable rotational inertia in series, to investigate the suitability of different control strategies for different trajectories and load cases (Fig. 7.4). A high transmission ratio, MR safe brake systems and pneumatic valves close to the pneumatic vane motor to reduce the volume of compressible air in between are proposed to achieve the required positioning performance. The brakes and valves could in turn be remotely controlled via pneumatic, hydraulic or cable transmissions. 


\subsubsection{System application for radioembolization}

The needle placement system has been deployed in an animal study for precision intercellular injection of holmium microspheres for tumour radioembolization, in collaboration with the University Medical Center Utrecht (UMCU) [231] and Quirem Medical [232]. A novel add-on was created to guide and fixate a radiation shielded syringe. The needle placement system enabled successful coverage of a subcutaneous tumour in a rabbit by multiple adjoining injections of holmium microspheres (Fig. 7.5).

\subsubsection{System application for MWA of liver tumours}

The system readily received approval of the Medical Ethical Committee of the University Medical Center Groningen (UMCG) [233] and the Dutch Health Care Inspectorate (IGZ) [234] for deployment in clinical practise for MWA of liver tumours at the UMCG, emphasising the system's clinical acceptability. A randomized clinical trial has started (Fig. 7.6), which aims to clinically validate the system's guided method in comparison to the freehand method. Both methods will be employed for MWA needle placement in 21 tumours each. At the time of writing, 6 lesion in 5 patients have been targeted using the system's guided method and reached successfully.

\subsubsection{DEMCON Medical Robotics}

The success of the needle placement system as output of this research has inspired the launch of a new branch of the DEMCON holding: DEMCON Medical Robotics [235]. Within the field of medical robotics, its focus will be to develop and commercialize application driven technology for more effective and efficient diagnostics and intervention. Its first goal is to revise the design of the needle placement system for production and bring it to market. 


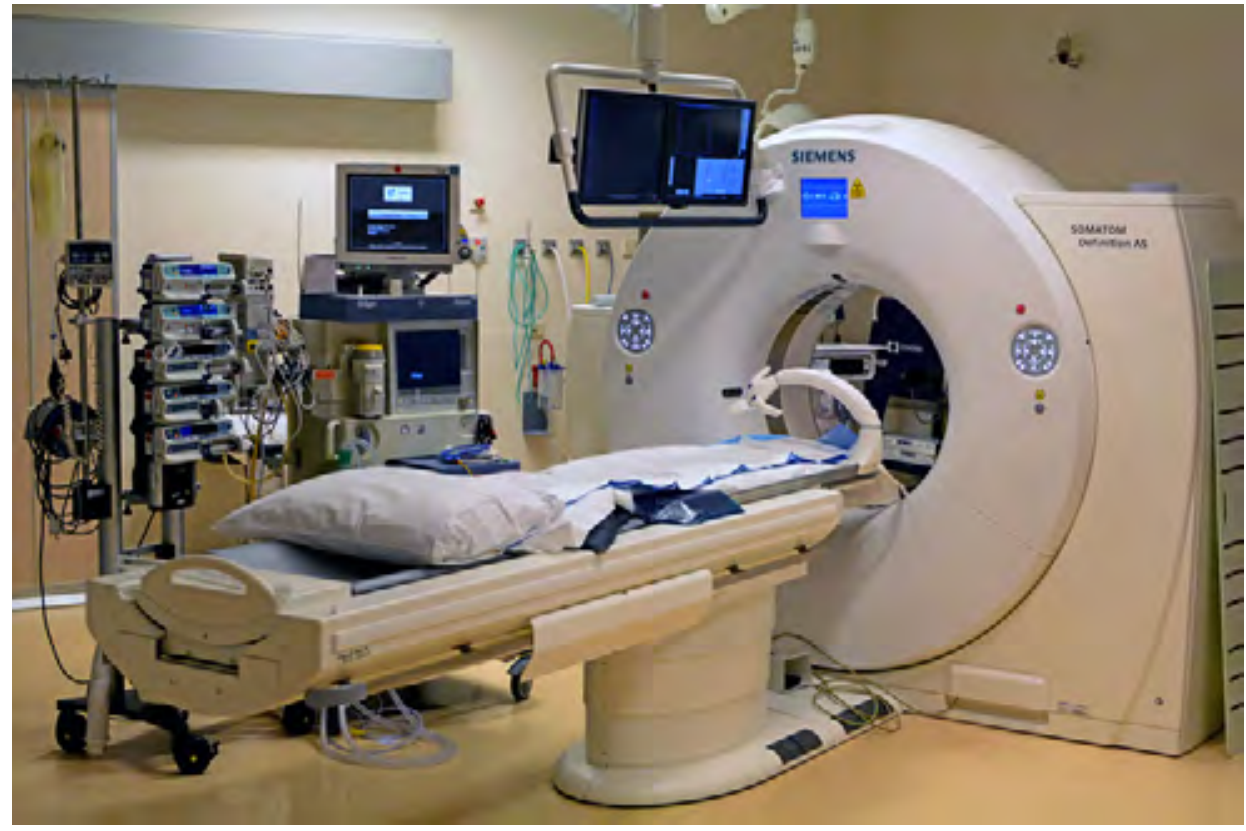

(a) System integration in the intervention suite prior to patient accommodation.

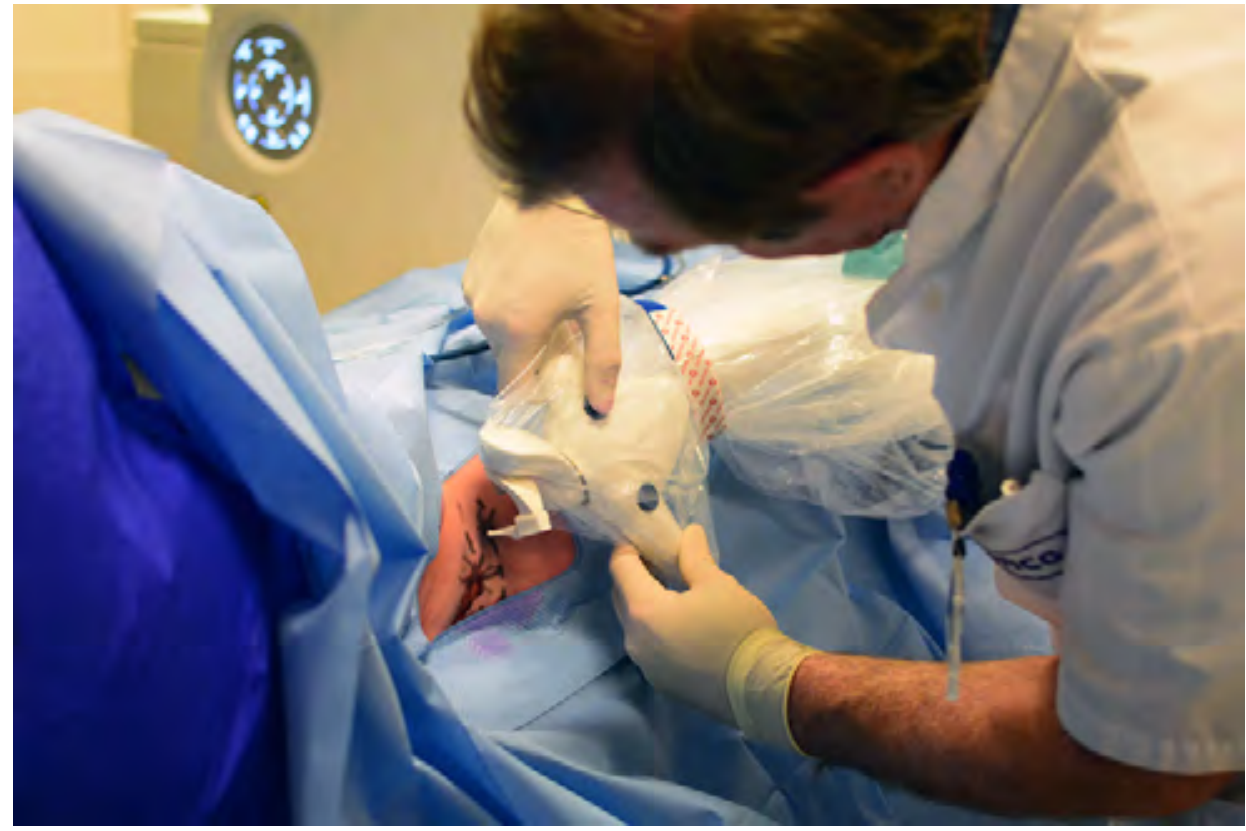

(b) Manual placement of the OM to coindide the RCM with the retrieved skin entry point.

Figure 7.6: Clinical deployment of the system at the UMCG. 


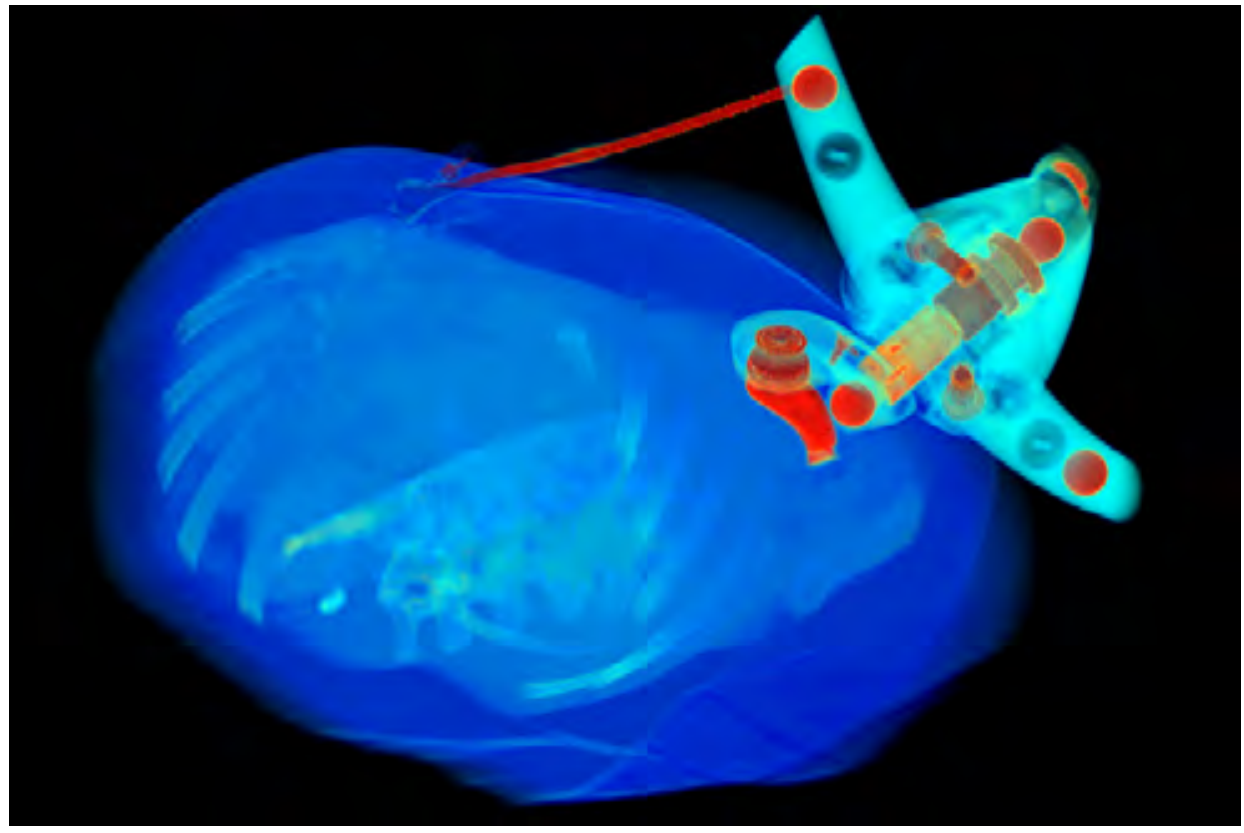

(c) 3D CT image for OM-to-CT registration and specification of the target coordinates.

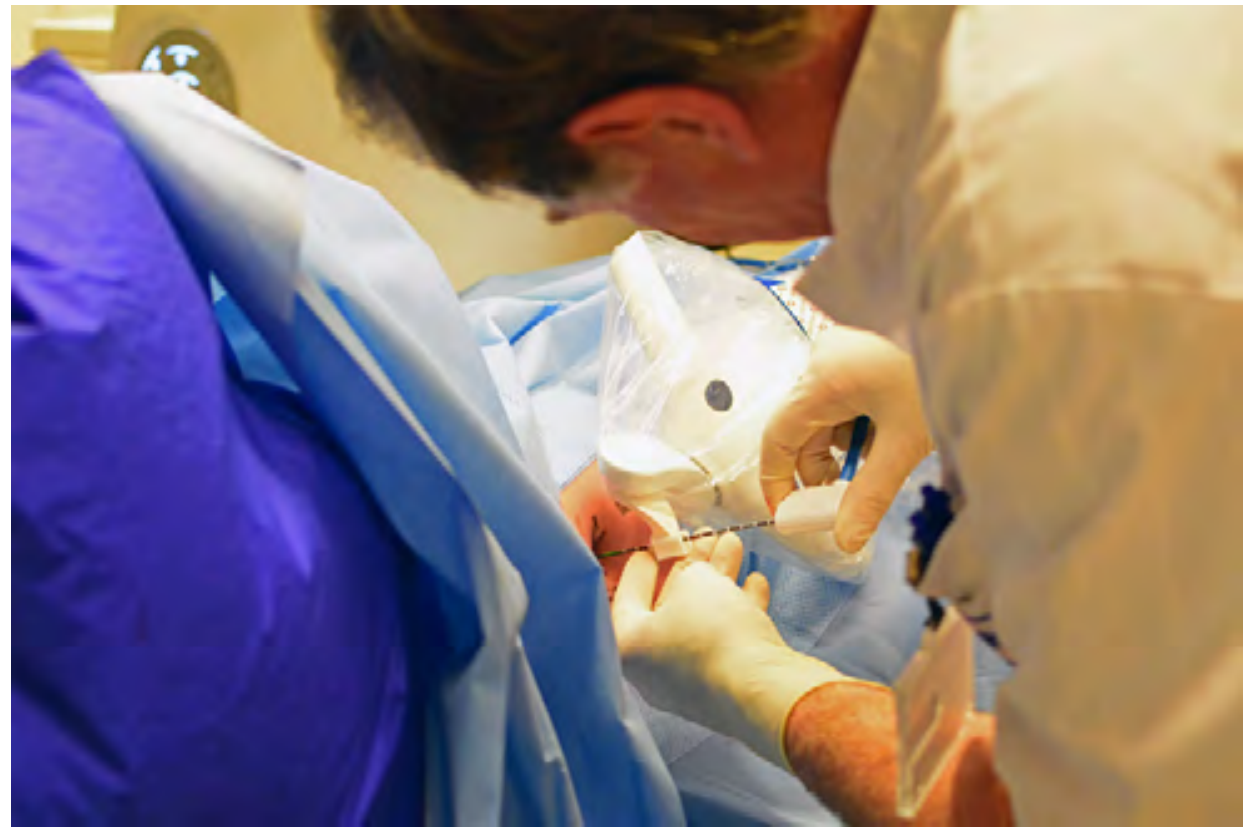

(d) Manual needle insertion after automatic aiming of the needle guide at the specified target.

Figure 7.6: Clinical deployment of the system at the UMCG - continued. 


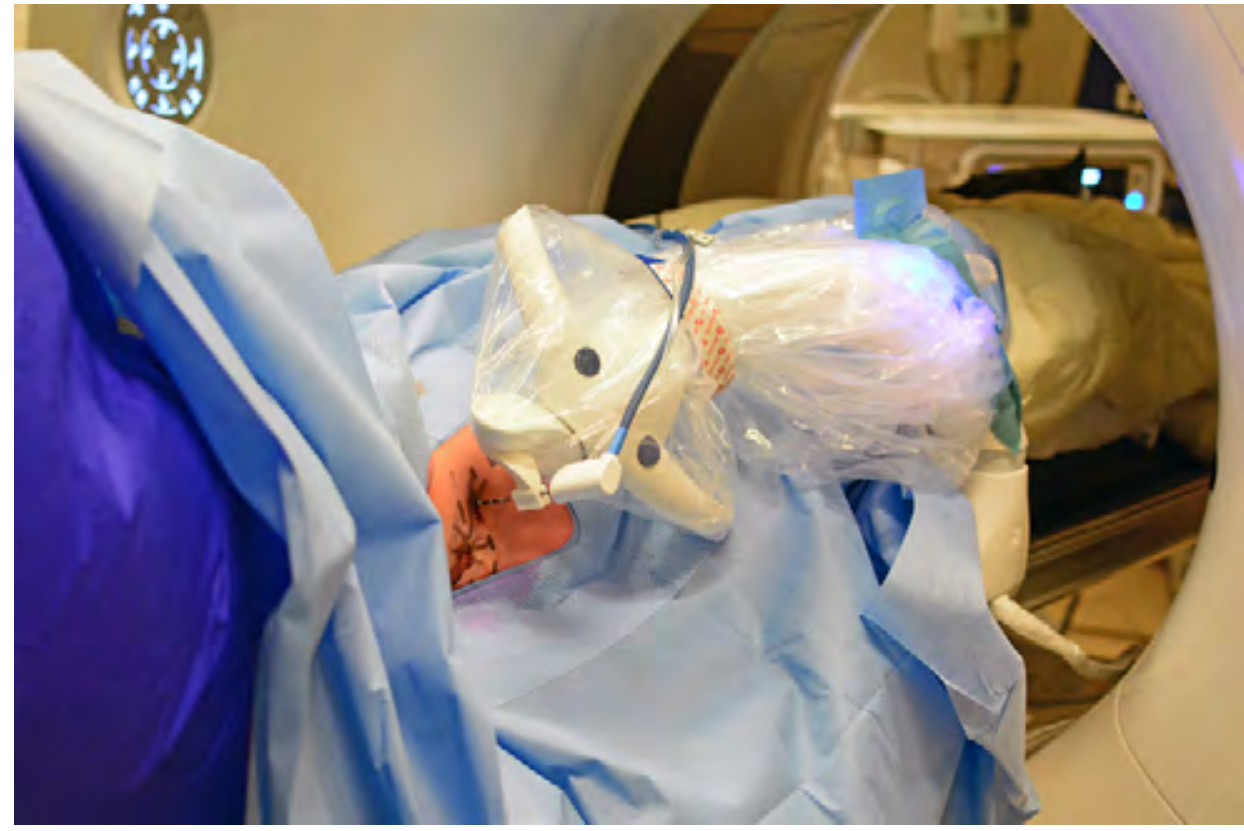

(e) Needle inserted under guidance of the system.

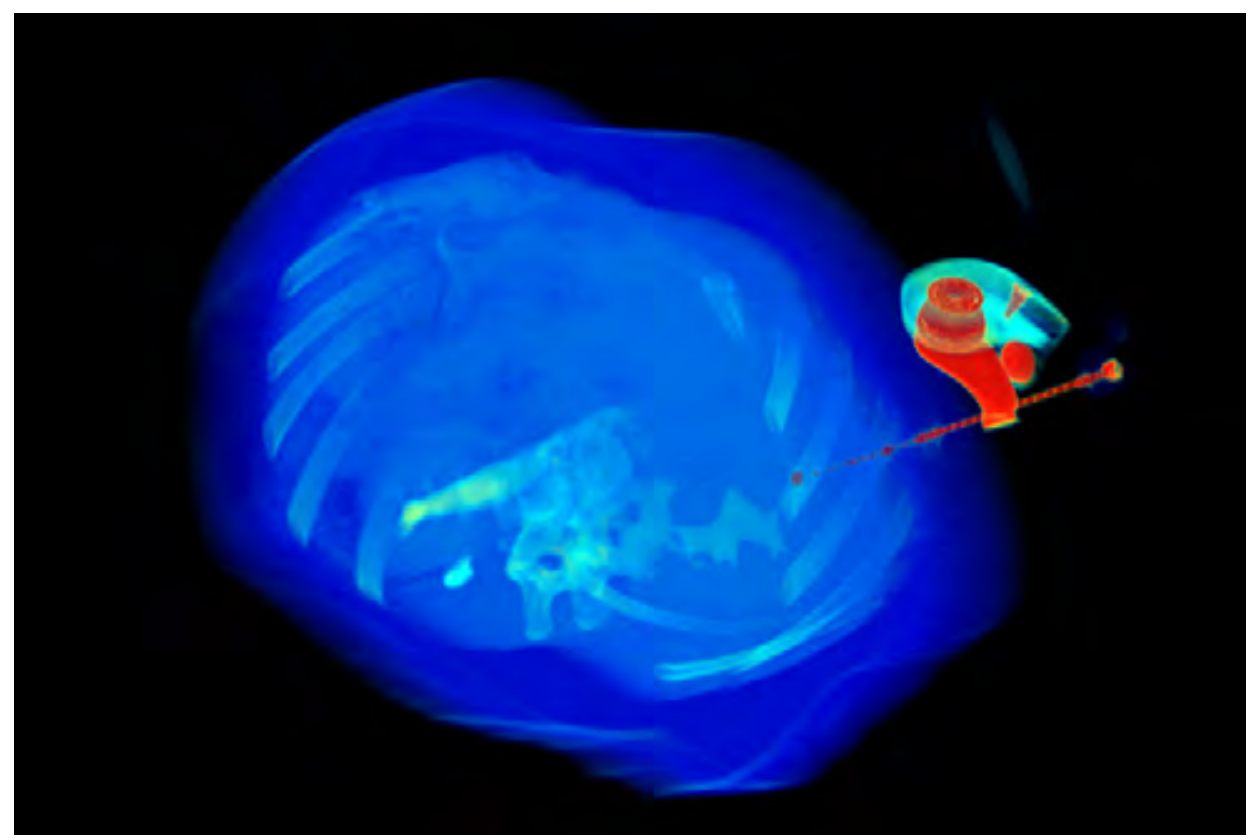

(f) 3D CT image after insertion for placement verification.

Figure 7.6: Clinical deployment of the system at the UMCG - continued. 


\section{Appendix A}

\section{Development history}

The strategic, architectural and detail design of the needle placement system and the considerations on its clinical acceptability, applicability and usability have been thoroughly discussed in the main chapters of this dissertation, as well as its verification and pre-clinical validation. The path from scratch to a system ready for clinical deployment has remained underexposed. The technical challenges, effort and perseverance behind the deceivingly sleek design and simple usability of the system are easily overlooked by the uninitiated, and its functionality, performance and smooth clinical integration taken for granted. An illustrated high-level overview of the system's development history is presented to provide some perspective on its expanse.

The research started early 2011 with a scope on minimally invasive imageguided interventions. Various percutaneous procedures and focal therapies were reviewed, including biopsy, radio frequency ablation, microwave ablation, ethanol ablation, cryoablation and brachytherapy (Fig. A.1). Simultaneously, the capabilities and characteristics of different imaging technologies were analysed, including (tracked) 2D and 3D ultrasonography (US), computed tomography (CT) and magnetic resonance imaging (MRI), and the option of rigid and deformable image fusion. The opportunities for mechatronic system development were explored (Fig. A.2). We attended various percutaneous procedures at multiple medical centres to analyse current clinical workflows, including MRI-guided prostate biopsy, US-guided prostate brachytherapy, and CT-guided liver tumour ablation (Fig. A.3) and lung biopsy. We decided to focus on the development of a system to improve CT- and MRI-guided needle placement in the thorax and abdomen. A review of the state of the art indicated a wide variety of systems with overall poor clinical adoption (chapter 2). In the author's personal opinion, the pitfalls come down to:

- providing no or insufficient improvement to establish user-independent and adequate performance,

- solving one problem for another, by burdening the user with multiple substitutive cumbersome adjustments or mental calculations,

- offering insufficient clinical applicability due to a limited action range or large size,

- automating for the sake of automation, often at the cost of complexity, size 


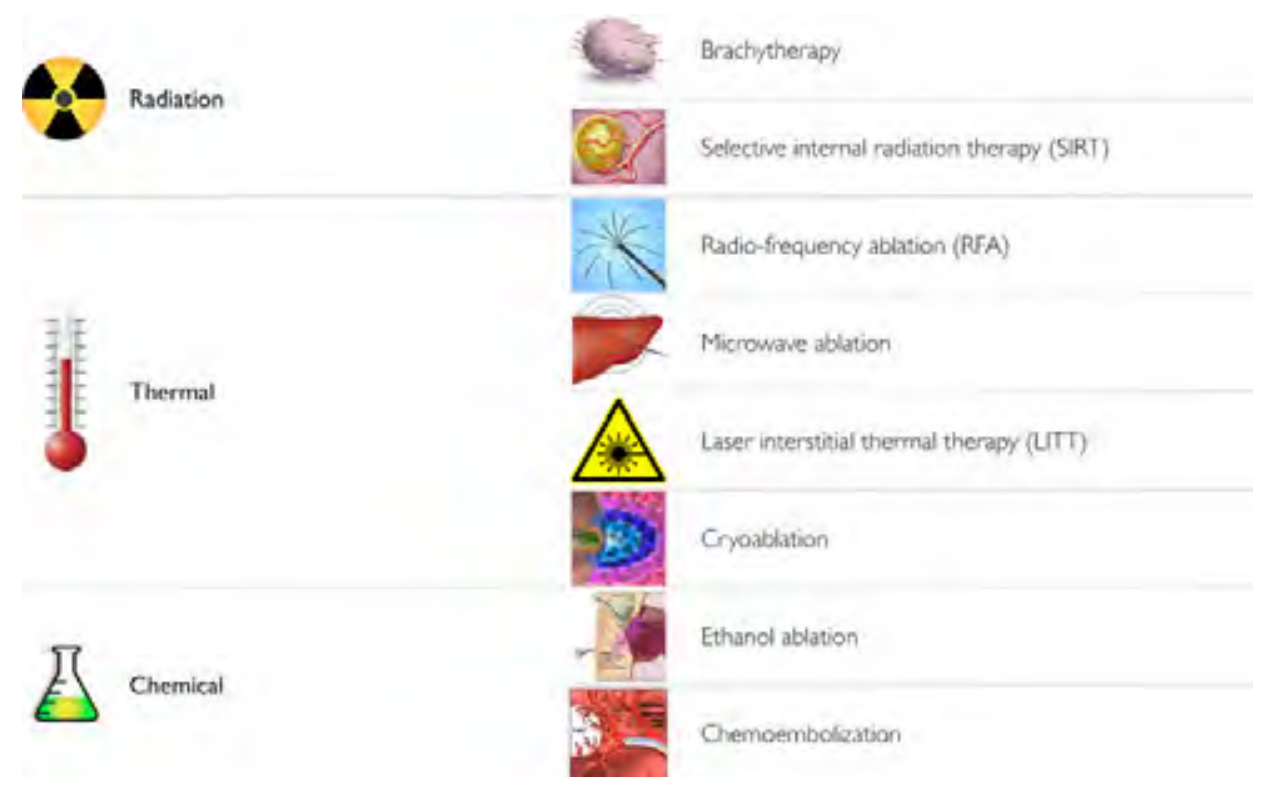

Figure A.1: Overview of focal therapies in oncology (2011).

and usability, and putting clinical acceptability in jeopardy especially when automating insertion, and

- implementing large, off-the-shelve robotics to create a generic, do-it-all platform without a dedicated plan for clinical integration.

This shaped our objective and design philosophy to develop a clinically acceptable, widely applicable and usable system that enables user-independent, precise needle placement in a single insertion, using lower radiation exposure and shorter procedure time, by wisely choosing functionality, thought-through user interfacing and integration in and improvement of the existing clinical workflow, while introducing minimal substitutive user effort in return (chapter 3). A problem analysis of the conventional freehand method was performed and user requirements were extracted. The strategy of the system, its architecture and corresponding functions and requirements were elaborated. 


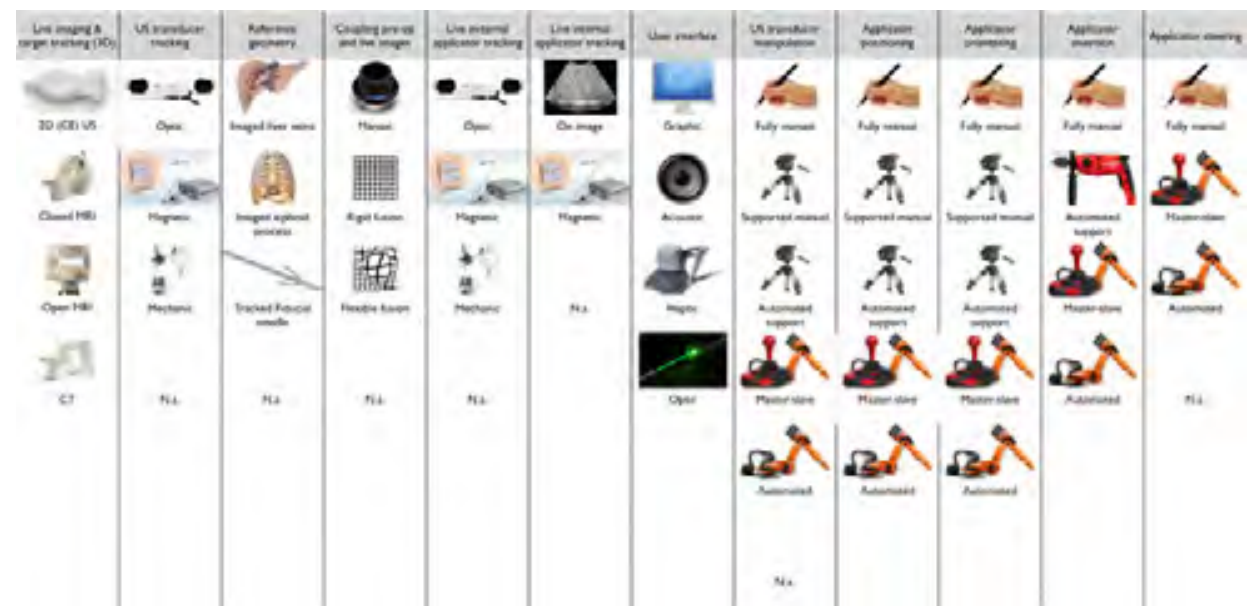

Figure A.2: Morphological chart of development opportunities (2011).

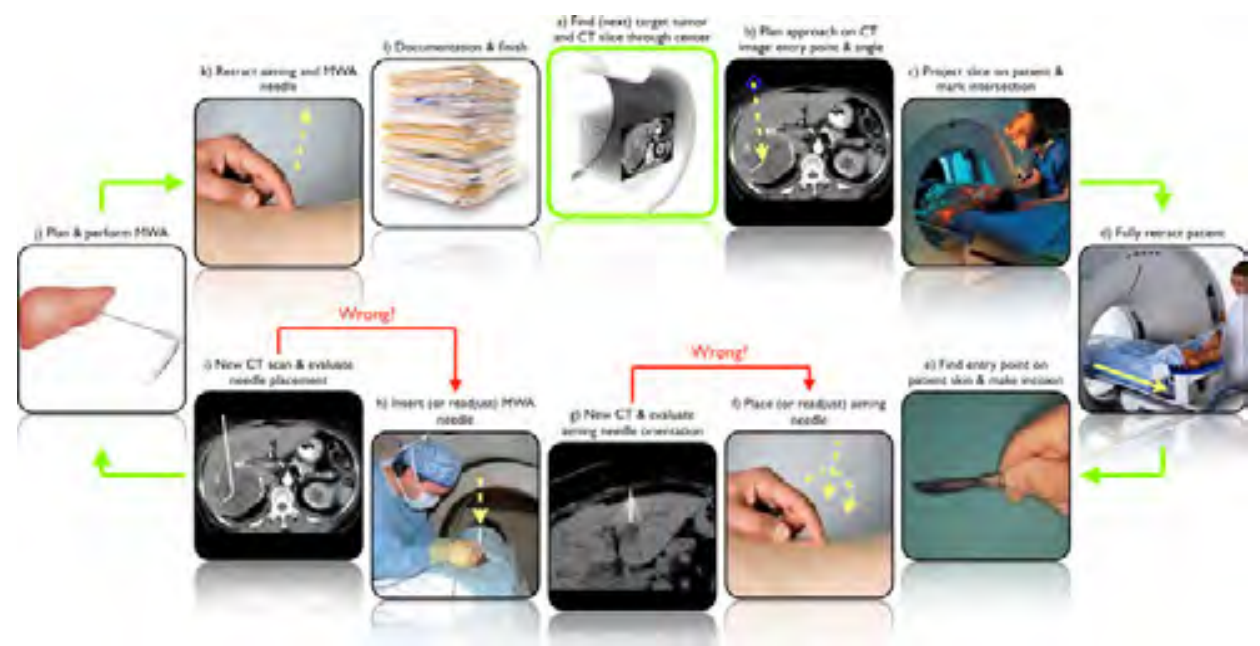

Figure A.3: Flowchart of CT-guided percutaneous liver tumour ablation (2011). 


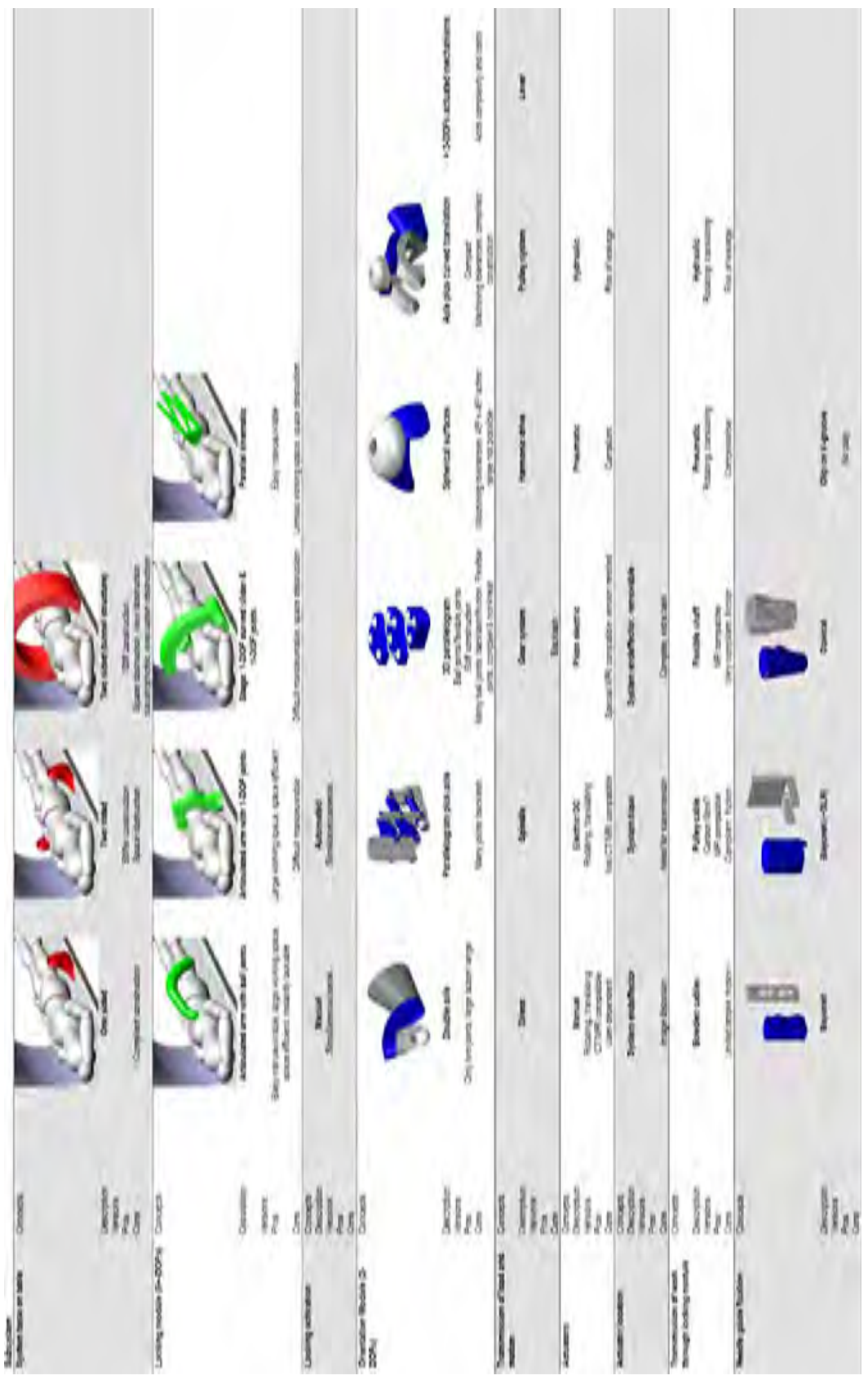

Figure A.4: Morphological chart of the different subsystems (2011). 

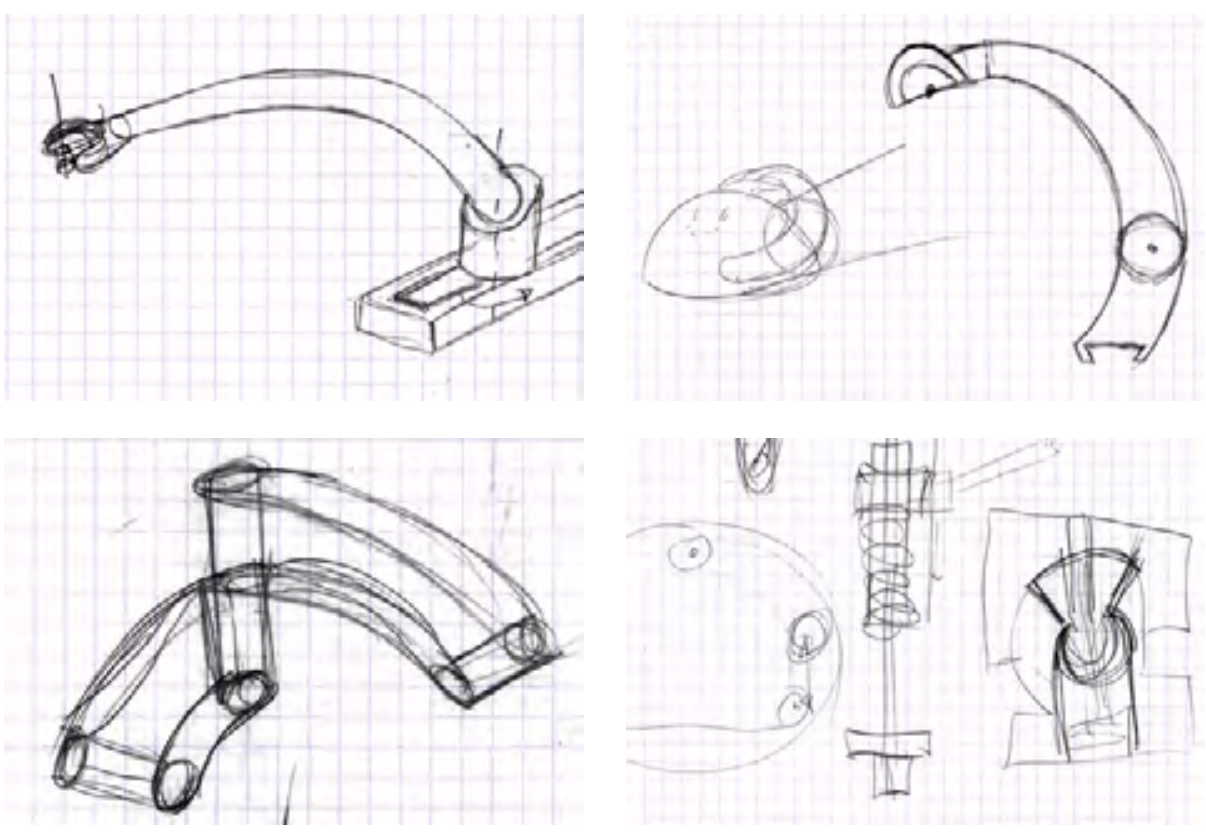

Figure A.5: Early sketches of the system (2011).

Various concepts for the different subsystems were considered and listed in a morphological chart (Fig. A.4). Figure A.5 shows some of the first sketches in which the outlines of the final system can clearly be recognized. By the end of 2011, the design had converged and was captured in a first CAD model of the system (Fig. A.6). The system's guided needle placement method and integration in the clinical workflow were thoroughly reviewed by multiple clinical users. To further substantiate the feasibility of the system's strategy, needle deflection was quantified in ex vivo experiments for various clinically used needles (chapter 4).

This marked the start of the detail design of the system (2012-2014), in order to enable the intended functionality. Mockups of the locking module (LM) and orientation module $(\mathrm{OM})$ were created for workflow simulation and evaluation of the system's size, shape and action range (Fig. A.7). Objects of various materials and sizes were CT scanned to investigate their X-ray transparency and to demonstrate the feasibility of system-to-CT registration using fiducial markers (Fig. A.8). 


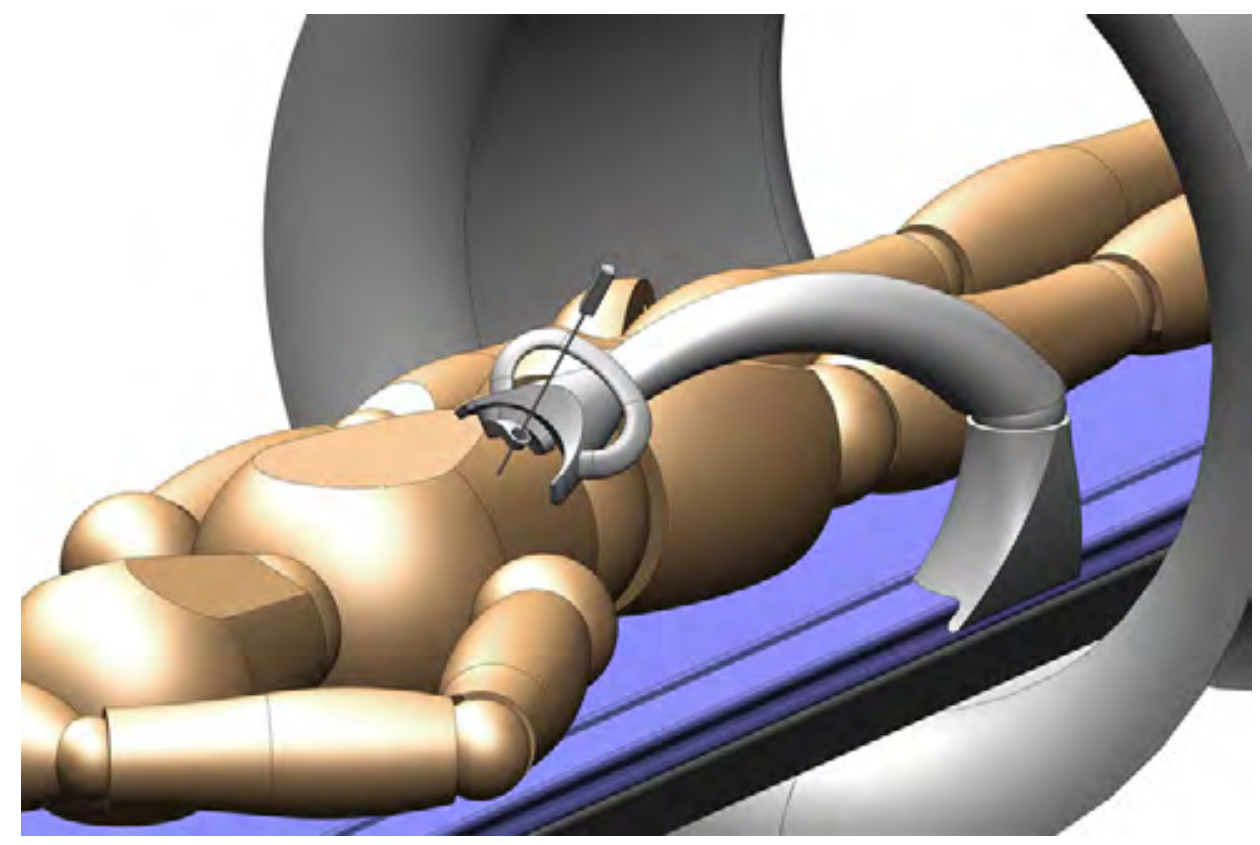

Figure A.6: First CAD model of the system (2011).

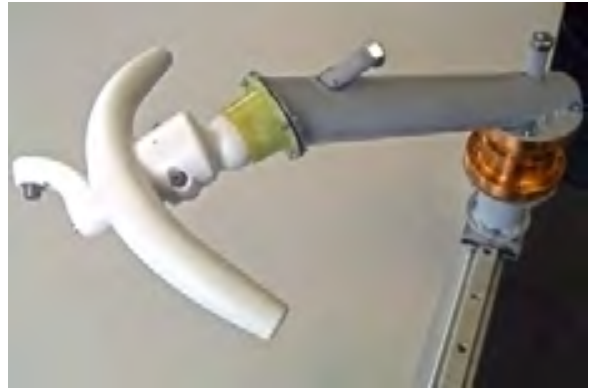

(a) Mock-up of the LM and OM for workflow simulation (2012).

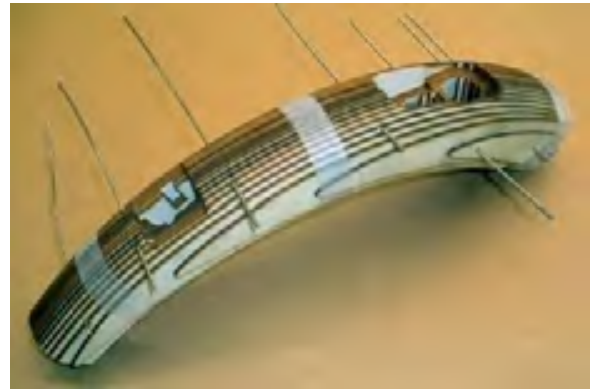

(b) Plywood mock up of the LM arm for shape evaluation (2013).

Figure A.7: Mock-ups to evaluate the design. 


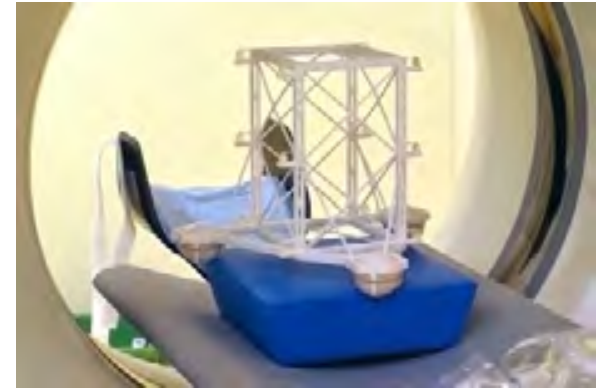

(a) SLS printed nylon frame holding $10 \mathrm{~mm}$ fiducial markers of various materials.

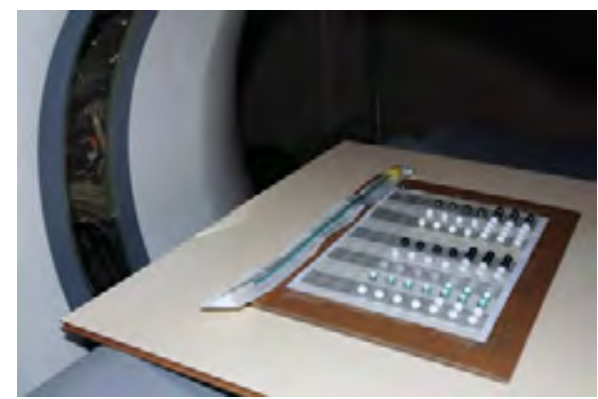

(c) Rows of $10 \mathrm{~mm}$ spheres of different plastics and ceramics.

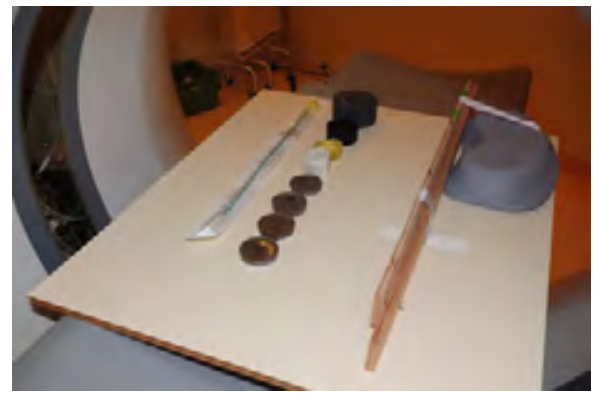

(b) A collection of custom material samples.

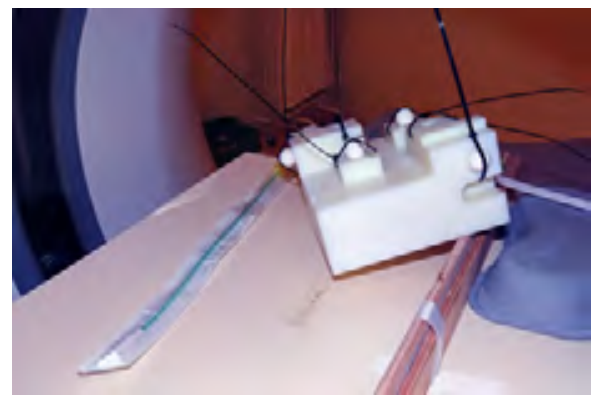

(d) Milled POM frame holding four $20 \mathrm{~mm}$ aluminium oxide fidicial markers.

Figure A.8: CT scanning to investigate $\mathrm{X}$-ray transparency and demonstrate the feasibility of system-to-CT registration using fiducial markers (2012).

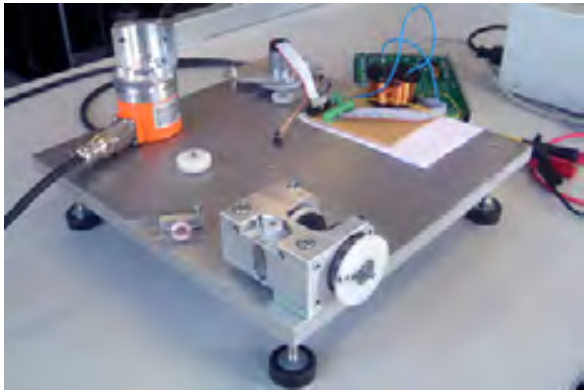

(a) Initial experiment setup to investigate the drive system (2012).

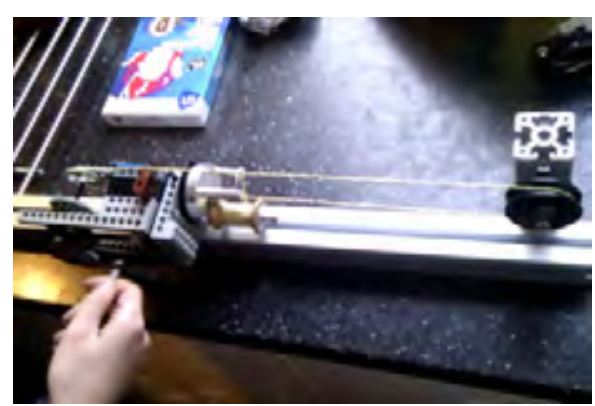

(b) Setup to spin an endless cable, build using LEGO (2012).

Figure A.9: Development of the drive system (2012-2014). 


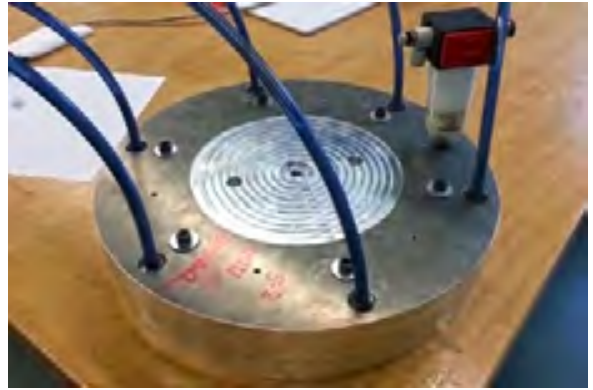

(c) Mould to cast a fibre reinforced endless cable (2013).

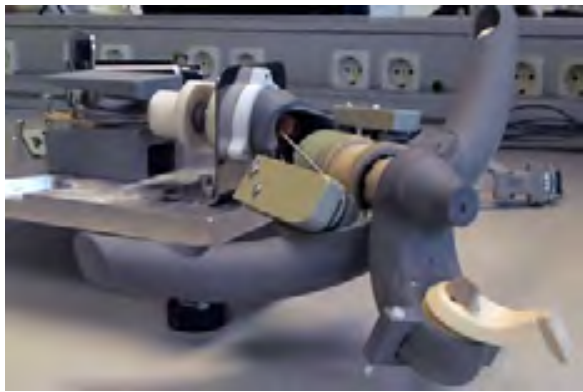

(d) Evolved experiment setup, incorporating the elaborated design of the OM (2014).

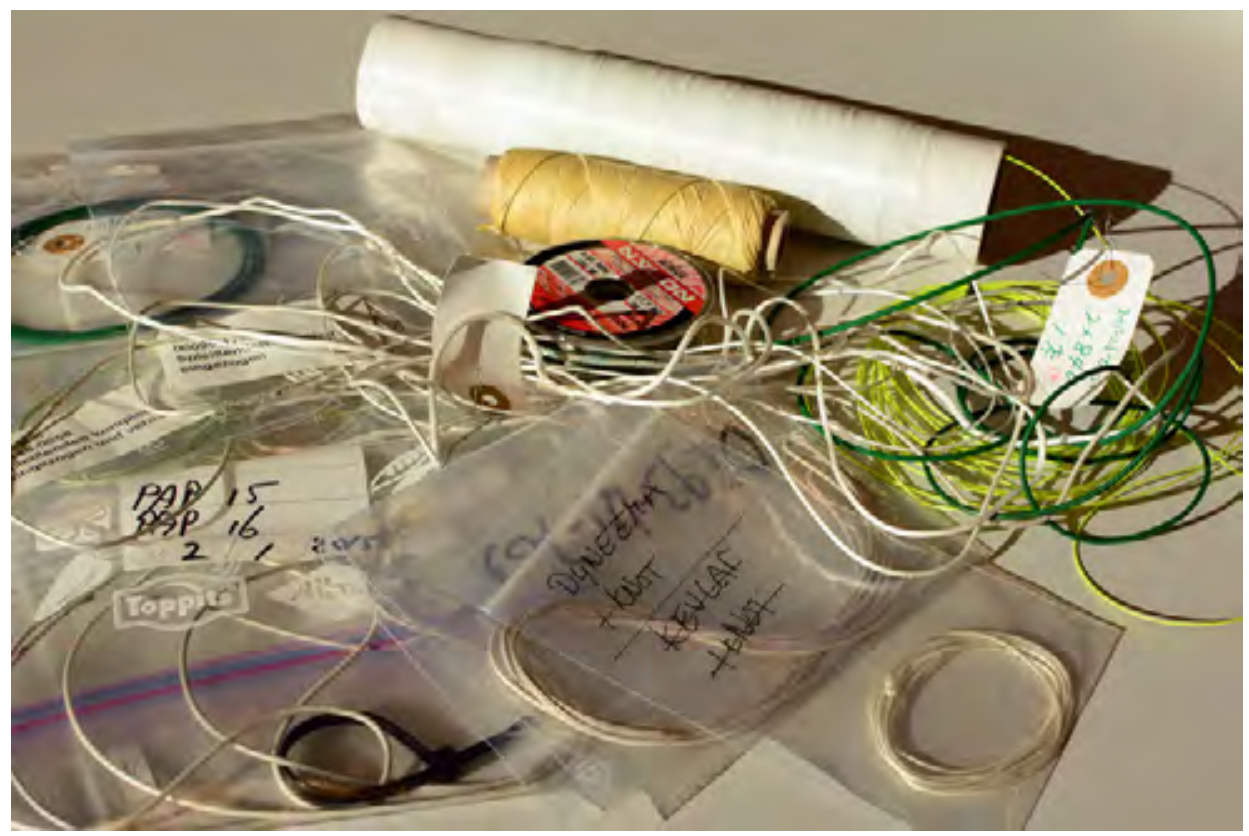

(e) Assortment of tested cables of various materials and constructions (2014).

Figure A.9: Development of the drive system (2012-2014) - continued. 

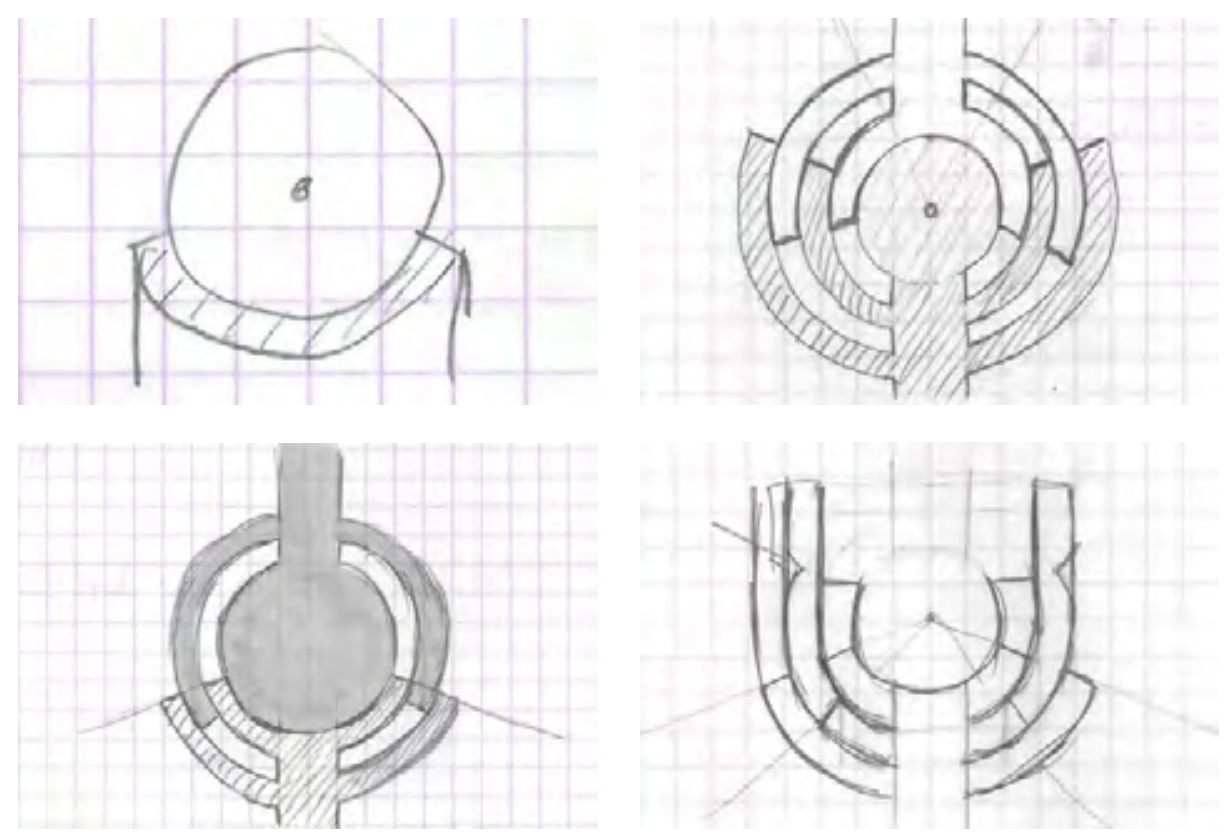

Figure A.10: Ball joint sketches (2012).

An experiment setup was build to investigate the drive system of the RCM mechanism, incorporating a servomotor, cable drive and worm gear transmission (Fig. A.9). We tried various cable materials including nylon fishing line, polyurethane rubber, PTFE, glass fibre, aramid and Dyneema, and tested their friction, slip, and life time performance. For long, creating an endless cable remained an unsolved issue, as we tried glueing, knotting, weaving and casting. A spliced Dyneema cable provided a breakthrough in 2013, which was iterated to perfection in close collaboration with Andre Bizot of Pro Air Paragliding. In continued testing we managed to quantify and compensate for the behaviour of the cables to shorten over time.

Figure A.10 shows sketches in search of a suited locking ball joint design. Fibre reinforced torsion springs to eliminate play in the drive system and composite ball joints were developed in collaboration with Futura Composites (Fig. A.11). The locking performance of various metal and composite ball joints with and without fibre reinforcement were investigated in an experiment setup (Fig. A.12). 


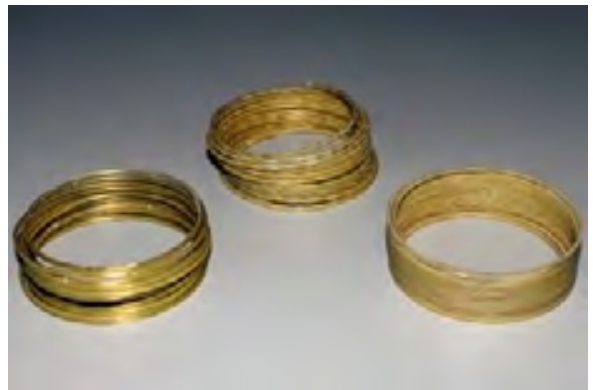

(a) Results of initial attempts to cast fibre reinforced torsion springs (2012).

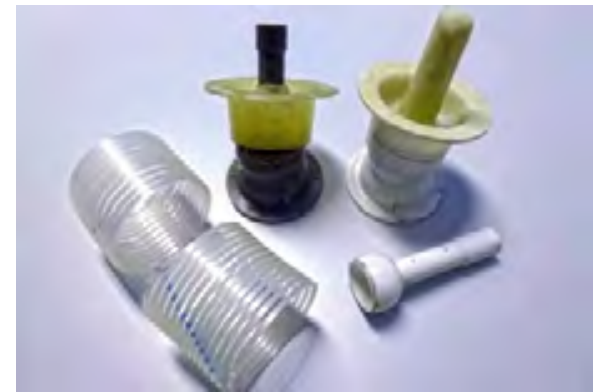

(b) Evolved torsion springs and composite ball joints (2013).

Figure A.11: Test samples of composite components, developed in collaboration with Futura Composites.

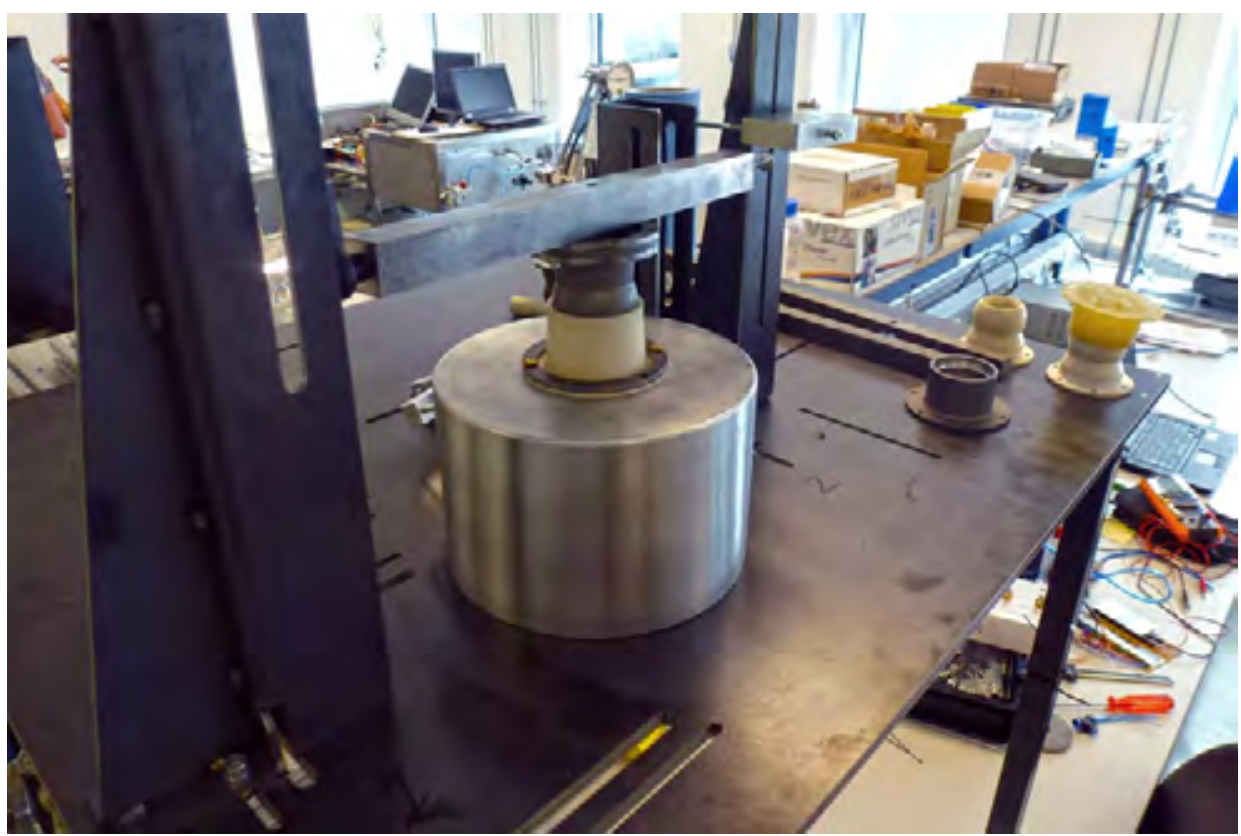

Figure A.12: Experiment setup to investigate the locking performance of various ball joint designs (2012-2014). 


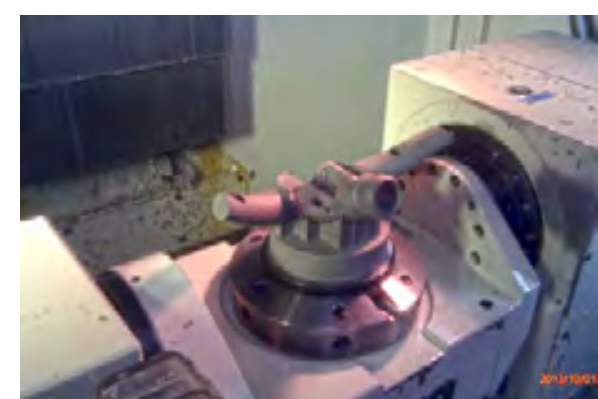

(a) Milling of the SLS printed OM main body at Parts \& Tools.

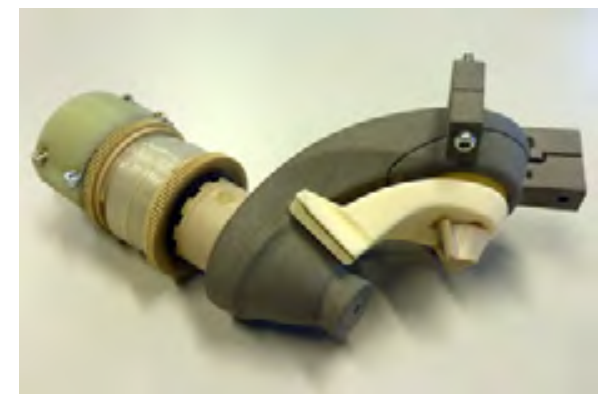

(c) Segment 1 and 2 assembly.

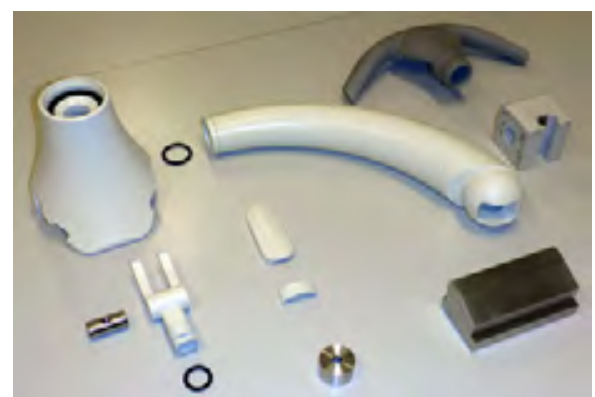

(b) Overview of various components before asembly.

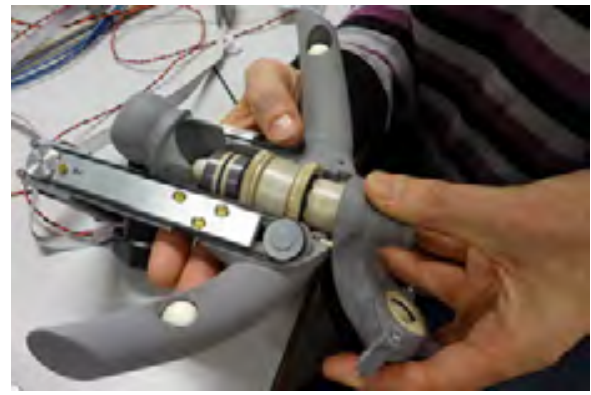

(d) OM subassembly with external motors.

Figure A.13: Realization of a prototype of the preliminary design (2013).

A prototype of the preliminary design was realized in 2013, which consisted of an empty mockup of the LM and a functional OM using externally attached electrical servomotors (Fig. A.13). The system was tested in combination with cone beam CT (CBCT) at the facilities of Philips Healthcare (Fig. A.14), and with CT at the facilities of the University Medical Center Groningen (UMCG) (Fig. A.15). 

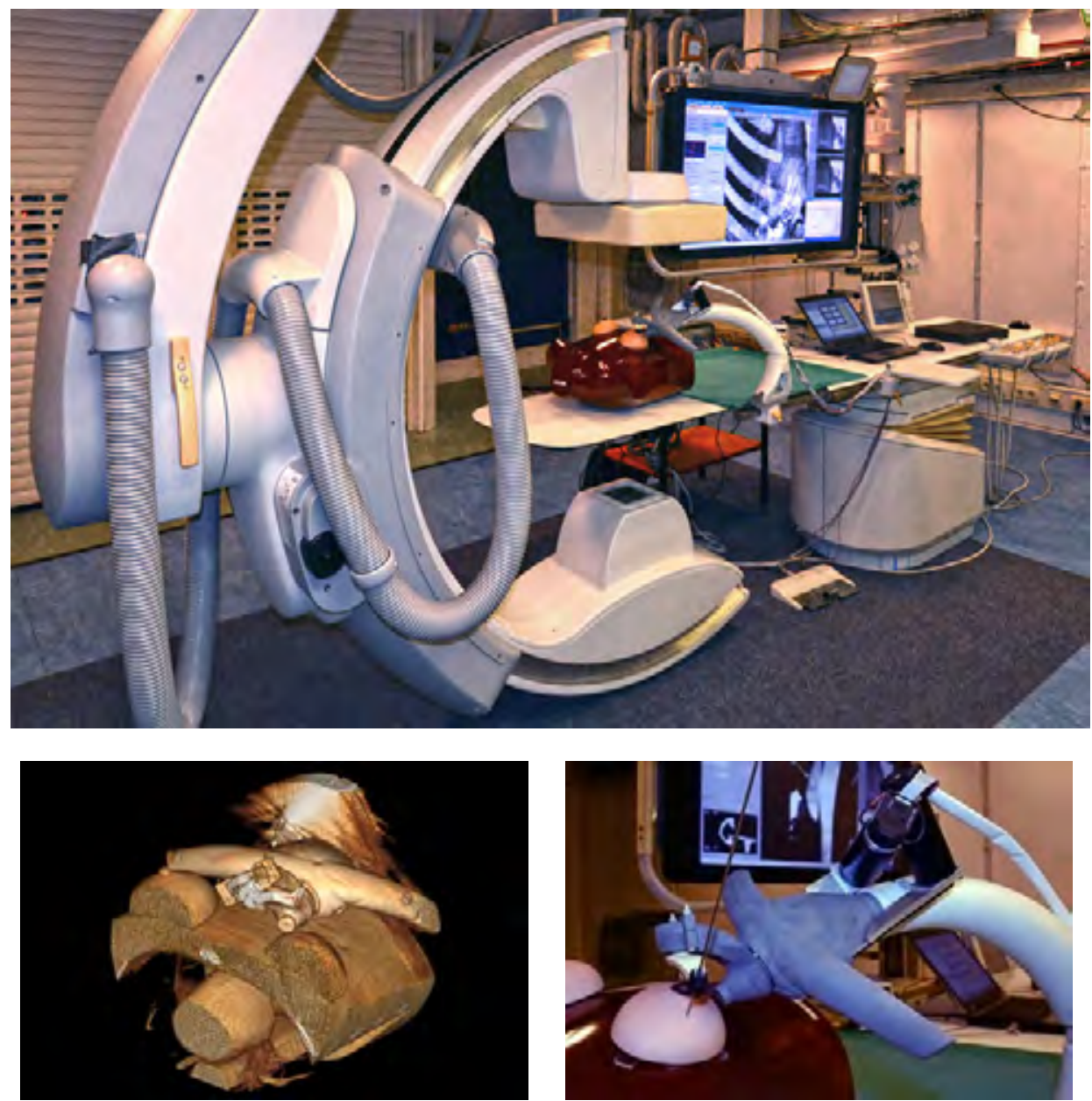

Figure A.14: Testing of a prototype of the preliminary design with CBCT at the facilities of Philips Healthcare (2013). 

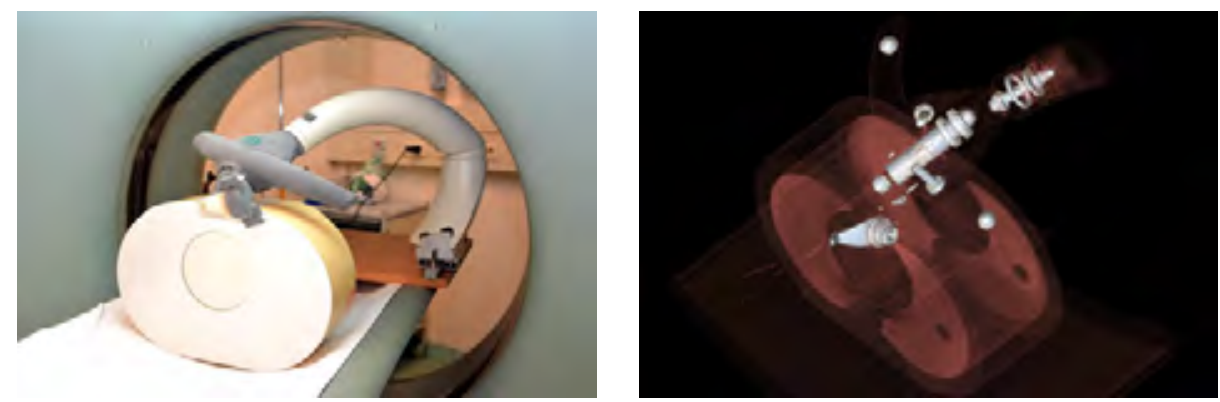

Figure A.15: Testing of a prototype of the preliminary with CT at the facilities of the UMCG (2014).
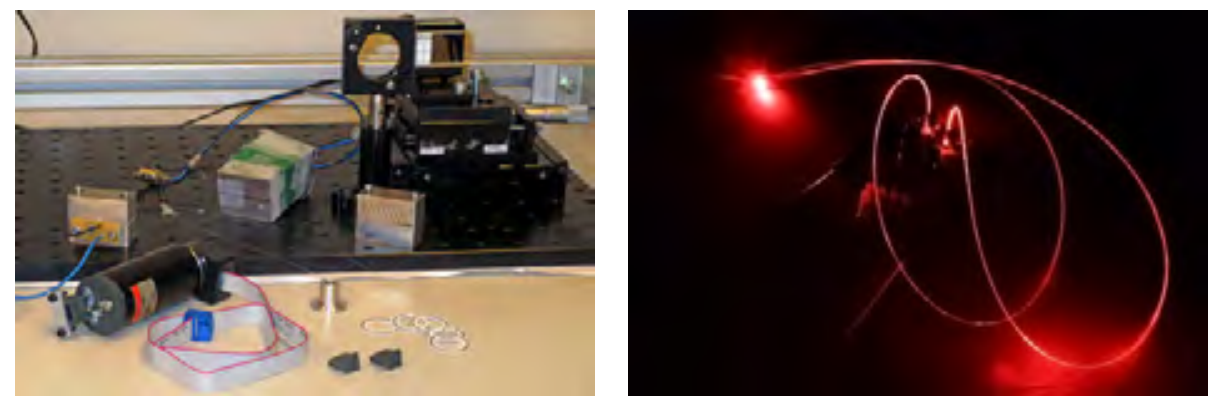

Figure A.16: Experiment setup to investigate the design of the fibre optic encoders (2013).

During performance testing of the endless Dyneema cable drive, it became clear that angular feedback would be unavoidable to account for variations in the transmission rate due to the radial stiffness of the rubberised pulleys, fluctuation in cable diameter and cable slip. This posed a new challenge: the development of custom fibre optic encoders (Fig. A.16). The optical path of light from one fibre reflected via a mirror to another fibre was investigated in an experiment setup and a proof-of-principle encoder was build and tested for reliability and maximum speed. For the final design, a custom printed circuit board containing light-emitting diodes and photodiodes for two encoders was developed and sized to fit inside the LM arm. 


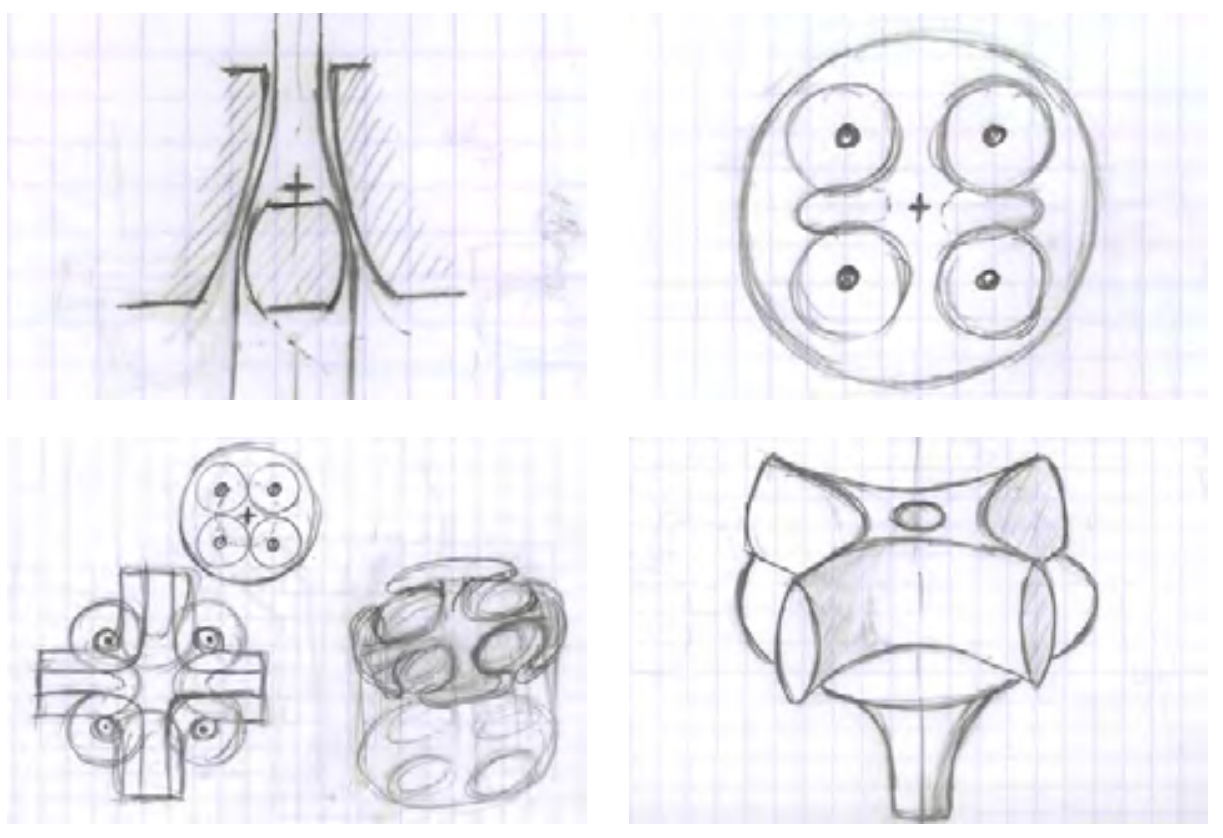

Figure A.17: Ball joint cable guide sketches (2013-2014).

Yet another challenge lay in the geometric design and material selection of a suitable guidance of the two endless cable drives through the 3-DOFs ball joint 2, which should function for any orientation, avoid wear of the cable and itself and induce minimal friction. In addition to the two cables passing twice, it should provide a safe passage for eight optical fibres and three carbon nanotube wires, and still allow for assembly. Figure A.17 shows several sketches drawn in search of a solution.

Various manual and automated mechanisms were conceptualized for the locking system. Manual locking was dismissed in consideration of usability. An automated pneumatic-hydraulic system was chosen for its relative simplicity and an experiment setup was build incorporating the pneumatic-to-hydraulic intensifier and three hydraulic cylinders to investigate its performance and technical design (Fig. A.18).

Push-buttons on the OM main body were selected as the most suited userinterfaces for control of the automated locking system, and different concepts were considered to create X-ray transparent signal routing, including fibre optic and aluminium electrical circuits. Carbon nanotube electrical circuits were chosen, and samples of this experimental material were supplied by Teijin Aramid. The conductive performance of the wires and the design of custom carbon electrical switches were investigated (Fig. A.19). 


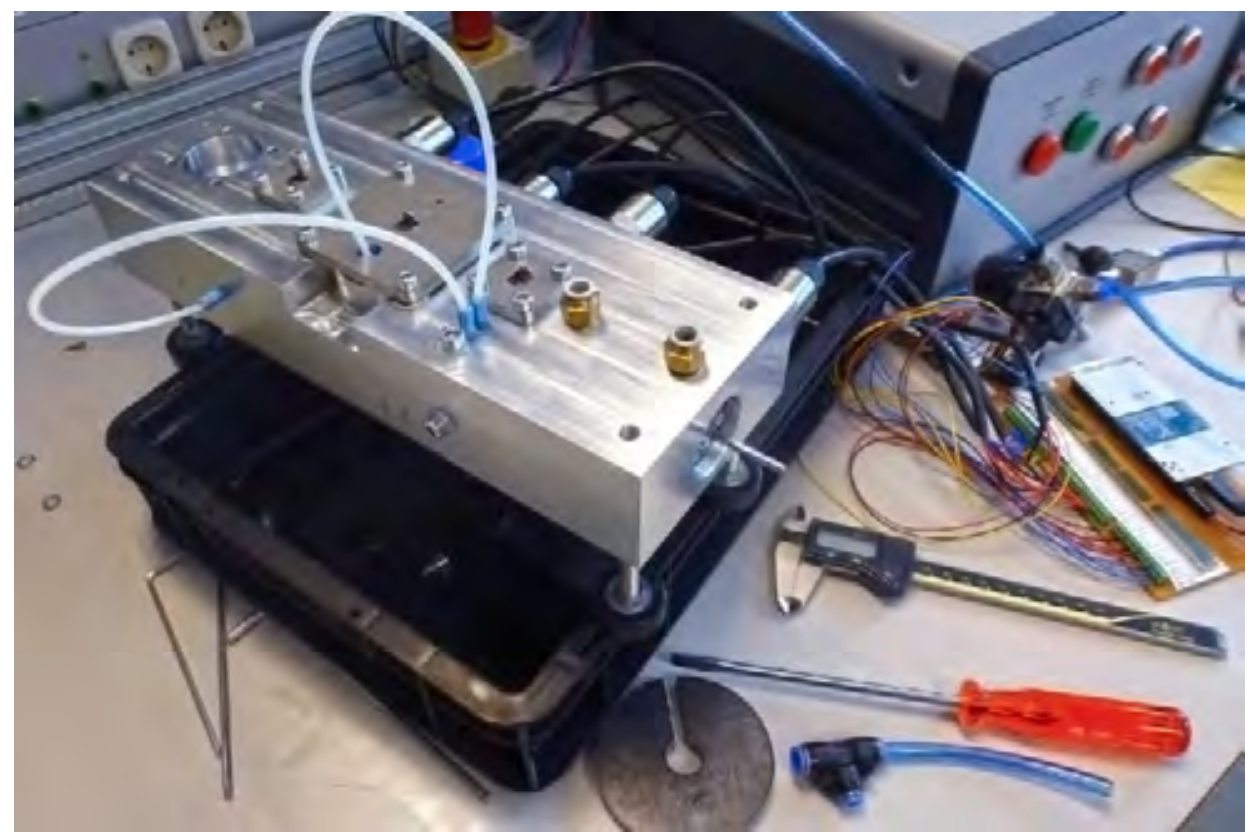

Figure A.18: Experiment setup to investigate the pneumatic-hydraulic locking system (2014).

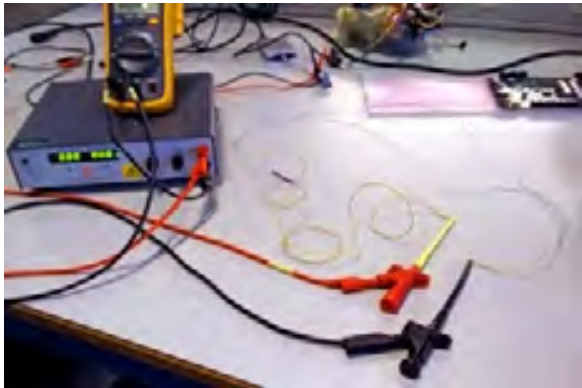

(a) Measuring the conductivity.

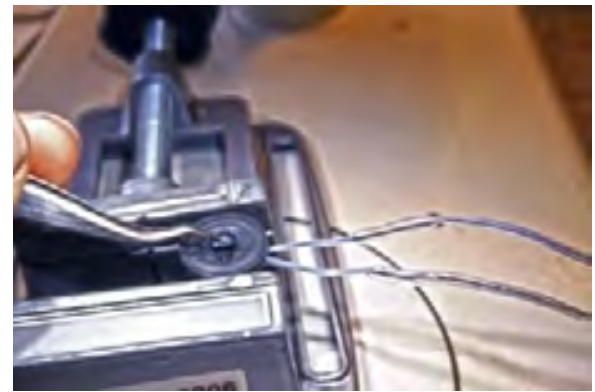

(b) Testing a custom carbon electrical switch.

Figure A.19: Experiments to investigate the use of carbon nanotubes as an X-ray transparent electrical circuit (2014). 


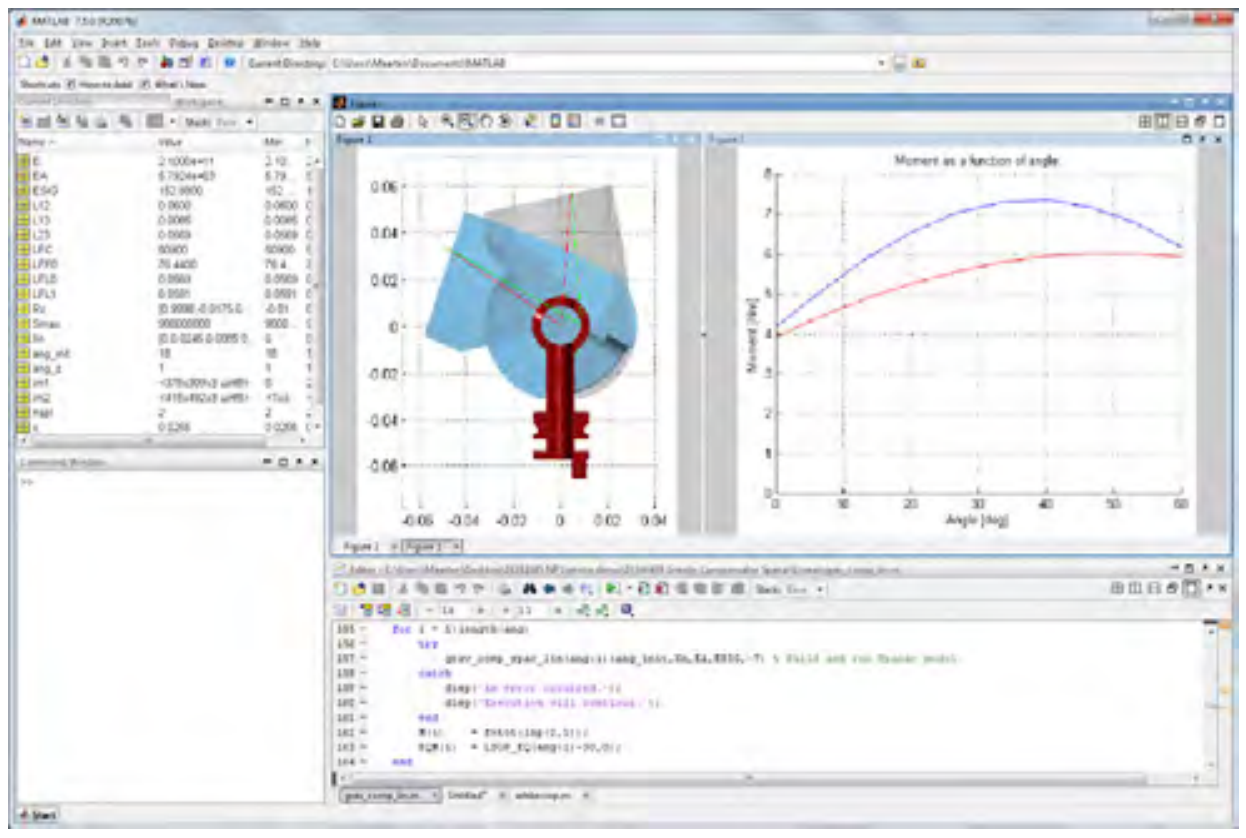

Figure A.20: Design of the gravity compensator using SPACAR (2014).

In order to maintain a static position of the LM arm when released without locking, a gravity compensator consisting of a preloaded eccentric spring mechanism was designed using flexible multibody modelling in SPACAR (Fig. A.20).

The static structural performances of segment 1 and the LM were analysed for various material combinations using a finite element method in Ansys (Fig. A.21).

Algorithms for image segmentation and pose computation (Fig. A.22), segment angle calculations (Fig. A.23) and motion control were developed in Matlab. The intended functional flow of the system was streamlined and corresponding hardware en software user interfaces and internal measurement systems were defined. Following an initial version of the graphical user interface (GUI) which contained many redundant and complicated steps (Fig. A.24), a software flowchart (Fig. A.25) and a new layout were designed and implemented to reduce the number of steps to be executed by the user to a minimum.

Figure A.26 depicts the evolution of the CAD model of the system from 2012 to 2014, driven by the knowledge obtained from the various analyses, calculations and experiments. Towards the end of 2014, the detail design of the main system and control box were completed, 2D drawings of the parts to be manufactured were made (Fig. A.27) and orders were placed. Including tooling, the system consists of over 65 subassemblies and over 650 parts, of which over 270 are different and over 190 are custom made. 


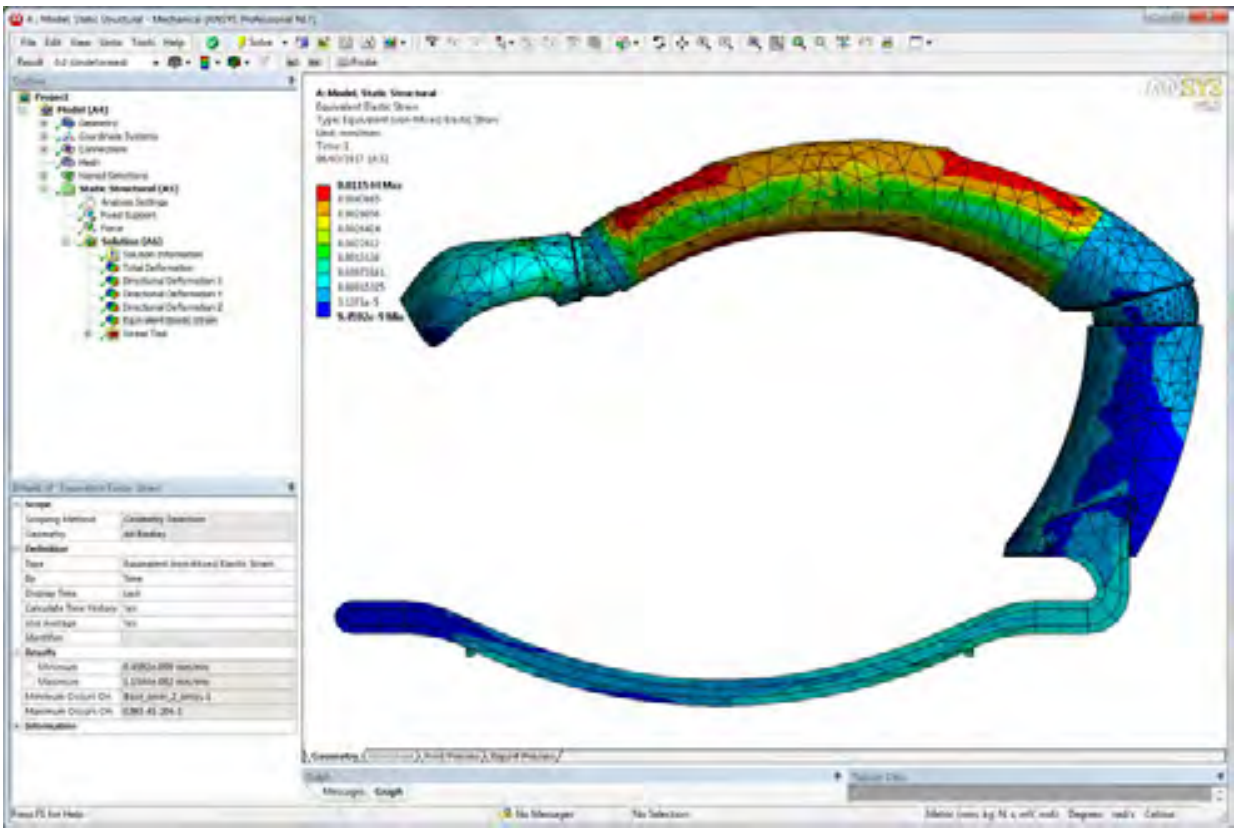

Figure A.21: Static structural analysis of the LM using Ansys (2014).

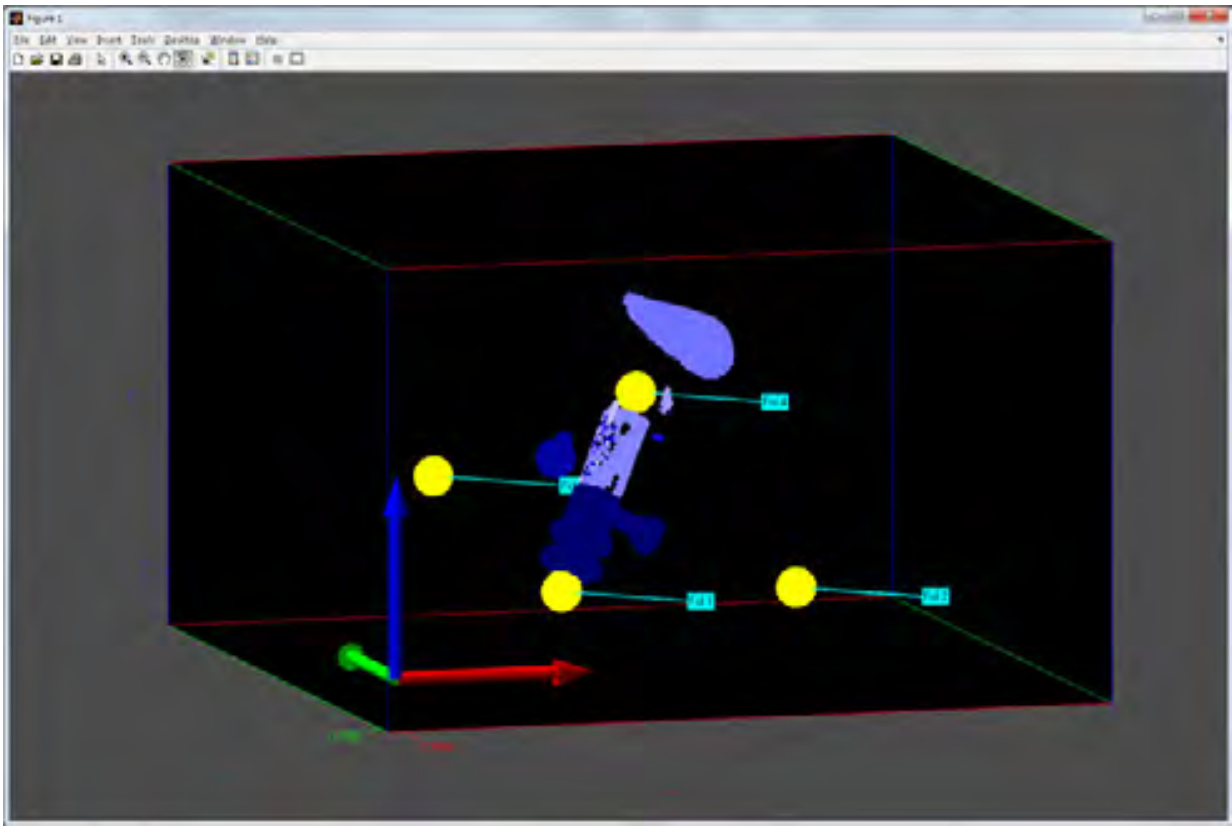

Figure A.22: 3D CT image segmentation and pose computation (2012-2016). 


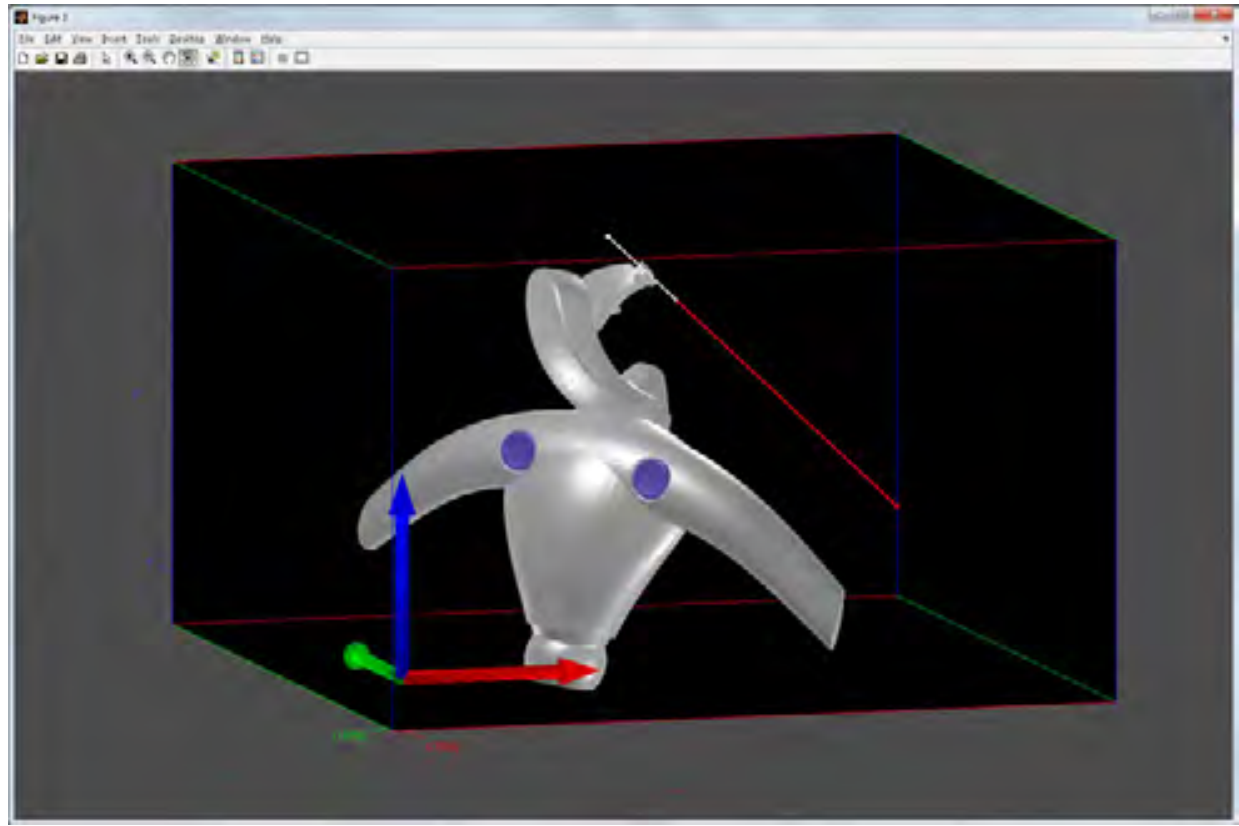

Figure A.23: Automatic calculation of the segment angles (2012-2015).

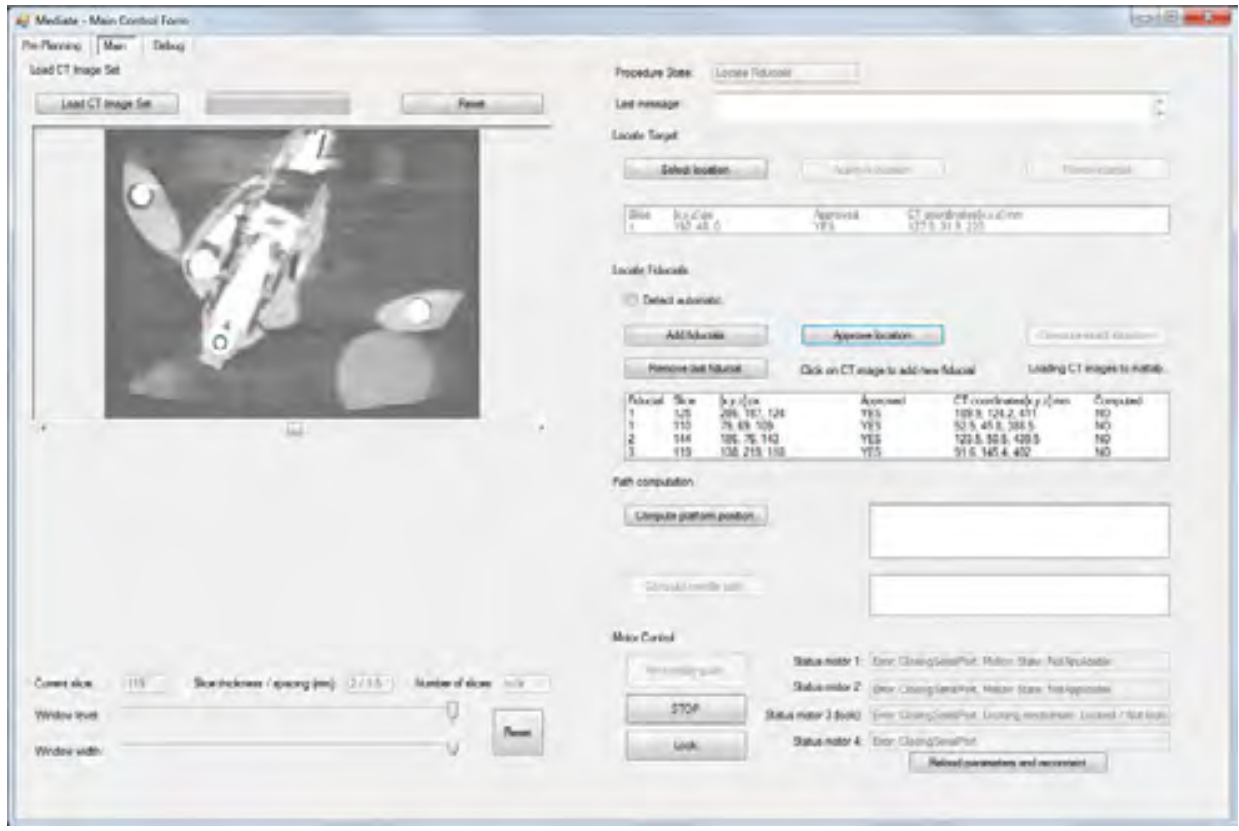

Figure A.24: Initial version of the GUI (2012). 


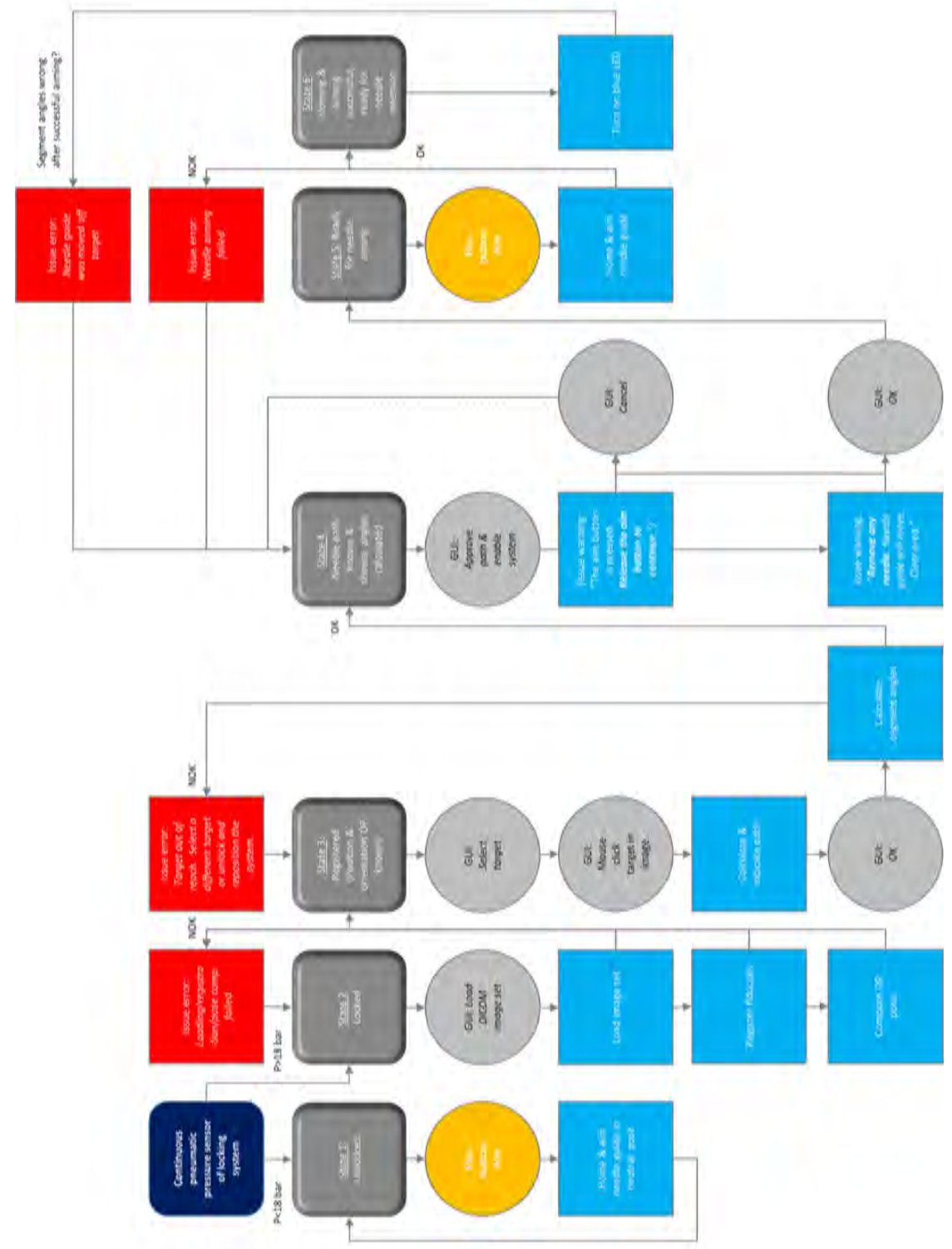

Figure A.25: Software functional flowchart (2014-2015). 


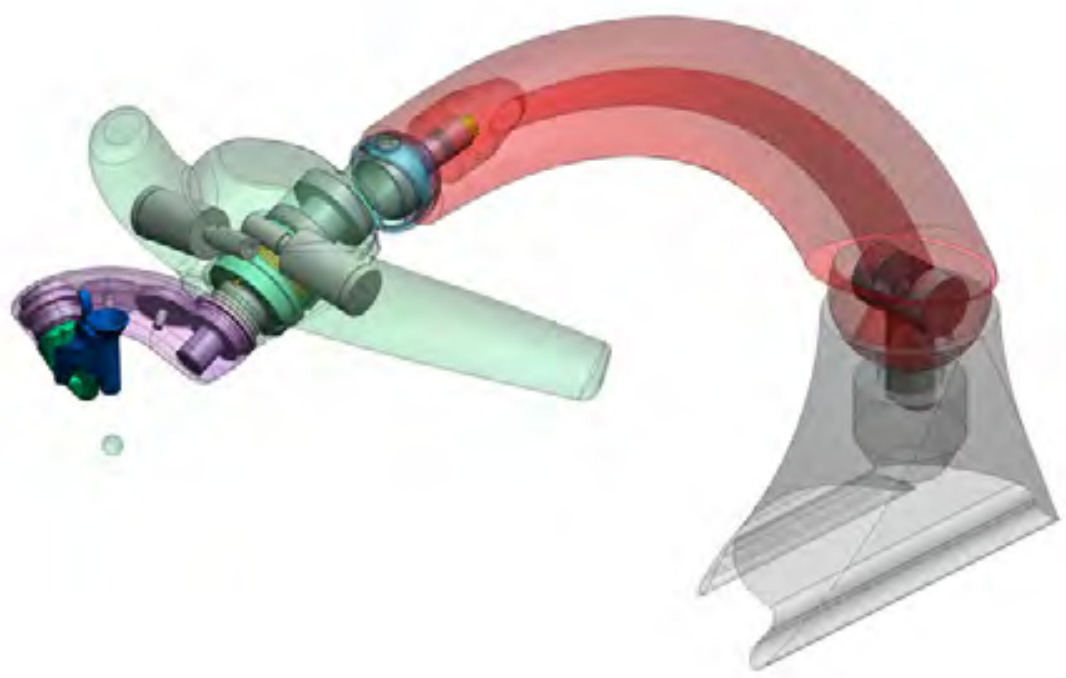

(a) Early design with actuators in the OM main body and four bevel gears to drive segment 2 (2012),

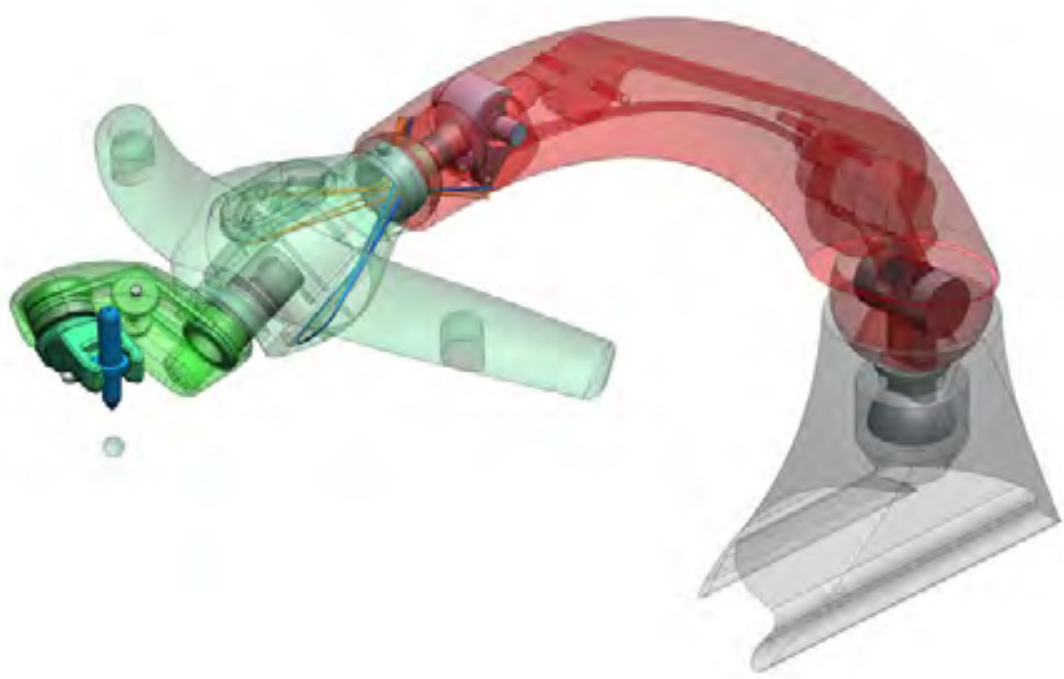

(b) Exploratory design of remote actuation of the segments using endless cable drives, locking mechanism of the ball joints, and cable drive in segment 1 to segment 2 (2012).

Figure A.26: Evolution of the CAD model (2012-2014). 


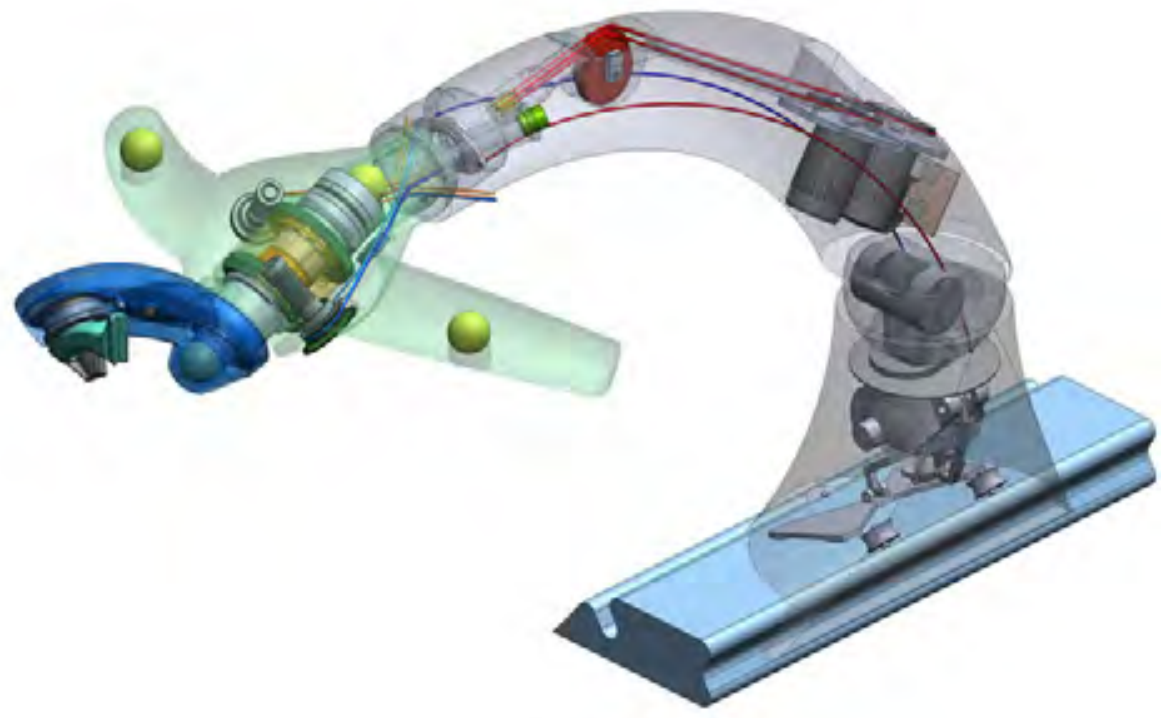

(c) Elaboration of the endless cable drives and mechanical locking system (2013).

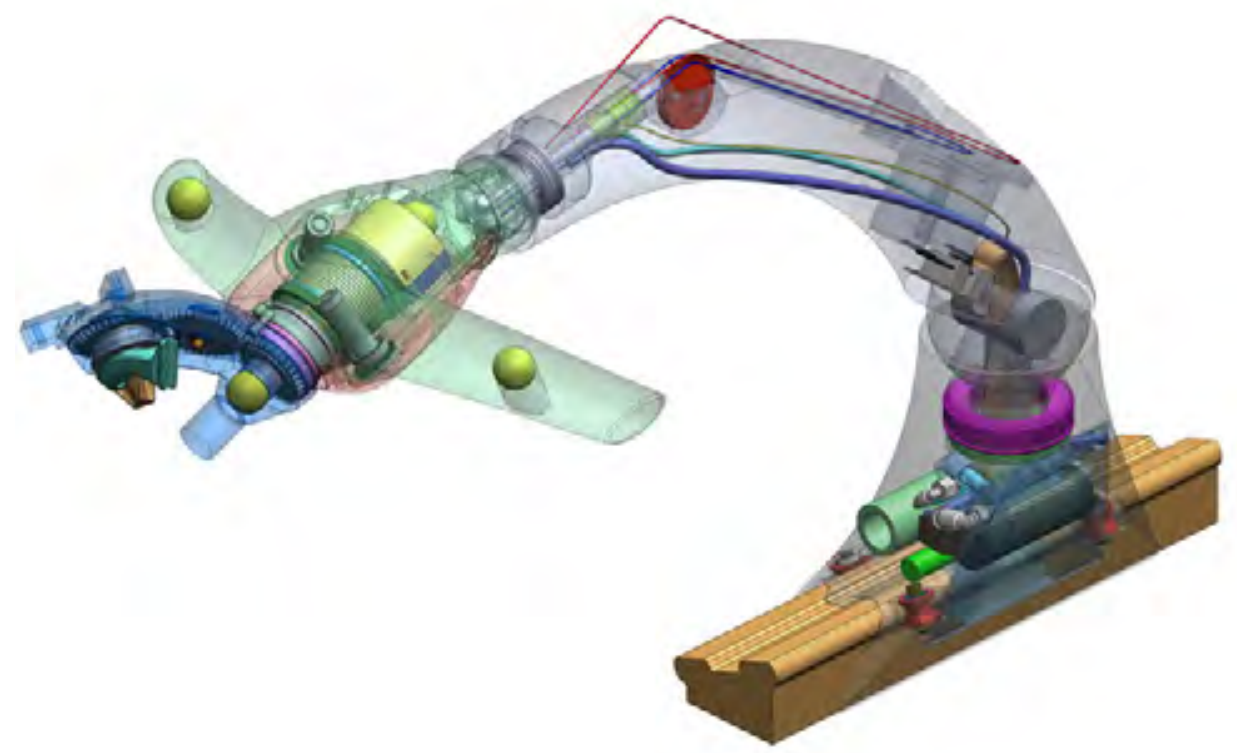

(d) Converged design of the OM and early design of the pneumatic-hydraulic locking system in the LM foot (2013).

Figure A.26: Evolution of the CAD model (2012-2014) - continued. 


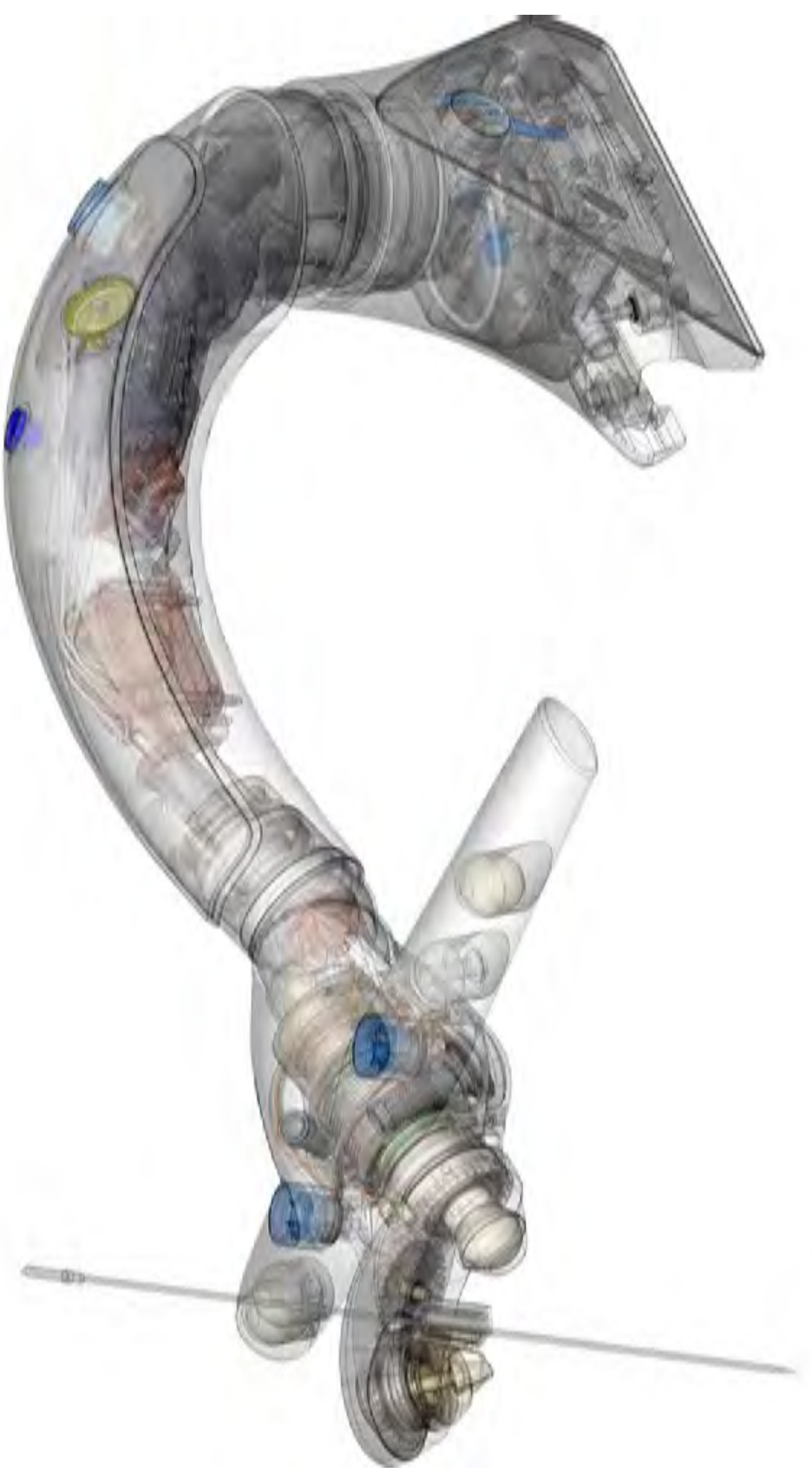

(e) Completed design (2014).

Figure A.26: Evolution of the CAD model (2012-2014) - continued. 

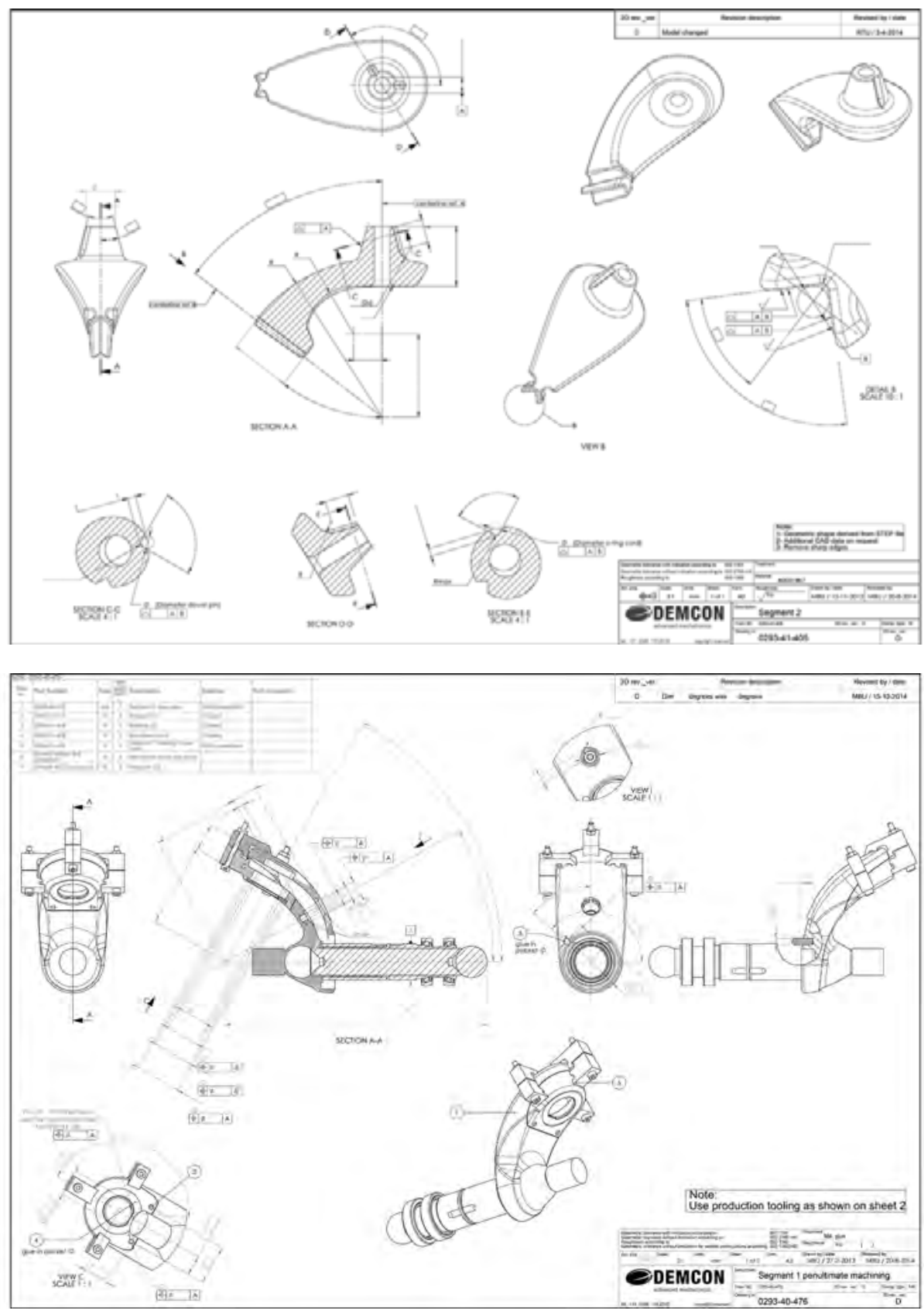

Figure A.27: Exemplary 2D drawings (2014). 

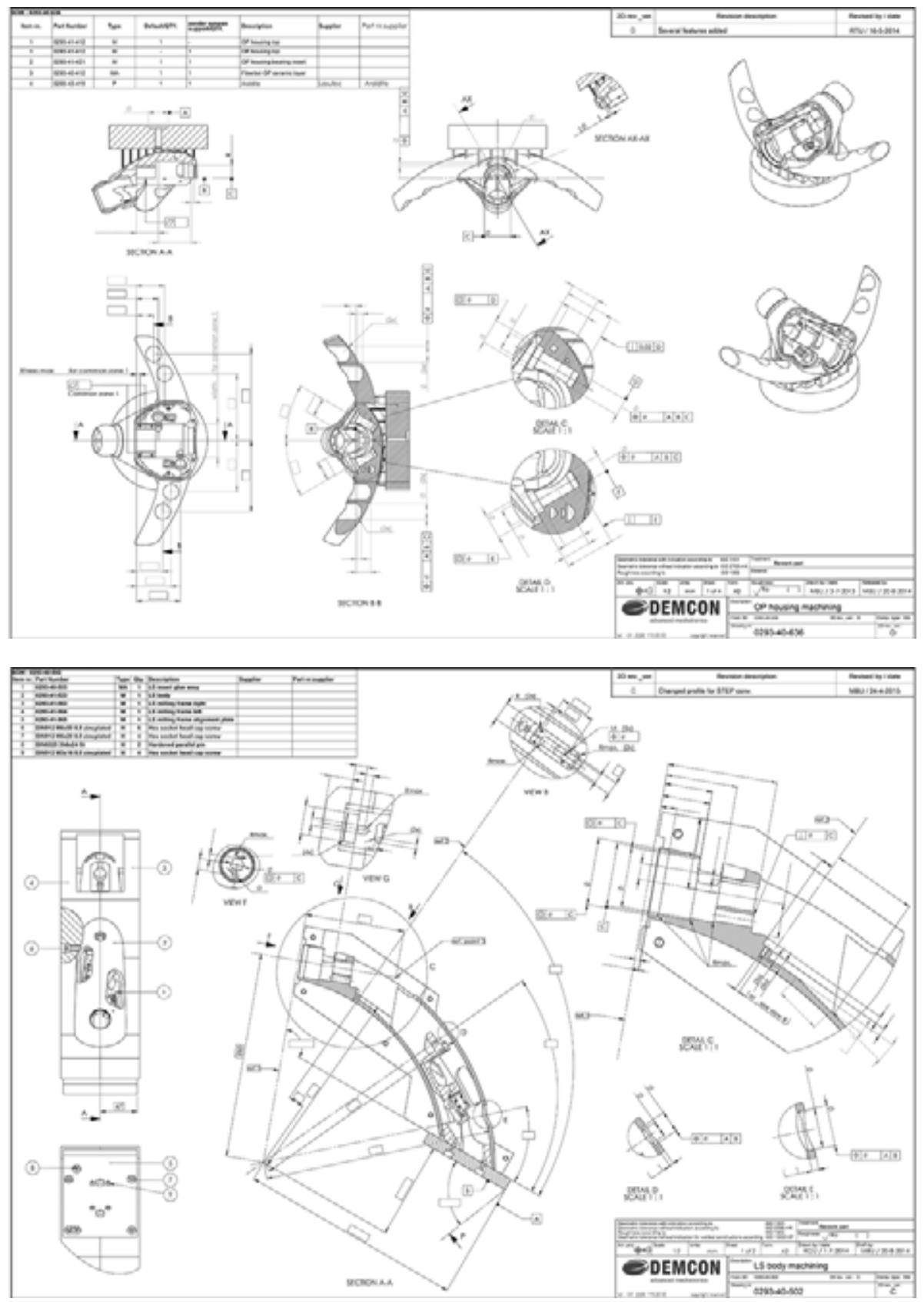

Figure A.27: Exemplary 2D drawings (2014) - continued. 

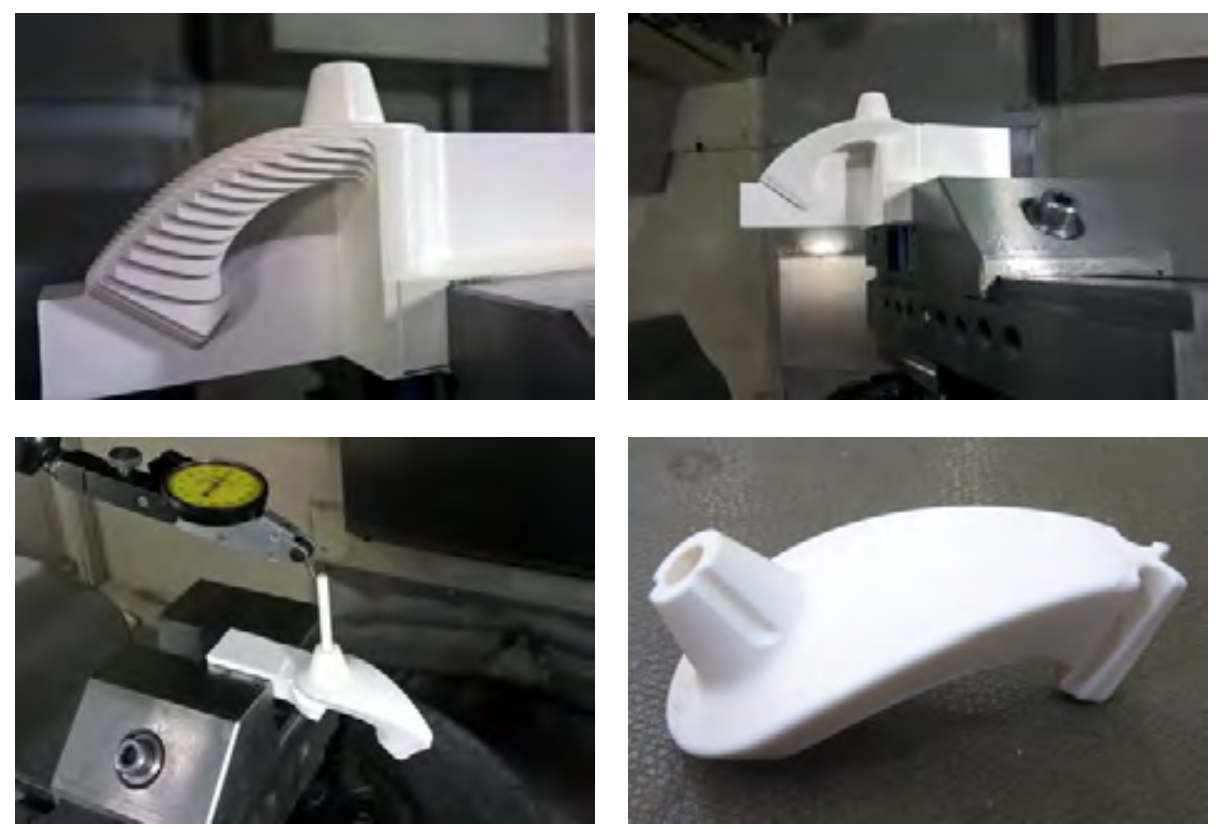

Figure A.28: Production of the aluminium oxide segment 2 by milling, sintering and precision grinding by Ceratec Technical Ceramics (2015).

Early 2015, the realization of a prototype of the completed design started. Figures A.28 to A.37 illustrate the subsequent steps of manufacture and assembly. Figure A.38 illustrates the preliminary assembly of the complete prototype. 


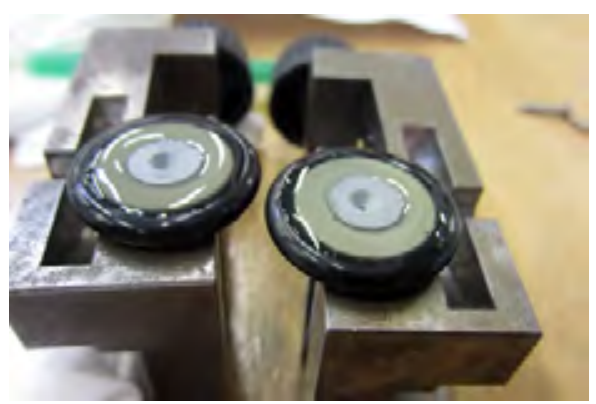

(a) Glueing O-rings to rubberise the integrated pulleys, to be grinded afterwards.

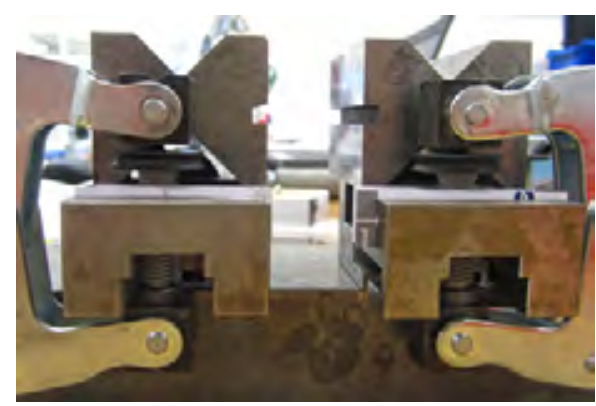

(b) Glueing silicon nitride balls on top as axial bearings.

Figure A.29: Realization of the silicon nitride worms (2015).

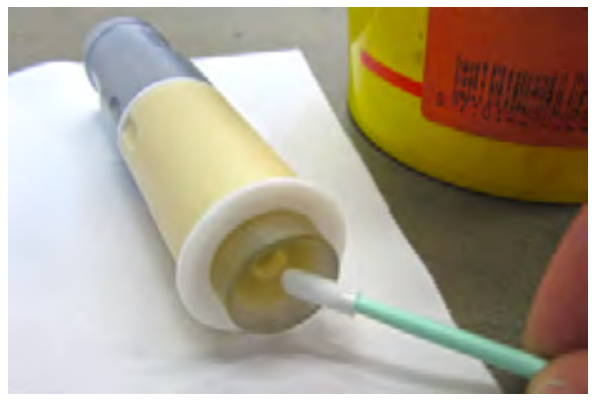

(a) Segment 1 axle assembly.

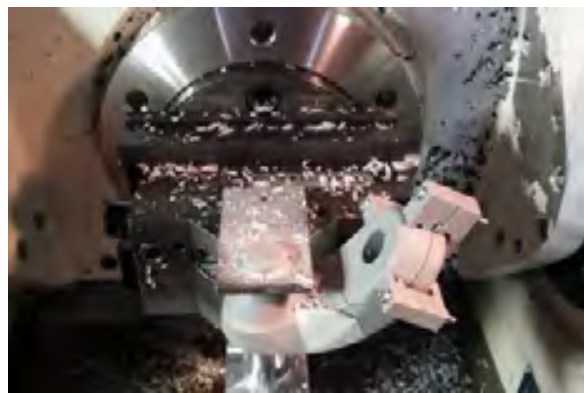

(c) Milling segment 1 by Parts \& Tools.

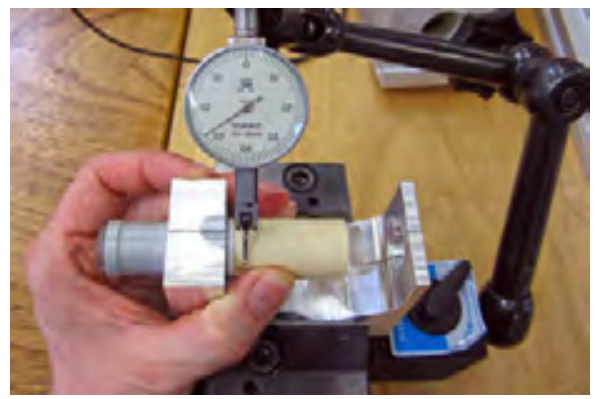

(b) Measuring the axle eccentricities.

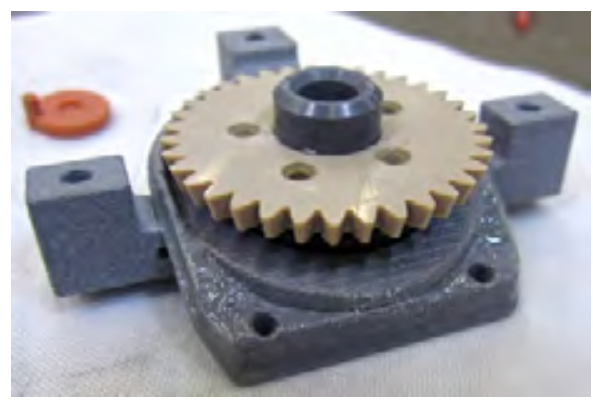

(d) Cap of segment 1 , containing the cone and bevel gear to drive segment 2 .

Figure A.30: Realization of the segment 1 subassembly (2015). 


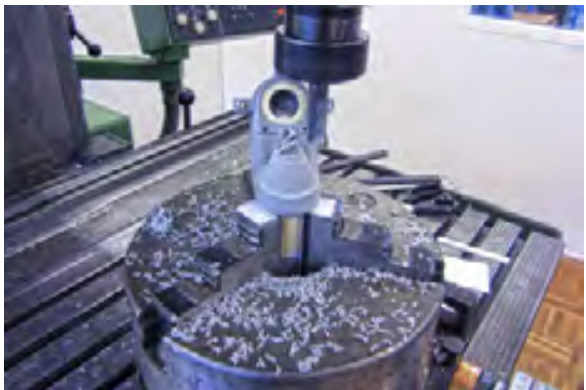

(e) Continued milling.

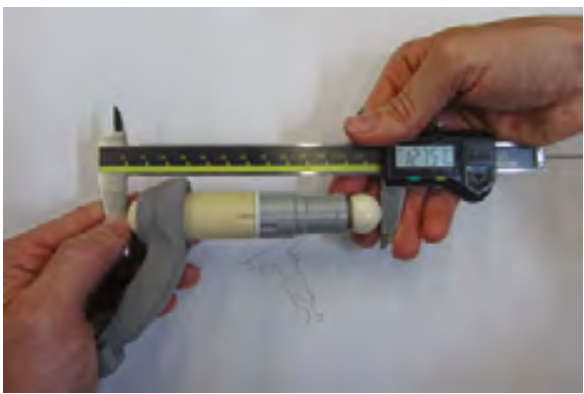

(g) Measuring the distance between two fiducial markers glued onto the axle.

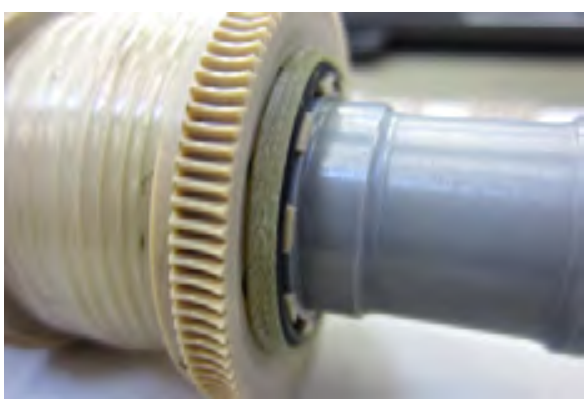

(i) Glass fibre torsion spring, worm wheel and slipper clutch after assembly.

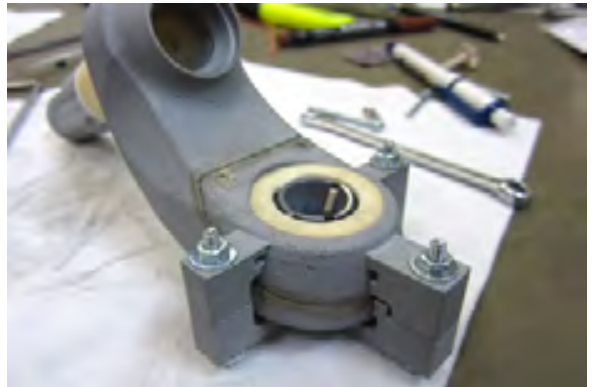

(f) Preliminary assembly of segment 1 .

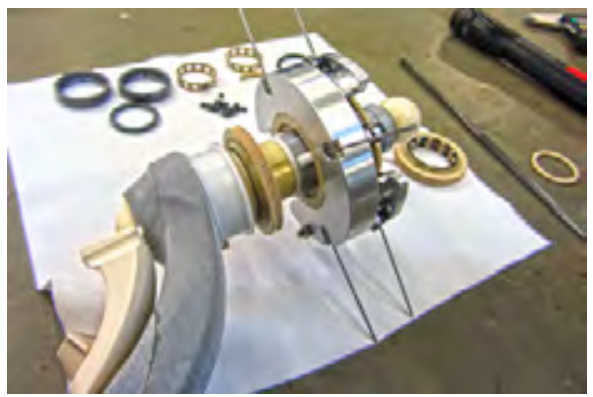

(h) Installing the glass fibre torsion spring under preload using a mounting tool.

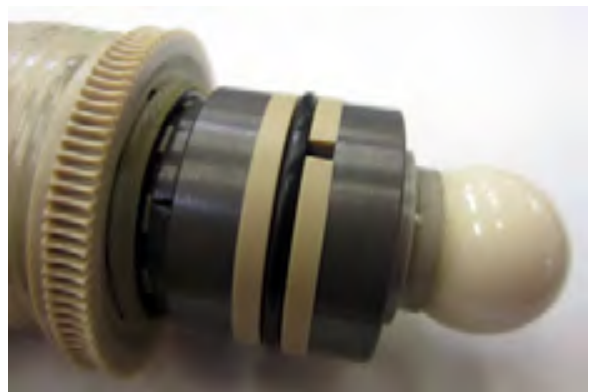

(j) Assembly of the ceramic ball bearings.

Figure A.30: Realization of the segment 1 subassembly (2015) - continued. 


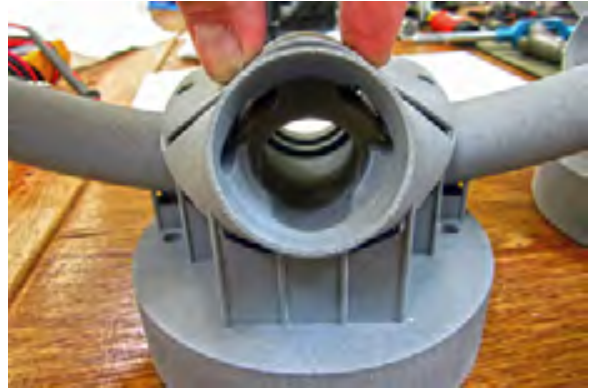

(a) Fitting the bottom cap.

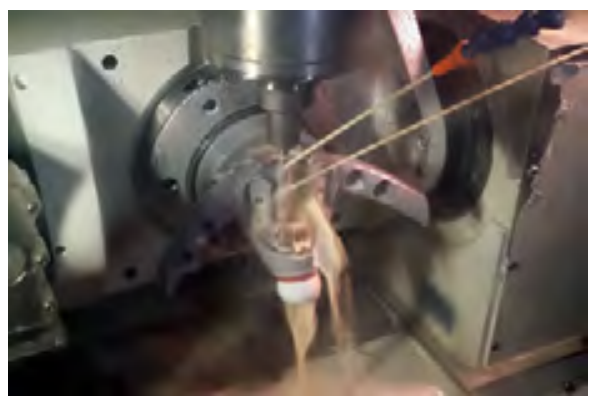

(c) Milling the OM main body by Parts \& Tools.

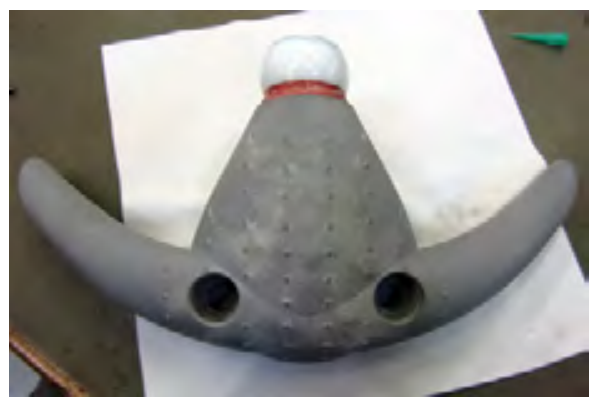

(e) Removal of the mounting base after milling.

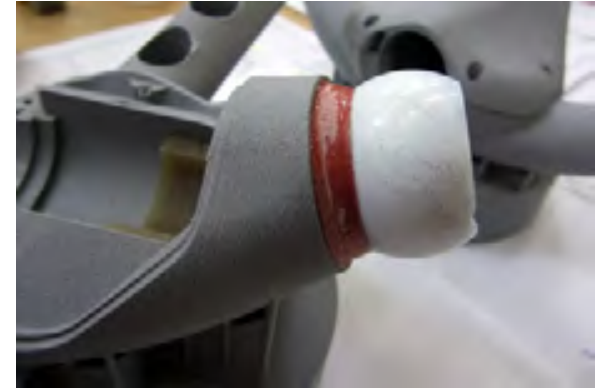

(b) Glueing ball joint 2 in place.

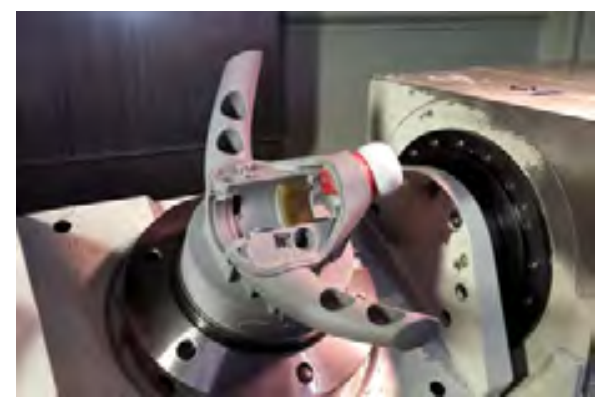

(d) Milling finished.

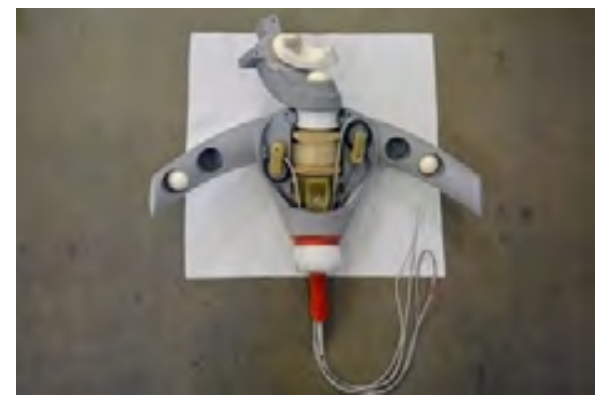

(f) Preliminary subassembly.

Figure A.31: Realization of the OM subassembly (2015). 


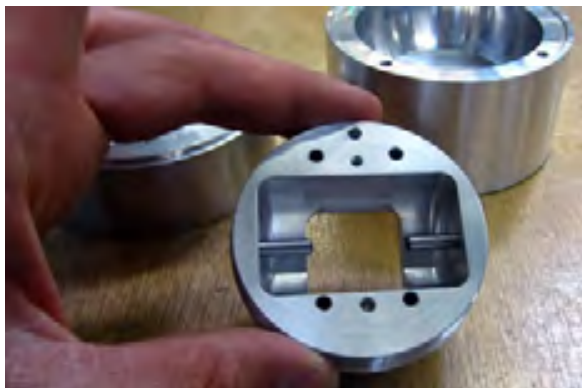

(a) Aluminium core of ball joint 1 ball and mould to cast a composite surface layer.

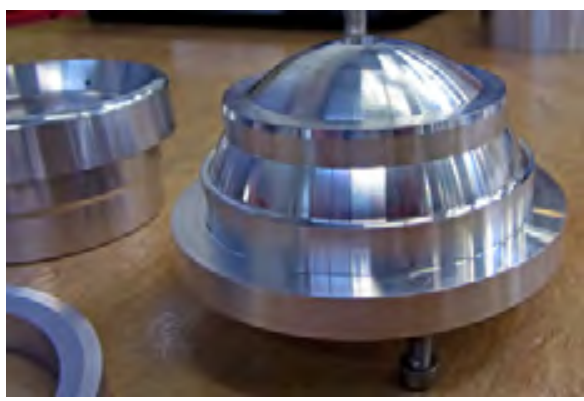

(c) Mould of ball joint 1 cup.

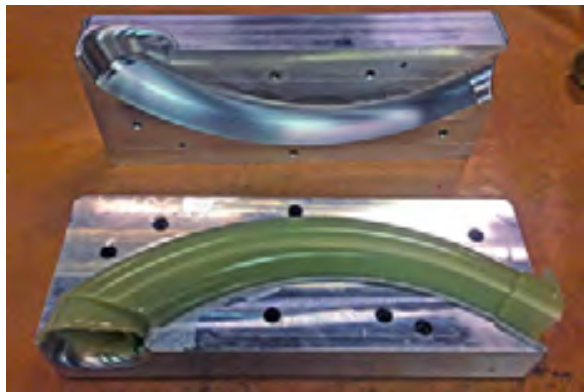

(e) Glass fibre reinforced epoxy LM arm cast by Futura Composites.

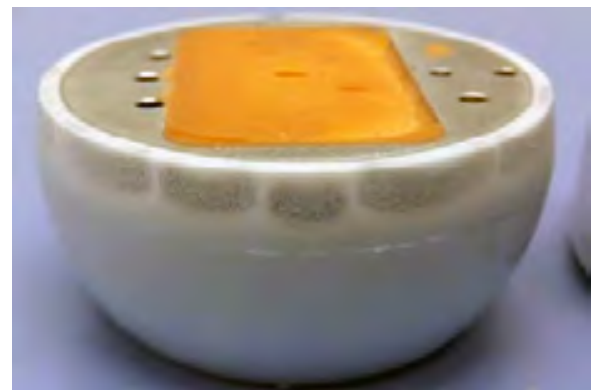

(b) Ball joint 1 ball after a first attempt to cast a composite surface layer by Futura Composites.

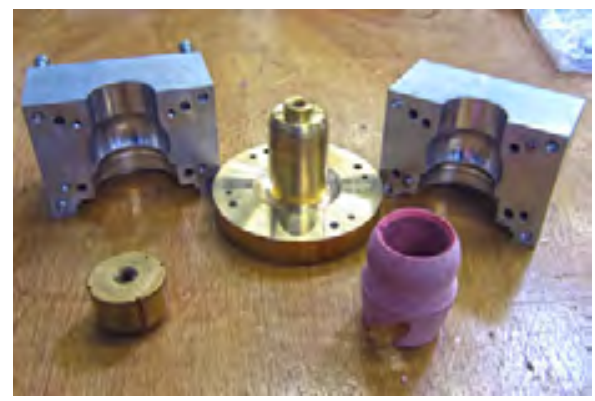

(d) Fibre reinforced core and mould of ball joint 2 ball.

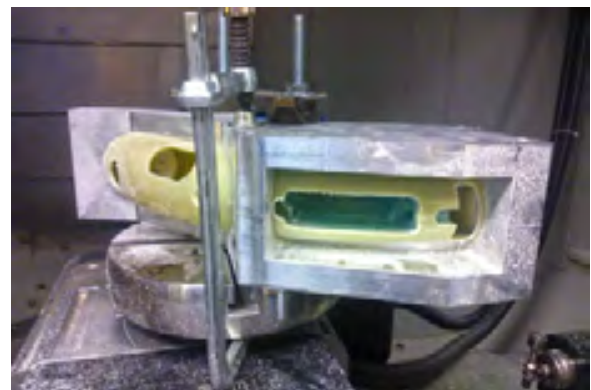

(f) Milling the LM arm in a dedicated frame.

Figure A.32: Realization of the ball joints and LM arm (2015). 


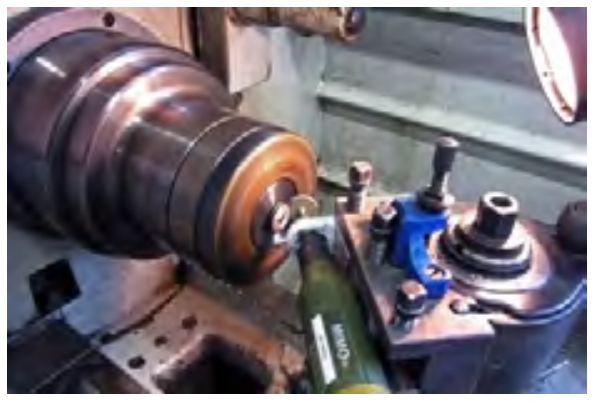

(a) Grinding the rubberised pulleys of the cable drive.

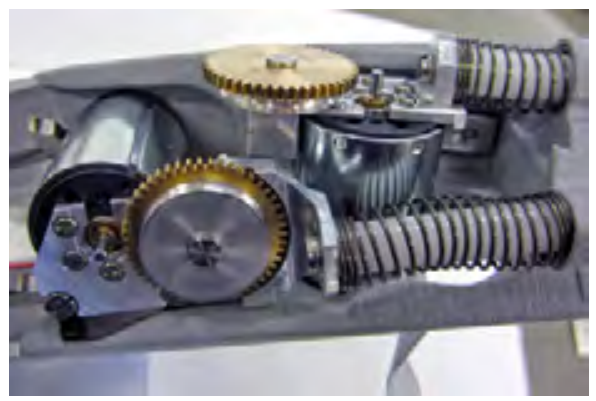

(c) Preliminary installation of the motors, spur gears and cable drive tension springs.

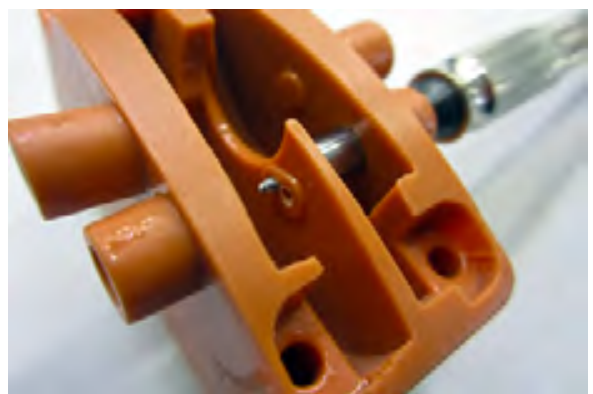

(e) Post-processing of the DLP printed pulley block part of the cable drive.

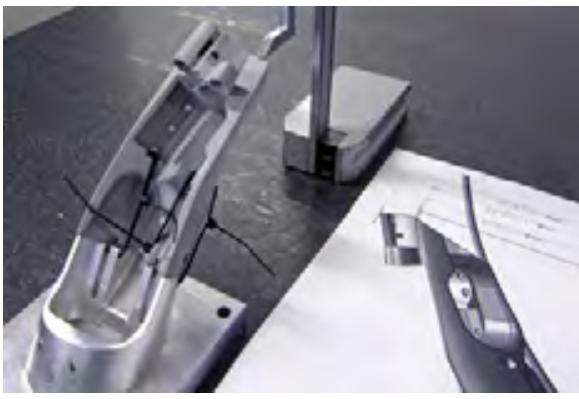

(b) Glueing the SLS printed motorframe to the aluminium bottom of the LM arm.

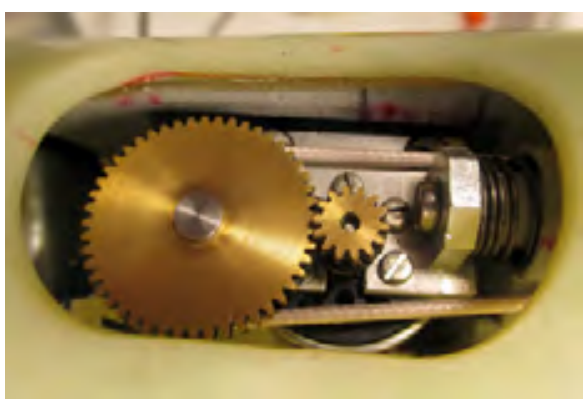

(d) Preliminary installation of a Dyneema cable.

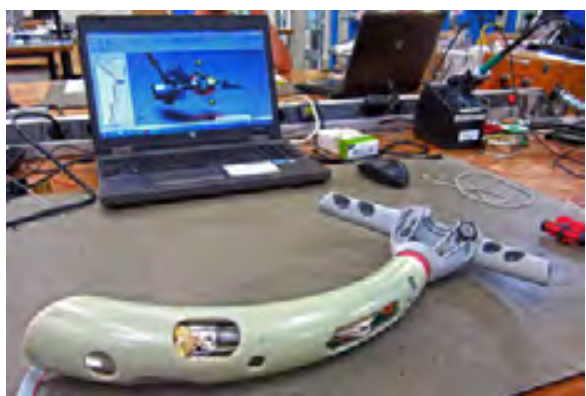

(f) Preliminary assembly of OM main body, LM arm and motor frame.

Figure A.33: Realization of the internals of the LM arm (2015). 


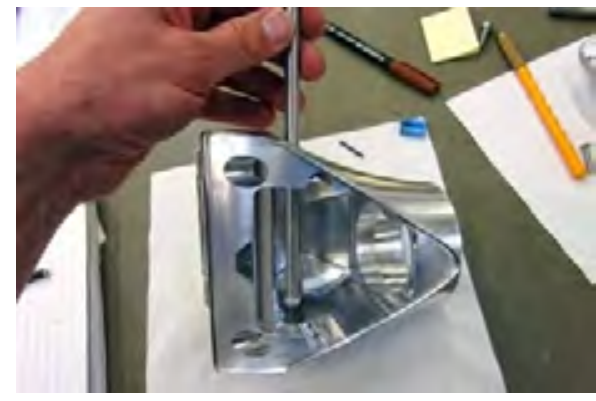

(a) LM foot main body, produced by electrical discharge machining and milling by GML Instruments.

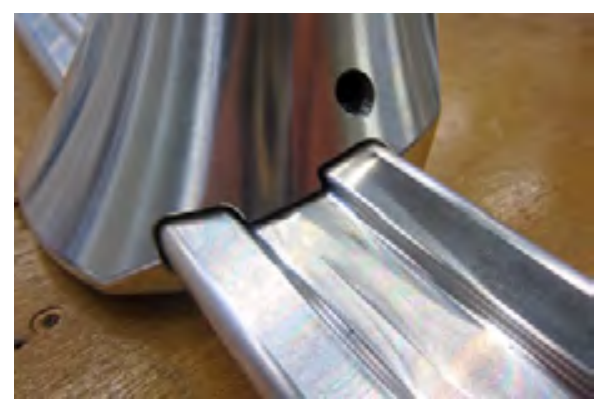

(c) Fitting the LM foot on the rail.

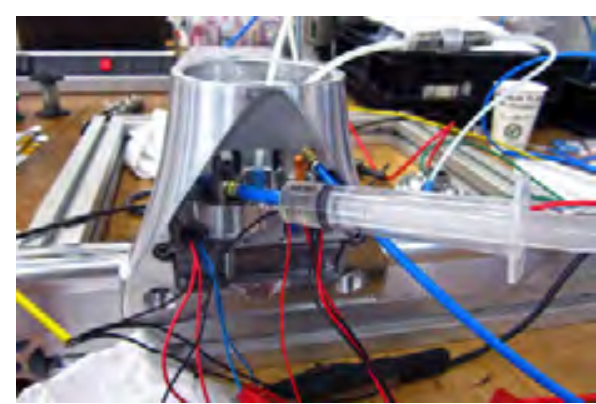

(e) Preliminary filling of the hydraulic system for testing.

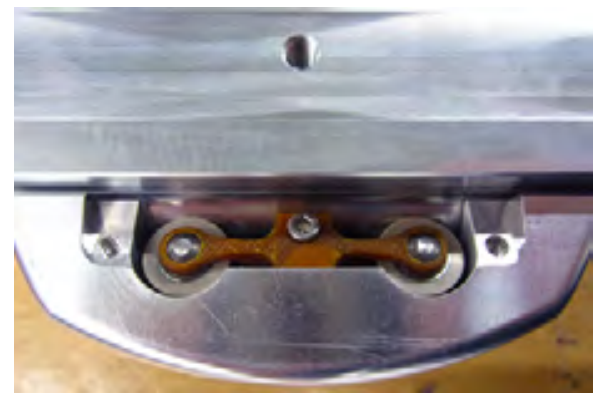

(b) Installation of the wheels of the linear slider on their glass fibre spring mount.

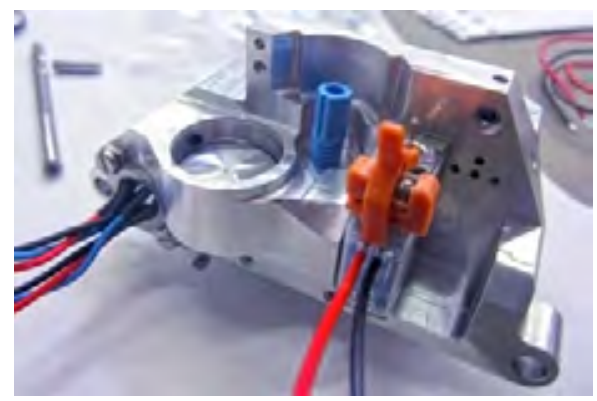

(d) Assemblying the pneumatic-hydraulic intensifier subassembly.

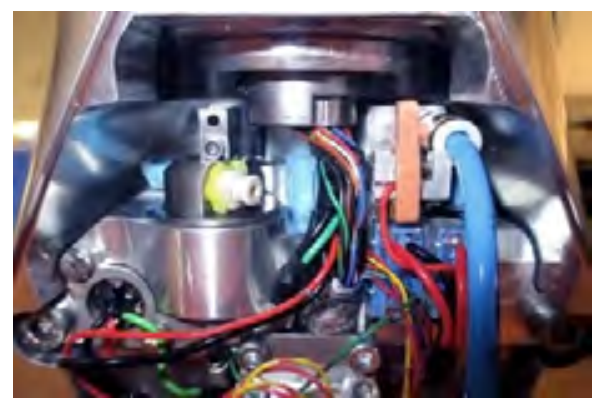

(f) Finished installation of the lower part of the pneumatic-hydraulic locking system.

Figure A.34: Realization of the LM foot subassembly (2015). 


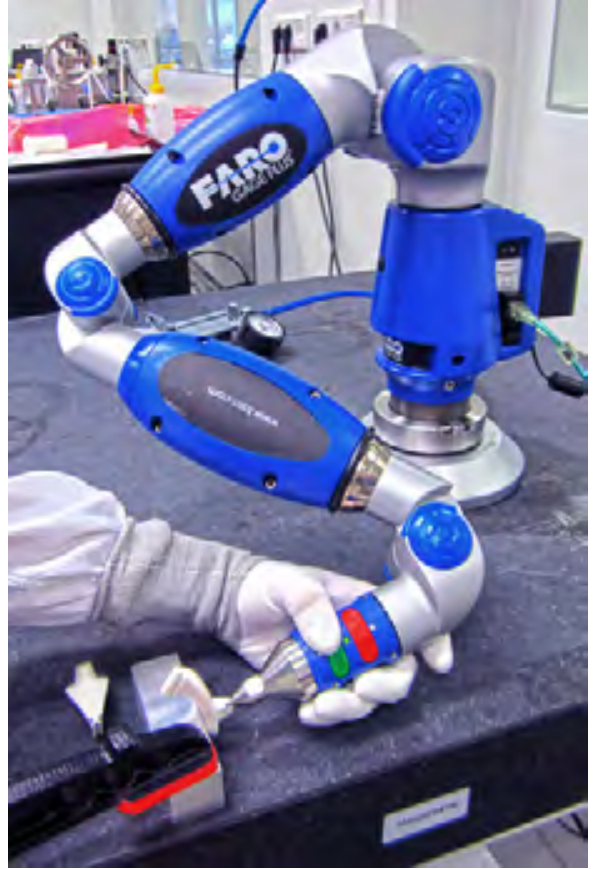

(a) Measuring segment 2.

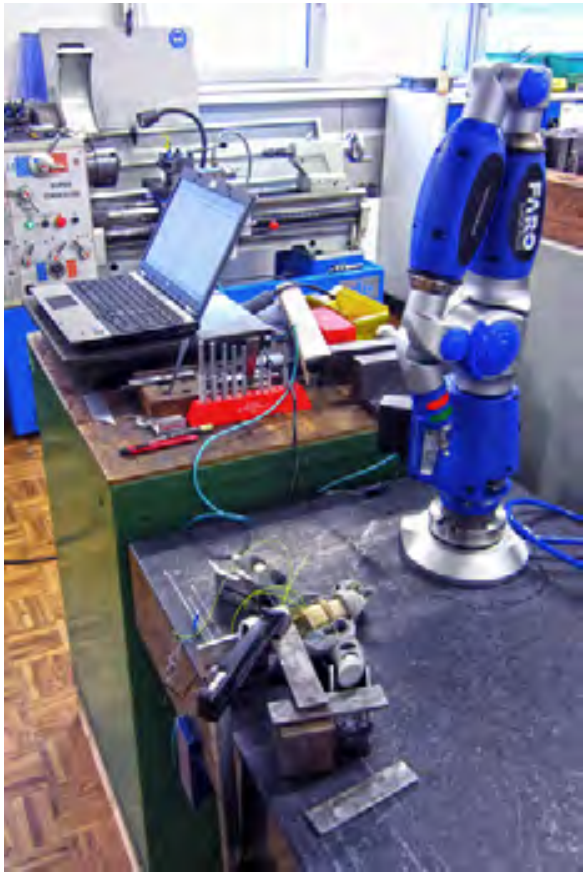

(b) Measuring the fiducial markers in the OM.

Figure A.35: Geometric measurements using a FARO Gage Plus measuring arm (2015).
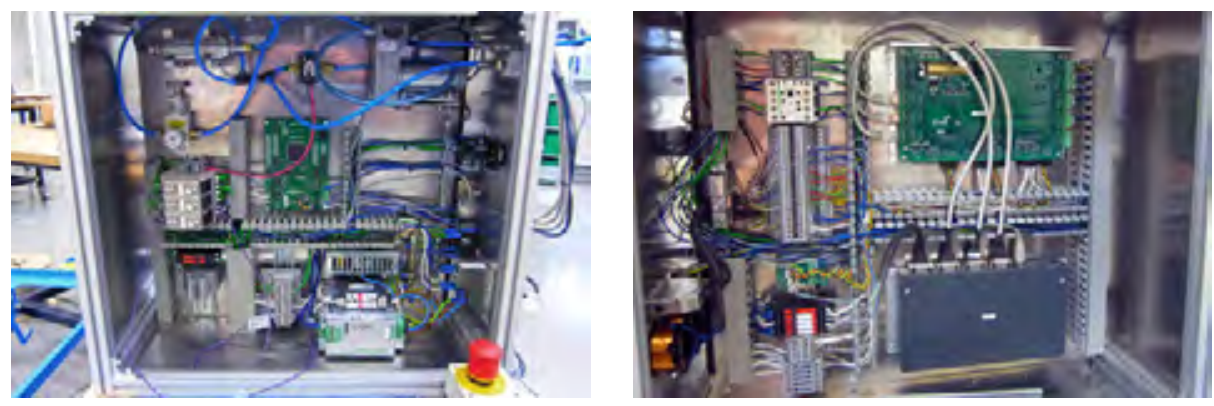

Figure A.36: Realization of the control box (2015). 

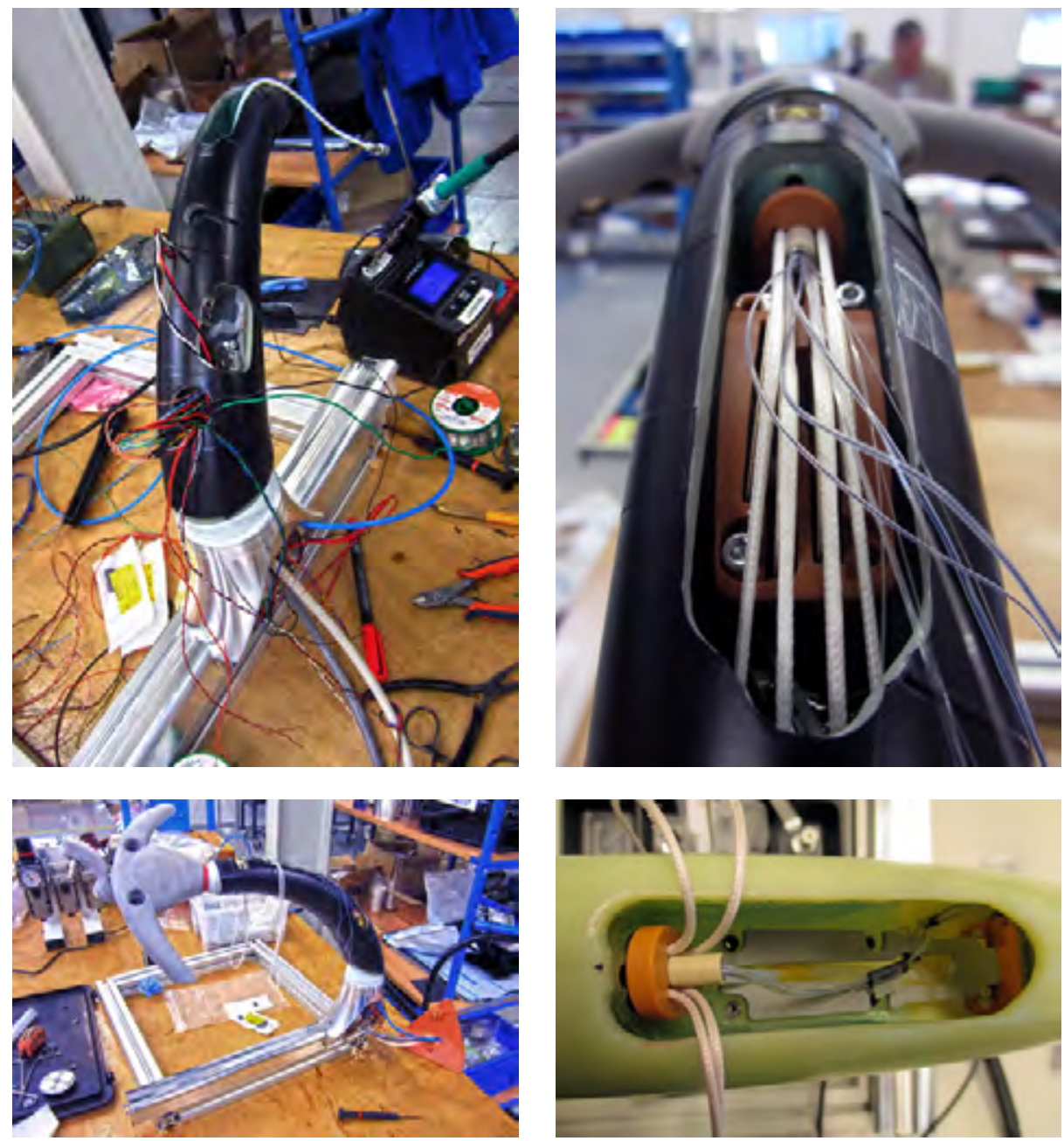

Figure A.37: Installation of the electrical wiring, optical fibres, carbon nanotubes and endless Dyneema cables (2015). 


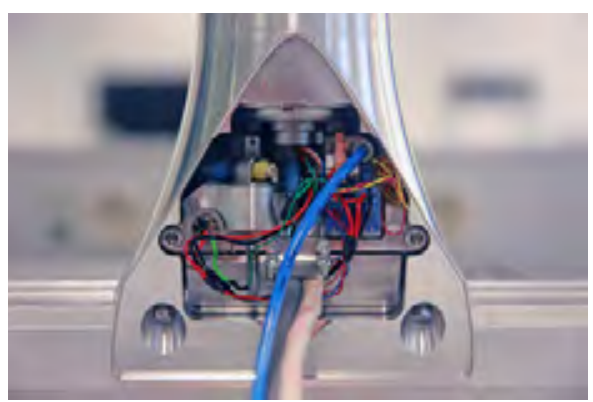

(a) LM foot and pneumatic-hydraulic locking system.

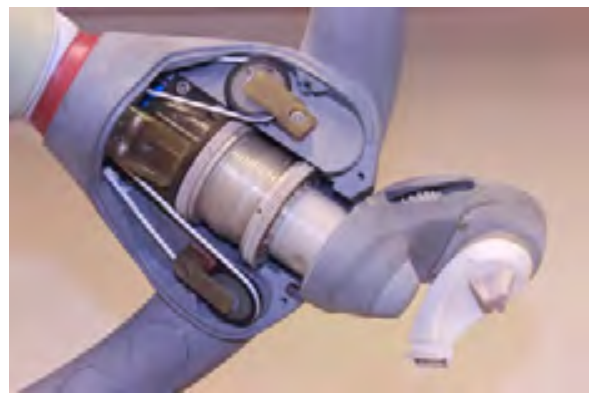

(c) Drive system of the RCM mechanism.

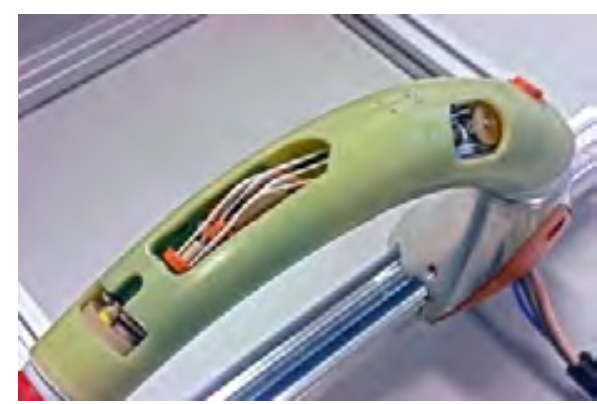

(b) LM arm and endless cable drive.

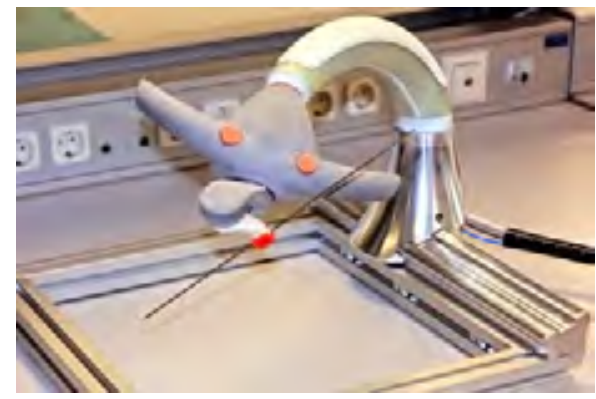

(d) Complete assembly.

Figure A.38: Preliminary assembly of the complete system (2015).

In 2015 and 2016, the prototype was integrated in the intervention suite at the UMCG (Fig. A.39), extensively tested, debugged, calibrated and technically verified (chapter 5). Minor and major modifications of the hardware and software were applied where necessary. The prototype was carefully finished with an outer layer of paint for looks and varnish for secure cleaning and disinfection. Clinical users were introduced to the system (Fig. A.40) and the system was pre-clinically validated in a phantom study (chapter 6) (Fig. A.41).

Towards clinical deployment, a dedicated CT table interface was elaborated to replace the short rail on a provisional frame of aluminium profiles (Fig. A.42). Based on measurements of the table size and shape, carbon fibre reinforced epoxy and foam sandwich constructions for a matching table plate and rail were designed to cover the full length of the table in three sections. A more economical design intended to remain outside the CT scanned volume was eventually realized, consisting of two water jet cut steel mounts matching the shape of the table and an aluminium rail of half the length of the table. 


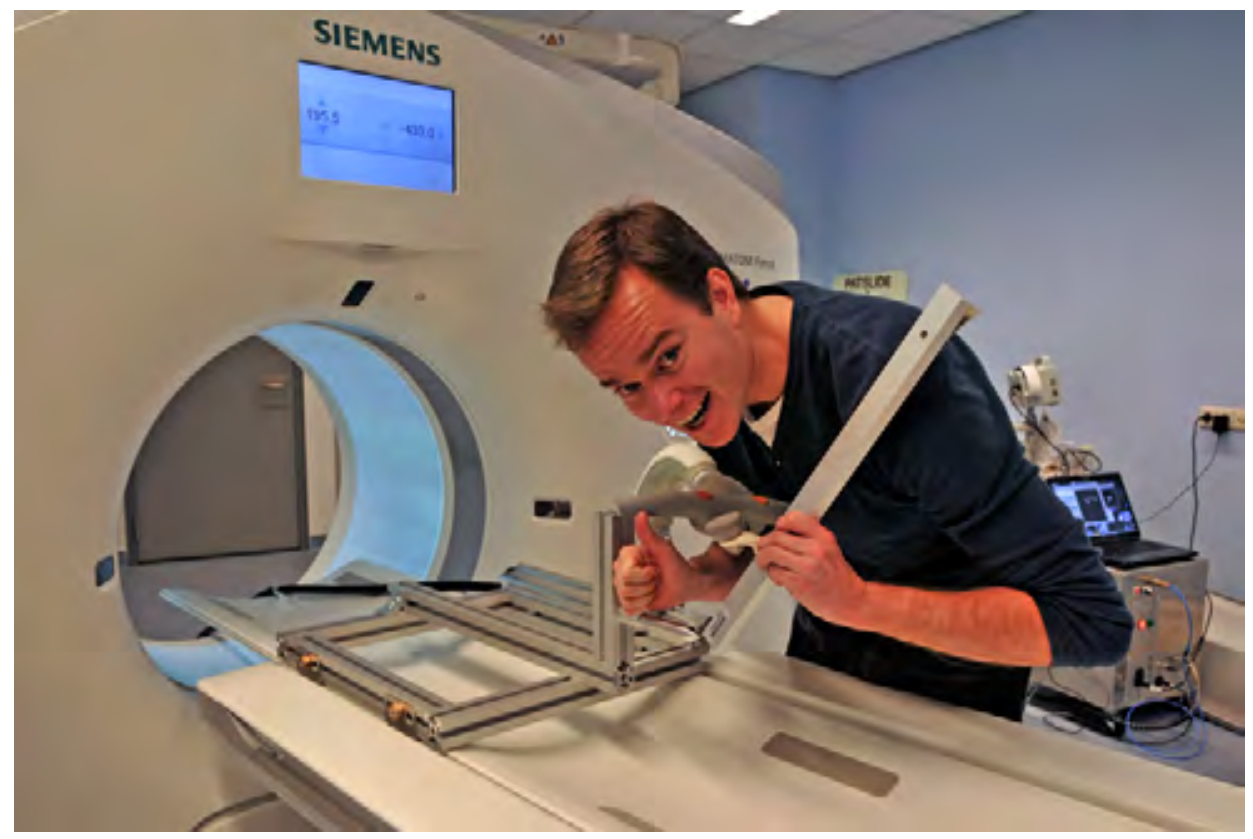

Figure A.39: The author integrating the system in the intervention suite (2015).

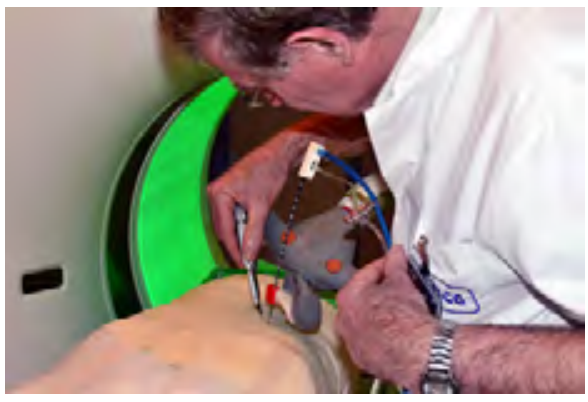

(a) Creating a skin incision at the RCM.

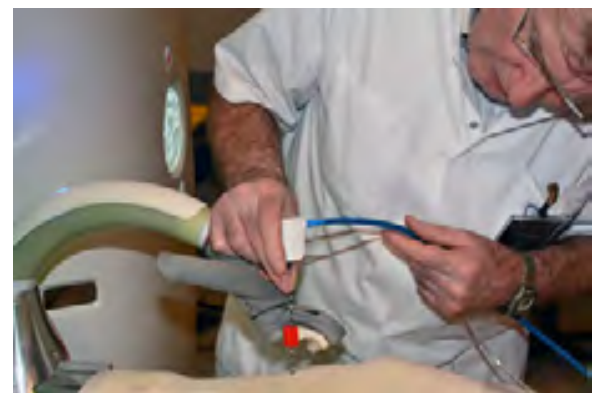

(b) Manual insertion after automated aiming.

Figure A.40: Introduction of the prototype to clinical users at the UMCG (2016). 

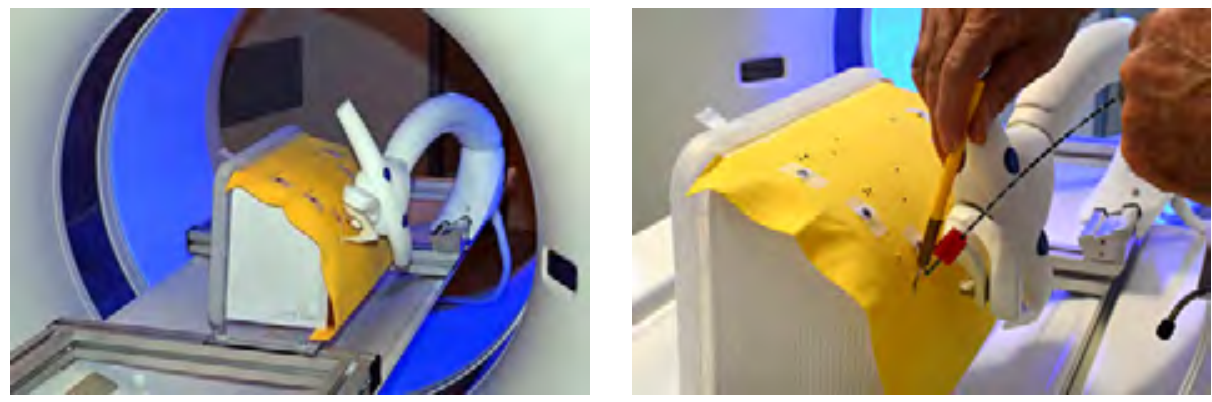

Figure A.41: Validation of the system in a phantom study (2016).

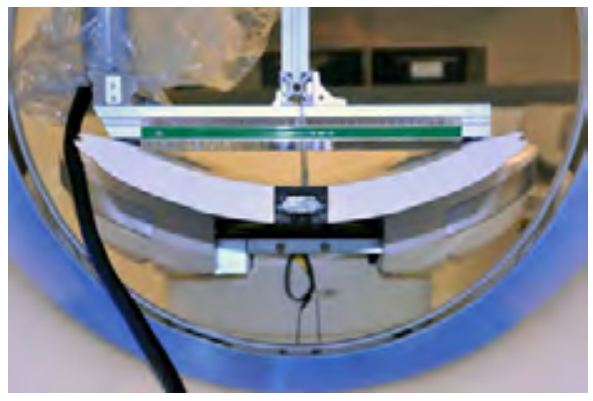

(a) Size and shape measurements of the CT table.

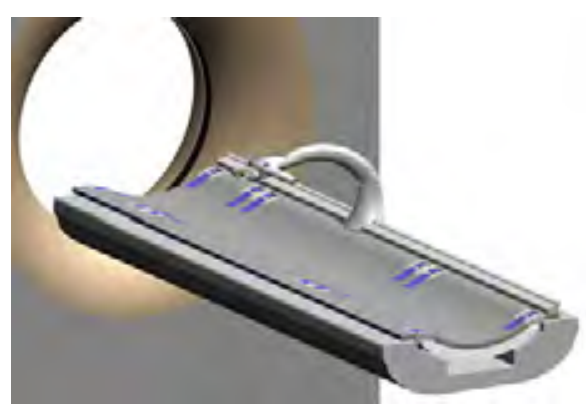

(c) Design of a full-length carbon fibre reinforced interface.

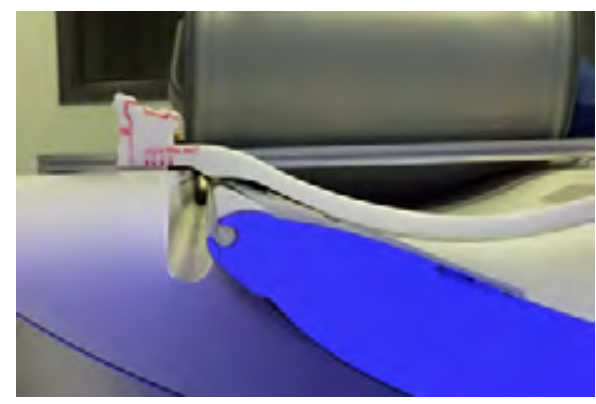

(b) Fitting a template of the interface.

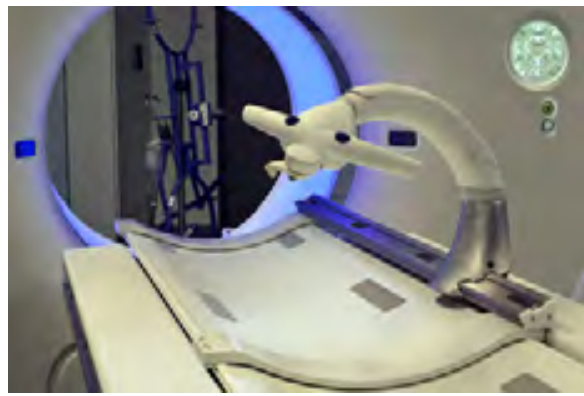

(d) Realized interface consisting of an aluminum rail on steel mounts.

Figure A.42: Development of the CT table interface (2016). 


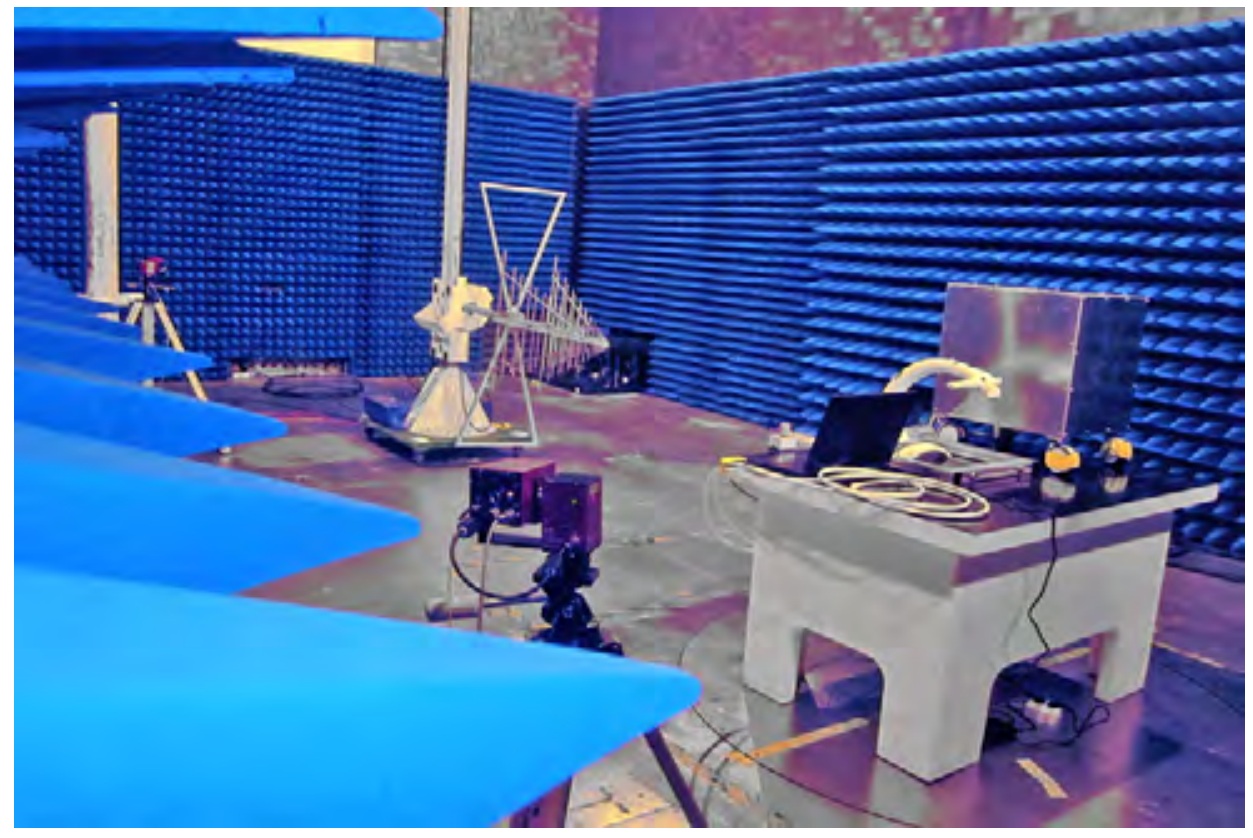

Figure A.43: EMC testing of the system at Thales (2017).

Electromagnetic compatibility (EMC) of the system is required according to EN 55011, class B. Electromagnetic emission was measured in a radio frequency anechoic chamber at the ISO/IEC 17025 L240 accredited test laboratory of the Thales Environmental Competence Centre (Fig. A.43). The results were in compliance with the applicable standard.

To obtain permission from the Medical Ethical Committee of the UMCG and the Dutch Health Care Inspectorate (IGZ) for clinical deployment of the system, we delivered a long list of signed paperwork produced over the course of the development, including the system requirements documents, concept design documents, software interface design documents, the bill of materials, the software revision level history, test and analysis reports (system, electrical and software), requirement verification reports, component sterilization instructions, declarations of suitability for sterilization, pre-clinical evidence, a user manual, user training records, the risk management file, the essential requirements checklist, the investigational medical device dossier, and the IEC 60601 checklist.

Figure A.44 shows the completed system in 2017, ready for deployment in clinical practise. 


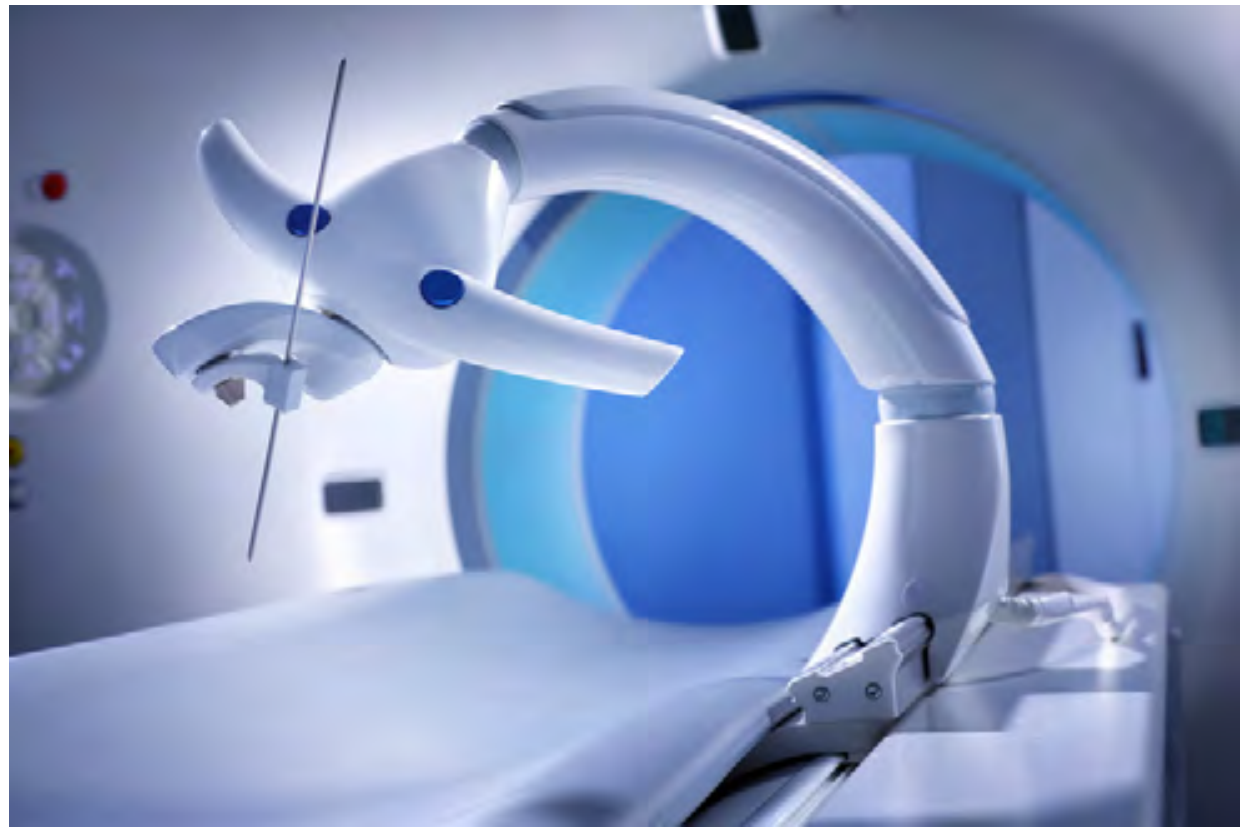

Figure A.44: The completed system in the intervention suite (2017). 


\section{References}

[1] DEMCON, Institutenweg 25, $7521 \mathrm{PH}$, Enschede, the Netherlands. https://www.demcon.nl.

[2] University of Twente, Drienerlolaan 5, 7522 NB, Enschede, the Netherlands. https:/ / www.utwente.nl.

[3] D. Patch and A.P. Dhillon. Biopsy of the liver. In J.S. Dooley, A.S. Lok, A.K. Burroughs, and E.J. Heathcote, editors, Sherlock's diseases of the liver and biliary system, chapter 3, pages 36-47. Wiley-Blackwell, Hoboken, New Jersey, USA, $12^{\text {th }}$ edition, 2011.

[4] R.S. Winokur, B.B. Pua, B.W. Sullivan, and Madoff D.C. Percutaneous lung biopsy: technique, efficacy, and complications. Seminars in Interventional Radiology, 30(2):121-127, 2013.

[5] J. Scheele, A. Altendorf-Hofmann, R. Stangl, and K. Schmidt. Surgical resection of colorectal liver metastases: gold standard for solitary and radically resectable lesions. Swiss Surgery, 4:4-17, 1996.

[6] Registry of Hepatic Metastases. Resection of the liver for colorectal carcinoma metastases: a multi-institutional study of indications for resection. Surgery, 103(3):278-288, 1988.

[7] P. Rougier, C. Milan, F. Lazorthes, G. Fourtanier, C. Partensky, H. Baumel, and J. Faivre. Prospective study of prognostic factors in patients with unresected hepatic metastases from colorectal cancer. British Journal of Surgery, 82(10):1397-1400, 1995.

[8] M.G. Hartwig and T.A. D'Amico. Thoracoscopic lobectomy: the gold standard for early-stage lung cancer? The Annals of Thoracic Surgery, 89(6):S2098-S2101, 2010.

[9] A.S. Wright, D.M. Mahvi, D.G. Haemmerich, and F.T. Lee. Minimally invasive approaches in management of hepatic tumors. Surgical Technology International, 11:144-153, 2003.

[10] A.R. Gillams. Image guided tumour ablation. Cancer Imaging, 5:103-109, 2005.

[11] M. Ahmed, C.L. Brace, F.T. Lee, and S.N. Goldberg. Principles of and advances in percutaneous ablation. Radiology, 258(2):351-369, 2011. 
[12] D. Li, J. Kang, B.J. Golas, V.W. Yeung, and D.C. Madoff. Minimally invasive local therapies for liver cancer. Cancer Biology \& Medicine, 11(4):217-236, 2014.

[13] D.E. Dupuy and M. Shulman. Current status of thermal ablation treatments for lung malignancies. Seminars in Interventional Radiology, 27(3):268-275, 2010.

[14] P.D. Sonntag, J.L. Hinshaw, M.G. Lubner, C.L. Brace, and F.T Lee. Thermal ablation of lung tumors. Surgical Oncology Clinics of North America, 20(2):369-387, 2011.

[15] Y. Robert Sheu and K. Hong. Percutaneous lung tumor ablation. Techniques in Vascular \& Interventional Radiology, 16(4):239-252, 2013.

[16] T. Schneider, C.P. Heussel, F.J.F. Herth, and H. Dienemann. Thermal ablation of malignant lung tumors. Deutsches Ärzteblatt International, 110(22):394-400, 2013.

[17] E.S. Alexander and D.E. Dupuy. Lung cancer ablation: technologies and techniques. Seminars in Interventional Radiology, 30(2):141-150, 2013.

[18] Q.Z. Al-Tariq. Percutaneous strategies for the management of pulmonary parenchymal, chest wall, and pleural metastases. American Journal of Roentgenology, 203(4):709-716, 2014.

[19] L.A. Torre, F. Bray, R.L. Siegel, J. Ferlay, J. Lortet-Tieulent, and A. Jemal. Global cancer statistics, 2012. CA: A Cancer Journal for Clinicians, 65(2):87108, 2015.

[20] W.T. London and McGlynn K.A. Liver cancer. In D. Schottenfeld and J.F. Fraumeni, Jr., editors, Cancer Epidemiology and Prevention, chapter 39, pages 763-786. Oxford University Press, New York City, New York, USA, $3^{\text {rd }}$ edition, 2006.

[21] J.G. Geoghegan and J. Scheele. Treatment of colorectal liver metastases. British Journal of Surgery, 86(2):158-169, 1999.

[22] S.B. Solomon and S.G. Silverman. Imaging in interventional oncology. Radiology, 257(3):624-640, 2010.

[23] M. Ahmed, L. Solbiati, C.L. Brace, D.J. Breen, M.R. Callstrom, J.W. Charboneau, M. Chen, B. Ihn Choi, T. de Baère, G.D. Dodd, III, D.E. Dupuy, D.A. Gervais, D. Gianfelice, A.R. Gillams, F.T. Lee, Jr., E. Leen, R. Lencioni, P.J. Littrup, T. Livraghi, D.S. Lu, J.P. McGahan, M. Franca Meloni, B. Nikolic, P.L. Pereira, P. Liang, H. Rhim, S.C. Rose, R. Salem, C.T. Sofocleous, S.B. Solomon, M.C. Soulen, M. Tanaka, T.J. Vogl, B.J. Wood, and S.N. Goldberg. Image-guided tumor ablation: standardization of terminology and reporting criteria - a 10-year update. Radiology, 273(1):241-260, 2014. 
[24] D.S. Memel, G.D. Dodd, and C.C. Esola. Efficacy of sonography as a guidance technique for biopsy of abdominal, pelvic, and retroperitoneal lymph nodes. American Journal of Roentgenology, 167(4):957-962, 1996.

[25] L. Appelbaum, R.A. Kane, J.B. Kruskal, J. Romero, and J. Sosna. Focal hepatic lesions: US-guided biopsy - lessons from review of cytologic and pathologic examination results. Radiology, 250(2):453-458, 2009.

[26] G. Vijayaraghavan, S. David, M. Bermudez-Allende, and H. Sarwat. Imaging-guided parenchymal liver biopsy: how we do it. Journal of Clinical Imaging Science, 1(1):30, 2011.

[27] S. Rossi, M. Di Stasi, E. Buscarini, P. Quaretti, F. Garbagnati, L. Squassante, C.T. Paties, D.E. Silverman, and L. Buscarini. Percutaneous RF interstitial thermal ablation in the treatment of hepatic cancer. American Journal of Roentgenology, 167(3):759-768, 1996.

[28] S.A. Curley, F. Izzo, P. Delrio, L.M. Ellis, J. Granchi, P. Vallone, F. Fiore, S. Pignata, B. Daniele, and F. Cremona. Radiofrequency ablation of unresectable primary and metastatic hepatic malignancies: results in 123 patients. Annals of Surgery, 280(1):1-8, 1999.

[29] L. Solbiati, T. Livraghi, S.N. Goldberg, T. Ierace, F. Meloni, M. Dellanoce, L. Cova, E.F. Halpern, and G.S. Gazelle. Percutaneous radio-frequency ablation of hepatic metastases from colorectal cancer: long-term results in 117 patients. Radiology, 221(1):159-166, 2001.

[30] R. Lencioni, D. Cioni, L. Crocetti, C. Franchini, C. Della Pina, J. Lera, and C. Bartolozzi. Early-stage hepatocellular carcinoma in patients with cirrhosis: long-term results of percutaneous image-guided radiofrequency ablation. Radiology, 234(3):961-967, 2005.

[31] L. Solbiati, M. Ahmed, L. Cova, T. Ierace, M. Brioschi, and S.N. Goldberg. Small liver colorectal metastases treated with percutaneous radiofrequency ablation: local response rate and long-term survival with up to 10-year follow-up. Radiology, 265(3):958-968, 2012.

[32] S.R. Wilson, L.D. Greenbaum, and B.B. Goldberg. Contrast-enhanced ultrasound: what is the evidence and what are the obstacles? American Journal of Roentgenology, 193(1):55-60, 2009.

[33] Bracco Diagnostics Inc. First Approval by U.S. Food and Drug Administration for contrast enhanced ultrasonography of the liver received by Bracco Diagnostics Inc. for LUMASON@ (sulfur hexafluoride lipid-type A microspheres) for injectable suspension, for intravenous use. Press release, Monroe Township, New Jersey, USA, April 4, 2016. 
[34] S.K. Bhargava and A.K. Srivastava. Interaction of ultrasound with matter. In S.K. Bhargava, editor, Principles and practice of ultrasonography, chapter 3, pages 10-15. Jaypee Brothers Medical Publishers (P) Ltd., New Delhi, India, $1^{\text {st }}$ edition, 2002.

[35] Siemens Aktiengesellschaft, Wittelsbacherplatz 2, 80333, Munich, Germany. https://www.siemens.com.

[36] W.A. Kalender. X-ray computed tomography. Physics in Medicine and Bio$\log y$, 51(13):R29-R43, 2006.

[37] H.K. Ha, P.B. Sachs, J.R. Haaga, and F. Abdul-Karim. CT-guided liver biopsy: an update. Clinical Imaging, 15(2):99-104, 1991.

[38] L. Thanos, A. Zormpala, G. Papaioannou, K. Malagari, E. Brountzos, and D. Kelekis. Safety and efficacy of percutaneous CT-guided liver biopsy using an 18-gauge automated needle. European Journal of Internal Medicine, 16(8):571-574, 2005.

[39] B.J. Park, J.H. Byun, Y.H. Jin, H.J. Won, Y.M. Shin, K.W. Kim, S.J. Park, and P.N. Kim. CT-guided radiofrequency ablation for hepatocellular carcinomas that were undetectable at US: therapeutic effectiveness and safety. Journal of Vascular and Interventional Radiology, 20(4):490-499, 2009.

[40] F. Laspas, E. Sotiropoulou, S. Mylona, A. Manataki, P. Tsagouli, I. Tsangaridou, and L. Thanos. Computed tomography-guided radiofrequency ablation of hepatocellular carcinoma: treatment efficacy and complications. Journal of Gastrointestinal and Liver Diseases, 18(3):323-328, 2009.

[41] I. Tsai, W. Tsai, M. Chen, G. Chang, W. Tzeng, S. Chan, and C.C. Chen. CT-guided core biopsy of lung lesions: a primer. American Journal of Roentgenology, 193(5):1228-1235, 2009.

[42] G. Belfiore, G. Moggio, E. Tedeschi, M. Greco, R. Cioffi, F. Cincotti, and R. Rossi. CT-guided radiofrequency ablation: a potential complementary therapy for patients with unresectable primary lung cancer - a preliminary report of 33 patients. American Journal of Roentgenology, 183(4):1003-1011, 2004.

[43] R. Lencioni, L. Crocetti, R. Cioni, R. Suh, D. Glenn, D. Regge, T. Helmberger, A. Gillams, A. Frilling, M. Ambrogi, C. Bartolozzi, and A. Mussi. Response to radiofrequency ablation of pulmonary tumours: a prospective, intention-to-treat, multicentre clinical trial (the RAPTURE study). The Lancet Oncology, 9(7):621-628, 2008.

[44] M.D. Beland, E.J. Wasser, W.W. Mayo-Smith, and D.E. Dupuy. Primary non-small cell lung cancer: review of frequency, location, and time of recurrence after radiofrequency ablation. Radiology, 254(1):301-307, 2010. 
[45] A. Gillams, Z. Khan, P. Osborn, and W. Lees. Survival after radiofrequency ablation in 122 patients with inoperable colorectal lung metastases. CardioVascular and Interventional Radiology, 36(3):724-730, 2013.

[46] M.A. Kliewer, D.H. Sheafor, E.K. Paulson, R.S. Helsper, B.S. Hertzberg, and R.C. Nelson. Percutaneous liver biopsy: a cost-benefit analysis comparing sonographic and CT guidance. American Journal of Roentgenology, 173(5):1199-1202, 1999.

[47] D.V. Sahani and S.P. Kalva. Imaging the liver. The Oncologist, 9(4):385-397, 2004.

[48] J.R. McCarley and M.C. Soulen. Percutaneous ablation of hepatic tumors. Seminars in Interventional Radiology, 27(3):255-260, 2010.

[49] C.H. Cha, F.T. Lee, J.M. Gurney, B.K. Markhardt, T.F. Warner, F. Kelcz, and D.M. Mahvi. CT versus sonography for monitoring radiofrequency ablation in a porcine liver. American Journal of Roentgenology, 175(3):705-711, 2000 .

[50] B. Daly and P.A. Templeton. Real-time CT fluoroscopy: evolution of an interventional tool. Radiology, 211(2):309-315, 1999.

[51] H.S. Hwang, M.J. Chung, J.W. Lee, S.W. Shin, and K.S. Lee. C-arm conebeam CT-guided percutaneous transthoracic lung biopsy: usefulness in evaluation of small pulmonary nodules. American Roentgen Ray Society, 195(6):W400-W407, 2010.

[52] J.W. Choi, C.M. Park, J.M. Goo, Y. Park, W. Sung, H. Lee, S.M. Lee, J.Y. Ko, and M. Shim. C-arm cone-beam CT-guided percutaneous transthoracic needle biopsy of small $(\leqslant 20 \mathrm{~mm})$ lung nodules: diagnostic accuracy and complications in 161 patients. American Journal of Roentgenology, 199(3):W322-W330, 2012.

[53] J.Y. Choo, C.M. Park, N.K. Lee, S.M. Lee, H. Lee, and J.M. Goo. Percutaneous transthoracic needle biopsy of small $(\leqslant 1 \mathrm{~cm})$ lung nodules under C-arm cone-beam CT virtual navigation guidance. European Radiology, 23(3):712-719, 2013.

[54] S.M. Lee, C.M. Park, K.H. Lee, Y.E. Bahn, J.I. Kim, and J.M. Goo. C-arm cone-beam CT-guided percutaneous transthoracic needle biopsy of lung nodules: clinical experience in 1108 patients. Radiology, 271(1):291-300, 2014.

[55] M. Abdel-Rehim, M. Ronot, A. Sibert, and V. Vilgrain. Assessment of liver ablation using cone beam computed tomography. World Journal of Gastroenterology, 21(2):517-524, 2015. 
[56] N. Abi-Jaoudeh, A.M. Venkatesan, W. van der Sterren, A. Radaelli, B. Carelsen, and B.J. Wood. Clinical experience with cone-beam CT navigation for tumor ablation. Journal of Vascular and Interventional Radiology, 26(2):214-219, 2015.

[57] M. Morimoto, K. Numata, M. Kondo, A. Nozaki, S. Hamaguchi, S. Takebayashi, and K. Tanaka. C-Arm Cone Beam CT for Hepatic Tumor Ablation Under Real-Time 3D Imaging. American Journal of Roentgenology, 194(5):W452-W454, 2010.

[58] R.L. Cazzato, J. Battistuzzi, V. Catena, R.F. Grasso, B.B. Zobel, E. Schena, $X$. Buy, and J. Palussiere. Cone-beam computed tomography (CBCT) versus CT in lung ablation procedure: which is faster? CardioVascular and Interventional Radiology, 38(5):1231-1236, 2015.

[59] R.C. Orth, M.J. Wallace, and M.D. Kuo. C-arm cone-beam CT: general principles and technical considerations for use in interventional radiology. Journal of Vascular and Interventional Radiology, 19(6):814-820, 2008.

[60] Y. Cheng, S. Tsai, Y. Cheng, J. Chen, J. Chai, and C.C. Chen. Percutaneous transthoracic lung biopsy: comparison between C-arm cone-beam CT and conventional CT guidance. Translational Oncology, 8(4):258-264, 2015.

[61] B.M. Dale, M.A. Brown, and R.C. Semelka. MRI: Basic principles and applications. $5^{\text {th }}$ edition, 2015.

[62] F. Fischbach, J. Bunke, M. Thormann, G. Gaffke, K. Jungnickel, J. Smink, and J. Ricke. MR-guided freehand biopsy of liver lesions with fast continuous imaging using a 1.0-T open MRI scanner: Experience in 50 patients. CardioVascular and Interventional Radiology, 34(1):188-192, 2011.

[63] E. Salomonowitz. MR imaging-guided biopsy and therapeutic intervention in a closed-configuration magnet: Single-center series of 361 punctures. American Journal of Roentgenology, 177(1):159-163, 2001.

[64] J. Kettenbach, W. Köstler, E. Rücklinger, B. Gustorff, M. Hüpfl, F. Wolf, K. Peer, M. Weigner, J. Lammer, W. Müller, and S.N. Goldberg. Percutaneous saline-enhanced radiofrequency ablation of unresectable hepatic tumors: Initial experience in 26 patients. American Journal of Roentgenology, 180(6):1537-1545, 2003.

[65] J. Kariniemi, R. Blanco Sequeiros, R. Ojala, and O. Tervonen. MRI-guided abdominal biopsy in a 0.23-T open-configuration MRI system. European Radiology, 15(6):1256-1262, 2005.

[66] J. Stattaus, S. Maderwald, H.A. Baba, G. Gerken, J. Barkhausen, M. Forsting, and M.E. Ladd. MR-guided liver biopsy within a short, wide-bore 1.5 Tesla MR system. European Radiology, 18(12):2865-2873, 2008. 
[67] M. Moche, S. Heinig, N. Garnov, J. Fuchs, T. Petersen, D. Seider, P. Brandmaier, T. Kahn, and H. Busse. Navigated MRI-guided liver biopsies in a closed-bore scanner: experience in 52 patients. European Radiology, 26(8):2462-2470, 2016.

[68] M. Liu, Y. Lv, L. Wu, J. Song, R.B. Sequeiros, and C. Li. MRI-guided percutaneous coaxial cutting needle biopsy of small pulmonary nodules: feasibility. European Radiology, 23(10):2730-2738, 2013.

[69] S. Clasen and P.L. Pereira. Magnetic resonance guidance for radiofrequency ablation of liver tumors. Journal of Magnetic Resonance Imaging, 27(2):421433, 2008.

[70] F.G. Abtin, J. Eradat, A.J. Gutierrez, C. Lee, M.C. Fishbein, and R.D. Suh. Radiofrequency ablation of lung tumors: imaging features of the postablation zone. RadioGraphics, 32(4):947-969, 2012.

[71] M. Arnolli, M. Franken, and M. Buijze, inventors. DEMCON Advanced Mechatronics B.V., applicant. System and method for aligning a medical device. WO patent application, WO2015/041516 A1, September 23, 2013.

[72] M.M. Arnolli, N.C. Hanumara, M. Franken, D.M. Brouwer, and I.A.M.J. Broeders. An overview of systems for CT- and MRI-guided percutaneous needle placement in the thorax and abdomen. The International Journal of Medical Robotics and Computer Assisted Surgery, 11(4):458-475, 2015.

[73] E.A. Spiegel, H.T. Wycis, M. Marks, and A.J. Lee. Stereotaxic apparatus for operations on the human brain. Science, 106(2754):349-350, 1947.

[74] Y.S. Kwoh, J. Hou, E.A. Jonckheere, and S. Hayati. A robot with improved absolute positioning accuracy for CT guided stereotactic brain surgery. IEEE Transactions on Biomedical Engineering, 35(2):153-160, 1988.

[75] G. Fichtinger, E.C. Burdette, A. Tanacs, A. Patriciu, D. Mazilu, L.L. Whitcomb, and D. Stoianovici. Robotically assisted prostate brachytherapy with transrectal ultrasound guidance - phantom experiments. Brachytherapy, 5(1):14-26, 2006.

[76] M. Muntener, A. Patriciu, D. Petrisor, D. Mazilu, H. Bagga, L. Kavoussi, K. Cleary, and D. Stoianovici. Magnetic resonance imaging compatible robotic system for fully automated brachytherapy seed placement. Urology, 68(6):1313-1317, 2006.

[77] R.H. Taylor and D. Stoianovici. Medical robotics in computer-integrated surgery. IEEE Transactions on Robotics and Automation, 19(5):765-781, 2003.

[78] K. Cleary, A. Melzer, V. Watson, G. Kronreif, and D. Stoianovici. Interventional robotic systems: applications and technology state-of-the-art. Minimally Invasive Therapy \& Allied Technologies, 15(2):101-113, 2006. 
[79] Stryker, 2825 Airview Boulevard, Kalamazoo, Michigan, 49002, USA. http://www.stryker.com.

[80] C. Wu, inventor; Stryker Corporation, assignee. Shielded surgical navigation system that determines the position and orientation of the tracked object with real and virtual dipoles. US patent 8,571,636 B2, October 29, 2013.

[81] ActiViews, Inc., 591 North Avenue, Entry One, Wakefield, Massachusetts, 01880, USA. http://www.activiews.com.

[82] P. Gilboa, inventor; Activiews Ltd., assignee. System and method for optical position measurement and guidance of a rigid or semi-flexible tool to a target. US patent 7,876,942 B2, January 25, 2011.

[83] Northern Digital Inc., 103 Randall Drive, Waterloo, Ontario, N2V 1C5, Canada. http:/ / www.ndigital.com.

[84] E.B. Levy, J. Tang, D. Lindisch, N. Glossop, F. Banovac, and K. Cleary. Implementation of an electromagnetic tracking system for accurate intrahepatic puncture needle guidance: accuracy results in an in vitro model. Academic Radiology, 14(3):344-354, 2007.

[85] Royal Philips, Amstelplein 2, Breitner Center, 1096 BC, Amsterdam, the Netherlands. http://www.philips.com.

[86] J.H. Yanof, L.L. Goldstein, F.C. Jensen, J.C. Foster, and C. Bauer, inventors; Picker International Inc., assignee. Interchangeable guidance devices for C.T. assisted surgery and method of using same. US patent $5,957,933$, September 28, 1999.

[87] Claron Technology Inc., 120 Carlton Street, Suite 217, Toronto, Ontario, M5A 4K2, Canada. http:/ / www.clarontech.com.

[88] L. Maier-Hein, A. Tekbas, A. Seitel, F. Pianka, S.A. Müller, S. Satzl, S. Schawo, B. Radeleff, R. Tetzlaff, A.M. Franz, B.P. Müller-Stich, I. Wolf, H. Kauczor, B.M. Schmied, and H. Meinzer. In vivo accuracy assessment of a needle-based navigation system for CT-guided radiofrequency ablation of the liver. Medical Physics, 35(12):5385-5396, 2008.

[89] K.A. Gavaghan, S. Anderegg, M. Peterhans, T. Oliveira-Santos, and S. Weber. Augmented reality image overlay projection for image guided open liver ablation of metastatic liver cancer. In Augmented Environments for Computer-Assisted Interventions, volume 7264 of Lecture Notes in Computer Science, pages 36-46. 2012.

[90] Medtronic, 710 Medtronic Parkway, Minneapolis, Minnesota, 55432-5604, USA. http://www.medtronic.com. 
[91] Pathfinder, 2969 Armory Drive, Suite 100A, Nashville, Tennessee, 37204, USA. http:/ / www.pathnav.com.

[92] CAScination AG, Stauffacherstrasse 78, 3008 Bern, Switserland. http:/ / www.cascination.com.

[93] F. Banovac, N. Glossop, D. Lindisch, D. Tanaka, E. Levy, and K. Cleary. Liver tumor biopsy in a respiring phantom with the assistance of a novel electromagnetic navigation device. In Proceedings of the International Conference on Medical Image Computing and Computer-Assisted Intervention (MICCAI), volume 2488 of Lecture Notes in Computer Science, pages 200-207, 2002.

[94] B.J. Wood, H. Zhang, A. Durrani, N. Glossop, S. Ranjan, D. Lindisch, E. Levy, F. Banovac, J. Borgert, S. Krueger, J. Kruecker, A. Viswanathan, and K. Cleary. Navigation with electromagnetic tracking for interventional radiology procedures: a feasibility study. Journal of Vascular and Interventional Radiology, 16(4):493-505, 2005.

[95] M. Nagel, M. Hoheisel, R. Petzold, W.A. Kalender, and U.H.W. Krause. Needle and catheter navigation using electromagnetic tracking for computer-assisted C-arm CT interventions. In Medical Imaging 2007: Visualization and Image-Guided Procedures. Proceedings of the SPIE, volume 6509, pages 65090J-1-65090J-9, 2007.

[96] Krücker, J. and Xu, S. and Glossop, N. and Viswanathan, A. and Borgert, J. and Schulz, H. and Wood, B.J. Electromagnetic tracking for thermal ablation and biopsy guidance: clinical evaluation of spatial accuracy. Journal of Vascular and Interventional Radiology, 18(9):1141-1150, 2007.

[97] B.C. Meyer, O. Peter, M. Nagel, M. Hoheisel, B.B. Frericks, K. Wolf, and F.K. Wacker. Electromagnetic field-based navigation for percutaneous punctures on C-arm CT: experimental evaluation and clinical application. European Radiology, 18(12):2855-2864, 2008.

[98] M. Nagel, M. Hoheisel, U. Bill, K. Klingenbeck-Regn, W.A. Kalender, and R. Petzold. Electromagnetic tracking system for minimal invasive interventions using a C-arm system with CT option: first clinical results. In Medical Imaging 2008: Visualization, Image-guided Procedures, and Modeling. Proceedings of the SPIE, volume 6918, pages 69180G-1-69180G-10, 2008.

[99] J.H. Yanof, P.H. Klahr, and L. O’Donnell, inventors; Picker International Inc., assignee. Frameless stereotactic CT scanner with virtual needle display for planning image guided interventional procedures. US patent 6,064,904, May 16, 2000.

[100] J.M. Hevezi, M. Blough, D. Hoffmeyer, and J.H. Yanof. Brachytherapy using CT PinPoint. MEDICAMUNDI, 46(3):22-27, 2002. 
[101] Immersion Corporation, 30 Rio Robles, San Jose, California, 95134, USA. http:/ /www.immersion.com.

[102] A.M. Palestrant, inventor. Guidance device for C.T. guided drainage and biopsy procedures. US patent 4,733,661, March 29, 1988.

[103] A. Palestrant, inventor. Guidance device for C.T. guided drainage and biopsy procedures. US patent 5,102,391, April 7, 1992.

[104] H. Zhang, S. Feng, and W. Feng, inventors; H. Zhang, S. Feng, and Zhejiang Kindly Medical Devices Co., Ltd., assignees. Guided puncturing needle and puncturing guiding method. US patent 8,241,301 B2, August 14, 2012.

[105] Inrad, Inc., 4375 Donker Ct. SE, Kentwood, Michigan, 49512, USA. http://www.inrad-inc.com.

[106] R.E. Davis, and J.E. Drace, inventors; DLP, Inc., assignee. Goniometer for needle placement. US patent 5,196,019, March 23, 1993.

[107] A.M. Palestrant. Comprehensive approach to CT-guided procedures with a hand-held guidance device. Radiology, 174(1):270-272, 1990.

[108] P.R. Frederick, inventor. Three-dimensional light guidance system for invasive procedures. US patent 4,651,732, March 24, 1987.

[109] E. Unger, and F.S. Pereles, inventors; ImaRx Pharmaceutical Corp., assignee. Apparatus for performing biopsies and the like. US patent 5,628,327, May 13, 1997.

[110] LAP GmbH Laser Applikationen, Zeppelinstraße 23, 21337, Lüneburg, Germany. http:/ / www.lap-laser.com.

[111] V. Jacobi, A. Thalhammer, and J. Kirchner. Value of a laser guidance system for CT interventions: a phantom study. European Radiology, 9(1):137-140, 1999.

[112] NeoRad AS, Gaustadalléen 21, 0349, Oslo, Norway. http://www.neorad.no.

[113] K. Brabrand, inventor; NeoRad A/S, assignee. Apparatus for assisting percutaneous computed tomography-guided surgical activity. US patent 6,021,342, February 1, 2000.

[114] amedo Smart Tracking Solutions GmbH, Universitätsstraße 142, 44799, Bochum, Germany. http://www.amedo-gmbh.com. 
[115] C. Moser, J. Becker, M. Deli, M. Busch, M. Boehme, and D.H.W. Groenemeyer. A novel laser navigation system reduces radiation exposure and improves accuracy and workflow of CT-guided spinal interventions: a prospective, randomized, controlled, clinical trial in comparison to conventional freehand puncture. European Journal of Radiology, 82(4):627-632, 2013.

[116] P.R. Frederick, T.H. Brown, M.H. Miller, A.L. Bahr, and K.H. Taylor. A lightguidance system to be used for CT-guided biopsy. Radiology, 154(2):535536, 1985.

[117] H. Ishizaka, T. Katsuya, Y. Koyama, H. Ishijima, T. Moteki, and J. Aoki. CT-guided percutaneous intervention using a simple laser director device. American Journal of Roentgenology, 170(3):745-746, 1998.

[118] F.S. Pereles, M. Baker, R. Baldwin, E. Krupinski, and E.C. Unger. Accuracy of CT biopsy: laser guidance versus conventional freehand techniques. Academic Radiology, 5(11):766-770, 1998.

[119] A. Rockseisen, inventor; LAP GmbH Laser Applikationen, assignee. Target apparatus for linearly inserting an instrument into a human body. US patent 5,707,360, January 13, 1998.

[120] A. Rockseisen, inventor; LAP GmbH, assignee. Targetting device for the straight-lined introduction of an instrument into a human body. US patent 6,044,291, March 28, 2000.

[121] R. Kloeppel, T. Weisse, F. Deckert, W. Wilke, and S. Pecher. CT-guided intervention using a patient laser marker system. European Radiology, 10(6):1010-1014, 2000.

[122] J. Regn, inventor; Siemens Aktiengesellschaft, assignee. Device for making a guide path for an instrument on a patient. US patent 6,041,249, March 21, 2000.

[123] K. Brabrand, inventor; NeoRad A/S, assignee. Method and apparatus for assisting percutaneous computed tomography-guided surgical activity. US patent 6,334,067 B1, December 25, 2001.

[124] K. Brabrand, O.H. Eriksen, Ø. Låg, J.M. Bendiksen, D.A. Hansen, and S. Småstuen, inventors; NeoRad A/S, assignee. Apparatus for light beam guided biopsy. US patent 6,443,960 B1, September 3, 2002.

[125] G.A. Krombach, T. Schmitz-Rode, B.B. Wein, J. Meyer, J.E. Wildberger, K. Brabant, and R.W. Günther. Potential of a new laser target system for percutaneous CT-guided nerve blocks: technical note. Neuroradiology, 42(11):838-841, 2000. 
[126] Z. Varro, J.K. Locklin, and B.J. Wood. Laser navigation for radiofrequency ablation. Cardiovascular and Interventional Radiology, 27(5):512-515, 2004.

[127] M. Busch, M. Deli, J. Speder, and D. Groenemeyer, inventors; amedo $\mathrm{GmbH}$, assignee. Needle positioning system. World Intellectual Property Organization patent WO 2006/125605 A1, November 30, 2006.

[128] B.J. Wood and J.H. Yanof. CT-guided interventional oncology - bridging the gap between diagnosis and therapy. MEDICAMUNDI, 49(3):28-32, 2005.

[129] J. Yanof, C. Bauer, S. Renisch, A. Lala, P. Klahr, D. Haemmerich, and B. Wood. CT-integrated treatment planning for robot-assisted, laser-guided radiofrequency ablation. In Proceedings of the Computer Assisted Radiology and Surgery 19th International Congress and Exhibition, volume 1281, page 1361, 2005.

[130] M.Z. Chang, C.M. Yeh, and C.Y. Yeh, inventors. Positioning device for percutaneous needle insertion. US patent 4,841,967, June 27, 1989.

[131] K. Brabrand, N. Bérard-Andersen, G.F. Olsen, and V. Bakke, inventors; NeoRad A/S, applicant. Needle holder. US patent application 2012/0022368 A1, January 26, 2012.

[132] AprioMed AB, Virdings Allé 28, 754 50, Uppsala, Sweden. http://www.apriomed.com.

[133] A. Eklund, A. Tiensuu, and E. Nicklasson, inventors; RADI MEDICAL SYSTEMS AB, applicant. Medical guide for guiding a medical instrument. US patent application 2008/0200798 A1, August 21, 2008.

[134] R. Gupta, S.R.H. Barrett, N.C. Hanumara, J.O. Shepard, A.H. Slocum, and C.J. Walsh, inventors. Guidance and insertion system. US patent application 2006/0229641 A1, October 12, 2006.

[135] S. Song, J. Tokuda, K. Tuncali, A. Yamada, M. Torabi, and N. Hata. Design evaluation of a double ring RCM mechanism for robotic needle guidance in MRI-guided liver interventions. In Proceedings of the IEEE/RSJ International Conference on Intelligent Robots and Systems (IROS), pages 4078-4083, 2013.

[136] M. Demathelin, and B. Maurin, B. Bayle, J. Gangloff, and O. Piccin, inventors; Institut National des Sciences Appliquees, Institut de Recherche sur les Cancers de l'Appareil Digestif - IRCAD, Universite Louis Pasteur, and Centre National de la Recherche Scientifique, assignees. Robotic positioning and orientation device and needle holder comprising one such device. US patent 7,881,823 B2, February 1, 2011.

[137] I. Bricault, N. Zemiti, E. Jouniaux, C. Fouard, E. Taillant, F. Dorandeu, and P. Cinquin. A Light Puncture Robot for CT and MRI interventions. IEEE Engineering in Medicine and Biology Magazine, 27(3):42-50, 2008. 
[138] A. Magnusson, E. Radecka, M. Lönnemark, and H. Raland. Computedtomography-guided punctures using a new guidance device. Acta Radiologica, 46(5):505-509, 2005.

[139] A. Magnusson, and P. Egnelöv, inventors; Aprio Medical AB, assignee. Guide for a medical device. US patent 7,824,417 B2, November 2, 2010.

[140] S.R.H. Barrett, N.C. Hanumara, C.J. Walsh, A.H. Slocum, R. Gupta, and J.O. Shepard. A remote needle guidance system for percutaneous biopsies. In Proceedings of the ASME 2005 International Design Engineering Technical Conferences and Computers and Information in Engineering Conference (IDETC/CIE), volume 7, pages 481-189, Long Beach, California, USA, 2005.

[141] C.J. Walsh, N.C. Hanumara, A.H. Slocum, J. Shepard, and R. Gupta. A patient-mounted, telerobotic tool for CT-guided percutaneous interventions. Journal of Medical Devices, 2(1):011007-1-011007-10, 2008.

[142] F.Y. Wu, M. Torabi, A. Yamada, A. Golden, G.S. Fischer, K. Tuncali, D.D. Frey, and C. Walsh. An MRI coil-mounted multi-probe robotic positioner for cryoablation. In Proceedings of the ASME 2013 International Design Engineering Technical Conferences and Computers and Information in Engineering Conference (IDETC/CIE), volume 6A, Portland, Oregon, USA, 2013.

[143] B. Maurin, O. Piccin, B. Bayle, J. Gangloff, M. de Mathelin, L. Soler, and A. Gangi. A new robotic system for CT-guided percutaneous procedures with haptic feedback. In Proceedings of the Computer Assisted Radiology and Surgery 18th International Congress and Exhibition (CARS), volume 1268, pages 515-520, Chicago, Illinois, USA, 2004.

[144] B. Maurin, O. Piccin, B. Bayle, J. Gangloff, and M. de Mathelin. A parallel 5 DOF positioner for semi-spherical workspaces. In Proceedings of the ASME 2004 International Design Engineering Technical Conferences and Computers and Information in Engineering Conference (IDETC/CIE), volume 2, pages 10331042, Salt Lake City, Utah, USA, 2004.

[145] E. Taillant, J. Avila-Vilchis, C. Allegrini, I. Bricault, and P. Cinquin. CT and MR compatible Light Puncture Robot: architectural design and first experiments. In Proceedings of the International Conference on Medical Image Computing and Computer-Assisted Intervention (MICCAI), volume 3217 of Lecture Notes in Computer Science, pages 145-152. 2004.

[146] N. Hungr, C. Fouard, A. Robert, I. Bricault, and P. Cinquin. Interventional radiology robot for $\mathrm{CT}$ and MRI guided percutaneous interventions. In Proceedings of the International Conference on Medical Image Computing and Computer-Assisted Intervention (MICCAI), volume 6891 of Part I, Lecture Notes in Computer Science, pages 137-144, 2011. 
[147] Bard International, Inc., 730 Central Avenue, Murray Hill, New Jersey, 07974, USA. http:/ / www.crbard.com.

[148] A. Magnusson, and D. Åkerfeldt, inventors; Bard International, Inc., assignee. Puncture guide for computer tomography. US patent 5,280,427, January 18, 1994.

[149] E.G. Christoforou, I. Seimenis, E. Andreou, E. Eracleous, and N.V. Tsekos. A novel, general-purpose, MR-compatible, manually actuated robotic manipulation system for minimally invasive interventions under direct MRI guidance. The International Journal of Medical Robotics and Computer Assisted Surgery, 10(1):22-34, 2014.

[150] M. Loser, inventor; Siemens Aktiengesellschaft, assignee. Medical device with a drive unit for a needle. US patent 7,175,635 B2, February 13, 2007.

[151] iSYS Medizintechnik GmbH, Bergwerksweg 21, 6370, Kitzbühel, Austria. http:/ / www.isys.co.at.

[152] M. Vogele, inventor. Device for controlling corporeal structures. US patent application 2007/0276407 A1, November 29, 2007.

[153] D. Stoianovici, D. Mazilu, and L.R. Kavoussi, inventors; The Johns Hopkins University, assignee. Robot for computed tomography interventions. US patent 7,822,466 B2, October 26, 2010.

[154] Innomedic GmBh, Technologiezentrum Herxheim, Luitpoldstrae 59, 76863, Herxheim, Germany. http://www.innomedic.de.

[155] A. Melzer, B. Gutmann, T. Remmele, R. Wolf, A. Lukoscheck, M. Bock, $\mathrm{H}$. Bardenheuer, and $\mathrm{H}$. Fischer. INNOMOTION for percutaneous imageguided interventions - principles and evaluation of this MR- and CTcompatible robotic system. IEEE Engineering in Medicine and Biology Magazine, 27(3):66-73, 2008.

[156] A. Magnusson and D. Åkerfeldt. CT-guided core biopsy using a new guidance device. Acta Radiologica, 32(1):83-85, 1991.

[157] K.T. Brown, G.I. Getrajdman, and J.F. Botet. Clinical trial of the Bard CT guide system. Journal of Vascular and Interventional Radiology, 6(3):405-10, 1995.

[158] M.H. Loser and N. Navab. A new robotic system for visually controlled percutaneous interventions under CT fluoroscopy. In Proceedings of the International Conference on Medical Image Computing and Computer-Assisted Intervention (MICCAI), volume 1935 of Lecture Notes in Computer Science, pages 887-896, 2000. 
[159] D. Stoianovici, K. Cleary, A. Patriciu, D. Mazilu, A. Stanimir, N. Craciunoiu, V. Watson, and L. Kavoussi. AcuBot: a robot for radiological interventions. IEEE Transactions on Robotics and Automation, 19(5):927-930, 2003.

[160] S. Shah, A. Kapoor, J. Ding, P. Guion, D. Petrisor, J. Karanian, W.F. Pritchard, D. Stoianovici, B.J. Wood, and K. Cleary. Robotically assisted needle driver: evaluation of safety release, force profiles, and needle spin in a swine abdominal model. International Journal of Computer Assisted Radiology and Surgery, 3(1-2):173-179, 2008.

[161] R. Susil, J. Anderson, and R. Taylor. A single image registration method for CT guided interventions. In Proceedings of the International Conference on Medical Image Computing and Computer-Assisted Intervention (MICCAI), Lecture Notes in Computer Science, pages 798-808, 1999.

[162] A. Melzer, B. Gutmann, A. Lukoscheck, M. Mark, W. Zylka, and H. Fischer. Experimental evaluation of an MR compatible telerobotic system for CT MR-guided interventions. Supplement to Radiology, 226(409):\#444, 2003.

[163] Synthes GmbH, Luzernstrasse 21, 4528, Zuchwil, Switzerland. http:/ / www.synthes.com.

[164] G.M. Onik, E.R.Cosman, and T.H. Wells, inventors. Method and apparatus for stereotaxic placement of probes in the body utilizing CT scanner localization. US patent 4,583,538, April 22, 1986.

[165] P.G. Koutrouvelis, inventor. Stereotactic device. US patent 5,047,036, September 10, 1991.

[166] C.D. Bidwell, inventor. Biopsy needle guide for use with CT scanner. US patent 5,053,042, October 1, 1991.

[167] N. Hata, J. Tokuda, S. Hurwitz, and S. Morikawa. MRI-compatible manipulator with remote-center-of-motion control. Journal of Magnetic Resonance Imaging, 27(5):1130-1138, 2008.

[168] K. Chinzei, N. Hata, F. Jolesz, and R. Kikinis. MR compatible surgical assist robot: system integration and preliminary feasibility study. In Proceedings of the International Conference on Medical Image Computing and ComputerAssisted Intervention (MICCAI), volume 1935 of Lecture Notes in Computer Science, pages 921-930. 2000.

[169] Perfint Healthcare Pvt. Ltd., 3rd Floor, No. 16, Southwest Boag Road, T. Nagar, Chennai, Tamil Nadu 600017, India. http:/ / www.perfinthealthcare.com.

[170] G.K Velusamy, inventor, Perfint Healthcare Private Limited, applicant. 2013. European patent application 2,561,821 A1, February 27, Tool positioning system. 
[171] J.H. Yanof, K.J. West, C. Bauer, D.M. Kwartowitz, inventors; Koninklijke Philips Electronics, N.V., assignee. Tactile feedback and display in a CT image guided robotic system for interventional procedures. US patent 6,785,572 B2, August 31, 2004.

[172] Y. Zhou, K. Thiruvalluvan, L. Krzeminski, W.H. Moore, Z. Xu, and Z. Liang. CT-guided robotic needle biopsy of lung nodules with respiratory motion Experimental system and preliminary test. The International Journal of Medical Robotics and Computer Assisted Surgery, 9(3):317-330, 2013.

[173] S. Tovar-Arriaga, R. Tita, J.C. Pedraza-Ortega, E. Gorrostieta, and W.A. Kalender. Development of a robotic FD-CT-guided navigation system for needle placement - preliminary accuracy tests. The International Journal of Medical Robotics and Computer Assisted Surgery, 7(2):225-236, 2011.

[174] G. Onik, P. Costello, E. Cosman, T. Wells, Jr., H. Goldberg, A. Moss, R. Kane, M.E. Clouse, W. Hoddick, S. Moore, and B. Demas. CT body stereotaxis: an aid for CT-guided biopsies. American Journal of Roentgenology, 146(1):163$168,1986$.

[175] S. Morikawa, S. Naka, K. Murakami, Y. Kurumi, H. Shiomi, T. Tani, H.A. Haque, J. Tokuda, N. Hata, and T. Inubushi. Preliminary clinical experiences of a motorized manipulator for magnetic resonance image-guided microwave coagulation therapy of liver tumors. The American Journal of Surgery, 198(3):340-347, 2009.

[176] J. Yanof, J. Haaga, P. Klahr, C. Bauer, D. Nakamoto, A. Chaturvedi, and R. Bruce. CT-integrated robot for interventional procedures: preliminary experiment and computer-human interfaces. Computer Aided Surgery, 6(6):352-359, 2001.

[177] J. Yanof, C. Bauer, and B. Wood. Tactile feedback and display system for CT-guided, robot-assisted percutaneous procedures. In Proceedings of the Computer Assisted Radiology and Surgery 18th International Congress and Exhibition (CARS), volume 1268, pages 521-526, Chicago, Illinois, USA, 2004.

[178] Y. Zhou, K. Thiruvalluvan, L. Krzeminski, W.H. Moore, Z. Xu, and Z. Liang. An experimental system for robotic needle biopsy of lung nodules with respiratory motion. In Proceedings of the 2011 IEEE International Conference on Mechatronics and Automation, pages 823-830, Beijing, China, 2011.

[179] S. Tovar-Arriaga, J.E. Vargas, J.M. Ramos, M.A. Aceves, E. Gorrostieta, and W.A. Kalender. A fully sensorized cooperative robotic system for surgical interventions. Sensors, 12(7):9423-9447, 2012.

[180] C.M. Chen, J.W.C. Chang, Y.C. Cheung, G. Lin, J.J. Hsieh, T. Hsu, and S.F. Huang. Computed tomography-guided core-needle biopsy specimens 
demonstrate epidermal growth factor receptor mutations in patients with non-small-cell lung cancer. Acta Radiologica, 49(9):991-994, 2008.

[181] M.M. Arnolli, M. Franken, and D.M. Brouwer. CT registration: experimental determination of suited fiducial marker material and registration errors. Journal of Medical Devices, 7:020946-1-020946-2, 2013.

[182] Maxon Motor AG, Brünigstrasse 220, 6072, Sachseln, Switzerland. http://www.maxonmotor.com.

[183] J.F. Barrett and N. Keat. Artifacts in CT: recognition and avoidance. RadioGraphics, 24(6):1679-1691, 2004.

[184] G.B. Drummond and D.H.T. Scott. Deflection of spinal needles by the bevel. Anaesthesia, 35(9):854-857, 1980.

[185] R.K. Baumgarten. Importance of the needle bevel during spinal and epidural anesthesia. Regional Anaesthesia, 20(3):234-238, 1995.

[186] B.T. Sitzman and D.R. Uncles. The effects of needle type, gauge, and tip bend on spinal needle deflection. Regional Anaesthesia and Pain Management, 82(2):297-301, 1996.

[187] M.N. Hochmann and M.J. Friedman. In vitro study of needle deflection: a linear insertion technique versus a bidirectional rotation insertion technique. Quintessence International, 31(1):33-39, 2000.

[188] N. Abolhassani and R.V. Patel. Deflection of a flexible needle during insertion into soft tissue. In Proceedings of the 28th IEEE Engineering in Medicine and Biology Society Annual International Conference, pages 3858-3861, 2006.

[189] N. Abolhassani and R.V. Patel. Minimization of needle deflection in robotassisted prostate brachytherapy. In International Journal of Computer Assisted Radiology and Surgery, volume 1, pages 269-271, 2006.

[190] N. Abolhassani, R. Patel, and F. Ayazi. Effects of different insertion methods on reducing needle deflection. In Proceedings of the 29th IEEE Engineering in Medicine and Biology Society Annual International Conference, pages 491-494, 2007.

[191] N. Abolhassani, R.V. Patel, and F. Ayazi. Minimization of needle deflection in robot-assisted percutaneous therapy. The International Journal of Medical Robotics and Computer Assisted Surgery, 3(2):140-148, 2007.

[192] S. Misra, K.B. Reed, B.W. Schafer, K. T. Ramesh, and A.M. Okamura. Observations and models for needle-tissue interactions. In IEEE International Conference on Robotics and Automation, pages 2687-2692, Kobe, Japan, 2009. 
[193] S. Misra, K.B. Reed, B.W. Schafer, K.T. Ramesh, and A.M. Okamura. Mechanics of flexible needles robotically steered through soft tissue. The International Journal of Robotics Research, 29(13):1640-1660, 2010.

[194] A. Jahya, F. van der Heijden, and S. Misra. Observations of threedimensional needle deflection during insertion into soft tissue. In Proceedings of the 4th IEEE RAS/EMBS International Conference on Biomedical Robotics and Biomechatronics, pages 1205-1210, Rome, Italy, 2012.

[195] R.J. Roesthuis, Y.R.J. van Veen, A. Jahya, and S. Misra. Mechanics of needletissue interaction. In Proceedings of the IEEE/RSJ International Conference on Intelligent Robots and Systems, pages 2557-2563, 2011.

[196] Y.R.J. van Veen, A. Jahya, and S. Misra. Macroscopic and microscopic observations of needle insertion into gels. In Proceedings of the Institution of Mechanical Engineers, Part H: Journal of Engineering in Medicine, pages 441449, 2012.

[197] H. Kataoka, T. Washio, M.A. Audette, and K. Mizuhara. A model for relations between needle deflection, force, and thickness on needle penetration. In Medical Image Computing and Computer-Assisted Intervention, volume 2208 of Lecture Notes in Computer Science, pages 966-974, 2001.

[198] S.P. DiMaio and S.E. Salcudean. Needle insertion modeling and simulation. IEEE Transactions on Robotics and Automation, 19(5):864-875, 2003.

[199] A. Majewicz, S.P. Marra, M.G. van Vledder, M. Lin, M.A. Choti, D.Y. Song, and A.M. Okamura. Behavior of tip-steerable needles in ex vivo and in vivo tissue. IEEE Transactions on Biomedical Engineering, 59(10):2705-2715, 2012.

[200] R.J. Webster, J.S. Kim, N.J. Cowan, G.S. Chirikjian, and A.M. Okamura. Nonholonomic modeling of needle steering. The International Journal of Robotics Research, 25(5-6):509-525, 2006.

[201] J. Ding, D. Stoianovici, D. Petrisor, P. Mozer, R. Avila, L. Ibanez, W. Turner, D. Yankelvitz, E. Wilson, F. Banovac, and K. Cleary. Medical needle steering for lung biopsy: experimental results in tissue phantoms using a robotic needle driver. In Proceedings of the 8th IEEE International Conference on Bioinformatics and Bioengineering, pages 1-5, 2008.

[202] K.B. Reed, A. Majewicz, V. Kallem, R. Alterovitz, K. Goldberg, N.J. Cowan, and A.M. Okamura. Robot-assisted needle steering. IEEE Robotics Automation Magazine, 18(4):35-46, 2011.

[203] Medax, Via S. Pertini, 4, 41039, San Possidonio, Italy. http://medax.org/.

[204] Becton, Dickinson and Company - Carefusion, 1 Becton Drive, Franklin Lakes, New York, 07417, USA. http:/ / www.carefusion.com. 
[205] AngioDynamics, 14 Plaza Drive, Latham, New York, 12110, USA. http:/ / www.angiodynamics.com.

[206] Medtronic - Covidien, 710 Medtronic Parkway, Minneapolis, 55432, USA. http://www.medtronic.com/covidien.

[207] Boston Scientific, 300 Boston Scientific Way, Marlborough, Massachusetts, 01752, USA. http:/ / www.bostonscientific.com.

[208] RF Medical Co., Ltd., 503 World Meridian, 60-24 Gasan-dong, Geumcheongu, Seoul, South Korea. http:/ / www.rfa.co.kr.

[209] The MathWorks Inc., 1 Apple Hill Drive, Natick, Massachusetts, 01760, USA. http:/ / www.mathworks.com.

[210] Arduino. https://www.arduino.cc.

[211] P. Sprawls, Jr. Physical principles of medical imaging. Medical Physics Publishing, Madison, Wisconsin, USA, second edition, 1995.

[212] FARO, 250 Technology Park, Lake Mary, Florida, 32746, USA. http://www.faro.com.

[213] Elmo Motion Control Ltd., 60 Amal Street, Petach-Tikva, 4951360, Israel. http:/ / www.elmomc.com.

[214] INDUcoder Messtechnik GmbH, Kaiserstraße 316, 47178, Duisburg, Germany. http:/ / www.inducoder.de.

[215] Käfer Messuhrenfabrik GmbH \& Co. KG, Hahnstraße 11, 78054, VillingenSchwenningen, Germany. http://www.kaefer-messuhren.de.

[216] T. Gruber-Rouh, C. Lee, J. Bolck, N.N.N. Naguib, B. Schulz, K. Eichler, R. Aschenbach, J.L Wichmann, T.J Vogl, and S. Zangos. Intervention planning using a laser navigation system for CT-guided interventions: a phantom and patient study. Korean journal of radiology, 16(4):729_735, 2015.

[217] D. Wallach, G. Toporek, S. Weber, R. Bale, and G. Widmann. Comparison of freehand-navigated and aiming device-navigated targeting of liver lesions. The International Journal of Medical Robotics and Computer Assisted Surgery, 10(1):35-43, 2014.

[218] Y. Koethe, S. Xu, G. Velusamy, B.J. Wood, and A.M. Venkatesan. Accuracy and efficacy of percutaneous biopsy and ablation using robotic assistance under computed tomography guidance: a phantom study. European Radiology, 24(3):723-730, 2014. 
[219] J. Kettenbach, L. Kara, G. Toporek, M. Fuerst, and G. Kronreif. A robotic needle-positioning and guidance system for CT-guided puncture: ex vivo results. Minimally Invasive Therapy \& Allied Technologies, 23(5):271-278, 2014.

[220] IMAGE Information Systems Ltd., $3^{\text {rd }}$ floor, 207 Regent Street, London, W1B 3HH, United Kingdom. http:/ / www.k-pacs.net.

[221] European Commission. European guidelines on quality criteria for Computed Tomography. 2000.

[222] International Business Machines Corp., 1 New Orchard Road, Armonk, New York, 10504-1722, USA. http://www.ibm.com.

[223] Philips Healthcare, Veenpluis 4-6, 5684 PC, Best, the Netherlands. http:/ / www.philips.nl/healthcare.

[224] Fraunhofer Project group for Automation in Medicine and Biotechnology (PAMB), Theodor-Kutzer-Ufer 1-3. Haus 8, Flur 1, 68167, Mannheim, Germany. http:/ / pamb.ipa.fraunhofer.de.

[225] G. Schaefers and A. Melzer. Testing methods for MR safety and compatibility of medical devices. Minimally Invasive Therapy $\mathcal{E}$ Allied Technologies, 15(2):71-75, 2006.

[226] G. Schaefers. Testing MR safety and compatibility. IEEE Engineering in Medicine and Biology Magazine, 27(3):23-27, May 2008.

[227] S.R. Majumdar. Pneumatic Systems: Principles and Maintenance. McGrawHill, 1996.

[228] P. Beater. Pneumatic Drives. Springer-Verlag Berlin Heidelberg, first edition, 2007.

[229] DEPRAG. No Metal: Amazing air motors made from ceramic materials. Press release, November, 2009. http://www.deprag.com/en/company/pressrelease/presse200911a.html.

[230] S. R. Pandian, F. Takemura, Y. Hayakawa, and S. Kawamura. Control performance of an air motor - can air motors replace electric motors? In Proceedings 1999 IEEE International Conference on Robotics and Automation (ICRA), volume 1, pages 518-524, Detroit, Michigan, USA, 1999.

[231] University Medical Center Utrecht, Heidelberglaan 100, 3584 CX, Utrecht, the Netherlands. http://www.umcutrecht.nl.

[232] Quirem Medical B.V., Zutphenseweg 55, 7418 AH, Deventer, the Netherlands. http://www.quirem.com. 
[233] University Medical Center Groningen, Hanzeplein 1, 9713 GZ, Groningen, the Netherlands. http:/ / www.umcg.nl.

[234] Health Care Inspectorate, Ministry of Health, Welfare and Sport, Postbus 2518, 6401 DA, Heerlen, the Netherlands. https:/ / www.igz.nl.

[235] DEMCON. DEMCON lanceert eigen merk voor medische robotica. Press release, June 30, 2016. https: / / www.demcon.nl/demcon-medical-roboticsnieuwe-loot-aan-demcon-boom. 


\section{Acknowledgements}

Six years have passed since DEMCON granted me the privilege to conduct a postgraduate research in collaboration with the University of Twente on imageguided minimally invasive interventions, which I took as an opportunity to shape a vision on the design of medical devices and embed it in the presented system for CT-guided percutaneous needle placement. I would like to express my sincere gratitude to the following people who have been involved in the research. First of all, to Michel Franken (DEMCON) for joining the project as my direct supervisor, as a sparring partner in shaping the strategy and architecture of the novel system, and for his help in subdividing the research into separate publications and reviewing their individual contents. To Dannis Brouwer (University of Twente) for his involvement from the very beginning of the research as my academic supervisor with a focus on the technical realm of the research, for his input on the system's strategy and architecture related to the design principles for precision mechanisms. To Ivo Broeders (University of Twente) as my senior academic supervisor with a focus on the clinical realm of the research, for his review of the system's strategy and usability as an experienced clinical user of medical robotics, and guidance in the experiment design of the phantom study. To Michiel Jannink (DEMCON) as the initiator of the project, for entrusting it to me and for emphasizing the importance of a workflow based approach for the design of medical devices. To Nevan Hanumara (Massachusetts Institute of Technology) for his collaboration on the review of the state of the art of systems for CT- and MRI-guided needle placement in the thorax and abdomen and discussing their flaws. To Koert de Jong (University Medical Center Groningen) as a clinical user for illustrating the conventional freehand method of CT-guided percutaneous needle placement for liver tumour ablation in clinical practise and collaboration on the clinical deployment of the novel system.

I am deeply indebted to my colleagues in the multidisciplinary development team at DEMCON, including mechatronic system engineering, mechanical and industrial design engineering, electrical and electronic system engineering, software engineering, and prototyping, for their professionalism, dedication and perseverance to complete technical challenges on the verge of the impossible, even when hassled by impatience and ignorant underestimation. I would like to pay tribute to all who contributed over the course of the project, and to the following people in particular. To Vincent Heijmer for the project management and for support in practical decision making. To Michael Harmsen for the design of the electrical subsystems, his endurance to install the electrical wiring in the locking module arm, and help in filling the hydraulic system. To Frank Vlaardingerbroek for revising the GUI software to match the desired layout and interface with the registration, kinematics and motion control algorithms. To Henk Jan 
Tempert for discussing manufacturing possibilities with suppliers and logistical arrangements. To Tom Wissink and Henri ter Denge for their skills, craftsmanship and entertainment invested to realize the prototype. To Benno Lansdorp for looking for additional clinical applications of the novel system and development of its business case. Above all, I acknowledge Martijn Buijze for his major and indispensable contribution to the development of the mechanical design and realization of the prototype, his open mind to seemingly impossible technical challenges, creativity, knowledge and craftsmanship. As one of the few to fully understand the design philosophy behind the system and the direct link to its success, it is our reluctance to give in to the temptation to cut corners that has lead to a system which is now critically acclaimed and widely celebrated.

In conclusion, I would like to express my sincere gratitude to all involved people, institutes and companies listed hereinafter, for their contribution, major or minor, to the research, development, realization and/or application of the presented system and accomplishment of my mission. Our mission.

Maarten Arnolli,

Enschede, May 2017. 


\title{
DEMCON
}

\author{
Managing Director Dennis Schipper \\ Business Unit Management Michiel Jannink \\ Medical Systems \\ Public Private Partnership Michel Franken \\ Management Tim Gorter \\ Project Management Vincent Heijmer \\ Mechatronic System Engineering Rini Zwikker \\ Peter Rutgers \\ Michel Franken \\ Hernes Jacobs \\ Wouter Hakvoort \\ Benno Aalderink \\ Jos Ansink \\ Marvin Klein \\ Marc van Woensel \\ Alex Rosendaal (intern) \\ Werner van de Sande (intern) \\ Klaas-Jan Gunnink \\ Lennert Molenaar (intern) \\ Mechanical and Industrial Design Martijn Buijze \\ Engineering Lex Lazones \\ Joost Könemann \\ Johnny Tromp (intern) \\ Roy Lubbers (intern) \\ Marco Vernooij \\ Rob van Boom (intern) \\ Otmar Klaas \\ Jan Wiebe Eilander \\ Rob Tuinte \\ Michiel Derksen \\ Henk Jan Tempert \\ Dirk van der Walle \\ Mathijs Panders \\ Douwe Duin \\ Anne de Jager
}


Electrical and Electronic System Michael Harmsen

Engineering Derio Gelink

Jasper Rikhof

Arjan Huisstede

Jorn Zijlstra

Software Engineering Gert Bonestroo

Arjan Flinkers

Gerard Klomphaar

Frank Vlaardingerbroek

Elbert van Beek

Procurement Daniel Reuvekamp

Gül Göksu

Wim Vos

Claudia Kuiper

Niek Aveskamp

Roos Albou

Priscilla Maas

Marleen Breteler

Harmjan Oonk

Logistics Erik Haan

Geri Hulstijn

Prototyping Tom Wissink

Henri ter Denge

Willem Krabbe

Michel Kamphuis

Jelle Mens (intern)

Guus Reith (intern)

Quality Assurance Paul Kosters

Wouter Hagedoorn

Iris van Uitert

Sjoerd Ligthart

Jeroen de Vries

Bianca Screever

Scientific Animation Luke Whitehorn 


\section{Business Development Dirk Binnekamp (consultant) \\ Benno Lansdorp \\ Leon de Jong (intern)}

Public Relations \& Communication Renée Koekkoek op Munsterhuis Marleen Markvoort-Mensink

$$
\begin{array}{ll}
\text { Management Assistance } & \text { Helena Cid } \\
& \text { Judith Scharenborg }
\end{array}
$$

\section{University of Twente}

Precision Engineering Dannis Brouwer

Minimally Invasive Surgery Ivo Broeders

\& Robotics 


\title{
External relations
}

\author{
Massachusetts Institute Nevan Clancy Hanumara \\ of Technology (MIT) \\ Nucletron Jeroen Schuurman \\ (now Elekta) Paul Krechting \\ Johan Henning \\ Paul Visser \\ Philips Healthcare Herman Stegehuis \\ William van der Sterren \\ Quirem Medical Frank Nijsen \\ Radboud University Jelle Barentsz \\ Medical Center Martijn Schouten \\ University Medical Center Koert de Jong \\ Groningen (UMCG) Wim Tukker \\ Wout Heerink \\ Paul Caesar \\ Fokke Boorsma \\ Vincent Ligtenberg \\ Dario Rus \\ Jan Pieter Pennings \\ Simeon Ruiter \\ University Medical Center Wouter Dinkelaar \\ Rotterdam (Erasmus MC) Leo Lampmann \\ University Medical Center Jan Battermann \\ Utrecht (UMCU) Rien Moerland \\ Rogier Schokker \\ Frank Nijsen \\ Rob Bakker \\ Angelique Barten-van Rijbroek
}




\title{
Suppliers of manufactured parts \& services
}

\author{
Ceratec Technical Ceramics Haske van Zadelhoff \\ Futura Composites Marcel de Koning \\ Menno Mathlener \\ GML Instruments Hans Plaggenmars \\ Parts \& Tools Eric-Jan de Jong \\ Pro Air Paragliding Andre Bizot \\ VDM 3D Printing Peter van der Meulen
}
Albert Kip
Arnold \& Siedsma
Becker
Patents \& Trademarks
Watersnijtechniek

beNk

Laserschneidservice

CNC Speedform

Elect

Frejo Machinefabriek

Graveertechniek

Nederland

Hawo

Heijcon 3D Solutions

Hemabo

Horstra Technology

Jan Schartman

Fotografie

Kühne Industrie

LIROS

Luma

Menla

Kunststoftechniek

Odemar Metaal

Papen Metaal Techniek

PPE Almelo

PWB Encoders

R.M. Precision

Strukton Embedded Solutions

Technology Twente

Teijin Aramid

Thales

Veldlaser 


\section{Suppliers of purchase parts}

123inkt.nl

Airbrush Services

Almere

Artaparts

Chain Reaction Cycles

Deli XL

DIT Holland

Elmo Motion Control

Essentra Components

Farnell

G.S.M

Hawo

Hobbyking.com

INDUcoder

Messtechnik

Kinesis

Lapp

Maxon Motor

Motorcycle Products
Acal Bfi

Action

Antratek Electronics

Blokker

Conrad Electronic

Digi-Key Electronics

Edmund Optics

Eltromat

Eurocircuits

Festo

$\mathrm{H}+\mathrm{T}$ Motor Racing

Heijcon

HPC Gears

JAspeed

Koning \& Hartman

Lasaulec

Misumi

Mouser Electronics
Applied Laser

Technology

Brammer Twente

Dealexpress.nl

Distrelec

Elcee Holland

Eriks

Fabory Centre

Nederland

FS Solutions

Habru Aluminium

Lastechniek

Hettykat

I.C.H. Industrial

Components

Jeveka

Kuil Nicos

Lesjöfors Springs \& Pressings

MK Technology

Group

Mouser Electronics 


\begin{tabular}{|c|c|c|}
\hline Multimate & Nanoe & Neita Techniek \\
\hline Ninghai Sendo Sensor & Oliehandel de Croon & Perik Glastechniek \\
\hline Polyestershoppen & PRiTECH & Rexel \\
\hline RS Components & $\begin{array}{l}\text { Schipper Technisch } \\
\text { Handelsburo }\end{array}$ & $\begin{array}{l}\text { Schut Geometrische } \\
\text { Meettechniek }\end{array}$ \\
\hline Skiffy & SMC Pneumatics & Staalmarkt Twente \\
\hline Suter Kunststoffe & Tevema & Thorlabs \\
\hline $\begin{array}{c}\text { Trelleborg Sealing } \\
\text { Solutions }\end{array}$ & $\begin{array}{c}\text { Twimva } \\
\text { Automaterialen }\end{array}$ & United Springs \\
\hline $\begin{array}{l}\text { Vliegerbedrijf De } \\
\text { Paddestoel }\end{array}$ & Xenos & \\
\hline
\end{tabular}




\section{About the author}

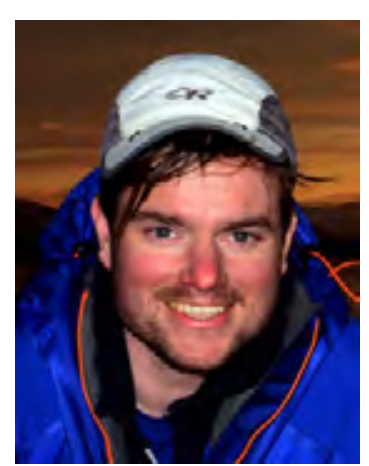

Maarten Arnolli was born on July 13, 1983, in Noordlaren, the Netherlands. He attended secondary education at the Praedinius Gymnasium, Groningen, the Netherlands, from which he graduated in 2001. He attended higher education at the University of Twente, Enschede, the Netherlands, with a major in Mechanical Engineering and minor in International Management, executing a sustainable development project in Peru in 2004 and receiving the Bachelor of Science degree in Mechanical Engineering in 2005. He joined the Laboratory of Mechanical Automation and Mechatronics, conducting research on the rigid and flexible multibody and finite element modelling of the nonlinear dynamics of a flexible parallel guidance in 2008-2009 at Philips Applied Technologies, Eindhoven, the Netherlands, and receiving the Master of Science degree in Mechanical Engineering cum laude in 2009. He joined DEMCON Advanced Mechatronics, Enschede, the Netherlands, in 2011 as a mechatronic system engineer with the privilege to conduct a postgraduate research on image-guided minimally invasive interventions, adjacent to commercial projects in the field of high-tech and medical system development.

Alternating his professional pursuits he embarked on extensive and long term travels in 2006-2007 and 2009-2010 in South America, exploring the Andean mountain range and Guiana Highlands, and crossing the Amazon rainforest. In the period of his postgraduate research he continued trekking, rock- and alpine climbing, canyoning and white water kayaking in the French, Swiss, Austrian, Italian and Slovenian Alps, Scottish Highlands, Spanish Pyrenees and Cantabrian Mountains, Moroccan Atlas Mountains and on Icelandic and Indonesian volcanoes. Larger scale independent expeditions were launched, including a ski traverse of the Greenland ice sheet from Kangerlussuaq to Isortoq (2012), a traverse of the Owen Stanley Range in Papua New Guinea tracing the infamous Kapa Kapa trail (2014), a first attempt in mid winter of a ski traverse of Iceland's Vatnajökull glacier reaching Grímsvötn volcano (2014/2015), and a second attempt completing a full traverse (2017). Closer to home, he resorts to playing piano, sailing, wind- and kitesurfing, hiking, bouldering, ice skating, running, mountain biking and cross country riding on his horse. 

Because the history of evolution is that life escapes all barriers. Life breaks free. Life expands to new territories. Painfully, perhaps even dangerously. But life finds a way.

Michael Crichton 




\section{Development of a precision system for image-guided needle placement \\ - from scratch to clinic -}

Advances in medical technology enable an ongoing transition from invasive, open surgery to minimally invasive diagnostics and intervention. In the field of oncology, percutaneous techniques have emerged in which biopsy and ablation of tumours are performed via a needle through the skin using image-guidance. The objective of this research is the development of a precision system for image-guided percutaneous needle placement in the thorax and abdomen to improve upon the conventional freehand method. It covers the entire strategic, architectural and detail design and realization of a complete system from first idea to prototype, its technical verification, its integration in the intervention suite and its pre-clinical validation, up to its first deployment in clinical practise.

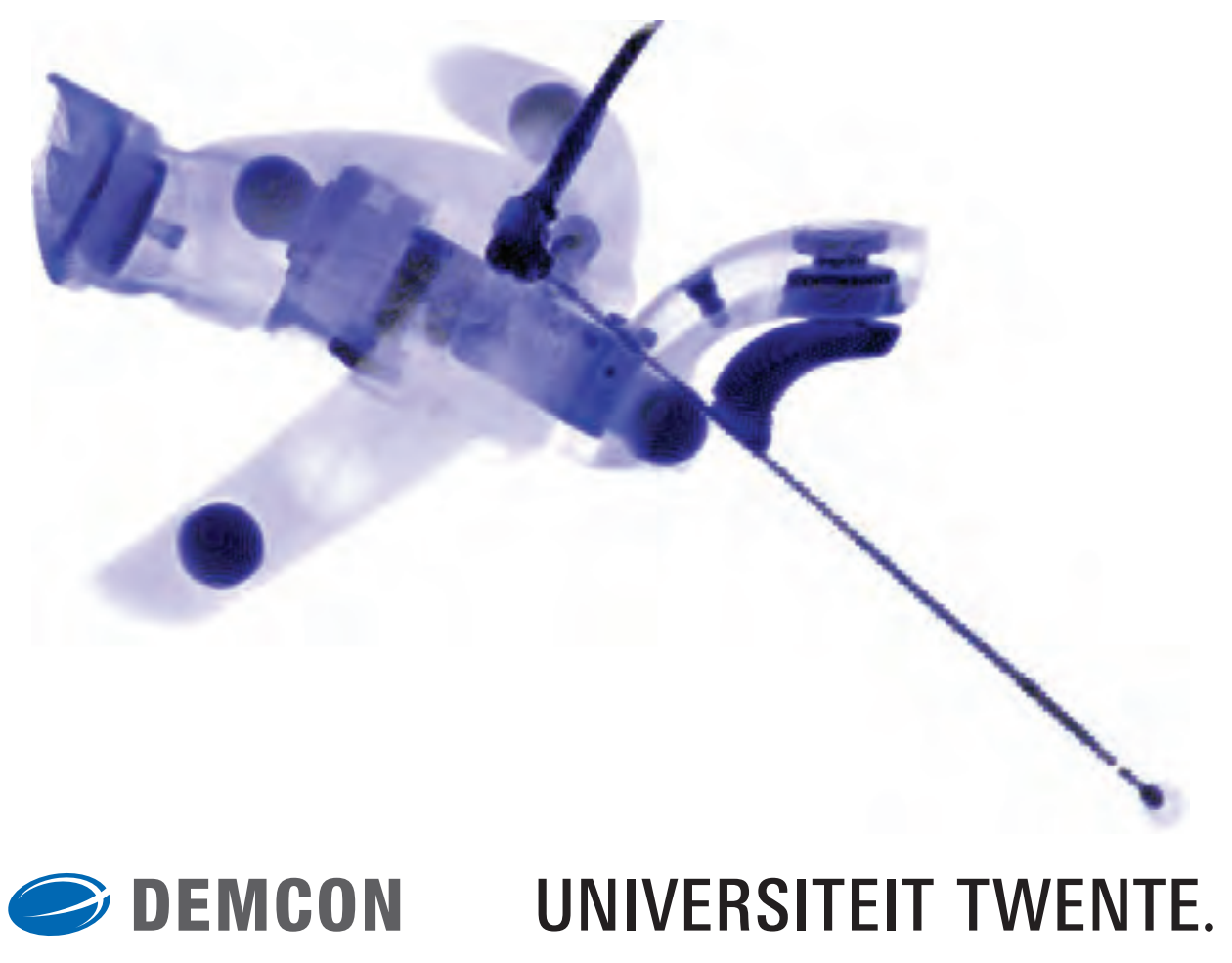

